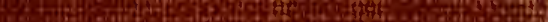

H.

Hog

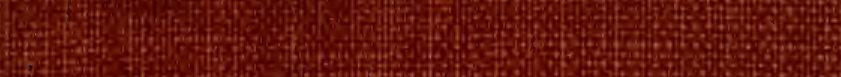

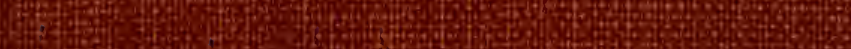

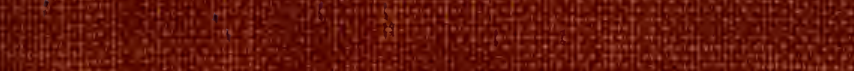

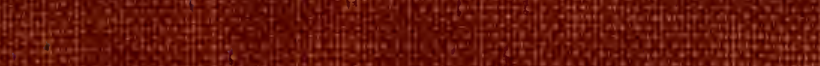

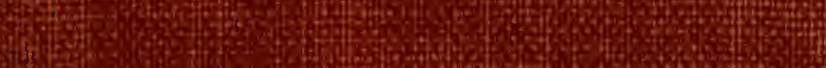

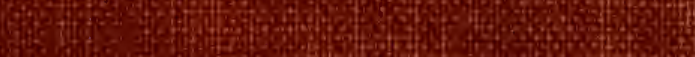
If

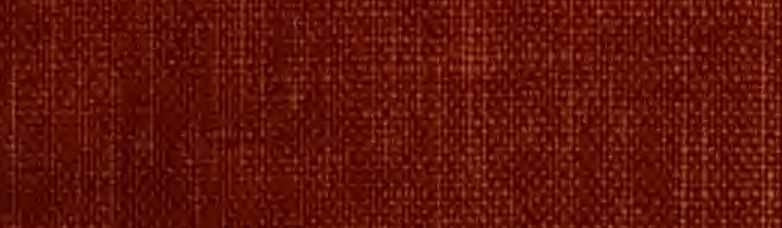
If I0

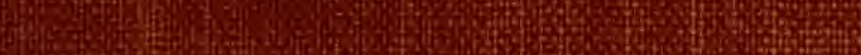
Now

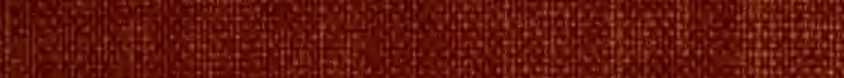

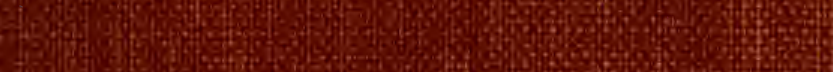
2. (1)

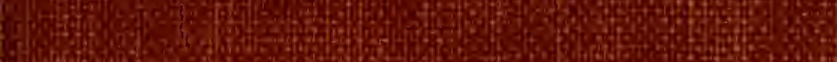

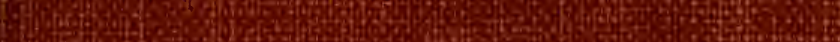
If Wost

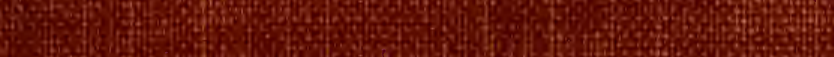

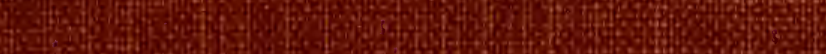

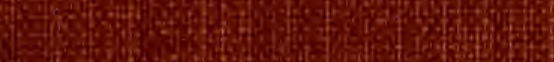
6.

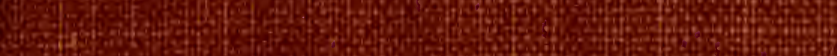
w th

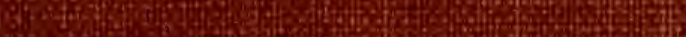
- 


\section{EX LIBRIS}

\section{The Cooper Union}

THE GIFT OF

Érskine Levitt 

GRAMMATICA ARALDICA 


e Corrispondente della Consulta Araldica.

Nuova Edizione con Introduzione ed Aggiunte DI

\section{GOFFREDO DI CROLLALANZA}

Segretario della R. Accademia Araldica Italiana,

Direttore del Giornale Araldico e dell'Annuario della Nobiltà Italiana.

\section{Con 274 incisioni.}

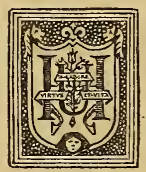

\section{ULRICO HOEPLI}

EDITORE-LIBRAIO DELLA REAL CASA

MILANO

I 904 


\section{PROPRIETÀ LETTERARIA}

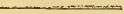




\section{INDエOE}

Pag.

PREFAZIONE

IX

INTRODUZIONE. - Origine e caratteri generali del-

l'Araldica.

Capitolo

I. Definizione dell'arte araldica e classificazione delle armi . . . . . . . . . . . . . 43

II. Scudo e sue varietà . . . . . . . . . . . 47

III. Smalti e tratteggi . . . . . . . . . . . 55

IV. Partizioni e ripartizioni . . . . . . . . 63

V. Figure araldiche. - Pezze onorevoli. . . . . 77

VI. Figure araldiche ordinarie . . . . . . . 99

VII. Convenevoli partizioni . . . . . . . . . 104

VIII. Figure naturali, artificiali e chimeriche. . . 115

IX. Elmo, Cimiero e Svolazzi . . . . . . . . 143

$X$. Delle Corone ed altri contrassegni di dignitù. 151

$X I$. Ornamenti esterni dello scudo . . . . . . 160

XII. Imprese e gridi di guerra. . . . . . . . 164

XIII. Brisure. . . . . . . . . . . . . . . 172

XIV. Leggi araldiche. . . . . . . . . . . . 176

X. Regole generali per blasonare . . . ... 180

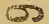

APPENDICE. - Delle livrée . . . . . . . . . . 183 



\section{PREFAZIONE DELL'AUTORE}

\section{ALLA TERZA EDIZIONE}

Questo mio Manuale araldico non è altro che una compilazione e un saggio di studi blasonici.

$D i$ ciò resi avvertiti anche $i$ signori componenti il Consiglio della $R$. Accademia Araldica Italiana nel manoscritto che inviai al concorso, stabilito nell'adunanza del di 8 settembre 1879 ; e che contrassegnai col motto volo non valeo.

Lo stesso ripeto al lettore, ora che questa mia fatica ba l'onore del premio e della stampa.

Il progranma del concorso era espresso con queste parole: " $\dot{E}$ aperto il concorso ad un premio istituito dalla R. Accademia Araldica Italiana per una Grammatica Araldica ad uso degli Italiani, perchè questi possano avere con non molto sludio una sufficiente cognizione della scienza araldica, senza dover ricorrere ad opere voluminose e fattesi rare che trattano diffusamente dell'argomento. "

L'Accademia parvemi avesse a scopo principale una grammatica elementare (della quale manca l'Italia) che risparmiasse a chi avesse avuto vaghezza o bisogno della cognizione di una tanto curiosa quanto trascurata disciplina, la spesa e la fatica di trovare gli antichi trattali della medesina e di perdervi molto tempo a sludiarli. 
Per arrivare a questo scopo, occorrevano semplicità $e$ chiarezza.

Quindi inutile occuparsi della parte storica e degli antiquati quesiti sull'arte medesima; inutile exiandio tutla la parte simbolica che male si accorda colle definizioni precise e colle regole fisse di una scienza, o a parlare propriamente di un'arte della quale si vogliono imparare con poca fatica gli elementi.

Per la stessa ragione conveniva eliminare quanto è di corredo non di necessità all'arte medesima; siccome i particolari riguardanti ai blasoni stranieri; le minute locali differenze del blasone in Italia; le leggende medioevitiche, $i$ tornei, le corti di amore, gli ordini cavallereschi, ecc.

Giovi anche rammentare che $i$ principali scrittori di opere araldiche sono stati per lo passato numerosi e discordi tra loro e nei principii e nei metodi; e quasi tutti fino al Ménéstrier, dominati dai pregiudizii e dalle passioni dei loro tempi, tanto diversi nello indirizzo sociale dai nostri.

Questi riflessi mi pare abbiano guidato $i$ moderni trattattisti dell'arte araldica, tanto Tedeschi che Francesi, $i$ quali banno sfrondato tutto quello che per vecchiezza era rimasto secco e inutil ramo, serbando e coordinando ciò che era necessario alla certezza dell'arte, indispensabile alla archeologia e alla storia.

Presentendo che l'illustre Accademia in mancanza di un lavoro assolutamente perfetto avrebbe preso in considerazione anche quello che contenesse un merito relativo, non credetti presumere troppo del mio presentando questo mio saggio all'illuminato e imparziale giudizio della medesima.

$\mathrm{Mi}$ conveniva essere sobrio nell'abbondanza, chiaro in mezzo a molte oscurità, non affatto barbaro nel tecnicismo, 
che quasi sempre ba faccia di strano; e mi accadde ancora di dovere scartare notizie piacevoli che avrebbero infiorata ma impacciata l'aridità della materia.

Fui pronto anche a correggere quei luoghi che benignamente mi vennero indicati dall'onorevole Commissione esaminatrice.

Questo premelteva nell's I alla mia Grammalica Araldica stampata dall'Accademia: essa ebbe fortunato incontro in Italia e fuori; per cui esaurita in breve l'edizione me ne veniva richiesta una seconda, e quindi una terza che è la presente, nella collezione de' notissimi Manuali Hocpli; ed a questa da me corretta feci qualche opportuna aggiunta.

F. Tribolati. 



\section{INTRODUZIONE}

\section{ORIGINE E CARATTERI GENERALI \\ DELL' ARALDICA. (I)}

L'Araldica ha attraversato tre epoche: nella prima si praticava e non si studiava: nella seconda si praticava e si studiava; nella terza, che è la presente, si studia e non si pratica.

Intendiamoci. Quando dico che non si pratica non voglio negare l'uso ancora sussistente delle armi gentilizie e municipali, il quale è anzi diventato un abuso; nè voglio affermare che tutte le leggi del blasone e tutte le tradizioni araldiche siano state messe in oblio. Dacchè la Nobiltà ha rinunziato ai suoi privilegi, anche le insegne nobiliari hanno perso ogni valore; e se ancora si conservano gli è semplicemente a titolo di ricordi storici o di gloriose memorie; e se ancora si usurpano o si assumono arbitrariamente gli è solo per

(1) Estratto dalla monografia di Goffredo di Crollalanza: Araldica Ufficiale. - Pisa, r89r. 
una malintesa e sciocca vanità. Ma questo uso od abuso oltre a non rispondere affatto all'antico concetto della giurisprudenza araldica, non obbedisce in nulla alle prescrizioni di un'arte dimenticata, di un'arte svisata da quelli istessi che vollero farsene i commentatori, e sulla quale hanno svolazzato per molto tempo i pipistrelli dell' ignoranza.

Dissi che nella prima epoca l'Araldica si praticava e non si studiava. Fu l'età aurea del blasone. Non vi erano legislatori; - dunque non si commettevano delitti. Da ciò si potrebbe inférire che, nella seconda epoca, i delitti suggerirono il codice. Ma per quásto la mia opinione possa sembrare stramba e paradossale, ritengo fermamente che accadde il contrario.

Sul loro nascere le arme erano semplicissime, portate da pochi, consigliate dagli stessi motivi, da una sola necessità, e da gusti presso a poco uniformi. Le divisioni dello scudo, le pezze campeggianti, il disegno più o meno capriccioso delle linee, non significavano nulla, e non avevano altro scopo che di distribuire ed alternare i colori in cento guise per differenziare le insegne. La scelta dei colori stessi non era dettata che da una personale compiacenza per gli uni piuttosto che per gli altri, o dal favore che alcuni di essi godevano già in ciascun paese. Se un simbolismo era loro applicato, poteva dirsi incosciente e spontaneo; anche un bambino trova il nero triste e il rosso giocondo; coll'osservazione della natura gli uomini si sono abituati a considerare il bianco come un segno di purezza e d'innocenza, il verde come un emblema di gioventù e di speranza, l'azzurro come un'immagine di bellezza e di maestà, il rosso come un simbolo del co- 
raggio e dell'amore, il giallo come un attributo di ricchezza e di nobiltà, il nero come un contrassegno di sconforto e di dolore. Se i cavalieri antichi si attennero a questi soli sei colori non fu certo per obbedire a una convenzione intervenuta fra loro, molto meno per evocare il ricordo dei giuochi del Circo, di cui non avevano mai inteso parlare, o per creare dei rapporti misteriosi fra la terra e il cielo, fra le insegne e gli astri; ma presero quelli che erano i più brillanti e $\mathrm{i}$ più appariscenti, quelli che si annunciavano da lontano senza ingenerare confusione, quelli che trovavano ripetuti sotto tutte le forme nella natura stessa, che in ogni tempo avevano figurato negli abbigliamenti, nelle bandiere, nei sacri riti, i colori della gamma trionfante, dinanzi ai quali tutti gli altri si fondono e svaniscono. Le tinte neutre, le gradazioni, le sfumature, i colori misti dovevano essere necessariamente ripudiati; le castellane si spogliavano dei.loro nastri rosa, aurora, foglia morta, dei loro favori violetti, grigi, aranciati, e ne adornavano i loro adoratori; questi si facevano ammazzare per difendere i colori della dama, ma si guardavano bene di smaltarne i loro scudi da guerra. Se alcuno vi fu che a tale uso li fece servire, ben presto si vide il rosa accendersi sino al rosso, l'arancio impallidire sino al giallo, il grigio confondersi coll'argento o decomporsi in bianco e nero; forse il violetto persistette nella porpora, ma questo smalto è araldicamente dubbio, appunto perchè non è che una gradazione del rosso. Nè sorprenda se al bianco e al giallo si.sostitui l'argento e l'oro e se si mise metallo su colore e colore su metallo; le stesse esigenze, che fecero bandire dagli scudi le mezze tinte, consigliarono tale disposi- 
zione, divenuta più tardi uno dei più importanti articoli del codice araldico. Era cura costante dei cavalieri il dare il maggior risalto possibile alle figure dei loro scudi; i più vivaci colori spiccavano fastosamente in un campo d'acciajo, d'argento, o d'oro, e a questi metalli aggiungeva rilievo un fondo scarlatto o un contorno celeste. Pochi sdegnarono o non compresero l'armonia e l'utilità di questa combinazione, ed ebbero il cattivo gusto di caricare argento su oro o d'impiastricciarne verde su azzurro, rosso su nero; le arme loro furono poi dette false ed irregolari se appartenevano ad oscuri gentiluomini, e ad incliesta se ne menavano vanto le più illustri casate; tanto è vero che il solo colpevole è sempre l'asino che mangia un palmo d'erba nel prato altrui.

Nè maggior dottrina e più acutezza di spirito si richiese per la scelta delle figure. Oltre alla croce delle guerre sante ed alle pezze che noi chiamiamo piit o meno onorevoli, e che allora erano onorevoli tutte e a pari grado, poichè tutte brillavano sugli scudi dei cavalieri; oltre a queste pezze che, come dissi, erano pretesto alle svariatissime combinazioni dei colori, e mezzo efficace al differenziare le insegne da guerra, i creatori del blasone ritennero, non inventarono, gli emblemi già universalmente conosciuti prima che l'Araldica li facesse suoi. Erano imagini di corpi naturali, la luna, le stelle, le fiamme, le piante; gli animali da guerra e da caccia, il cavallo, il cane, il cervo, la volpe, il cinghiale; gli animali domestici, il toro, la capra, il gatto; gli uccelli del paese, il falco, la cicogna, il merlo, il corvo, la colomba; gli oggetti che ricordavano il castello feudale, il paese nativo, le abitudini contratte, gli 
esercizi favoriti, i pellegrinaggi, come torri, ruote, freccie, speroni, staffe, anelli, corni da caccia, chiavi, maglietti, conchiglie, ecc. Erano anche simboli popolari, da tutti compresi, motivi ripetuti in mille modi nella ornamentazione, scolpiti nelle satiriche rappresentazioni delle gotiche cattedrali, inalberati come insegne guerresche, ricordati nei proverbi, celebrati nei canti dei trovatori, resi famosi dalle leggende e conservati dalla tradizione: erano il cerchio, il triangolo, il giglio, la rosa, il trifoglio, l'aquila, il cigno, il gallo, il pesce, il leone, il lupo, il serpente, il dragone, il grifo, Melusina, antichissimi simboli, comuni a tutti i popoli di Europa, e che sono forse d'origine ariana, come le fiabe di Cenerentola, di Pollicino e dell'Orco.

Le figure allusive al nome comparvero nello stesso tempo, ed erra chi asserisce che le armi parlanti siano meno nobili e meno antiche delle altre; ne abbiamo esempi che precedono l'uso ereditario degli stemmi.

Tutte queste figure, si vollero, non di colore naturale, ma degli smalti prediletti; altra e più evidente prova di quanto più sopra asserii, cioè che solo i colori araldici potevano rispondere ai bisogni ai quali deve attribuirsi 1'invenzione delle armi gentilizie. Che le figure, e specialmente gli animali, assumessero generalmente, sin dal principio, delle situazioni e delle posizioni speciali, è fuor di dubbio; oltrecchè la forma degli scudi lo esigeva, bisogna anche riflettere che gli artisti di quel tempo erano infelici imitatori del vero e che supplivano spesso con segni di convenzione alla fedeltà del ritratto, come gli antichi scrivevano mulicr formosa, leo ferox sotto le loro dipinture. I quadrupedi furono tutti rampanti e di profilo e quando si volle 
rappresentare un leopardo bastò disegnare un leone passante e colla testa di fronte. Allo stesso scopo si esagerarono i dettagli, le unghie, il becco, la coda, le ciocche di pelo, le penne, le corna, la lingua; le foglie degli alberi, i merli delle torri, i raggi delle stelle, i denti delle ruote ebbero uno sviluppo sproporzionato. Il cane ebbe sempre un collare, il cinghiale delle zanne d'argento, il falcone un cappuccio e dei sonagli, la gru un sassolino in una zampa, lo struzzo un ferro di cavallo nel becco, la colomba un ramo d'olivo, il vepre sette rami e tre radici, ecc. Se le figure erano in numero, se ne mettevano naturalmente più nella parte larga, cioè in capo, che nella parte stretta, cioè in punta. Se alle figure primitive se ne aggiungevano altre più tardi, queste ultime si facevano molto più piccole, anche se nella realtà dovessero rappresentare oggetti più grandi, come ad esempio una rosa accompagnata da tre aquilotti, o un orso circondato da sei torri. Varie pezze geometriche, quali scacchi, plinti, losanghe, bisanti, torte, rappresentavano come potevano le più svariate cose: pietre, assicelle, fusi, cuscini, maglie, monete, pani, frutta, teste di-chiodi, fibbie, cartelli, genme, pezzi dell'armatura, e accessori del vestire, che difficilmente si sarebbero potuii imitare e riprodurre in uno spazio cosi ristretto. Lo stile araldico nacque dalla necessità di accentuare i caratteri delle figure e dalla imperizia degli artisti.

Nessuna legge obbligava i cavalieri a prendersi queste arme piuttosto che quelle, a conservarle od a cambiarle, a modificarle in un senso ovvero in un altro. Ognuno seguiva in tale scelta il proprio capriccio, o il vezzo del tempo. Le brisure non si conoscevano: se 
il figlio portava uno scudo diverso da quello del padre, se i fratelli facevano uso di sigilli con differenti emblemi, non era certo per rispetto alla paterna autorità o per deferenza di cadetti verso il capo della famiglia; gli è che ogni cavaliere voleva farsi distinguere con un' impresa personale, che abbandonava all'occorrenza, quando una conquista, l'acquisto di una nuova signoria, un fatto d'armi, un avvenimento importante, o semplicemente. la mutata fantasia gliene facevano adottare un'altra. Certi signori cambiarono sino a cinque o sei volte il loro sigillo, con figure assolutamente diverse, e nel periodo di pochi anni. Altri invece conservarono sempre le arme originarie e le tramandarono inalterate ai loro figli e discendenti, indistintamente.

Le usurpazioni non erano possibili. Le armi appartenevano esclusivamente ai gentiluomini, e poichè ciascuno di questi aveva le sue, nessuno si curava di assumere quelle degli altri. Ma spesso avveniva che due cavalieri portanti lo stesso scudo s' incontrassero, e siccome nessuno dei due aveva rubato il proprio all'avversario, cosi molto gentilmente e con bella cortesia scendevano in lizza e si battevano senza livore, perchè il vincitore restasse solo a godere dei due pali o dei tre sparvieri contrastati. Quanto al vinto, se non era morto, attendeva una buona occasione per ricaricare di qualche emblema glorioso la sua tavola d'aspettazione. Nè mi si dica che antichissime famiglie portano da tempo immemorabile uno stemma assolutamente identico, e che innumerevoli per esempio sono i leoni d'oro in campo rosso, le croci rosse in campo d'argento, le bande d'azzurro in campo d'oro; osserverete che quelle famiglie appartenevano a paesi diversi, a provincie l'una 
dall'altra lontana, e che non si conoscevano, nè potevano aver rapporti fra loro. Che se ne trovate in uno stesso paese, io affermo le une essere molto più antiche delle altre ed avere adottato uno stemma in epoca più recente.

In queste poche linee si riassume l'Araldica della prima epoca. Lo scudo dei cavalieri la conteneva tutta, ad esclusione delle corone, degli elmi, dei cimieri, dei supporti, delle divise e degli altri ornamenti esterni, ai quali si volle dare più tardi un' importanza tanto capitale quanto ingiustificata. La forma dello scudo stemmato non era e non poteva essere che quella dello scudo da difesa, generalmente triangolare, ma che diventava talvolta quadrato, ovale, rotondo, lunato, incavato; l'insegna araldica si adagiava compiacentemente su tutte le forme; anzi codeste forme erano talmente indipendenti dallo stemma che spessissimo il blasone di un cavaliere occupava tutta la larghezza della bandiera, istoriava la cotta d'arme, e costellava la gualdrappa del cavallo. I più antichi sigilli ci mostrano gli emblemi campeggianti sul fondo, senza scudo. L'arma di una famiglia consiste dunque puramente e semplicemente in una o più figure di un dato smalto sopra un fondo qualunque di smalto diverso. Quanto siamo lontani da certi moderni blasonatori, $i$ quali vorrebbero indurci a credere che la famiglia tale ba per arma uno scudo sannitico o porta una targa accartocciata di.... ecc.! :

Dubito che i cimieri araldici siano nati contemporaneamente ai blasoni. I Crociati non avevano cimieri; $e$ in guerra non si usavano neanche quando i tornei li misero in voga. Ad ogni modo è certo che stemmi 
e cimieri, benchè inventati allo stesso scopo, si mantennero per molto tempo indipendenti gli uni dagli altri; le figure, spesso stranissime, che oscillavano sull'elmo, non erano sempre le stesse che brillavano sullo scudo; passarono poi talvolta dall'elmo allo scudo, e, in epoca meno remota, ritornarono dallo scudo al cimiero. Ma le eccezioni sono tuttora numerosissime. Dopo che le arme divennero ereditarie, i cimieri continuarono a variare; negli antichi sigilli lo stemma di una grande famiglia è sormontato ora da uno, ora da un altro cimiero. Il cimiero non fa dunque parte integrale e necessaria di uno stemma; meno ancora $\mathrm{i}$ supporti che non figurano sui sigilli signorili che come motivi di ornamentazione e non diventarono araldici che in tempi relativamente recenti, rimanendo però sempre arbitrari, come ne fanno fede gli angeli, le aquile, i leoni e i grifi dell'arma dell' ammiraglio di Graville, gli angeli, i leoni, i grifi della casa d'Austria, gli angeli, le vergini, i selvaggi e i leoni della casa di Baviera, e sopratutto i supporti delle arme dei re di Francia che furono ora due leoni, ora due cinghiali, altre volte due dragoni, due aquile, due levrieri, due cigni, due delfini, due cervi volanti, due liocorni, due istrici, due salamandre, due Ercoli, due angeli.

I lambrechini, o svolazzi dell'elmo, erano per lo più ai colori della dama, nelle giostre e nei tornei. Ma non si cominciò a porli nell'arme gentilizie che nel secolo XIV; i nastri che si vedono pendere dagli elmi sui sigilli anteriori a questa epoca sembrano piuttostc correggiole e fermagli da allacciare al mento. Il grido di guerra era molto in uso, non solo nelle feste cavalleresche, ma anche in guerra; negli antichi sigilli però 
non se ne legge sillaba. Quanto alle divise, figuratevi se i Crociati avevano il tempo di lambiccarsi il cervello per stillarne motti arguti e sentenze concettose! Non parlo delle corone di grado, dei mantelli, delle decorazioni e dei contrassegni di dignità, che sono un portato dell'Araldica moderna.

Così riassumendomi, si può affermare che il Blasone, semplicissimo nel suo nascere, circoscritto dal campo dello scudo, tutto compreso negli smalti araldici e in un numero ragionevole di figure geometriche, di simboli popolari e di emblemi conosciuti, affidato alla fantasia dei cavalieri, cui il buon senso pratico e la necessità di evitare ogni confusione valevano di freno, inaccessibile all'ambizione degli ignobili, e protetto dai possessori stessi contro ogni usurpazione, il blasone della prima epoca era scerro d'ogni pecca e non aveva bisogno di codice poichè di per sè stesso faceva legge.

La seconda epoca dell'Araldica comprende due periodi distinti. Durante il primo, gli araldi creano la scienza del Blasone e si studiano in tutti $\mathrm{i}$ modi di renderla misteriosa; nel secondo, gli araldisti creano la Simbolica e si studiano di farla servire alla interpretazione dei misteri inventati dagli araldi. Doppio studio corruttore, di cui vedremo i risultati.

Al primo apparire delle armi gentilizie, gli araldi le considerarono come cosa loro, come un dominio sul quale dovevano avere alta e bassa giustizia. E difatti vi esercitavano tanto la loro azione, che il nome loro ne rimase alla scienza- ed alla pratica del Blasone. In essa introdussero un elemento ottimo: il linguaggio araldico, - un elemento mediocre: la legislazione araldica; - e un elemento pessimo: la cabala araldica. 
Era fra le molte attribuzioni degli araldi l'obbligo di blasonare ad alta voce gli stemmi dei cavalieri che si presentavano ad un torneo. Il bisogno di esprimersi in termini chiari, precisi, rapidi, comprensivi, di evitare la prolissità e le confusioni, e forse anche il desiderio di risparmiare una buona dose di fiato, suggeri a codesti strilloni del medio evo quel linguaggio ammirabile che è il vanto principale del Blasone, e che può considerarsi incontestabilmente superiore a quello di tutte le altre scienze, per eleganza di forme, sonorità di voci, sobrietà di espressioni e precisione tecnica. Gli araldi francesi, che l'usarono pei primi e che ne furono in ogni tempo maestri, seppero risolvere il difficile problema di tradurre fedelmente ed in poche parole lo stemma più complicato, senza perdersi nei fronzuti circuiti e negli scrupolosi meandri delle descrizioni scientifiche.

La corretta concisione della terminologia araldica, ove si sappia maneggiare abilmente, ha per norma precipua di non dire una parola di meno del necessario, nè una sillaba di più del dovere.

Dopo la grammatica araldica, gli araldi misero fuori il codice. Considerando che era loro dovere di impedire le usurpazioni di titoli, di precedenza, di livrea, di bandiera, ecc., stabilirono di loro propria autorità che l'arma pura e piena dovesse appartenere esclusivamente al capo della famiglia, e che tutti gli altri fossero obbligati di alterarla col cambiamento e permutazione degli smalti, col cambiamento, sottrazione o moltiplicazione delle figure, coll'inquartatura dell'arma di feudo o d'alleanza, o coll'addizione di certe pezze, lambelli, bordure, cotisse, bastoni, cantoni, stelle, crescenti, rose, 
gigli, bisanti, anelletti, conchiglie, losanghe, merlotti, ed altri ninnoli di minor conto, il cui sistema di applicazione era bellamente insegnato, principalmente in Inghilterra, mediante una specie di tavola pitagorica. $\mathrm{Ci}$ sarebbe stato invero poco male a differenziare le arme dei cadetti con segni speciali, destinati unicamente a rappresentare le brisure; ma i merlotti, le losanghe, le pezze onorevoli e tutte le altre figure brisanti, non escluso lo stesso lambello, erano già di diritto pubblico nel blasone, e non si poteva indovinare nelle une piuttosto che nelle altre il significato convenzionale che si volle poi attribuire loro; peggio si dica del cambiamento degli smalti o dei mobili, che introduceva la più deplorevole confusione nell'armoriale di uno stato o di una provincia. Il più delle volte l'arma brisata di una famiglia veniva ad essere identica all'arma non brisata di un'altra famiglia; e con questo sistema, per togliere ad un cadetto l'onore di portare 1' insegna legittima della sua casa lo si autorizzava ad assumere quella che non gli spettava, lo stemma di una famiglia colla quale non aveva nessun rapporto. Molte leggi sono fatte cosi: reprimono il reato e fomentano il delitto. La legge delle brisure ingenerò mille disordini nell'organismo araldico, e lasciò a noi, disgraziati araldisti della decadenza, uno stupendo grattacapo. Ma fu almeno osservata, rispettata, codesta legge? No. È fatto assodato che ubbidiva chi voleva. Gli stessi legislatori davano l'esempio della ribellione, ed è rimasto cëlebre quel Roberto d' Andelot, araldo d'armi di Brabante, il quale, avendo osato prendere l'arma piena della casa d'Andelot, si buscò una lavata di capo dalla camera araldica, ed ottenne come 
un favore preziosissimo di poter brisare detta arma con una sbarra di bastardigia, porgendone al capo della famiglia i più umili ringraziamenti.

Gli araldi vollero poi che non si mettesse colore su colore o metallo su metallo, facendo però eccezione per le pelliccie, pel seminato di Francia, per le brisure e per le appendici degli animali; che gli animali fossero messi nella posizione più nobile e rivolti a destra; che il loro piede anteriore destro dovesse andar sempre avanti al sinistro; che fossero dannati al bando del blasone gli asini, i porci, le pecore, le lepri, i conigli, le galline, le oche, ed altre bestiole innocenti, le quali viceversa non si mossero da dove stavano; che il metallo dovesse apparire nel luogo più nobile dello scudo, e che non si ammettessero più di tre smalti, nè meno di due; che le arme non avessero più di tre figure di specie diversa, regola mille volte infranta, specialmente dai Tedeschi e dagli Spagnuoli; che non si introducessero negli scudi figure umane intere, ma solamente mutilate, o le membra sanguinose, e sappiamo che $\mathrm{vi}$ furono anime gentili che si rifiutarono a dare esecuzione al barbaro decreto; che la sbarra fosse riservata ai bastardi, i quali però non ne ebbero il monopolio esclusivo; che si diffamassero gli stemmi dei felloni, degli spergiuri, dei codardi, dei seduttori di vergini, rovesciando i loro emblemi, mozzando la lingua e la coda ai loro leoni, spennacchiando e strappando le unghie alle loro aquile, introducendo dei triangoli ed altre figure geometriche nei loro scudi, e lasciando poi che ciascuno violasse, mentisse, tradisse e gettasse le armi a suo talento, senza togliere una piuma od aggiungere una linea al suo blasone.... 
Non istarò a riassumere tutti gli articoli del codice araldico, nè a rilevarne tutte le infrazioni. Ad ogni peccato, miscricordia. Ma è anche giusto che non si accusi il peccatore dei falli che non ha commessi. Gli araldi medioevali sono assolutamente estranei a tutte le pazze prescrizioni che regolano gli elmi, i lambrechini, le corone, i cimieri, i supporti, i manti e le divise.

Quando l'ordine regnò nel Blasone, — presso a poco come a Varsavia, - gl' instancabili araldi si ricordarono opportunamente che avevano anche missione di far rispettare il nome dei loro signori, e la bandiera e le arme di questi, ecc. E qual più sicuro mezzo per colpire di rispettosa ammirazione e di salutare timore la mente delle plebi che quello di circondare di mistero gli dei inviolabili ed i loro riti? Mistagoghi della nobiltà, epopti dell'araldica, kabiri del simbolo gentilizio, i re d'armi circonfusero di un'aureola nubilosa i parti della loro fantasia, e perchë avessero maggiore autorità li dissero inventati dai pari di Carlomagno, dai paladini della Tavola Rotonda, dai compagni di Ettore e d'Alessandro, dai cavalieri del Santo Graal, e magari rivelati dagli angeli o da Domeneddio in persona. Un sistema completo astrologico-cabalistico-emblematico fu fondato; i pianeti, i segni dello zodiaco, le stagioni, i mesi, i giorni della settimana, le età della vita, le complessioni, i metalli, le gemme, le virtù e i vizi intracciarono una sarabanda sfrenata sui campi armeggiati; gli smalti stessi cambiarono nome; non si disse più gueules, ma carcôme o truty; nè sable, ma sidiros ou perafecy; uno scudo fu fasciato di speranza e di giustizia, o grembiato di smeraldo e di topazio; 
aprile si inchiavò con novembre, il capricorno fu attraversato dalla vergine, e Venere non potè più giacere con Marte senza partorire un' arma falsa e irregolare!

Poi si ripescarono tutte le vecchie leggende e tradizioni druidiche, non ancora spente in quei tempi, e gli stemmi ne furono istoriati. Le fate, i genii, gli elfi, le salamandre, le ondine sbucarono da tutte le parti; i mostri pullularono; quando furono noti a tutti, se ne imaginarono dei nuovi; l'aquila s'innestò al leone, il pesce al cane, il drago al pavone, la capra al gallo; le teste furono raddoppiate; le corna abbellirono la fronte del cavallo, della pantera, della scimia; le ale spuntarono alle spalle dei più pesanti animali; si videro bestie rappresentate in atto di battersi, di leggere, di suonare il corno, di seminar monete, di pascersi dei cibi più eterocliti; orsi mascherati, volpi armate di tutto punto, leoni camuffati da pellegrini, e via dicendo. La vena umoristica e satirica, che serpeggiava dovunque in quell'epoca, esercitava la sua influenza anche sul blasone. Le più strane cose apparvero negli stemmi; e si trovò il modo di rendere enigmatiche anche le figure più semplici e più naturali.

Questa moda piacque alla nobiltà, e la fantasia dei cavalieri si sbizzarri a sua possa. Un immenso punto interrogativo si stese sull'Araldica, e la minima partizione un po' complicata e fuor dell'uso comune divenne un oracolo sibillino. Chi possedeva un blasone singolare ne menava vanto e sfidava il volgo ad interpretarlo; altri giungevano sino a promettere uno scudo d'argento a chiunque sapesse blasonare correttamente uno stemma, come fecero i signori di PressignyMarans. In breve nessuno capi più nulla al simbolismo 
araldico; gli araldi stessi, che avevano spento i lumi, brancolarono nelle tenebre; e da pontefici iniziatori scesero alla condizione di umili valletti del tempio misterioso.

L' influenza degli araldi non si esercitò egualmente in tutti $\mathrm{i}$ paesi, e ve ne furono che seppero sottrarsi alla tirannia di quei pedagoghi dal tabarro armeggiato; cosi nelle repubbliche italiane, ove il blasone, divenuto borghese e popolare quanto all'uso, si conservò quasi sempre puro ed aristocratico nelle sue forme e nel suo stile. Gli stemmi delle antiche famiglie di Venezia, di Verona, di Padova, di Bologna, di Firenze, di Pisa, di Siena, di Genova, ci offrono i più belli esempi di quella semplicità ed eleganza araldica, dalla quale troppo spesso e facilmente si dipartì la nobiltà francese, inglese, tedesca e sopratutto la spagnuola, per dar retta ai fantasiosi consigli dei re d'arme. Se ne togli la Sicilia e le provincie meridionali, ove predominò il gusto spagnuolo, la Lombardia che si risenti dell' influenza tedesca, e il Piemonte che segui tutte le vicende del blasone francese, l'Araldica italiana è forse la più bella, quella che meglio ha saputo custodire le schiette tradizioni dei primi tempi. Fenomeno codesto che dovrebbe sorprendere in un paese ove i comuni schiacciarono di buon'ora la nobiltà feudale, ove le giostre ed i tornei furono feste cittadine più che cavalleresche, ove il capriccio solo dettè leggi agli stemmi, ove il linguaggio araldico si mantenne sempre barbaro, goffo od ampolloso, ove il Blasone -non si cominciò a studiare che in epoca relativamente recente, ove gli araldisti furono scarsi e la maggior parte men che mediocri. Per me, io trovo anzi in questo fatto una prova luminosa di 
quanto asserii, cioè che furono i legislatori dell'arte araldica che provocarono i lamentati disordini, mentre non si può riconoscere loro altro vanto che d'averne insegnato la lingua.

Non dobbiamo poi dimenticare che le nobilitazioni e le concessioni contribuirono non poco ad alterare il carattere primitivo del Blasone. Finchè questo appartenne esclusivamente ai nobili di razza nessun tribunale araldico s'ingeri della scelta degli emblemi; ognuno si componeva un'arma a gusto suo e secondo la moda del tempo. Ma in seguito i sovrani si arrogarono il diritto di conferirne a chi loro piacesse, ed erano naturalmente i loro araldi ufficiali, che sotto il nome di re d'armi, duchi d'armi, marescialli d'armi, giudici d'armi, genealogisti del re, regi armeggiatori, blasonatori di corte, ecc. davano i gigli a piene mani, distribuivano le croci, prodigavano le stelle, affibbiavano losanghe e caprioli, seminavano rose e trifogli, largheggiavano di bisanti e d'anelletti, attribuivano agli uni la gemella potenziata, agli altri la cotissa doppiomerlata, regalavano a destra un'aquila sorante, a sinistra un cinghiale in difesa, e facevano un vero sciupio di castelli, di spade, di mezzelune, di spighe, di colombe, di cuori e di teste umane. Gettandosi a corpo perduto nell'araldica parlante, vollero in ogni cognome trovare una figura, e cacciarono fuori mosche, ragni, formiche, tartarughe, rape, cipolle, meloni, zucche, campane, botti, striglie, fiaschi, soffietti, padelle e mille altri gingilli, di cui s'ingemmarono le armi della nuova nobiltà. Peggio si fu quando la duchessa di Roquelaure suggeri a Luigi XIV d'imporre un balzello sulla vanità umana, e fu causa del famoso decreto del 3 no- 
vembre 1696, in seguito al quale Antonio Vanier, borghese di Parigi, assunse $\dot{a}$ forfait, mediante la somma di sette milioni di lire, la percezione dei diritti che doveano essere pagati per la registrazione di tutte le armi di Francia. Per la tenue somma di 20 lire ogni modesto ciabattino s'ebbe in regalo un magnifico stemma, fatto di metallo o di colore, con due o tre pezze onorevoli e il suo bravo leone rampante attraversante sul tutto. A coloro che volevano restar ciabattini e che si rifiutavano a servirsi di altra arma fuor della loro lesina, i commessi di Vanier, spiritosi come i viaggiatori di commercio, attribuivano d' ufficio scimie, talpe, sanguisughe, pulci, cavolfiori, smoccolatoi, siringhe, scarponi rattoppati e vasi intimi da camera, esigendo pur sempre le solite 20 lire in grazia dell'alto onore conferito. Carlo d'Hozier, Juge d'armes de Sa Majesté et Garde de l'Armorial Général, apponeva placidamente il suo autorevole visto appiè delle facezie araldiche degli agenti dell' imposta.

Intanto il Blasone si studiava con ardore in tutta Europa, e in Francia più che altrove. Gli araldisti, successori degli araldi, pullularono da ogni parte; erano gentiluomini, abati, giureconsulti, tipografi, incisori, eruditi, oziosi, che tutti si proponevano di rimuovere un lembo del velo misterioso che avvolgeva l'araldica classica. La simbolica parve loro la chiave, il sesame apriti del grande arcano. E allora fu visto uno spettacolo grandioso e stupefacente; tutto quanto l'ingegno umano aveva prodottò in prosa o in poesia, da Mosè sino a Rabelais, parve magro pascolo alle fameliche investigazioni di quei dotti ardimentosi. La Bibbia fu messa a ruba, il Talmud divorato, Erodoto fatto a pezzi, 
Omero digerito in men che non si dice. Non trovarono grazia nè l'Apocalisse, nè l'Eneide, nè l'Almagesto. Aristotile e Plinio passarono i più brutti guai; Sant'Agostino fu sacrificato come Petronio; e non scamparono dalla strage nè Diodoro Siculo, nè Strabone, nè Lucrezio, nè Averroès, nè Alberto Magno, nè Agrippa di Nettesheim. Quanto al povero Raimondo Lullo, nulla gli valse di essere stato lapidato a Tunisi; gli araldisti lo fecero a brani senza pietà.

Ma tanto scempio era giustificato. Figuratevi! si trattava nientemeno di dimostrare che il leone è nello stesso tempo il simbolo di Cristo e del diavolo, e significa per conseguenza la virtù e il vizio, la verità e la menzogna; che l'alcione ha la facoltà di sedare col suo canto i flutti procellosi del mare, e rappresenta quindi il savio cittadino che acquieta col buon consiglio i tumulti delle discordie civili; che la lince distingue un oggetto a traverso lo spessore di una montagna, ed è dunque l'emblema della perspicacia; che il leopardo è generato dal pardo e dalla leena, e molto acconciamente si addice ad un bastardo; che il liocorno si addormenta volentieri in grembo alle vergini, e vuol dire continenza e onesto amore; che 1'alloro non è mai colpito dal fulmine, e addimostra intrepidezza; che il carbonchio risplende nelle tenebre di luce propria, ed allude a chiara fama; e che i Battriani si pizzicavano il naso per salutarsi scambievolmente, prova evidente che un naso in uno stemma deve simboleggiare cortesia e buon augurio.

Si trattava anche di ritrovare il significato originario delle pezze onorevoli e di provare che il capo, il palo, la fascia, la banda ricordano rispettivamente l'clmo, la 
lancia, la cintura ed il balteo dei cavalieri; che il capriolo è lo sperone, oppure è un pezzo dello steccato, 0 anche un archipenzolo, a meno che non sia un'armatura da sostenere i tetti delle chiese, il che non impedisce che potrebbe anche essere un cavalletto d'armi destinato a sopportare l'arnese da guerra, se pur non si voglia riconoscervi uno stivale per indicare che il guerriero fu ferito alla gamba; che la croce di Sant'Andrea è lo strumento del martirio di questo apostolo, o una incrociatura di barriera, ovvero una staffa, o come altri vogliono due bastoni stroppicciati insieme per produrre la fiamma, o infine la cifra $\mathrm{X}$ la quale racchiude la perfezione dei numeri; che la pergola, se non è addirittura una forca da impiccare, può essere non solo uno sperone o un pezzo di steccato come il capriolo, ma altresi un sostegno da balestra, un bracciale da sospendervi lampade, un legno forcato da reggere le viti, un pallio da arcivescovo; che il grembo non è altro che un lembo di stoffa tagliato a triangolo, se non si vuole ammettere che sia una controscarpa di bastione, un.gradino da scala a chiocciola, una banderuola da torre, una cornetta di cavalleria, o più semplicemente il grembo di una famiglia da cui uscirono molti uomini di guerra.

Si trattava inoltre di affermare che la losanga rappresenta un ferro di lancia, una pietra, un cuscinetto, una foglia di lauro, un fuso, avvero un'altra cosa che giova tacere; che il lambello figura un collare da torneo, un nodo di nastri, un vezzo di gemme, un tigillo gocciato architettonico, un rastrello da giardiniere, o un ponte; che le otelles dei Comminges possono interpretarsi per ferri di lancia, scheggie di legno, pinocchi 
da tetto, mandorle pelate o piaghe enfiate; e che il vepre dei Créquy è forma alterata di un candelabro a sette bracci, simbolo delle virtù che hanno per oggetto speciale l'onore, o di un ciliegio nano, emblema della benignità di animo gentile.

Si trattava infine e sovrattutto di servir bene chi pagava bene; ragione per la quale ogni gentiluomo di contrabbando si accorse ad un tratto di discendere in dritta linea da Lancellotto del Lago, da Oliviero il Danese, da Carlomagno, da Meroveo, da un console romảno, da un re degli Ircani o da un patriarca amico intimo di Melchissedech; le figure dello stemma chiarivano manifestamente l'autenticità della genealogia. Non parlo dei gentiluomini veri, di cui il blasone sembrò tutta una epopea, i gigli di Francia piovvero dal cielo sul capo di Clodoveo; la croce d'argento dei Montmorency si tinse miracolosamente in rosso a Bouvines; la fata Andaine stampò la sua mano sullo scudo del sire d'Argouges; il gigante Mugello impresse colla sua mazza insanguinata cinque impronte rosse sulla targa d'Everardo de' Medici; Ottone Visconti tolse al gigante Voluce l' impresa del biscione; il goto Alduino, capostipite degli Orsini, che ebbe un'orsa per nutrice, e che mori difendendo il suo stendardo a striscie rosse e bianche, fu sepolto dai suoi soldati sotto uno strato di rose; messer Ubaldino degli Ubaldini conquistò 1a sua testa di cervo sotto gli occhi di Federico Barbarossa, e Federico di Bieberstein il suo corno di buffalo in presenza di Boleslao re di Polonia; gli Asburgo, gli Aragona, i Bourdeille, i Caraffa, i Coucy, conquistarono a prezzo di sangue le loro insegne; molte centinaja di eroi trionfarono di mostruosi dragoni e ne posero la figura nelle loro arme. 
Quando furono illustrati tutti gli stemmi dei vivi, si pensò a gratificarne anche i morti che non ne avevano mai avuto. Gli antichi araldi si erano contentati di attribuirne agli eroi dei tre cicli cavallereschi, gli araldisti ci fecero conoscere quelli della casta Lucrezia, del prode Epaminonda, del cadente Anchise, del rigido Minosse, del cinocefalo Anubis, della sanguinaria Tomiri, del buon Noè che piantò la vigna, del padre Adamo che piantò la razza umana, e di Nostro Signore Gesù Cristo che la redense.

É così che si studiava la scienza araldica nei secoli decimosesto e decimosettimo. Fu il trionfo dell'archeologia delirante.

Ma non bisogna disconoscere che qualche lampo di buon senso illuminò qua e là le opere degli araldisti di quell'epoca, e in ispecie del P. Menestrier, il più ragionevole ed erudito di essi, e di Palliot, il più giudizioso e completo fra quanti scrissero di blasone prima dell'attuale risveglio degli studî araldici. Ottime cose ci lasciarono pure il Vulson, lo Spener, il Cartari, e il Pietrasanta, ma bisogna cercarle pazientemente nel fascio delle solite stravaganze. Che i tratteggi convenzionali per rappresentare gli smalti nei disegni, nelle stampe e nella scultura siano invenzione del Pietrasanta, del Butkens, del Francquart, del Wolfon, o di Filippo de l'Espinoy, poco importa; di questo utilissimo ritrovato siamo in ogni modo debitori ai blasonisti di quel tempo.

E furono gli stessi che intrapresero di regolarizzare gli ornamenti esteriori dello scudo. I re di Francia, lasciando che la borghesia facesse uso di stemmi di famiglia, avevano da molto tempo emanato i più severi 
decreti per impedire l'usurpazione delle corone e degli elmi, distintivi della nobiltà. Ma le infrazioni erano tuttavia frequentissime. Non è dunque da sorprendere se gli araldisti ufficiali ed ufficiosi del seicento si occuparono con tanto fervore della giurisdizione dei timbri, che erano diventati insensibilmente parte importante, se non essenziale dell'arma. - I sigilli del XIII e XIV secolo e le tombe medioevali ci mostrano già gli scudi sormontati da un elmo, attributo principalissimo dei cavalieri; ma quell'elmo aveva la forma in voga e non indicava colla sua posizione o coi suoi ornamenti il grado del possessore dell'arma; più tardi i sovrani, i principi, i duchi, i grandi signori teudali, i cavalieri banderesi l'ornarono spesso di una corona a fioroni o a punte, o di un cerchio gemmato. Il Menestrier pretende che l'invenzione delle affibbiature e delle positure in maestà $O$ in terza rimonti al secolo XIII. Nulla di più falso; gli elmi graticolati apparvero solo verso il $\mathrm{I} 42 \mathrm{O}$, e li portava il conte come il semplice gentiluomo; la distinzione fra la posizione di profilo e in terza è troppo sottile ed anche troppo puerile perchè meriti che ci soffermiamo a discuterla; la posizione in maestà è incontestabilmente meno antica; quanto all'elmo rivolto, se ne vedono numerosi esempi in Germania, e su monete dei duchi di Borgogna, senza che si volesse alludere a bastardigia. Comunque sia, è certo che gli araldisti francesi fissarono definitivamente la materia, la forma e la positura degli elmi, a seconda dei titoli; assegnarono l'elmo d'oro ai sovrani, d'argento ai duchi, marchesi, conti e cavalieri di antica estrazione, d'acciaio ai semplici gentiluomini e ai nobili di recente creazione; vollero che solo gli 
imperatori e i re portassero l'elmo in maestà interamente aperto; che gli altri principi sovrani lo portassero un po' meno aperto (!); che i principi e i duchi non sovrani, i grandi ufficiali della corona, i generali d'armata, i governatori di provincie, ecc.. lo mettessero pure di fronte, ma con nove affibbiature, che vi ponessero sette affibbiature i marchesi; sette egualmente i conti, i visconti e i vidami, ma che il loro elmo fosse situato in terza; in terza quello dei baroni e gentiluomini d'antica estrazione, ma con cinque affibbiature; con tre sole quello dei baroni e gentiluomini, posto di profilo; quasi interamente chiuso (!) quello degli scudieri; chiuso affatto quello dei nuovi nobili; rivolto a sinistra quello dei bastardi. Il più curioso si fu che dopo avere inventato le aperture e le semiaperture degli elmi, quei degni araldisti interpretarono le loro fantastiche distinzioni coll'eterna simbolica, e ci dettero ad intendere che l'elmo aperto in maestà indica suprema chiarezza di sangue; l'elmo chiuso, nobiltà illustre ma senza giurisdizione; l'elmo di profilo, nobilta illustre del cavaliere che dà orecchio ai comandi del suo signore, ecc. ecc. E il più bello si fu il vedere nobili di fresca data, figli di segretari o di tesorieri, discendenti di speziali o di conciatori di pelle, fregiare il loro stemma di un elmo, come se avessero avuto qualche antenato all'assedio di Tolemaide!

L'invenzione piacque in Francia a tutti, tranne forse ai nobili di razza. In Italia la introdussero gli araldi ufficiali, e la misero in credito il Ginanni e i suoi imitatori. Gli Inglesi accettarono per qualche tempo la gerarchia degli elmi, con qualche modificazione nella posizione e nella forma; ma si accorsero ben presto 
dell'assurdità di essa, e soppressero gli elmi interamente. I Tedeschi, più saggi, li conservarono, ma senza alcuna distinzione, poichè fra di loro l' elmo indica la nobiltà e non il titolo.

Meno inconsulta fu la regola che prescrisse di dare ai lambrechini gli smalti dominanti nell'arma. I colori di questa sono per la famiglia che ne fa uso una vera livrea, ed è giusto e ragionevole che figurino anche negli svolazzi dell'elmo.

Ci sarebbe molto a ridire sull' uso delle corone di grado, anch'esse d'invenzione moderna, e le cui forme convenzionali variano a seconda dei paesi. Ma poichè l'applicazione ne è divenuta generale in Europa, e poichè in fin dei conti sono le corone i soli distintivi conosciuti che indichino chiaramente i titoli, lasciamole stare e passiamo la spugna su questo capitolo.

La questione del diritto al cimiero fu appena sollevata da alcuni araldisti. Pietro de Saint-Julien dice che non dovrebbe portarlo se non chi possiede, o è capace di possedere giurisdizione; e il Rocchi pretende che i Conti Palatini e i Cavalieri Aurati, ch'egli chiama dignità imaginaria, possono portar l'elmo, ma non il cimiero, senza particolare concessione; aggiunge che $\mathrm{i}$ magistrati, giureconsulti, dottori, ecc., possono portare un cimiero di qualità competente, come una sfera alata nell'arma dell'astronomo Bianchini. Il Grizio pronuncia che i nobili di recente creazione devono andar circospetti nell'adottarne. Altri più esplicitamente dichiarano che il diritto al cimiero va all'uguale col diritto d'elmo, e che deve vietarsi a chi non puó giustificare l'assunzione del timbro. È questa l'opinione più ragionevole, e se si considera che il cimiero non è che un orna- 
mento accessorio dell' elmo, si potrebbe tutto al più negare che l'elmo stesso ne vada ornato, ma è assolutamente illogico che il cimiero sia conservato all'esclusione dell' elmo (come si pratica dalla nobiltà inglese), o, quel che è peggio, che un cimiero sia rappresentato uscente da una corona di marchese o di barone, collocata sullo scudo, come se ne vedono tanti esempi in Italia ed in Ispagna. I Tedeschi non sono mai caduti in questo errore; essi hanno il torto di attribuire l'elmo anche ai nuovi nobili, ma il cimiero sormonta sempre l'elmo (talvolta coronato), mai la corona sola.

Per me la questione non può esser dubbia; i cimieri ripetono la loro origine dai tornei, ed ivi si portavano sull'clmo; è dunque sull'elmo, introdotto nella composizione dell' arma gentilizia, che devono continuare a collocarsi; il metterli sulla corona fioronata o perlata, innovazione araldica affatto moderna, mi sembra una vera anomalia.

I supporti appariscono nelle armi per la prima volta nel XIV ${ }^{\circ}$ secolo. Pochi signori li usarono costantemente, e siccome non significavano nobiltà antica e cavalleresca come gli elmi, e non rappresentavano un titolo nobiliare come le corone, così non si ebbe occasione di frenarne l'abuso. Gli araldisti tacciono generalmente sul diritto di assumerli. Tutto al più si pretese che solo i sovrani potessero averne due e che $i$ gentiluomini dovevano accontentarsi di uno solo; ma questa regola non fu mai osservata. Il Moreau avanzò senza fondamento che i soli principi della real casa di Francia avessero il diritto di portar degli angeli per tenenti dello stemma; ma nessuno penso a farne rimprovero 
ai duchi di Baviera, ai duchi d'Austria, ai principi di Lippe, ai baroni di Montmorency, ai duchi di Brancas, ai conti di Clancarty, ai conti d'Oxford, e molto meno ai papi. In Francia chi voleva offrirsi il lusso di uno o due supporti non aveva conti da rendere a nessuno; anche in Italia ne prendeva chi ne voleva. In Germania, se si eccettuano le famiglie sovrane, i supporti furono poco ambiti e raramente usati. Vi fu qualche rigore in Inghilterra, perchè ivi i supporti fanno parte essenziale dello stemma e sono occasione a brisura. Gli araldi ufficiali spagnuoli ne limitarono il diritto ai soli Grandi; ma questi il più delle volte non si curarono nemmeno di approfittarne. Nella Svezia appartengono quasi esclusivamente alla nobiltà titolata. In Isvizzera non sono che un motivo di ornamentazione, di diritto comune.

L'origine del mantello proviene indubbiamente dai lambrechini a mantellina e a cappettina, i quali allargandosi ed estendendosi hanno formato una specie di manto scendente sino ai lati dello scudo. I cavalieri lo facevano armeggiato, ma non vi annettevano nessun significato speciale. Nel $\mathrm{XV}^{0}$ secolo questa foggia di lambrechini fu abbandonata e si adottarono quelli frastagliati o a fogliami. I mantelli armeggiati furono ripresi in Francia dai principi e dai duchi verso la metà del $\mathrm{XVI}^{\circ}$ secolo, e si fecero foderati d'armellino, ad imitazione di quelli dei sovrani, i quali erano generalmente rossi o di porpora; nel seicento li vollero anche i pari ecclesiastici, i presidenti del parlamento, i cancellieri di Francia e i marescialli. Il padiglione, che è un mantello sormontato da una cupola o colmo, fu inventato da Filiberto Moreau, ed il re di Francia lo 
portò attorno alle sue arme per la prima volta verso il I680. Quella forma fu ben presto imitata da altri sovrani.

Le divise erano già conosciute prima che gli araldisti le distinguessero in otto classi, ma accompagnavano raramente le armi gentilizie. In Italia la voga se ne diffuse dopo la calata di Carlo VIII; un gran numero di letterati, Monsignor Giovio alla testa, ne fecero soggetto di dissertazioni e di volumi. L' uso se ne generalizzò in breve tempo. Ogni famiglia, ogni città, ogni corporazione adottò un' impresa o una sentenza qualunque. Le accademie ne composero; i parlamenti, le corti di giustizia, i reggimenti, gli ordini religiosi e militari, i capitoli nobili, le società seguirono l'esempio. Sorse allora la grafomane caterva dei simbolisti, degli iconologisti e dei raffazzonatori di imprese, i Bargagli, i Ripa, i Ferro, i Marquale ed altri consimili Picinelli, invidiosi degli allori dell'Alciato, i quali versarono fiumi d'inchiostro per trasformare l'araldica in palestra arcadica; il cervello dei latinisti e dei poeti fu messo alla tortura per stillarne bei concetti e leggiadri motti da farne divise; tutti gli oziosi si consacrarono all'improbo lavoro di cavar sentenze da tutte le rape. Il seicento fu il secolo d'oro dell'araldica ciarliera. Ma la maggior parte di queste imprese erano personali ed apparvero solo in sigilli, decorazioni, marche, etichette, libri, drappi ed altri oggetti, come puro ornamento. Negli stemmi, ove si conservarono quelle ereditarie, frequenti in Italia ed in Spagna, meno comuni in Francia, rare in Germania, di diritto costante nell'alta nobiltà inglese, s'introdussero talvolta anche le divise personali, e si cambiarono a piacere, senza sollevare nessuna protesta da 
parte degli araldisti. Questi riserbarono i loro fulmini per coloro che avessero avuto in animo di arrogarsi il diritto al grido di guerra, proprio delle grandi famiglie feudali e dei cavalieri banderesi; ma nessuno, ch'io mi sappia, pensò neppure a tanto audace usurpazione.

Non rifarò la storia degli ornamenti di dignità ecclesiastiche, della tiara, della mitra, dei cappelli rossi, verdi e neri, dei pastorali e delle croci, che si aggiunsero a poco a poco alle arme dei prelati, perchè questa storia è già stata scritta dalla penna competentissima del dotto Mgr. Barbier de Montault, specialista dell' araldica sacerdotale. $\mathrm{Ma}$ non posso tacere dei contrassegni di dignità militari, giudiziarie e cortigianesche, inventati dagli araldisti ufficiali per rendere più goffi e più pesanti gli stemmi della nobiltà in auge: bastoni da comando, verghe da cerimonia, chiavi da ciambellano, bandiere, cornette, guidoni, spade, cannoni, ancore, mazze d'armi, scuri da littori, scettri di giustizia, corni da caccia, teste di lupo, bottiglie, messali, ed altre pretese marche d'onore, che aggiunte ai trofei di fantasia, ai gran collari degli ordini supremi, ai gran cordoni degli ordini equestri ed ai ciondoli degli ordini minimi, convertirono ogni arma gentilizia in una magnifica mostra di bazar.

La migliore apologia dell' Araldica sarebbe la storia del suo ultimo periodo, vale a dire del suo risveglio e della sua trasformazione nello scorcio del secolo XIX per opera di coraggiosi scrittori e di pazienti spogliatori d'archivii e di biblioteche, i quali non solo dovrebbero lottare colle antipatie dei nuovi tempi, ma anche collo spirito dei loro predecessori, colla vecchia e stagionata tradizione blasonica, colla scienza stessa educata al rigorismo degli araldi e alla pedanteria degli aral- 
disti. Essi dovettero abbattere l'idolo che adoravano per innalzarlo sopra un più alto piedistallo. Oggi l' Araldica ha, fra le scienze ausiliari della storia e fra le arti che formano obbietto degli studî archeologici, il posto che le compete. I dotti le hanno reso la loro stima, e i suoi nemici sono costretti a riconoscere che, malgrado tutti i capi d'accusa formulati contro di essa, non vi è luogo a procedere.

Quel che fosse la nobile scienza delle armi gentilizie sotto il governo dei re d'arme e sotto l'alto protettorato dei blasonatori codicizzanti, l'abbiamo visto. Quel che sia presentemente, sotto la savia direzione di araldisti amici della verità assai più che di Platone, lo dice chiaramente il decalogo della nuova dottrina araldica, il quale sarà forse composto di due o di venti tavole, poco monta, ma alla cui osservanza è ispirato il programma della scuola moderna:

La scienza araldica considerata non piil come la glorificazione di una casta privilegiata, ma come un ramo dell'archeologia e della storia dell'arte e dei costumi;

Il buon senso e la logica sostituiti alla fantasia $c$ al delirio;

La critica vagliatrice dei fatti e delle opinioni;

I monumenti e $i$ documenti autentici soli testimoni riconosciuti degni di fede;

Le induzioni accolte come semplici ausiliari senza che possano essere allegate a titolo di prove concludenti;

Le leggende e le tradizioni rispettate, ma non accettate senza benefizio d'inventario;

I sigilli, le pitture, le sculture, le tappezzerie, le invetriate, le pietre tumulari, tutti $i$ monumenti dell' arte ricercati e fatti oggetto di studio e di pubblicazione, a 
preferenza delle dissertazioni simboliche e dei panegirici genealogici dei vecchi scrittori;

Il simbolismo originario e naturale aminesso in massima, respinto come sistema d'interpretazione;

Le divagazioni astrologiche e cabalistiche lasciate a pascolo dei visionari;

Il blasone mitologico, biblico, eroico, consolare, gotico e carolingio ricacciato nei dominii della favola e dell'epopea;

Gli emblemi dell'aniichità egizia, greca e ronana considerati come geroglifici, come simboli religiosi, come tipi monetari, come contrassegni personali, come insegne da guerra, non come stemmi;

Negata l'autenticità di ogni arma anteriore al mille;

L'origine delle arme gentilizie circoscritta all'epoca delle Crociate e dei tornei;

L'uso frequente delle arme arbitrarie e personali ridotto al XII secolo;

L'uso costante delle arme ereditarie fissato al secolo susseguente;

Le prime regole stabilite dagli araldi ignote sino al principio del $X I V^{\circ}$ secolo;

Le arme, riservate in origine ai soli nobili, in alcuni paesi concesse piu tardi anche ai non nobili o da questi assunte;

Il cimiero, $i$ supporti e la divisa variabili, e ribelli al codice araldico;

La gerarchia delle corone riconosciuta invenzione moderna;

La gerarchia degli elmi, moderna, puerile ed ingiustificabile;

Tutte le figure accessorie dello scudo considerate parti 
non esserziali dello stemma di una famiglia, e la scelta della forma e degli ornamenti esterni dello scudo stesso ridotta a una questione di tatto e di buon gusto;

Distrutto il pregiudizio che le arme parlanti siano meno antiche e meno nobili delle altre;

Sanzionate le leggi araldiche, anche quelle imaginate dagli araldisti, purchè abbiano avuto la consacrazione di una pratica generale e secolare;

Deplorata la regola delle brisure, causa di errori e di confusioni; utili a ricercarsi per l' interesse genealogico, non per ripristinarne il sistema;

Le figure blasoniche studiate nei tipi primitivi e nelle loro alterazioni, considerandone lo stile nelle diverse epoche e nei diversi paesi, e ricostituendolo ecletticamente, per nostro uso, senza allontanarsi dal carattere araldico $e$ senza offendere il gusto artistico e le esigenze del momento;

L'aitico metodo classificativo e nomenclativo ritenuto, malgrado qualche imperfezione, perchè di convenzione universalmente nota;

Le distinzioni sottili, cavillose e pedantesche della veccbia teoria dimenticate;

Il linguaggio araldico, unificato e reso internazionale, giusta le forme proprie di ciascun idioma, sul modello della terminologia e della blasonatura francese, purgato dai barbarismi e dai solecismi, e sfrondato dei pleonasmi e dei sinonimi di lusso;

Le arme borghesi e le imprese municipali, purchè antiche $o$ in altra guisa interessanti, ammesse all'onore degli studi araldici, come le arme della nobiltà;

La pratica tuttora persistente degli stemmi di famiglia, di stato e di comunità insegnata correttamenie agli ar- 
tisti, sottratta all'ignoranza dei possessori, protetta contro le ingiustificate antipatie del volgo, e strappata alle apoteosi impudenti e grottesche.

Gli araldisti dissidenti, gli eretici ostinati negli antichi erramenti, sono ridotti oggidi a una debole frazione nella maggior parte degli Stati d'Europa. Potrei invece citare un centinaio di nomi illustri nella cronistoria del Blasone, se i limiti del mio lavoro me lo consentissero, e se non mi fossi imposta la massima riserva, la quale non $\mathrm{mi}$ permette altre citazioni che quelle che mi sono strettamente necessarie.

La Germania e l'Austria raccolgono il gruppo più numeroso degli araldisti ortodossi. Berlino e Vienna, sedi delle benemerite Accademie Herold e Adler, sono i due centri più importanti del movimento araldico della nuova scuola. Chi non ha avuto occasione di ammirare i capolavori di pazienza, di erudizione e di squisito gusto artistico, pubblicati da quei valenti ricercatori di vecchie cose, non può farsi un concetto esatto dei progressi della scienza araldica. L' Olanda e il Belgio rivaleggiano, benchè in proporzioni più modeste, coi due imperi tedeschi. La Francia avrebbe il primato su tutte le altre nazioni, se non vi pullulassero ancora gli araldisti eterodossi, i genealogisti mercenarii e le agenzie nobiliari. I Francesi conservano però sempre il vanto di eccellenti blasonatori; il linguaggio araldico è da essi inteso ed adoperato anche da quelli scrittori che non si occupano d'araldica, e non mi è mai accaduto di leggere una descrizione barocca o incomprensibile di uno stemma nelle pagine di un libro di storia o d'archeologia, e nemmeno in un articolo di gazzetta. La Francia è inoltre la terra clas- 
sica delle grammatiche e dei dizionarî araldici, degli armoriali e dei nobiliari. Lo stile blasonico, che da quasi due secoli vi aveva assunto una maniera goffa per un riprovevole riavvicinamento all'imitazione della natura, si è ingentilito da qualche tempo in qua ed ha ripreso il carattere tradizionale.

La Svizzera ci fornisce, col suo amore per i monumenti e per gli studî araldici, la migliore confutazione del pregiudizio invalso fra noi, essere cioè il blasone un passatempo da aristocratici e da codini. Gli araldisti svizzeri sono quasi tutti educati alla buona scuola; i loro pittori e incisori vanno di paro con quelli della Germania. L'Inghilterra subisce ancora l'influenza dei suoi araldi ufficiali; ciò non pertanto non ha penuria di araldisti ragionevali; e lo stesso dicasi della Svezia, della Danimarca e della Russia. Ultima viene la Spagna, ove, se ne togli il Fernandez de. Bethencourt che ha infuso un po' di sangue giovane nelle vene della decrepita scienza d'Argote de Mòlina, tutti i genealogisti continuano a scoprire nuove ed ascose virtù nei simboli eroici dei troppo numerosi discendenti dei re Goti.

E l'Italia?

Ah! è giusto; occupiamoci un po' anche del nostro paese.

L'Italia, patria dell' erudizione archeologica, focolare delle arti belle, tempio delle tradizioni gloriose, e, nello stesso tempo, covo di molti pregiudizi popolari e di molti astii preconcetti, non si è nemmeno accorta dell'araldica metempsicosi. Per essa il Blasone è sempre la scienza vana, assurda, spregevole, turiferaria dell' $\mathrm{O}$ limpo nobiliare. É fra noi che l'Araldica è veramente impopolare, è fra noi che non ha saputo conquistarsi 
nè il rispetto dei cittadini, nè la stima dei dòtti, nè il culto dei patrizi. È fra noi che il seme delle nuòve teorie ha fruttato più lollio che frumento; è fra noi che il collegio degli iniziati d'Iside è sopraffatto dalla legione dei violatori del tempio; è fra noi che un araldista coscienzioso è condannato a fare in un circolo di letterati la figura del corvo rivestito delle penne del pavone.

Eppure anche l'Italia ha cooperato al risveglio ed alla riabilitazione degli studî araldici. Sin dal i875 la Regia Accademia Araldica Italiana, residente in Pisa, ha riunito una eletta e numerosa schiera di collaboratori onesti ed operosi, ai quali furono aperte le colonne del Giornale Araldico-Genealogico-Diplomatico, che conta al presente il suo ventottesimo anno di vita.

Anche fuori del seno dell'Accademia non pochi scrittori di materia blasonica acquistarono una certa riputazione. Ma diciamolo subito: in Italia vi è copia di eruditi, di dilettanti, di persone più o meno competenti in fatto di stemmi; ma $\mathrm{i}$ veri araldisti sono pochi, anzi pochissimi; ed anche fra questi, i dogmi del famoso decalogo araldico non hanno ancora ottenuto la piena credenza che meritano. Quanto agli araldisti dissidenti ne troviamo d'ogni fatta: di quelli che non giurano che sulla fede di Mgr. della Chiesa, e di quelli che non riconoscono autorità alcuna al di sopra del $\mathrm{Mu}-$ gnos; di quelli che la pensano come il Capaccio, e di quelli che dividono le idee del Campanile; di quelli che romperebbero una lancia in onore del Ginanni, e di quelli che accetterebbero la prova del fuoco per la difesa del Beatiano. Molti ci parlano ancora degli stemmi dei legionarî romani, o del giglio donato da 
Carlomagno alla città di Firenze; altri interpretano araldicalmente le dodici gemme dell'ephod e le insegne dei sette eroi di Tebe; o vaneggiano della simbolica del rosso e dell'origine misteriosa dell' aquila bicipite; i più discreti si ostinano a cercare Amedeo $V$ fra $\mathrm{i}$ difensori di Rodi. Non parlo di certi genealogisti di mia conoscenza, pei quali il blasone non è che un pretesto a giustificare la discendenza di una famiglia dai Vituli, dai Lentuli, dagli Annii, dal re Poro o dalla regina di Saba. Tutti poi sono d'accordo per storpiare il linguaggio araldico in modo che se gli ignoranti di blasone non ci capiscono meglio di prima, gli araldisti si dannerebbero volentieri per intenderne una sola sillaba.

L'antipatia per la scienza araldica è così prepotente nell'animo dei nostri letterati e dei nostri storici, che non uno di essi saprebbe blasonare correttamente lo stemma della città di cui ci narra le vicende o di cui ci illustra i monumenti. "Il n'est guère admissible scrive il conte A. de Foras - qu'un lettré, un homme du monde, n'aient une teinture du Blason, comme de toute autre science. » Quanto costa per ottenere quella debole tintura araldica che basti ad uno scrittore per rendere intelligibili le sue descrizioni e per salvarsi dai motteggi della critica? Pochi giorni consacrati alla lettura e allo studio di un libriccino elementare, la Grammatica Araldica del Tribolati, e la consultazione di un dizionarietto tecnico, il Vocabolario Araldico del Guelfi, che si trovano dappertutto, poichè fanno parte della collezione dei Manuali Hoepli. Ma no! I nostri scrittori preferiscono confondere il partito collo spaccato, la banda colla sbarra, l'orlo con la bordura, e perdersi 
in circonlocuzioni grottesche quando hanno esaurito il loro repertorio di termini pretesi tecnici, piuttosto che riconoscere all'araldica un granello di utilità. Ed esigono che i poveri lettori comprendano qualche cosa o trovino il modo di farsi spiegare da qualcuno descrizioni su per giù di questo genere: Una sbarra traversa diagonale, ornata di quadretti all'ingiro e di tre pallottole rosse nel mezzo, con un leone rampicante, di color bruno, mezzo nascosto dalla sbarra, che guarda fisso una stella d'oro in alto, e colle zampe poggianti su di un prato; il campo superiore turclino, e l'inferiore bianco. Oppure: Due filari di piccioni verdi che s'incrociano a sgbembo con una sottile fascia nera; tra $i$ picconi una serpe strisciante; il campo di cielo caricato di uccelletti, con nicchio in un angolo. $\mathrm{O}$ anche: Lo stemma è di campo dimezzato rosso e bianco: la parte rossa è suddivisa in due zone gialle; sul bianco v'è un gallo nell'atto di cantare, stante sopra una collinetta verdeggiante che è circondata da un fiume; e in lontananza si vede una rocia oggi distrutta. Vorrei che l'egregio autore della Guida. storica di un'antica città dell'Umbria mi spiegasse cosa intende per circolo d'oro listato in rosso con un emblema nel centro in campo rosso, per variato in croce d'azzurro e d'oro, per quattro fiamme pendenti da una banda, per campo color verderame traversato obliquamente da una fascia, e per cento altre perle del più puro oriente che ingemmano la sua dizione araldica. E che dire di un dotto archivista degli Abruzzi, il quale nel descrivere gli stemmi dei comuni della provincia, si crede obbligato a spiegarci come ogni comune abbia il suo stemma "che altro non è se non la precisione della sua terminologia » come l'uso ne sia antichissimo e rimonti a 
Giuda figlio di Giacobbe, mentre però « nella nostra Italia l'uso di esso non è anteriore che al decimo secolo », come " il campo divides'in quello di partito, di spaccato, di tagliato e di trinciato, secondo le diverse linee o fasce orizzontali e diagonali », e come « il Douët D'Areg (leggi: d'Arcq) assegna il linguaggio degli stemmi che li chiama tipi e li distingue in otto classi », le quali viceversa non sono che le otto classi dei sigilli ? E poichè questo brav'uomo d'archivista provinciale ha studiato con tanto zelo la storia degli stemmi, non poteva egli dare un'occhiatina anche alla grammatica araldica, tanto da risparmiarsi i 467 spropositi, che ho avuto il dolore di rilevare nei 285 stemmi da lui blasonati? Non giova asserire che « $\mathrm{i}$ dotti hanno oramai constituito questo studio in dottrina propria con i nomi di sigillografia e stemmografia, sicchè i paleografi e i diplomatisti, sebbene sieno stati i primi a prendere in esame questi monumenti di antichità, debbono tenerli come parte accessoria del loro soggetto principale »; occorre anche mettere i disgraziati lettori in grado di veder chiaro in un ovato nero con un mezzo cerchio rovesciato con sopra due uncini, e insegnar loro il modo di disegnare uno scudo tutto di fasce triangolari alternate di oro, di argento e d'azzurro!

L'ignoranza di cose araldiche passa in Italia ogni confine. Cento volte mi è accaduto di dover raddrizzare le più storte opinioni e le più strambe idee, espresse con molto convincimento da egregi amici, da persone ammodo ed istruite. Gli uni mi mostravano il loro stemma figurante una fascia increspata o due leoni affrontati, e pretendevano ad ogni costo che sulla base di simili documenti io narrassi loro le origini e le vi- 
cende della loro famiglia. Gli altri mi pregavano al contrario di comporre «il loro blasone », aggiungendo che $i$ loro antenati erano venuti dall' Albania e che parecchi erano stati guerrieri di valore. Non pochi sostenevano energicamente che lo stemma della Francia ¿̀ un gallo, ¿quello della Russia un orso, quello della Svizzera il cappello di Guglielmo Tell; che tutti coloro che hanno una croce nell'arma discendono da crociati; che l'elmo araldico si chiama cimiero, e che esso cimiero deve essere di ferro ed ornato di piume; che $i$ marchesi portano la corona con cinque punte, $\mathrm{i}$ conti con sette palline, $\mathrm{i}$ baroni con tre foglie di acanto, mentre i nobili non titolati hanno la corona con cinque fioroni! Vi fu persino chi si ostinò a persuadermi che il suo stemma era un cane barbone bianco, tosato a metà, seduto sopra un tavolino di legno, in un campo color mattone! Il curioso si è che raramente mi riusci di convincere codesti signori dei loro errori; il più delle volte chi ci passava da ignorante ero proprio io.

Ovunque, all'estero, una famiglia nobile conosce perfettamente il proprio stemma, e si guarderebbe bene dall'alterarne uno smaito, dal cambiarvi la posizione di una figura, dal toccarvi una sola linea. In Italia non è cosi. Il capriccio vi detta leggi, come nel primo periodo dell'araldica. Le fascie si fanno dritte o centrate, le bande ondate o increspate, $i$ leoni rivolti a destra o a manca, gli alberi terrazzati o sradicati, le stelle a cinque o a nove raggi, le rose con gambo o senza, le figure umane vestite alla romana, alla moresca, o nude, a piacere; non si fa distinzione alcuna fra tre pali e un palato; la croce piena diventa scorciata colla massima disinvoltura; le bordure si aggiungono o si tolgono 
con molta indifferenza; dove avete visto oggi un leone, trovate domani un leopardo; se un delfino campeggia libero nel centro di uno scudo, non è improbabile che si tuffi nell'acqua alla prima occasione; che vi siano cinque fusi o sette losanghe in uno stemma, poco monta, purchè ognuno sia libero di cambiare un grifo rampante in un dragone passante. Non c'è poi gran male a far uscire un elmo da una corona, o a far questa perlata anzichè fioronata! E che torto si può fare all'Araldica se uno spaccato d'argento e di nero s'è trasformato in uno spaccato di nero e d'argento, se invece di un'aquila semplice si assume un'aquila bicipite, e se si tolgono la sella e le redini ad un cavallo inalberato per lasciarlo galoppare allegramente sopra una campagna cosparsa di fiori ?

Questi e simili arbitrii araldici sono meno rari di quanto si possa credere. Io non posso gettar gli occhi sopra una carta da visita stemmata senza reprimere un moto di compassione. Ho visto stemmi scolpiti, dipinti, smaltati, ricamati, in case patrizie, che di tutto sapevano fuorchè di blasone. Che disegno, che stile, quali figure, quali colori, eterni Dei! Che i nobili siano i primi ad adulterare le loro arme gentilizie, è strano e lamentevole, ma ci devono pensar loro; che gl'Italiani abbiano della scienza araldica la stessa conoscenza che ho io dell'arte di imbalsamare $\mathrm{i}$ morti, passi ancora; ma che in Italia sia depravato a tal segno il gusto, il sentimento artistico, non lo posso nè ammettere, nè concepire.

Guai se scendiamo ad esaminare le facezie dell'araldica industriale! Come i cappellai, i liquoristi, i farmacisti, i profumieri, i fabbricanti d'ogni genere ci hanno 
conciato le insegne reali d'Inghilterra, di Spagna, di Portogallo, di Grecia, d'Italia! Che bella mostra fanno sulle réclames illustrate, sui manifesti di esposizioni, di corse, di feste, sui diplomi d'onore, su molte medaglie, ecc., i nobili stemmi di Roma, di Torino, di Venezia, di Bologna, di Palermo, di qualunque delle cento città, insanamente alterati, falsati, disegnati a controverso, coloriti di tinte indescrivibili, ridotti a figurine burlesche, a pupazzetti degni della matita di un caricaturista!

Se all'estero l'araldica industriale pecca spesso contro le regole, non giunge però mai ad oltraggiare i caratteri del Blasone.

Una serie sterminata di sconcezze, di caricature e di mostruosità araldiche ce la offrono gli stemmi delle piccole città e dei comuni d'Italia. Rari sono quelli che si lasciano blasonare con esattezza, e resta ancora il dubbio che siano i veri ed autentici. Nessuna nozione di smalti; ignoranza dei tratteggi; numerose le varianti delle figure; eteroclite le posizioni di queste; inconcepibili gli attributi delle pezze; indecifrabili gli accessorî e i mobili di secondo ordine; inenarrabili i paesaggi e le scene che ritraggono. $\mathrm{E}$ il caos fatto blasone! La fantasia ha immaginato mille forme di scudi stravaganti, gli ornamenti meno attendibili, le divise più stupefacenti. E le corone? Murali, ossidionali, vallari, castrensi, principesche, comitali, baronali, e la corona di Carlo V, e la corona di Napoleone I, e la corona di Davide, e la corona del Prete Janni, e la corona del re di coppe; non v'è tipo, reale o fantastico, che manchi al campionario. Se vi rivolgete agli illustrissimi sindaci dei rispettivi comuni per avere schiarimenti, ne avrete risposta, quando si degnano di rispondervi, in questi termini: 
Lo stemma ba lo scudo in argento con color rosso ovale alla italiana incartucciato diviso in quattro (testuale!), e vi converrà fare un patto col diavolo per capire che si tratta semplicemente d'un inquartato di rosso e d'argento. Se poi vi rivolgete all'archeologo della località, vi manderà un trattato di araldica cabalistica o una dissertazione sui viaggi d'Antenore, proprio per farvi perdere quel po' di testa che vi rimane.

Potrei citare esempi a centinaja e pubblicare documenti autentici di quanto espongo. Ma parmi averne detto anche troppo. Alla prossima festa nazionale divertitevi a guardare le bandiere italiane; vi do' parola che ne troverete, fra cento, per lo meno una coi tre colori perpendicolari all'asta, un'altra col verde al posto del rosso, una terza col verde sostituito dal turchino, una trentina senza stemma reale, una dozzina collo stemma incoronato e cinto da bordura azzurra, altrettante colla semplice croce bianca in campo rosso, e il resto colla crocetta della Convenzione di Ginevra: rossa sulla zona bianca!

Quando si ignora come è fatta e come deve essere la bandiera del proprio paese, non c'è più da meravigliarsi se si dicono e se si praticano tante panzane nell'araldica privata. A chi vuole evitarle consiglio la lettura delle regole esposte con tanta semplicità e con tanta chiarezza pratica dal compianto mio amico e collega Felice Tribolati, di cui assunsi di rivedere ed ampliare la presente nuova edizione per invito del benemerito editore comm. Ulrico Hoepli e previa autorizzazione della famiglia dell'autore.

G. Di Crollalanza. 


\section{CAPITOLO I.}

\section{DEFINIZIONE DELL' ARTE ARALDICA}

E CLASSIFICAZIONE DELLE ARMI.

Dicesi arma o stemma il complesso di tutte le figure, emblemi, smalti, ornamenti, contrassegni d'onore, che servono a far conoscere la nobiltà di una famiglia, o a distinguere una nazione, una provincia, una città, una corporazione, o anche una famiglia non nobile.

Il nome arma ebbe origine dalle armature su cui anticamente si raffiguravano questi emblemi gentilizi. La voce stemma viene dal greco; өгвu. гтx erano quelle tessere su cui erano dipinti i ritratti e segnati i nomi degli antenati e i gradi delle discendenze.

Le opinioni dei dotti sull'antichità dell'armi o insegne gentilizie sono molte e svariate; non le riferiremo, non essendo nostro compito di rifarne la storia. Diremo soltanto che le armi presero l'idea dagli antichi emblemi personali, cominciarono a prodursi nei tornei ed ebbero maggiore sviluppo dalle Crociate, perfezionandosi poi per opera degli araldi, che stabilirono regole costanti a partire dal XIV secolo.

L'araldica è dunque l'arte degli araldi, l'arte che 
insegna a comporre le insegne gentilizie. L'araldica inventa le bizzarre divisioni dello scudo, crea imagini fantastiche, ricerca nella mitologia, nella storia, nell'archeologia, nel costume dei popoli per trarne figure allusive a nomi e ad avvenimenti, e traccia con segni emblematici sugli scudi delle famiglie, delle città e delle nazioni le vicende, le appellazioni, i titoli e le particolarità di esse, servendosi di un mezzo conosciuto da tutti i popoli, il simbolo.

Dopo l'invenzione dell'armi gentilizie, gli araldi o re d'armi ebbero ufficio di conoscerne tutte le usanze e tutte le leggi che le regolavano, studiare i diritti della nobiltà, impedire le usurpazioni, conservare i registri genealogici e gli armoriali, e gridare la descrizione delle insegne di quei cavalieri che, presentandosi a combattere in un torneo, suonavano il corno per chiamare gli araldi stessi, onde venissero a riconoscere il loro stemma. Dal tedesco blasen (suonare il corno) provenne la roce blasone. Questa etimologia, che è la più generalmente adottata dagli araldisti, non è peraltro accertata; altre ne furono proposte, ma tutte egualmente dubbie.

Il blasone è la scienza che insegna a decifrare le armi, a comprenderne il significato nelle diverse figure, le proprietà, le leggi dell'araldica, e a descrivere in linguaggio tecnico qualunque specie di insegne. Malgrado questa distinzione, le voci araldica e blasone sono in gènerale prese l'una per l'altra.

Blasone fu anche detto impropriamente e poeticamente per arma, come: $i$ blasoni degli antenati, polveroso blasone, il blasone dei re di Francia...., ed anche per nobiltà, come: il blasone non fa la virtil.... 
Diverse sono le divisioni che gli araldisti hanno voluto fare delle armi o insegne gentilizie.

Il Bombaci (nel suo Araldo) divide le armi in tre specie: il Cellonese (nel suo Specchio simbolico) le divide in cinque specie diverse; ma i più autorevoli, e anche i più moderni, si attennero alla divisione del celebre Ménestrier nel suo Compendio dell'arte araldica.

Egli divide le armi in sei principali specie, e sono:

I. arme di dominio, come quelle di tutti i regni e principati e repubbliche sovrane;

2. di dignità, ossia contrassegni della carica;

$3 .^{\circ}$ di comunità, come quelle delle provincie, città, religioni ed altre simili università;

4. ${ }^{\circ}$ di concessione, quelle che si concedono dalle potestà sovrane, prese dalle armi loro medesime;

5. di padronanza, cioè quelle che si aggiungono alle proprie per contrassegno di dipendenza;

6. gentilizie, che sono le armi proprie delle famiglie e colle quali si distinguono l'una dall'altra.

Altri aggiungono altre cinque specie, e sono:

I. armi di alleanza, contenenti i quarti degli avoli;

2. ${ }^{\circ}$ di successione, vale a dire quelle di cui uno si serve in mancanza dell'erede del sangue;

3. di sostituzione, cioè quando uno è obbligato ad assumere il nome e l'arma di una famiglia estinta;

$4 .^{\circ}$ di assunzione, l'arme che si aggiunge alla propria in memoria di qualche gloriosa azione;

5. di pretensione, l'arme di feudo o dominio sul quale si pretende qualche diritto e che s'inquarta collo stemma di famiglia.

Del resto queste divisioni non sono rigorosamente scientifiche, e a seconda del sistema adottato nello stu- 
dio dell'arte araldica, può farsi a talento questa divisione.

Non è inutile conoscere anche il significato delle seguenti denominazioni:

Armi semplici, ove non si riconosce che uno stemma solo non inquartato.

Armi composte, che si formano di due o più armi inquartate o altrimenti riunite in un solo scudo.

Armi parlanti o agalmoniche, in cui le figure alludono al nome della famiglia che le spiega.

Armi simboliche, che alludono a qualche fatto tradizionale o storico, od anche ad alcuna invenzione leggendaria.

Armi dimandanti o d'inchiesta quelle che, essendo composte contro le regole del blasone e pure appartenendo ad illustri casate, danno motivo di ricercare perchè siano di tal fatta, a ricordo di qualche azione gloriosa.

Armi false o irregolari quelle che sono contrarie alle regole del blasone, senza motivo di inchiesta.

Armi pure e piene quelle che rappresentano lo stemma della famiglia senza alterazione di sorta, cioè senza brisure, nè inquartamenti, nè concessioni, ecc.

Armi spezzate o brisate quelle in cui fu introdotta qualche brisura o spezzatura per riconoscere le linee ultrogenite, oppure i bastardi.

Armi diffamate quelle in cui fu introdotta qualche figura disonorante per togliere il pregio dell'arma in seguito ad azioni condannate dalle leggi cavalleresche. È dubbio che simili armi siano mai state in uso, malgrado i pochi esempi storici che se ne danno.

Sul significato delle armi nazionali, municipali, personali, ereditarie, abaviche, materne, muliebri, legittime, arbitrarie, ecc., non giova soffermarsi. 


\section{CAPITOLO II.}

\section{SCUdo E SUE VARIETÀ.}

Lo scudo senza dubbio forma una parte integrante dell'arme, cioè dell'insegna, ed c̀ il fondo o il campo sul quale sono figurate le armi.

Si sa che lo scudo era un arnese difensivo di guerra delle milizie dell'antichità e del medioevo, e dismesso soltanto dopo l'invenzione e il perfezionamento delle armi da fuoco: in uso ancora soltanto presso alcuni popoli selvaggi.

Nel significato araldico, lo scudo è quella figura destinata a ricevere gli smalti, i colori le partizioni, gli emblemi di un'arme gentilizia od insegna blasonica.

La forma dello scudo cambia secondo le diverse epoche nelle quali fu usato.

Gli antichi blasonisti enumerano le svariatissime forme che ebbe lo scudo militare appo gli antichi popoli, e specialmente quello dei Greci e dei Romani.

Per noi sarà più utile accennare le principali sue forme nel medioevo fino all'età presente.

Nel XII secolo e nella prima metà del secolo XIII, 
lo scudo era alto la metà di un uomo, triangolare, nella parte inferiore appuntato e in quella superiore rotondato; di guisa che riparava mezza la persona del guerriero, e veniva portato attorno alla spalla mediante un cintolo (fig. I).

Nella seconda metà del secolo XIII e nel XIV gli scudi s'impiccoliscono; alti circa 2 piedi $1 / 2$ e larghi 9; hannò la forma triangolare a lati eguali e perciò detti scudi triangolari.

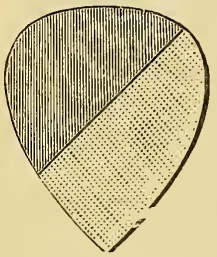

Fig. 1.

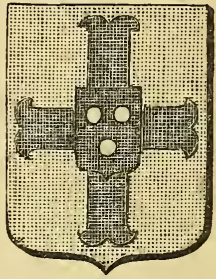

Fig. 2.

Nel XV secolo direntano retti ai lati, e al disotto rotondati, chiamati scudi semirotondi, dei quali più facilmente si accomodano le armi composte.

Usarono anche in questo tempo gli scudi a punta (targhe) adoperate nei tornei: fortemente incavati, piegati, e dal lato sinistro forniti di un intaglio, traverso cui nel giostrare passava la lancia.

Dal XVI secolo in poi venne in uso generalmente la forma cogli angoli inferiori arrotondati, e in fondo una punta, e questo scudo fu detto sannitico, francese o moderno (fig. 2). 
Si ottengono le proporzioni geometriche di questo scudo dividendo la sua larghezza in 7 parti uguali, e la sua altezza in 8 parti; gli angoli inferiori sono arrotondati di un quarto di cerchio; di cui il raggio è d'una mezza parte; due quarti di cerchio della stessa proporzione nel mezzo della linea orizzontale del basso si congiungono al di fuori formando la punta.

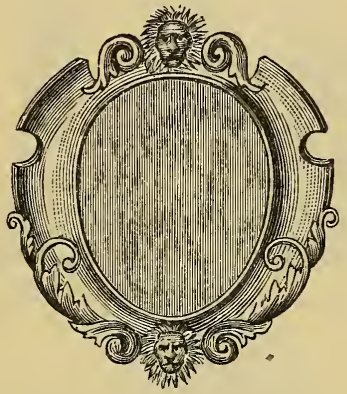

Fig. 3.

Nel risorgimento (renaissance) si diedero agli scudi gentilizi variatissime forme; ovali, rotonde, in vario modo intagliate, che poi nei secoli XVII e XVIII divennero affatto barocche.

Questi scudi presero il nome di targhe, targhe incavate, parme, rotelle, scudi perali, accartocciati, teste di cavallo, pavesi, clipei, pelte, cetre, ecc.

Lo scudo accartocciato (fig. 3) è circondato di arricciature e volute ornamentali, a gusto dell'artista, e lo si usa specialmente nei monumenti, nelle sculture, nelle 
miniature, ogni qualvolta si vuole armonizzare coi fregi e coi motivi architettonici o del disegno generale decorativo.

Gli antichi cavalicri banderesi portavano uno scudo quadrato detto bandierale o a bandiera (fig. 4), che fu in uso nei primi secoli dell'araldica fra i grandi signori del regno di Francia.

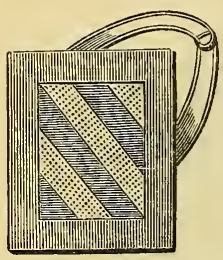

Fig. 4.

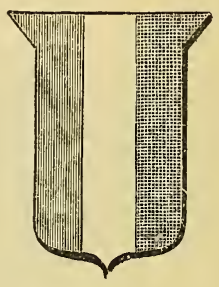

Fig. 5.

Quantunque non sempre nella storia dell'arte si riscontri vero, i blasonisti attribuirono gli scudi ovali agli Italiani, gli appuntati ai Francesi, le targhe ai Tedeschi, gli arrotondati agli Spagnuoli, i perali e le teste di cavallo ai Toscani, e gli scudi rettilinei inferiormente appuntati agl'Inglesi (fig. 5).

Viene notata dagli araldisti una particolarità degli scudi, che è l'inclinazione dei medesimi verso la parte destra, di maniera che l'angolo sinistro dello scudo comparisce sollevato sopra del destro (fig. 6).

Questa pendenza è frequentissima negli scudi antichi di qualunque forma o figura che siano.

Vogliono alcuni che lo scudo cosi cadente rappre- 
senti il cavaliere nell'atto del combattere; tanto più che sopra di questi scudi sogliono sempre collocarsi gli elmi chiusi quali si portavano in battaglia. Altri, come il Vulson, il Ménestrier, il Cellonese ne deducono l'origine dalle giostre, dai tornei ed altre imprese degli antichi cavalieri, i quali per mantenere il loro valore in

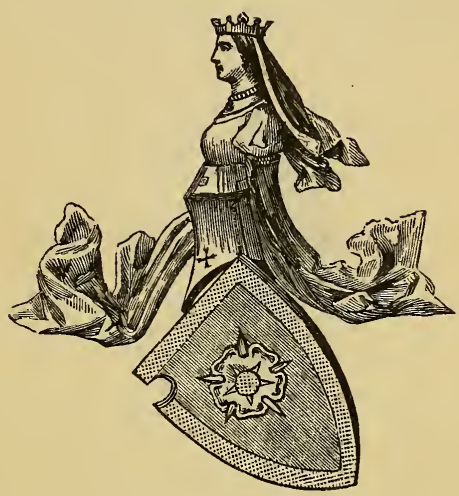

Fig.: 6.

tempo di pace, o giostravano, o sfidavano cavalieri erranti presso i ponti o altri luoghi di passaggio, appendendo il proprio scudo ad un albero a ad un palo; e tali scudi cosi affissi comparivano pendenti.

Il Cartari crede che più veramente l'uso di fare gli scudi inclinati si prendesse dal vedere che $\mathrm{i}$ soldati appendevano ai loro padiglioni i propri scudi aspettando il momento della battaglia; oppure quando in segno 
di vittoria innalzavano sopra dei pali gli scudi e le armi dei loro nemici che debellarono in battaglia.

A noi non pare gran cosa verosimile tale induzione, perciocchè nel secondo caso erano le armi dei nemici e non le proprie che ponevano in trofeo; e ci sembra da seguirsi l'opinione del Ménestrier.

Certo che è segnale di antichità lo scudo situato in quella guisa.

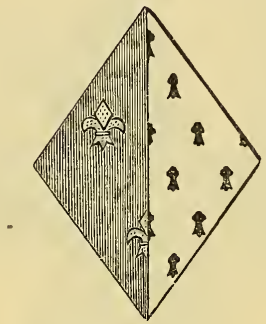

Fig. 7.

Le donne usarono ed usano una forma di scudo speciale, il quale ha la figura di un rombo geometrico (fig. 7).

Pare avesse origine dai cuscini sui quali trapungeano le loro armi, oppure dal fuso, simbolo dei casalinghi doveri donneschi. Lo scudo a losanga si circondava di lacci di amore se apparteneva a donzella, di cordelliera se era portato da una vedova. Ma per eccezione qualche principe o gentiluomo adottò questa forma di scudo, come fecero alcuni dei Visconti; e per eccezione ancora le donne figurarono le loro armi nello scudo san- 
nitico proprio dei cavalieri; in questa forma di scudo è scolpita l'arme sui monumenti e sulle medaglie di Giovanna d'Arco. Le donne accollano la propria arme a quella del marito. Si dice accollato lo scudo ad un altro, allorchè essi o si toccano colle punte, o sono congiunti sotto la stessa corona.

Lo scudo che affetta una configurazione quadrangolare ha quattro lati, detti capo in alto, punta in basso, fianco destro e fianco sinistro.

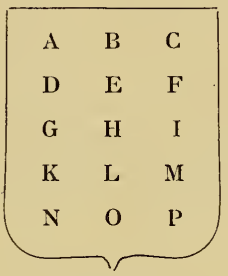

Fig. 8.

Si noti che in linguaggio araldico la destra dello scudo è la sinistra di chi lo guarda, e viceversa la sinistra è la destra dell'osservatore. Infatti figurandosi un cavaliere che abbia imbracciato lo scudo, la parte che ci sembra la sinistra sarà invece rivolta alla destra di chi lo porta.

Per facilitare il blasonamento dell'arma e l'esecuzione di questa dietro la descrizione araldica, gli araldisti hanno pensato di dividere idealmente lo scudo in tante parti, chiamate punti dello scudo, i cui nomi sono per lo più dedotti dalla comparazione dello scudo col corpo 
umano. La divisione più usata è quella del P. Ménestrier, rappresentata dalla fig. 8. - A è il canton destro del capo, B il punto del capo, C il canton sinistro del capo, D il punto destro del punto d'onore, $\mathrm{E}$ il punto d'onore, $\mathrm{F}$ il punto sinistro del punto d'onore, $\mathrm{G}$ il fianco destro, $\mathrm{H}$ il cuore o abisso, I il fianco sinistro, $\mathrm{K}$ il punto destro del bellico, L il bellico, $\mathrm{M}$ il punto sinistro del bellico, $\mathrm{N}$ il destro canton della punta, O la punta, $\mathrm{P}$ il canton sinistro della punta. Sotto ad $\mathrm{O}$ vi è la punta bassa o infima.

Mediante questo metodo si riconosce facilmente il collocamento e la disposizione delle pezze e delle figure. Per esempio il capo occupa lo spazio dei punti ABC, la fascia i punti GHI, la campagna i punti NOP, il palo i punti BEHLO, il canton-franco i punti AD, la croce i punti BEGHLLO, la pergola i punti ACHLO, ecc. 


\section{CAPITOLO III.}

\section{Smalti e Tratteggi.}

Chiamasi campo il fondo dello scudo, sul quale si disegnano le figure e le pezze. Questo vocabolo allude evidentemente al campo di battaglia su cui i cavalieri facevano le loro prove di valore. Esso è semplice o composto secondochè consta d'un solo o di più smalti.

Campi si chiamano poi tutte le tinte che possono coprire lo scudo, e sono sette smalti e due pelliccie.

Gli smalti si dividono in metalli e colori. Anticamente si ponevano sulle sopravvesti dei cavalieri le figure dell'armi fatte d'uno stagno battuto e smaltato di rosso, di verde, di nero e di turchino; ciò che fece dar loro il nome di smalti, e ne venne al blasone la legge della sovrapposizione degli smalti, cioè la proibizione di porre colore su colore o metallo su metallo.

I metalli sono l'oro e l'argento.

I colori sono il rosso, l'azzurro, il verde, il nero, e la porpora.

Gli altri colori non sono affatto usati in araldica, nemmeno il bianco e il giallo (sostituiti dall'argento e 
dall'oro), tranne quando le figure sono rappresentate $a l$ naturale, cioè colle tinte loro proprie. La tinta propria delle figure tratte dal corpo umano si chiama carnagione.

La porpora stessa è raramente usata nel campo dello scudo, e serve quasi esclusivamente pei padiglioni, manti, cappelli, ecc.

Altri colori, come il cannellato o tanné, l'aranciato, il lionato, il sanguigno non si riscontrano che in poche armi straniere, specialmente inglesi.

Quando le armi non sono dipinte, cioè nelle stampe, nei disegni, sul marmo, sul bronzo, ecc., gli smalti si rappresentano con segni convenzionali detti tratteggi (in francese bachures). É difficile precisare l'epoca in cui si fece conoscere un' invenzione cosi utile per l'arte araldica, dacchè senza introdurre nell'arme cifre o segni che ne potevano cambiare la configurazione o falsarne l'esattezza, questi tratteggi servono a far distinguere al. primo sguardo tutta la disposizione degli smalti in un' arma, ed anzi la rendono più elegante colle loro diverse ombreggiature.

Prima di tale invenzione furono in uso diversi sistemi, tutti difettosi. Gli Italiani e i Tedeschi nel descrivere le armi per indicare i colori si erano sempre serviti della prima lettera di ciaschedun colore. Cosi fecero l'Ughelli nell'Italia Sacra, il Franzoni nelle Armi delle famiglie nobili genovesi, il Borghini nel Discorso sulle armi fiorentine, il Mugnos per le famiglie siciliane, ecc.

Altri vollero significare colori e metalli colle prime sette lettere dell'alfabeto, ed altri coi primi sette numeri dell'aritmetica. 
Gli Inglesi andarono in cerca di stranezze: Spillmann nell'Aspilogia si serve di caratteri pianeti: Sol, oro; Luna, argento; Mars, rosso; Jupiter, azzurro; Mercurius, violato; Venus, verde, Saturnus, nero.

Ma tutti questi metodi caddero in disuso dinanzi a quello di cui disputansi l'invenzione il Pietrasanta, nelle sue Tessere gentilitice stampate in Roma nel 1638 ; il Butkens nella Genealogia dei Lynden edita nel 1626; il Wulson de la Colombière nel Cap. IV della sua Science

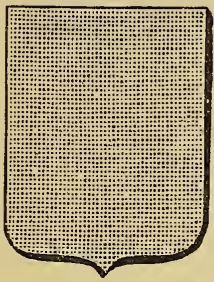

Fig. 9.

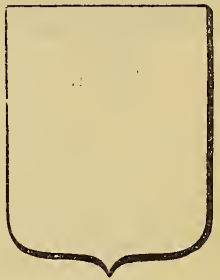

Fig. 10.

biroique, e l'alemanno Franquart, che il primo fece incidere sei piccoli quadri con tratteggi rappresentanti gli smalti, in un suo libro sui funerali dell'Arciduca Alberto d'Austria nel I622..

A descrivere le armi, esprimendo i colori di queste nelle loro figure, il blasonista si serve di semplici punti e linee diversamente tirate sopra le armi medesime.

Per rappresentare l'oro punteggiano lo scudo o quella parte o figura di esso che è di tale metallo (fig. 9).

Per l'argento lasciano il bianco (fig. Io).

Per esprimere il rosso tirano linee perpendicolari 
dalla parte superiore all'inferiore dello scudo (fig. I I).

L'azzurro indicano con linee orizzontali dall'uno all'altro lato dello scudo (fig. I2).

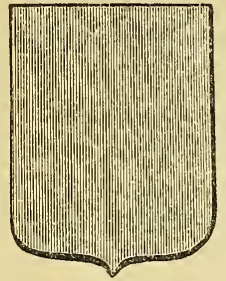

Fig. 11.

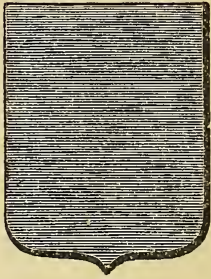

Fig. 12.

Il verde accennano con linee diagonali, tirate dall'angolo superiore destro alla parte inferiore sinistra dello scudo (fig. I3).

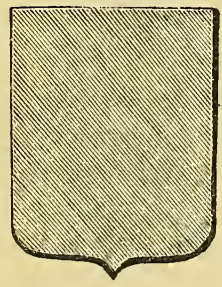

Fig 13.

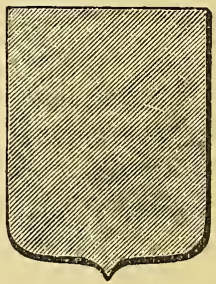

Fig. 14

Il violato o porpora addimostrano con linee parimente diagonali, ma tirate dall'angolo superiore sinistro verso la parte inferiore destra (fig. I4). 
Il nero formano con delle linee tirate in croce cioc orizzontali e perpendicolari insieme, (fig. I5), oppure facendo nero il campo o le figure (ciò che non può servire che per le stampe).

Il Sacken dà una regola importante da osservarsi nel tratteggio di uno scudo inclinato.

Il tratteggio, dice egli, deve sempre dirigersi secondo la direzione dello scudo e secondo il suo asse, di modo che le linee perpendicolari

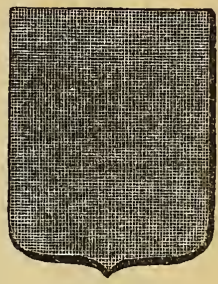

Fig. 15. indicanti il rosso corrono parallele all'asse longitudinale; le orizzontali indicanti l'azzurro parallele all'asse traversale, ossia all'orlo superiore allo scudo.

Si devono quindi prendere gli strati delle linee non già secondo la direzione della carta sopra la quale si disegna, ma secondo la direzione

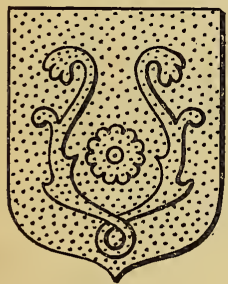

Fig. 16. dello scudo, conforme alla quale si dovrà eseguire il tratteggio.

$\mathrm{Vi}$ fu, specialmente in Germania, il costume di damaschinare lo scudo rabescando lo smalto dello stesso, o di diverso colore o metallo. Dicesi rabescato, o gallicamente diaprato (fig. I6). Il Ginanni lo chiamò anche con modo francico diaprato.

Questo lavoro ebbe lo scopo soltanto di ornare e di abbellire lo scudo.

Olttre i mealli e i colori, si considerano come campi 
anche due pelli, o pelliccie, o panni, o foderature, che sono l'armellino e il vaio.

Gli antichi araldisti chiamarodo anfibia la pelliccia, perchè è permesso nell'arte araldica di porre a piacere metallo o colore sopra la medesima.

L'armellino è una pelliccia bianca sopra la quale sono seminate le nere cime della coda di questo animale (fig. I7).

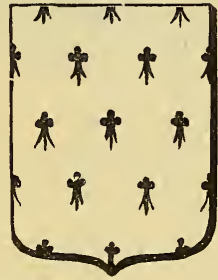

Fig. 17.

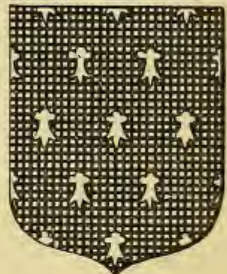

Fig. 18.

Gli araldisti italiani chiamarono i fiocchetti dei peli neri sparsi nell'armelliṇo moscature, in figura simili a crocette la di cui parte inferiore si spiega e si dilata in tre punte.

Se le moscature sono bianche sopra fondo nero, la pelliccia viene detta contrarmellino (fig. I8).

Quando l'armellino è composto di due smalti, altri che l'argento e il nero, ciò che si trova di rado, dicesi armellinato.

Il vaio è una foderatura composta di quattro fila di pezzi di argento in forma di campanelle rovesciate sopra un campo azzurro (fig. I9). 
Dicesi che le foderature di vaio siano tratte da pelli di martore zibelline o di rats musqués, che trovansi nella Moscovia.

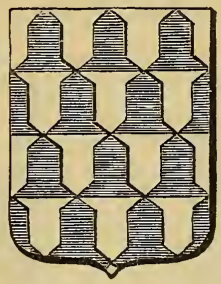

Fig. 19.

In Firenze l'arte dei vaiaj era una delle arti maggiori e più stimate; e quelle pelli non era permesso di portare indosso che ai cavalieri, ai dottori, e a gente di alto affare.

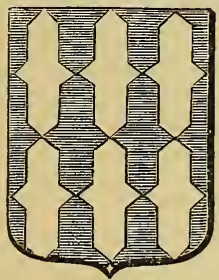

Fig. 20.

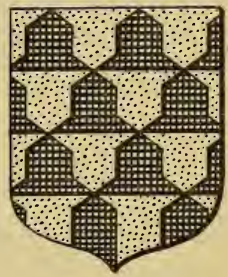

Fig. 21.

Il controvajo è una foderatura nella quale quelle figure in forma di campanelle sono opposte fra loro colle basi, e quando l'argento è opposto all'argento e l'azzurro all'azzurro (fig. 20). 
Quando il vaio è composto di due smalti diversi dagli ordinarii tali che oro e verde, argento e nero, oro e rosso, ecc., allora la pelle prende il nome di valato (fig. 2I); per la stessa ragione il controvajo si chia-

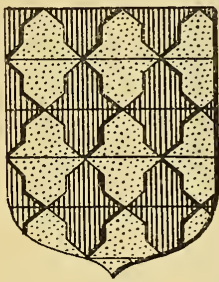

Fig. 22.

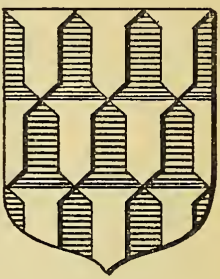

Fig. 23.

ma controvajato, quando non è d'argento e d'azzurro (fig. 22).

Il vaio che è di sei file anzichè di quattro chiamasi vaio minuto; se poi è di tre file sole, gran v'aio (fig. 23). 


\section{CAPITOLO IV.}

\section{PARTIZIONI E RiPARTIZIONI.}

Gli scudi che hanno il campo di un solo colore o di un solo metallo chiamansi scudi pieni; e pieni si dicono ancora quelli coperti da pelliccie senza figura.

Lo scudo può essere diviso in varì campi mediante una o più linee.

Tali partizioni si dicono semplici quando risultano da una sola linea, composte o meglio ripartizioni quando sono formate da due o più linee.

I. Le partizioni semplici sono: il partito, lo spaccato, il trinciato, il tagliato, l'addestrato e il sinistrato.

Partito, è lo scudo diviso in due parti uguali da una linea longitu-

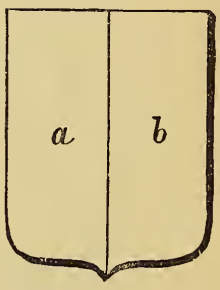

Fig. 24. dinale (fig. 24).

Spaccato o Troncato, quello diviso in due parti uguali da una linea orizzontale (fig. 25).

Trinciato, quando è diviso diagonalmente da una 
linea che dall'angolo superiore destro scende all'angolo inferiore sinistro (fig. 26).

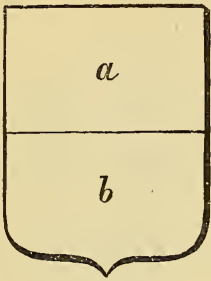

Fig. 25.

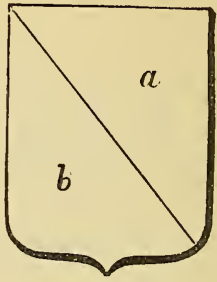

Fig. 26.

Tagliato, quando è diviso diagonalmente da una linea che dall'angolo superiore sinistro scende all'angolo inferiore destro (fig. 27).

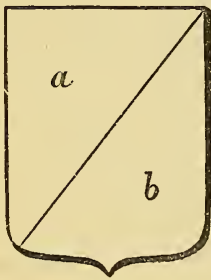

Fig. 27.

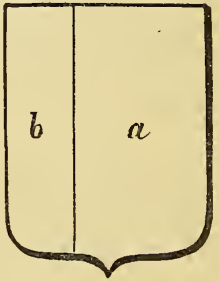

Fig. 28.

Addestrato, è lo scudo diviso perpendicolarmente da una linea non passante pel centro, in maniera che il bordo di smalto diverso, formato da essa alla destra 
dello scudo, non eccede il terzo della larghezza totale di esso (fig. 28).

Sinistrato, è quello in cui la linea non passante pel

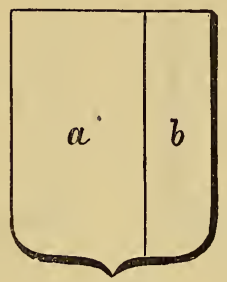

Fig. 29.

centro, forma alla sinistra dello scudo un bordo che non eccede il terzo della larghezza totale del medesimo (fig. 29).

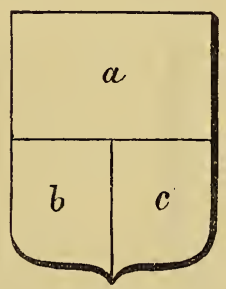

Fig. 30.

II. Le ripartizioni sono numerosissime. Noi accenneremo le principali.

Lo scudo spaccato, e partito nella sezione inferiore, dicesi spaccato-semipartito (fig. 30); se è spaccato e 
partito nella sezione superiore, semipartito-spaccato (figura 3I); se è partito, e spaccato nella sezione sinistra,

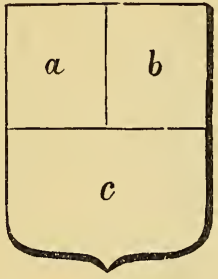

Fig. 31.

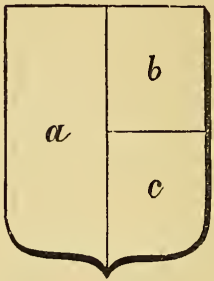

Fig. 32.

partito-semispaccato (fig. 32); se è partito e spaccato nella sezione destra semispaccato-partito (fig. 33).

Lo scudo può essere diviso in tre campi uguali mediante due linee parallele perpendicolari, orizzontali,

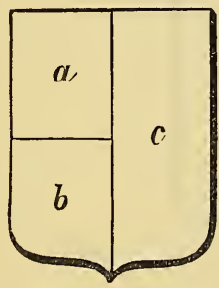

Fig. 33.

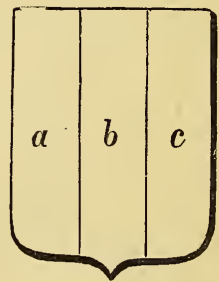

Fig. 34.

diagonali da destra a sinistra, o diagonali da sinistra a destra, che formano l'interzato in palo (fig. 34), in fascia (fig. 35), in banda (fig. 36), e in sharra (fig. 37). 
Inquartato, è lo scudo diviso in quattro spazi uguali per una linea perpendicolare ed una orizzontale che si incrociano (fig. 38).

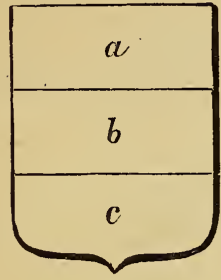

Fig. 35.

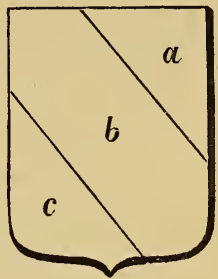

Fig. 36.

Quando queste due linee incrociate sono diagonali, lo scudo allora è inquartato in croce di Sant'Andrea o in decusse (fig. 39).

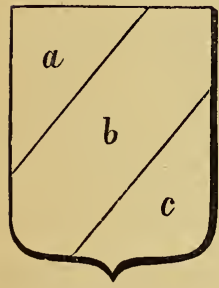

Fig. 37.

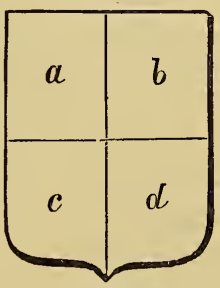

Fig. 38.

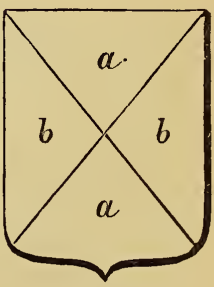

Fig. 39.

Avvertasi che nelle figure che diamo delle partizioni, le lettere $a, b, c, d$ indicano l'ordine col quale devono essere blasonate le rispettive sezioni del campo. 
Per estensione diconsi quarti tutte le porzioni di uno scudo diviso in quadrilateri uguali fra di loro, per mezzo di linee perpendicolari ed orizzontali, che s'incrociano. Per descrivere tali armi bisogna quindi contare il numero di queste linee, come per esempio: Partito di due, spaccato di due, il che dà nove quarti.

Partito di tre, spaccato di tre, il che dà sedici quarti (fig. 40).

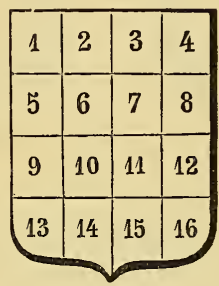

Fig 40 .

Partito di quattro, spaccato di tre, il che dà venti quarti, ecc.

Un quarto che è di nuovo inquartato dicesi contrinquartato.

Tali inquartature, ossia scudi composti di molte armi non vantano una origine molto antica; i più complicati s'incontrano in Germania.

Presentemente l'arma più complessa si reputa quella del re di Prussia, imperatore di Germania: lo scudo porta 48 quarti, dei quali tre sono partiti in altri due, più una campagna rossa per le regalie, più tre scudetti sul tutto, l'ultimo dei quali è spaccato di due armi; in tutto 56 blasoni in un solo stemma! 
Varie cause hanno prodotto gl'inquartamenti, e sono le alleanze, $\mathrm{i}$ feudi, le dignità, le concessioni, le padronanze, le pretensioni, ecc.

$\dot{E}$ da notarsi che in uno scudo inquartato l'arma gentilizia primitiva sta sempre nel primo quarto o sul tutto. Le concessioni hanno però la precedenza anche sull'arma di famiglia.

Oltre le dette partizioni e ripartizioni, ve ne sono altre in araldica molto difficili a blasonarsi; perchè le linee ripiegansi spesso in diverse direzioni.

Per descrivere queste partizioni, dette straordinarie, è necessario esaminare in qual senso le linee dividono il campo e a quali partizioni semplici somigliano separatamente.

Alcune di esse prendono il nome

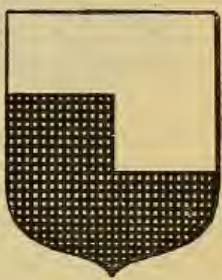

Fig. 41. dalle pezze araldiche o dalle figure di cui è data la forma dalle linee che dividono lo scudo.

Sono tutte rarissime nell'araldica italiana, e perciò ci limiteremo a menzionarle (I).

I. Semispaccato, semipartito verso la punta e rispaccato (fig. 4I).

2. Semispaccato in punta, semipartito verso il capo e rispaccato (fig. 42 ).

3. Semispaccato in capo, semitagliato verso la punta e rispaccato;

(1) Chi fosse curioso di avere la definizione di queste diverse partizioni straordinarie può consultare il Vocabolario Araldico di G. Guelfi (Manuali Hoepli). 
4. Semipartito, semitrinciato e ripartito (fig. 43).

5. Semipartito, semispaccato e ripartito (fig. 44).

6. Semispaccato, semitrinciato e rispaccato;

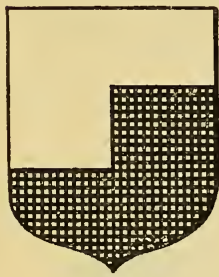

Fig. 42.

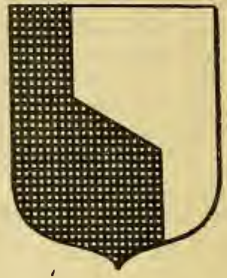

Fig. 43.

7. Semitrinciato, semitagliato e ritrinciato;

8. Semitagliato, semispaccato e ritagliato;

9. Semitagliato, semipartito e ritagliato;

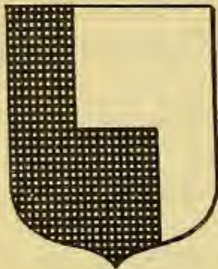

Fig. 44.

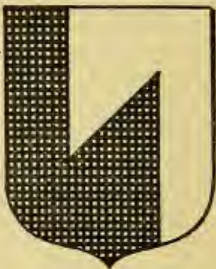

Fig. 45.

IO. Semitagliato, semitrinciato e ritagliato;

I I. Semitrinciato, semipartito e ritrinciato;

I2. Semipartito, semitagliato e ripartito (fig. 45). 
I3. Semipartito a sinistra, semitrinciato e ripartito (fig. 46).

I4. Interzato in calza (fig. 47).

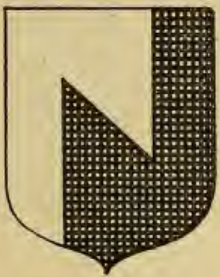

Fig. 46.

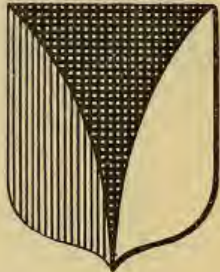

Fig. 47.

15. Interzato in grembo appuntato in sbarra (fig. 48).

16. Interzato-incappato (fig. 49).

I7. Interzato in capriolo (fig. 5o).

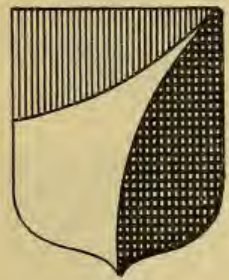

Fig. 48.

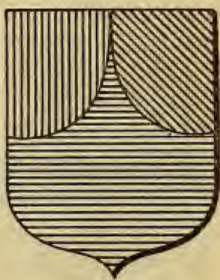

Fig. 49.

I8. Interzato in pergola (fig. SI).

I9. Interzato in mantello (fig. 52).

20. Interzato-abbracciato (fig. 53). 


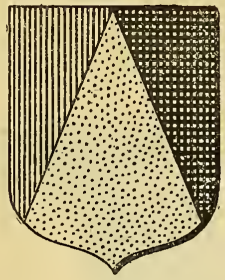

Fig. 50 .

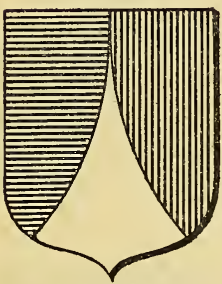

Fig. 52.

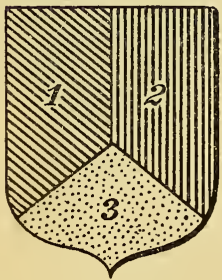

Fig. 5 .

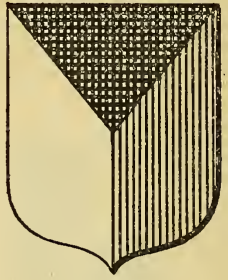

Fig. 51.

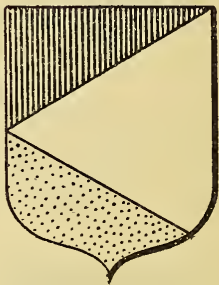

Fig. 53.

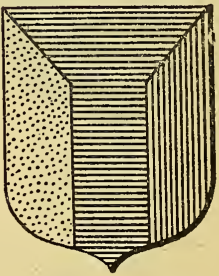

Fig. 55. 
21. Interzato in punta (fig. 54).

22. Interzato in gberone (fig. 55).

23. Interzato in grembi ritondati (fig. 56).

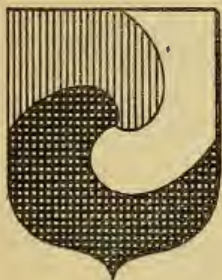

Fig. 56.

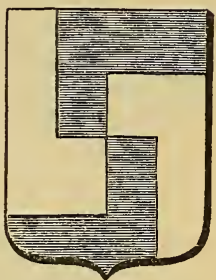

Fig. 57.

24. Inquartato in squadra (fig. 57).

25. Inquartato in grembi ritondati (fig. 58).

26. Diviso in capriolo;

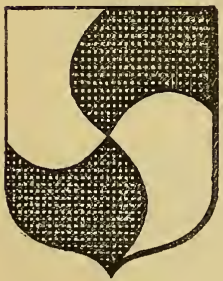

Fig. 58.

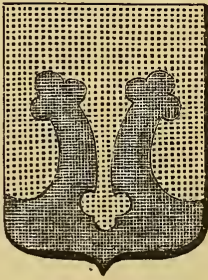

Fig. 59.

27. Diviso a lumaca;

28. Diviso a trifogli (fig. 59).

29. Diviso a teste di leone ingollate, ecc. 
Altre partizioni più bizzarre ancora si trovano nell'araldica tedesca.

Le linee delle partizioni possono essere modificate e spezzate in varie guise, e si diranno dentate, incliavate, increspate, innestate, merlate, merlettate, nebulose, ondate, scanalate, spinate, ecc. (I). Ne diamo alcuni esempi:

I. Inquartato-dentato (fig. 60);

2. Trinciato-dentato (fig. 6I);

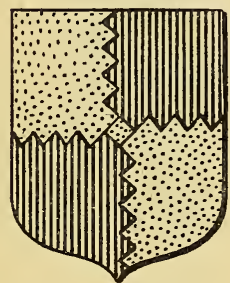

Fig. 60.

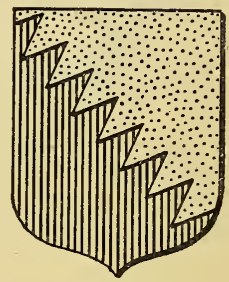

Fig. 61.

3. Partito-incbiavato (fig. 62);

4. Trinciato-inchiavato (fig. 63 );

5. Tagliato-inchiavato (fig. 64).

6. Inquartato, la linea orizzontale incliavata (figura 65).

7. Spaccato-merlato (fig. 66).

8. Partito-merlato (fig. 67).

(1) Per la definizione di questi attributi araldici, vedi il Vocabolario Araldico del Guelfi. (Manuali Hoepli). 


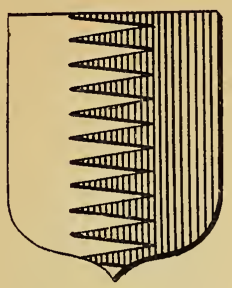

Fig. 62.

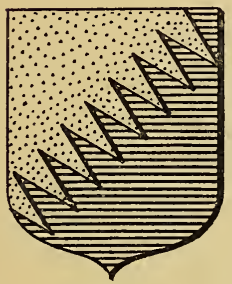

Fig. 64.

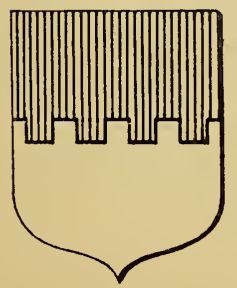

Fig. 66.

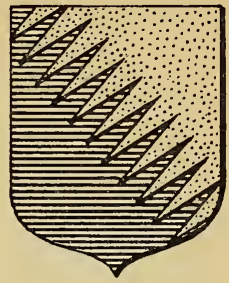

Fig. 63.

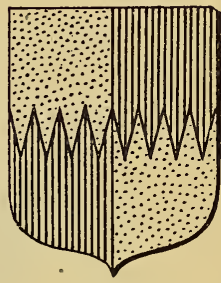

Fig. 65.

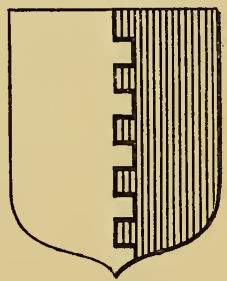

Fig. 67. 
9. Spaccato-ondato (fig. 68).

I0. Spaccato-nebuloso (fig. 69).

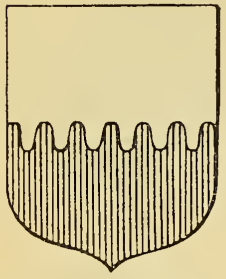

Fig. 68.

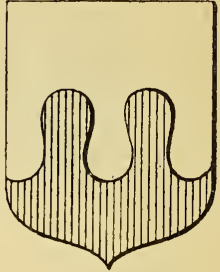

Fig. 69.

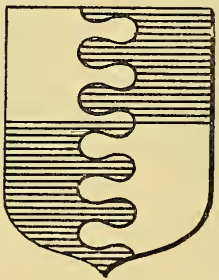

Fig. 70.

II. Inquartato, la linea perpendicolare nebulosa (figura 70). 


\section{CAPITOLO V}

Figure araldiche. - Pezze onorevoli.

Le figure araldiche, cioè proprie all'arte araldica si classificano in pezze onorevoli e in figure ordinarie. Sono le prime figure introdotte nell' armi, finchè in queste non comparvero i corpi animali, vegetali e minerali, e le figure artificiali. Si dicono onorevoli perchè si stimano le più nobili e considerate nel blasone, e quelle di cui l'uso è più frequente in tutti i paesi.

Non tutti gli araldisti si accordano nel numero e nella classificazione delle figure araldiche, e specialmente delle pezze onorevoli, che alcuni dividono in pezze onorevoli di primo e di secondo ordine. Il Brianville ne conta 9, il Paradisi Io, il Bombaci I2, il Menestrier I4, altri I6, altri sino a I9.

Senza soffermarci a suddivisioni troppo sottili perchè possano presentare un reale interesse, noi faremo conoscere quali sono le pezze onorevoli e quindi le figure ordinarie.

Il capo occupa il terzo superiore dello scudo, supponendolo diviso orizzontalmente in tre parti eguali (fig. 7 I ). 
Secondo l'opinione più diffusa, rappresenta l' elmo del cavaliere.

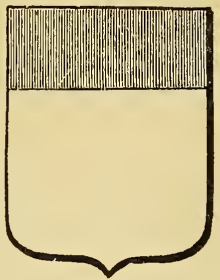

Fig. 71.

La fascia occupa orizzontalmente il terzo di mezzo dello scudo. Secondo il Pietrasanta rappresenta probabilmente il cingolo militare (fig. 72).

Se lo scudo sarà diriso da due linee perpendicolari in modo che lo dividano in tre parti uguali, lo spazio intermedio dicesi palo (fig. 73).

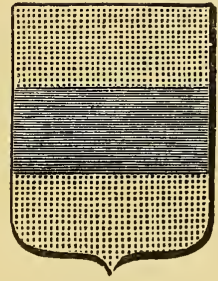

Fig. 72.

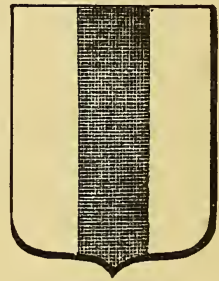

Fig. 73.

La banda è una figura diagonale che occupa la terza parte dello scudo stendendosi dall'angolo superiore de- 
stro alla parte inferiore sinistra del medesimo (fig. 74). La sbarra è la stessa figura della banda, colla diffe-

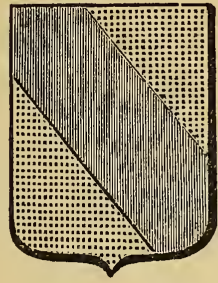

Fig. 74 .

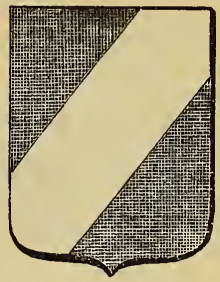

Fig. 75.

renza che la sbarra si stende dall' angolo superiore sinistro alla parte inferiore destra dello scudo (fig. 75).

Dalla combinazione del palo e della fascia si forma la croce (fig. 76).

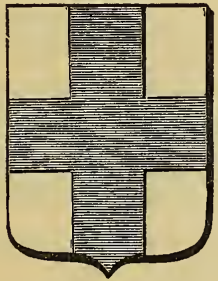

Fig. 76.

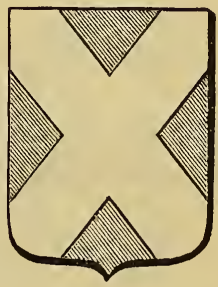

Fig. 77.

Dalla combinazione della banda e della sbarra si forma la croce di S. Andrea o decusse (fig. 77).

Il capriolo o scaglione si forma mediante la sbarra e 
la banda che muovono dagli angoli inferiori e si riuniscono nel punto di onore dello scudo (fig. 78 ).

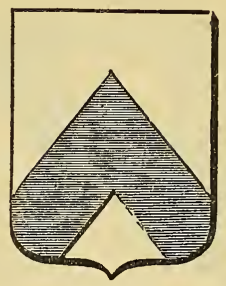

Fig. 78.

La fascia, il palo, la banda, la sbarra e il capriolo possono essere moltiplicati nello scudo, e allora devono essere di larghezza uguale a quella degli spazi intercedenti fra loro. Sei, otto, dieci fascie prendono il nome di burelle; cinque, sette, nove fascie si chiamano iran-

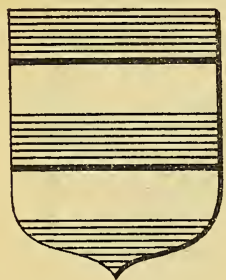

Fig. 79.

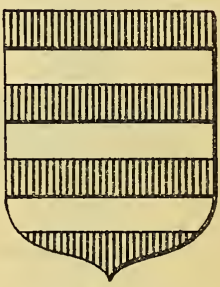

Fig. 80 .

gle. Due piccole fascie parallele ed accostate si dicono gemella; tre nella stessa disposizione terza. Esempi: alla fig. 79 due fascie; alla fig. 8o tre fascic; alla fig. 8I 
quattro fascie; alla fig. 82 una gemella; alla fig. 83 tre terze.

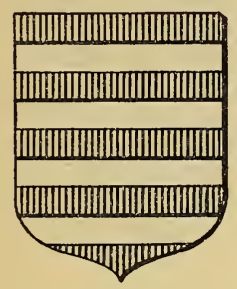

Fig. 81.

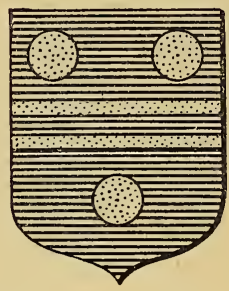

Fig. 82.

La divisa e la riga sono altresi fascie ristrette, ossia ridotte alla metà o a un terzo della ordinaria larghezza; la divisa e la riga non si mettono però mai in numero, ma sogliono attraversare altre figure o partizioni oppure sostenere un capo.

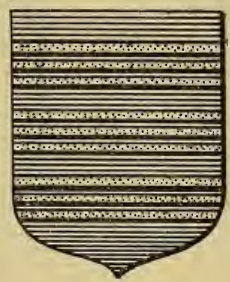

Fig. 83.

Un palo che occupa solo la metà della ordinaria larghezza prende il nome di verghetta o filetto in palo 
(fig. 84). Vedi alla fig. 85 tre pali, alla fig. 86 quattro pali.

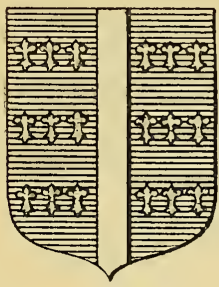

Fig. 84 .

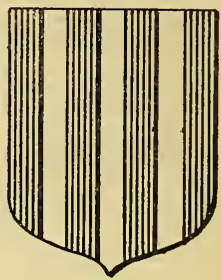

Fig. 85.

La banda ridotta alla metà della sua larghezza si chiama cotissa (fig. 87), ad un terzo bastone o filetto in banda (fig. 88). Vedi alla fig. 89 due bande, alla fig. 90 quattro bande, alla fig. $9 \mathrm{r}$, sei cotisse.

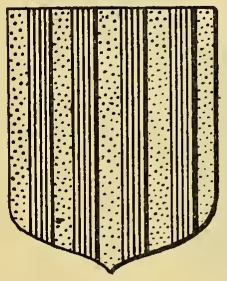

Fig. 86.

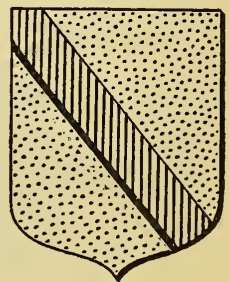

Fig. 87.

Anche la sbarra diminuita prende i nomi di traversa o controcotissa, controbastone, e controfiletto o filetto in harra. Vedi due sbarre alla fig. 92. 


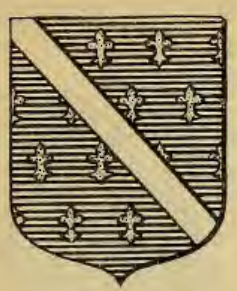

Fig. 88

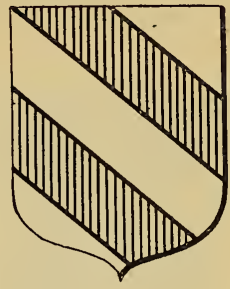

Fig. 89.

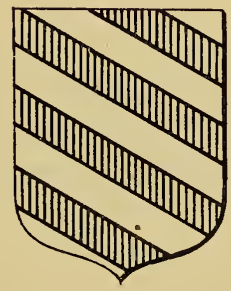

Fig. 90.

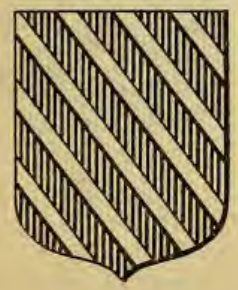

Fig. 91.

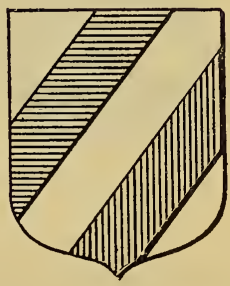

Fig. 92. 
Il capriolo diminuito dicesi scaglionetto o estaie (fig. 93). Vedi alla fig. 94 due caprioli, alla fig. 95 tre caprioli,

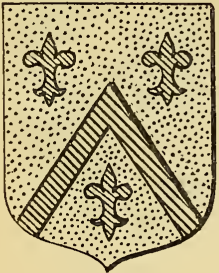

Fig. 93.

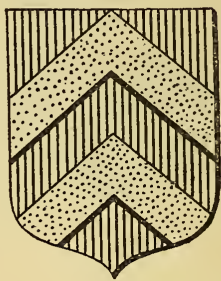

Fig. 94 .

alla fiig. 96 quattro caprioli, alla fig. 97 una gemella in capriolo o capriolo gemellato.

La croce diminuita prende il nome di filetto in croce o estrez (fig. 98).

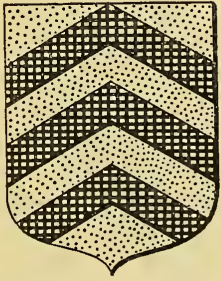

Fig. 95.

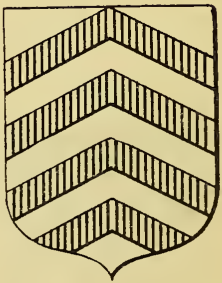

Fig. 96.

La campagna è la pezza che occupa la terza parte inferiore dello scudo, opposta al capo. 
Generalmente serve solo di sostegno a torri, castelli, animali, ecc., e si confonde spesso colla terrazza o pia-

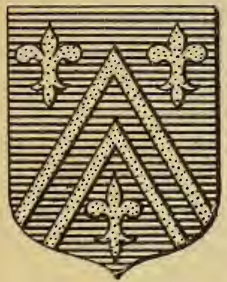

Fig. 97.

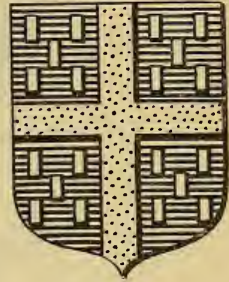

Fig. 98.

nura, tanto più che lo smalto più frequente della campagna è il verde (fig. 99).

La bordatura o bordura ì un'altra pezza onorevole che circonda tutto lo scudo occupando la terza parte

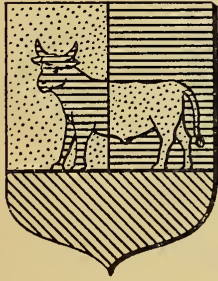

Fig. 99.

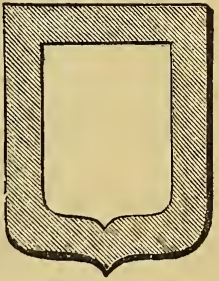

Fig. 100.

del campo. Il Ménestrier deriva la bordura dalle cotte d'armi orlate nei margini (fig. roo). 
La pergola è una figura simile al palo, che giunta al mezzo dello scudo si divide in due parti, terminan-

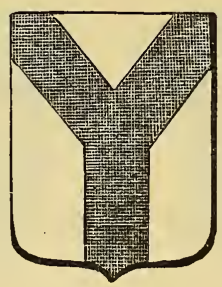

Fig. 101.

tisi ai due angoli superiori dello scudo in forma della lettera Y (fig. IOI).

Il gherone è una pergola in cui l'intervallo dei due bracci riuniti è pieno. Questa figura è molto rara (fig. 102).

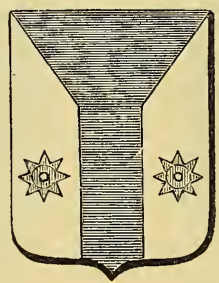

Fig. 102.

Quarto franco: questa pezza onorevole occupa uno spazio quadrato a destra del capo; la sua proporzione 
è in larghezza di tre parti delle sette dello scudo, e in altezza di tre parti e mezzo (fig. 103).

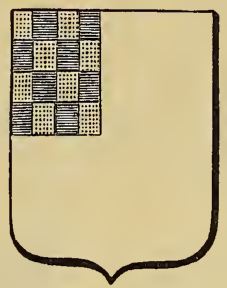

Fig. 103.

Il cantone è un quarto franco ristretto alla metà della sua grandezza ordinaria.

Il girone o grembo è una figura triangolare la cui base è larga la metà di un lato dello scudo, sul quale posa, ed ha il vertice nel centro. Probabilmente rap-

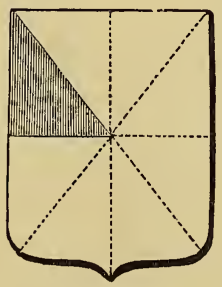

Fig. 104 .

presenta quelle stoffe tagliate a triangolo che le dame nel medioevo portavano intorno alla vita (fig. 104). 
La punta è un triangolo formato da due linee, che movendo dagli angoli inferiori convergono nel centro dello scudo, o poco più sotto (fig. IOS).

Se queste linee muovono dagli angoli superiori, allora la punta dicesi rovesciata.

La pila è un triangolo formato da due linee che movendo dall'angolo superiore dello scudo convergono nella punta di esso (fig. I06).

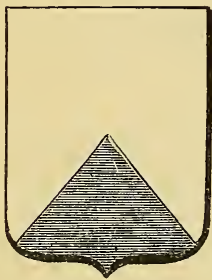

Fig. 105.

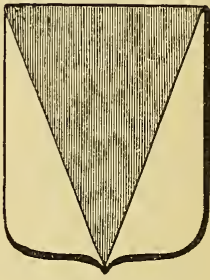

Fig. 106.

Lo scudetto, che da molti araldisti è considerato come una pezza onorevole di $2^{\circ}$ ordine, è un piccolo scudo posto nell' arma come qualunque altra figura. Spesso sta nel centro di un'inquartatura, e in questo caso porta generalmente l'arma primitiva della famiglia.

L'orlo o cinta è una bordura interna dello scudo, che lascia scoperti i bordi del medesimo (fig. 107). Esso ha $1 / 12$ della larghezza dello scudo, e si discosta dal bordo quanto è largo. La cinta può essere doppia, vale a dire gemella ed anche tripla.

Il piano è una campagna abbassata; il colmo, un capo 
alzato; il capo-palo è la riunione in un solo smalto del capo e del palo (fig. I08); la fasciacantone è la combinazione della fascia col cantone (fig. Iog); l'amaide, è una fascia che non tocca i lati dello scudo. Le amaidi

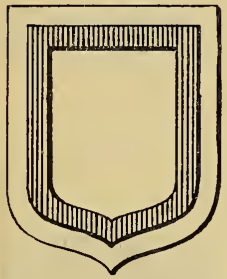

Fig. 107.

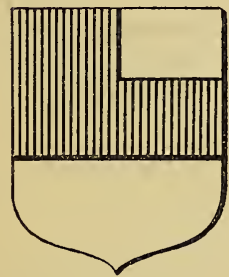

Fig. ${ }^{7} 109$.

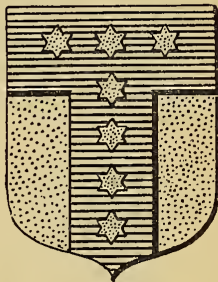

Fig. 108.

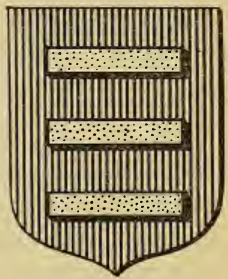

Fig. 110.

sono ordinariamente in numero di tre, l'una sull' altra (fig. I Io). Notiamo però che l'amaide non è considerata pezza onorevole che dai Francesi.

Tutte le pezze onorevoli possono venire alterate o modificate nelle loro linee, producendo così un gran numero di varietà che si riconoscono ai loro attributi, 
ossia ai termini tecnici che contraddistinguono queste modificazioni. Si hanno quindi pezze aguzzate, annodate, centrate o arcuate, contradoppiamerlate, contrafiammeggianti, contramerlate, dentate, doppiomerlate, finestrate, fiorenzate o gigliate, forate, inchiavate, increspate, ingollate, innestate, merlate, merlettate, nebulose, noderose, ondate, ritondate, rotte o frante, scanalate, spezzate o brisate, spinate, ecc. (I).

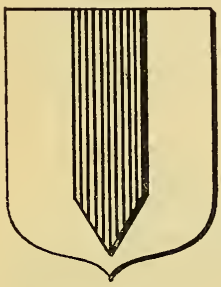

Fig. 111.

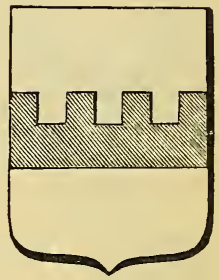

Fig. 112

Esempi: palo aguzzato (fig. I I I); fascia merlata (fig. II2;) due fascie contramerlate (fig. II3); palo doppiomerlato (fig. I I4); banda doppiomerlata (fig. II5); due fascie ondate (fig. II6); gemella in banda ondata (fig. II7); fascia nebulosa (fig. II8); banda spinata (fig. II9); fascia contradoppionoderosa (fig. I 20); fascia merlettata (fig. I2I); capo dentato (fig. I22); bordura dentata,detta anche filiera (fig. I23); tre fascie dentate inferiormente, dette anche foglie di sega (fig. I24); capo

(1) Per la spiegazione di questi attributi o termini araldici, rımandiamo lo studioso al Vocabolario. Araldico del Guelfi (Manuali Hoepli). 


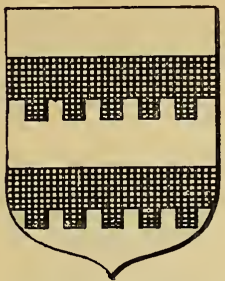

Fig. 113.

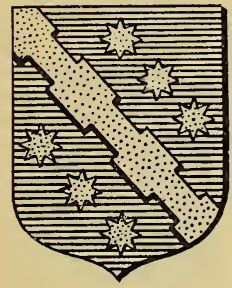

Fig. 115.

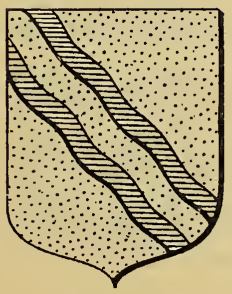

Fig. 117.

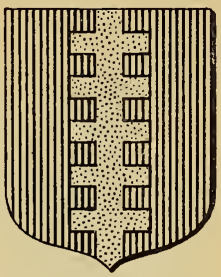

Fig. 114.

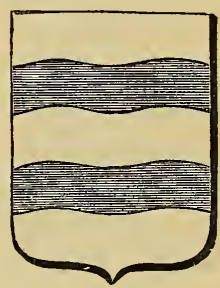

Fig. 116.

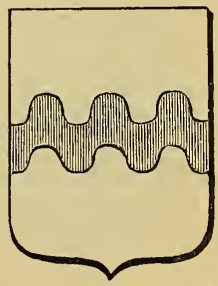

Fig. 118, 


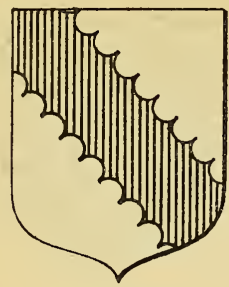

Fig. 119

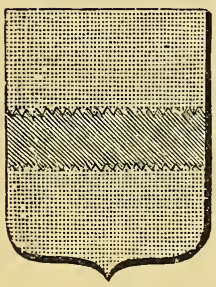

Fig. 121.

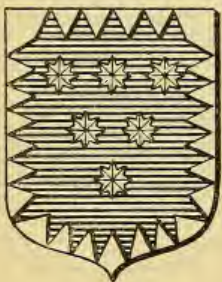

Fig. 123.

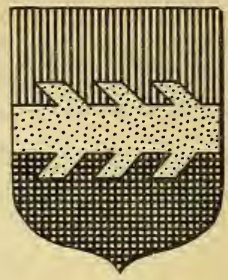

Fig. 120.

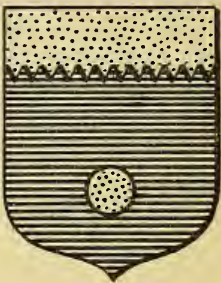

Fig. 122.

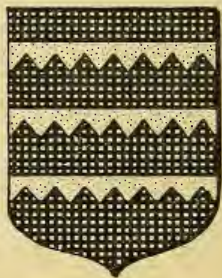

Fig. 124. 
inchiavato (fig. I25); bordura inchiavata di rosso e d'argento (fig. I26); doppia cinta controgigliata (fig. 127); capriolo rotto (fig. I 28 ), ecc.

Alcune pezze talvolta non raggiungono i lati dello

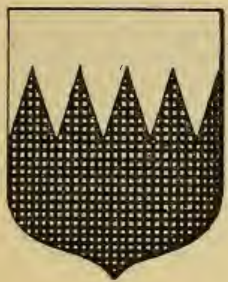

Fig. 125.

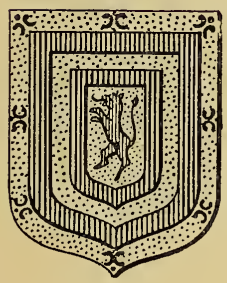

Fig. 127.

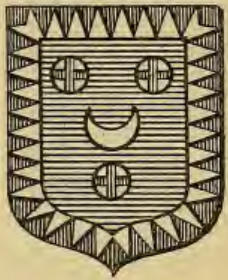

Fig. 126.

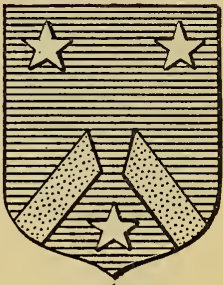

Fig. 128.

scudo, e si dicono scorciate se restano affatto isolate, ritirate se toccano un lato soltanto. Es.: capriolo scorciato (fig. I29); bastone scorciato (fig. I30); tre bande ritirate nel capo (fig. I3 I); tre pali ritirati sotto il capo (fig. I32). 
Altri attributi delle pezze onorevoli possono essere dati dalle figure che caricano, che accostano o che accompagnano le pezze stesse, o dalla loro posizione anormale. Quindi abbiamo pezze abbassate, alzate, accanto-

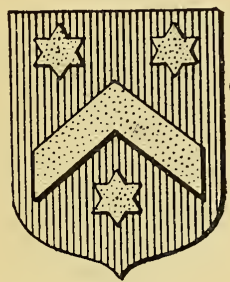

Fig. 129.

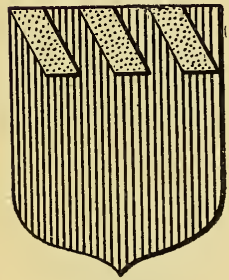

Fig. 131.

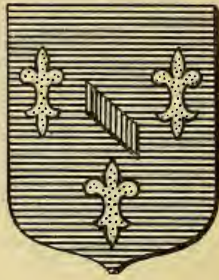

Fig. 130.

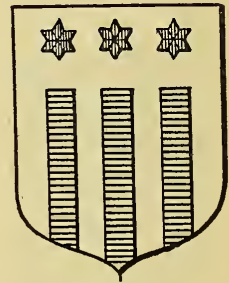

Fig. 132.

nate, accompagnate, accostate, addestrate, sinistrate, attraversanti, bisantate, bordate(fig. I33), composte (fig. I34), orlate, losangate, scaccate, sarchiate, sostenenti, sostenute, ripiene, vuote, ecc. (I).

(1) Vedi Vocabolario Araldico del Guelfi (Manuali Hoepli). 
La pezza araldica che si presta al maggior numero di trasformazioni è la croce, di cui i moderni araldisti hanno contato oltre a duecento varietà. Basterà accennare alle più conosciute: croce ancorata (fig. I35); croce

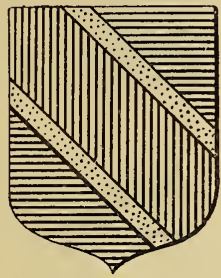

Fig. 133.

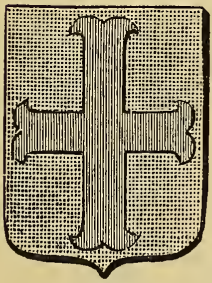

Fig. 135 .

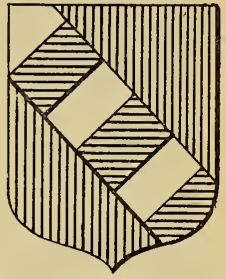

Fig. 134.

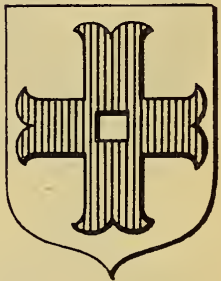

Fig. 136.

aperta in ferro di molino o mulinata (fig. 136); croce biforcata, o di Malta; croce bordonata o pomata (fig. 137); croce cancellata (fig. 138); croce doppia, o patriarcale o di Lorena (fig. I39); croce cordonata; croce dentata (fig. I40); croce falcata; croce fioronata; croce fitta; croce forchettata; croce fusellata; croce gigliata; croce 


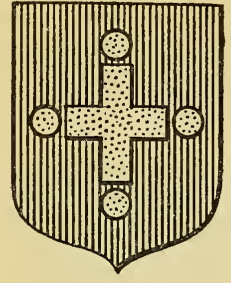

Fig. 137.

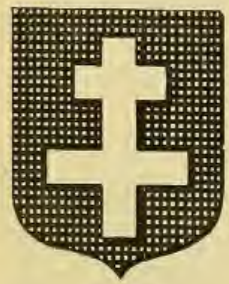

Fig. 139.

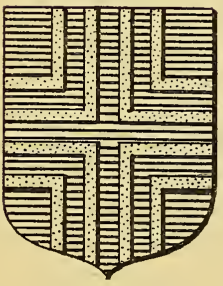

Fig. 141.

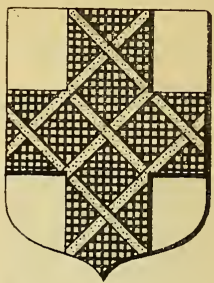

Fig. 138.

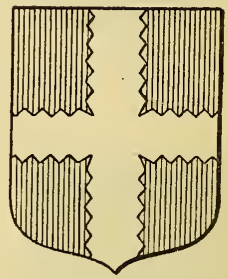

Fig. 140.

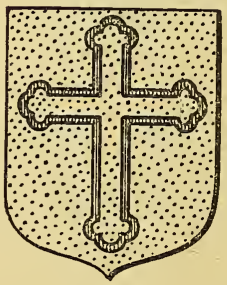

Fig. 142. 
gemellata (fig. I4I); croce increspata; croce ingollata; croce latina o del Calvario; croce latina trifogliata; croce latina vuota e trifogliata (fig. I42); croce merlettata; croce noderosa (fig. I43); croce ombrata a filetto;

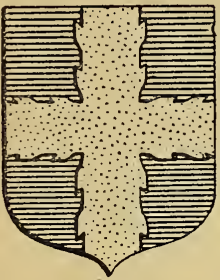

Fig. 143.

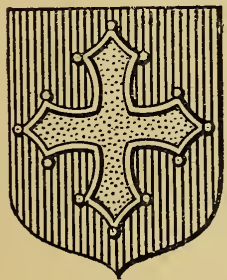

Fig. 145.

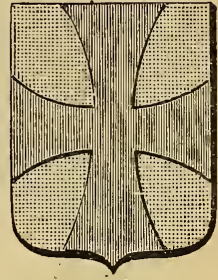

Fig. 144.

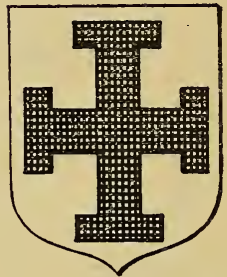

Fig. 146.

croce ondata; croce patente (fig. 144); croce patente gigliata; croce patente ritrinciata e pomettata o croce di Tolosa (fig. I45); croce potenziata (fig. I46); croce raggiata; croce ricrocettata; croce scalinata; croce scanalata; croce scorciata; croce semipotenziata; croce serpentifera; croce serpentina; croce spinata; croce trifogliata; ecc. 
Parecchie di queste alterazioni sono irregolari o addirittura bizzarre, come la croce doppia semipotenziatc a sinistra nella traversa inferiore della famiglia Tschet-

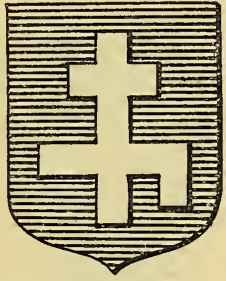

Fig. 147.

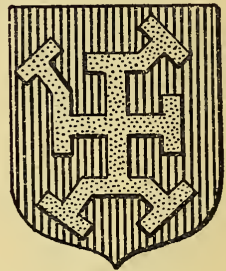

Fig. 148.

scke (fig. 147), e quella della famiglia Squarciafichi (fig. 148), per la quale occorre tutto l' accorgimento di un provetto araldista onde blasonarla esattamente. 


\section{CAPITOLO VI.}

\section{FiguRE ARALDICHE ORDINARIE.}

Le principali figure araldiche ordinarie sono la losanga, il fuso, il bisante, la torta, il plinto, il lambello e l'anelletto.

La losanga o rombo è una figura geometrica che ha due angoli acuti e due ottusi, e che è posta ordinariamente sopra uno degli angoli acuti. Essa differisce dal fuso inquantochè questo è più allungato. Nella fig. I 49 le tre figure del capo sono losanghe, le tre della punta sono fusi.

Dicesi losanga forata quella che ha un foro tondo nel mezzo, a traverso del quale si vede il campo dello scudo. Dicesi losanga vuota o maglia quella che è forata nel

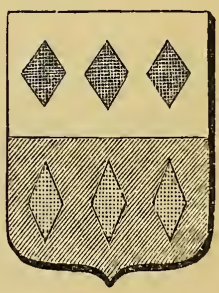

Fig. 149. mezzo secondo i suoi contorni, ossia che è caricata di altra losanga del campo. Nella figura I50, si vede una losanga forata a destra, e una losanga v'uota a sinistra. 
I bisanti: sono chiamate cosi tutte le figure tonde di oro e di argento che si prendono per monete; ancorchè in esse non comparisca nessuna impronta.

I bisanti o bisantini furono per la prima volta coniati a Bisanzio. Si vuole fossero introdotti in Europa dopo la presa di Costantinopoli fatta dai crociati.

I bisanti che sono di colore invece che di metallo si dicono torte o tortelli:

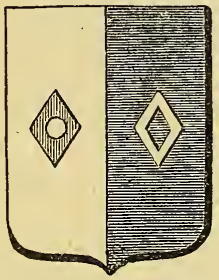

Fig. 150.

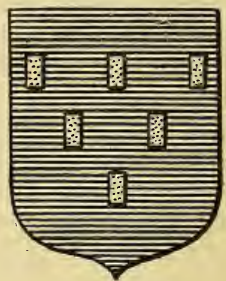

Fig. 151.

Bisante-torta o torta-bisante è quella che è divisa egualmente in metallo e colore, o colore e metallo.

Il plinto è una figura quadrilatera più lunga che larga in forma di mattone, e pare voglia alludere alle pietre colle quali furono fabbricati i castelli feudali (fig. I5I).

Il lambello fu denominato dagli araldisti italiani $r a-$ stello. o rastrello credendolo uno strumento di agricoltura. Il lambello proviene dal vocabolo gallico label o nodo di nastri che si attaccava all'elmo e serviva a distinguere i figli dai padri loro: il lambello viene reputato la più nobile brisura blasonica. Il lambello, nota 
il Ginanni, fu introdotto in Italia alla venuta di Carlo d'Angiò (nel 1265), e fu distintivo di parte guelfa, e concesso dai re di Napoli alle famiglie a loro devote insieme coi gigli d'oro nel capo d'azzurro.

Il Crollalanza definisce cosi il lambello: pezza araldica formata come una trangla scorciata e munita di pezzetti pendenti, che sono ordinariamente tre: nel caso che fossero in numero maggiore o minore conviene blasonarli.

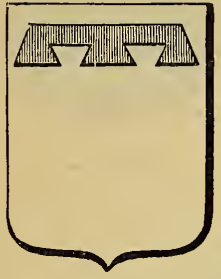

Fig. 152.

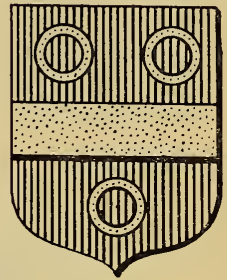

Fig. 153.

Il lambello ha 3 parti di lunghezza e $3 / 4$ di parte di altezza, di cui $1 / 4$, per la trangla e una e mezza per i pendenti. Esso si pone sempre in capo (fig. I52).

L'anelletto (fig. I53) è una figura araldica in forma di cerchio che negli scudi è sempre in numero.

Fra le altre figure araldiche dobbiamo tener conto delle seguenti.

Il ciclamoro è un grande anello che si trova sempre solo negli scudi (fig. I 54).

Le armille o circoli concentrici (fig. I55) si trovano in due $o$ in tre. 
Il quadro è uno scacco isolato nello scudo; può essere anche vuoto. Lo stesso dicasi del triangolo.

Il crancelino (fig. I56) è una banda centrata ossia arcuata, ornata di foglie di ruta superiormente, e rappresenta un cerchio di corona spezzata.

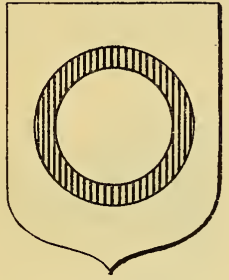

Fig. 154.

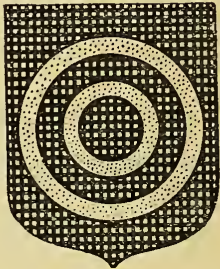

Fig. 155.

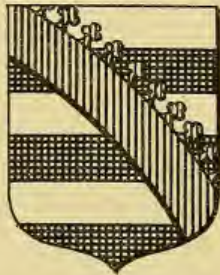

Fig. 156.

La potenza o tan è una figura simile a un $\mathrm{T}$; e la semipotenza è una potenza con un solo braccio, una specie di squadra.

Il raggio di carbonclio è una pietra preziosa che si rappresenta come una ruota, senza cerchio, di otto 
raggi gigliati, che muovono da un anelletto gemmato (fig. I57).

Le mandorle pelate, dette dai Francesi otelles, formano una figura araldica in cui sono sempre disposte in numero di quattro come nella fig. I 58 . $\grave{E}$ ormai provato

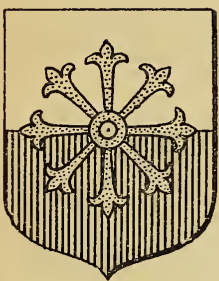

Fig. 157.

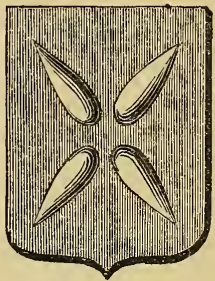

Fig. 158.

che non sono che un'alterazione della croce patente degli antichi sigilli dei conti di Comminges.

Il pentalfa è la figura volgarmente conosciuta col nome di segno di Salomone, ossia l'intreccio di due triangoli vuoti.

L'araldica straniera, e specialmente la tedesca, è ricca di altre figure meno comuni, che troppo lungo sarebbe enumerare. 


\section{CAPITOLO VII.}

\section{Convenevoli partizioni.}

Spesso le pezze onorevoli ed alcune figure araldiche ordinarie riempiono lo scudo dividendolo in due smalti alternati, aventi ciascuno la forma di essi pezzi, e formano in tal modo le partizioni chiamate dagli araldisti convenevoli.

Tali sono il fasciato, il palato, il bandato, lo sbarrato, il burellato, il verghettato, il cotissato, il contracotissato, il capriolato, il grembiato, lo scaccato, il losangato e il fusato, e le loro varietà.

Quando le partizioni sono composte di sei fascie, di sei pali, di sei bande, di sei sbarre, di sei caprioli o di otto grembi, non se ne blasona il numero; bensi quando sono in numero maggiore o minore.

Esempi: fasciato d'oro e di rosso di 4 pezzi (fig. 159); fasciato d'argento e di nero (fig. I60); fasciato d'oro e di rosso di 8 pezzi (fig. I6I); burellato d'argento e d'azzurro di ro pezzi (fig. 162); burellato d'oro e di rosso di 12 pezzi (fig. I63); palato d'oro e d'azzurro di 4 pezzi (fig. 164); palato d'argento e di rosso (fig. 165); 


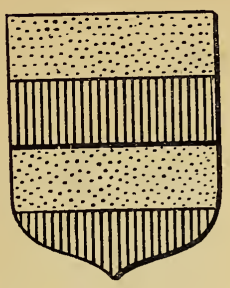

Fig. 159.

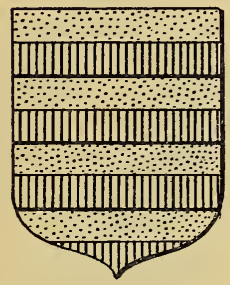

Fig. 161.

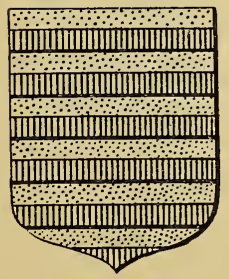

Fig. 163.

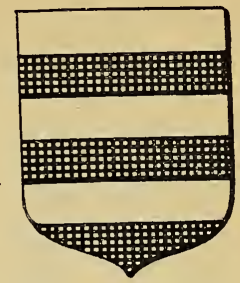

Fig. 160.

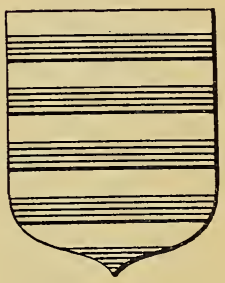

Fig. 162.

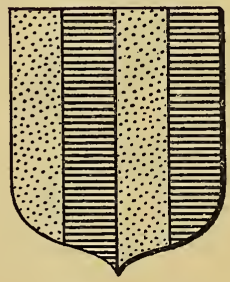

Fig. 164. 
palato d'argento e di rosso d'S pezzi (fig. I66); bandato di rosso e d'argento di 4 pezzi (fig. 167); bandato d'argento e di rosso di $\delta$ pezzi (fig. I68); cotissato d'argento

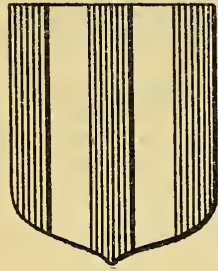

Fig. 165.

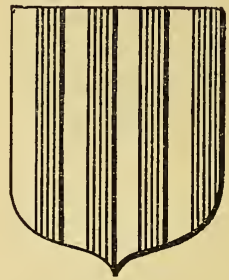

Fig. 166.

c d'azzurro di Io pezzi (fig. 169); cotissato di rosso $c$ d'oro di 12 pezzi (fig. I70); sbarrato di nero e d'argento di 4 pezzi (fig. I7I); sbarrato d'argento e di rosso

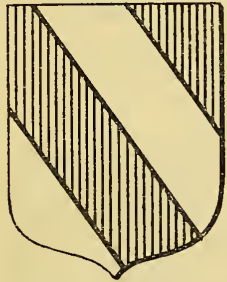

Fig. 167.

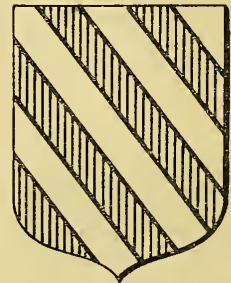

Fig. 1i:8.

di 8 pezzi (fig. I72); contracotissato d'argento e di porpora di Io pezzi (fig. I73); capriolato d'oro e di nero (fig. I74); grembiato di nero e d'argento (fig. I75); 


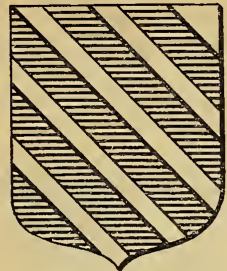

Fig. 169.

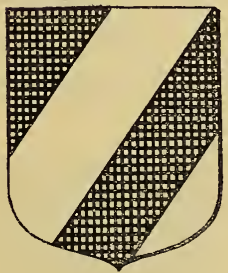

Fig. 171.

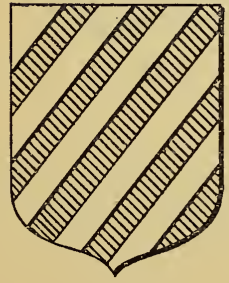

Fig. 173.

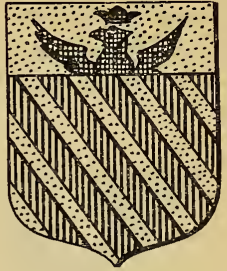

Fig. 170.

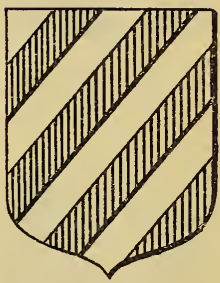

Fig. 172.

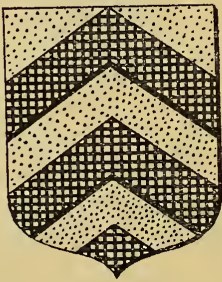

Fig. 174. 
grembiato-partito d'argento e di nero di 6 pezzi (fig. I76); grembiato-spaccato di rosso e d'argento di 6 pezzi (fig. I77); grembiato in decusse di rosso e d'oro (fig. I78); grembiato di rosso e d'oro di I2 pezzi (fig. I79).

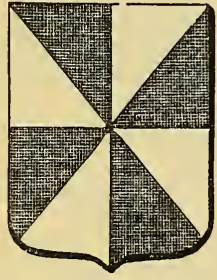

Fig. 175.

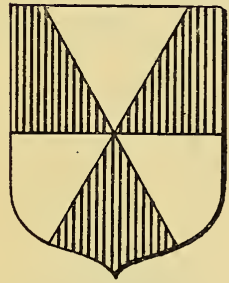

Fig. 177.

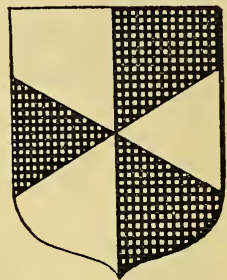

Fig. 176.

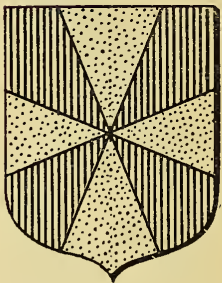

Fig. 178.

Lo scaccato è un campo che presenta l'imagine di uno scacchiere; deve avere sei file orizzontali, formando 36 caselle o scacchi; se ha un numero maggiore di caselle, bisogna indicarlo. Es. scaccato d'azzurro e d'argento di 7 file (pag. I80). 
Lo scudo scaccato di 5 file, formanti is caselle, prende il nome di punti di scacchiere (fig. I8I); di 3 file formanti 9 caselle, punti equipollenti.

Lo scudo coperto interamente di losanghe o di fusi

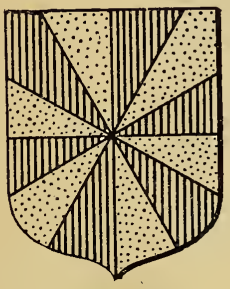

Fig. 179.

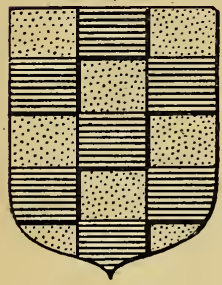

Fig. 181,

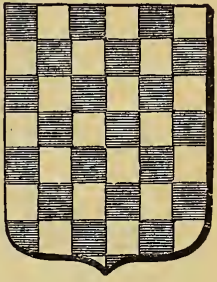

Fig. 180.

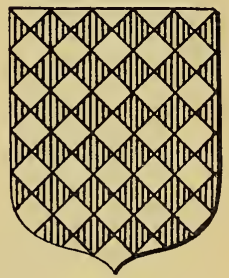

Fig. 182.

alternati di due smalti chiamasi losangato (fig. I82) e fusato (fig. I83). Vi è anche il fusato in band.a (fig. I84) e il fusato in sbarra (fig. I85).

Uno scudo coperto di triangoli alternati di due smalti. dicesi triangolato. 
Le convenevoli partizioni sono contrarianti quando le pezze che le compongono sono partite, spaccate, trinciate o tagliate, in modo che ciascuna metà opponga all'altra metà lo smalto contrario, alternativamente.

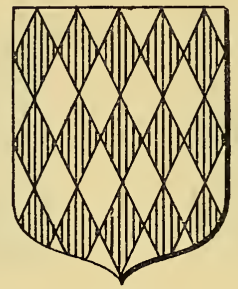

Fig. 183.

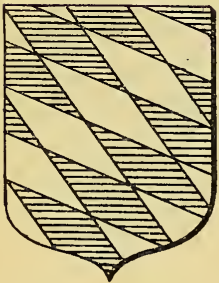

Fig. 184 .

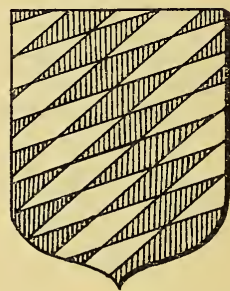

Fig. 185.

Es.: partito-contrafasciato d'oro e di nero (fig. I86); spaccato-contrapalato d'azzurro e d'argento (fig. 187); partito-contrabandato d'azzurro e d'oro (fig. I88); trinciato-contrasbarrato d'argento e di rosso (fig. I89).

Raramente le convenevoli partizioni sono di tre o più 


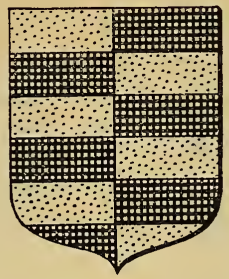

Fig. 186.

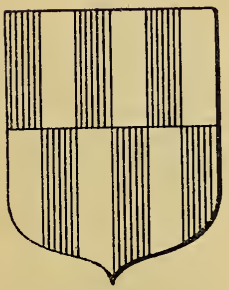

Fig. 187.

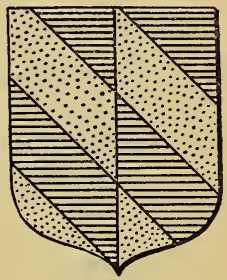

Fig. 188.

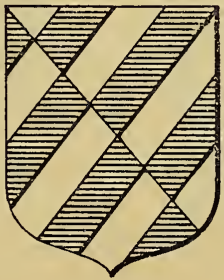

Fig. 189. 
smalti alternati. Es.: fasciato d'oro, d'azzurro e d'argento di 9 pezzi (fig. I9o).

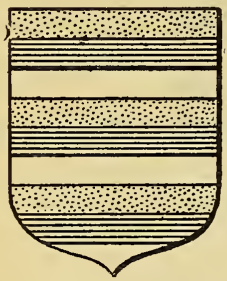

Fig. 190.

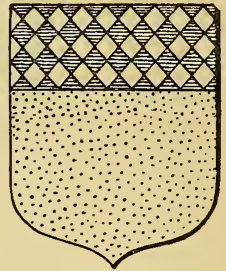

Fig. 191.

Anche le pezze onorevoli o le figure araldiche possono essere caricate di convenevoli partizioni; per es.: un capo losangato (fig. I9I), un palo bandato (fig. 192), un palo capriolato (fig. I93), ecc.

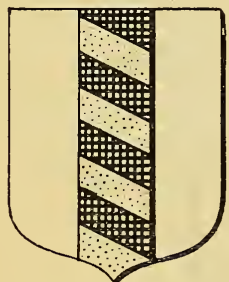

Fig. 192.

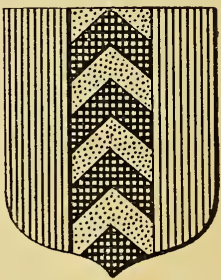

Fig. 193.

Molti degli attributi modificanti che si applicano alle pezze onorevoli alterano anche le linee divisionali delle convenevoli partizioni. Es.: bandato-nebuloso d'argento 


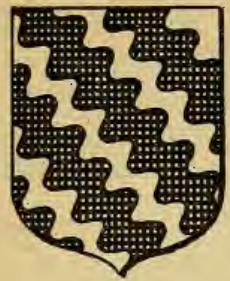

Fig. 191.

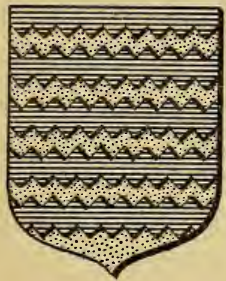

Fig. 196.

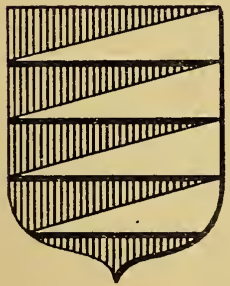

Fig. 198.

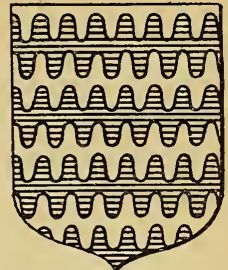

Fig. 195.

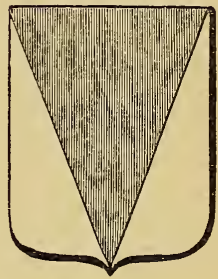

Fig. 197.

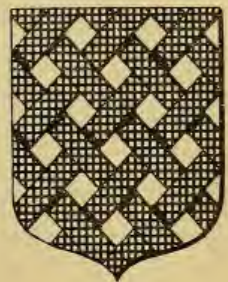

Fig. 199. 
e di nero di 8 pezzi (fig. I94); fasciato-innestato d'argento e d'azzurro di \& pezzi (fig. I95); burellato-increspato d'azzurro e d'oro di io pezzi (fig. I96).

Impropriamente alcuni araldisti considerano come convenevoli partizioni anche l'incappato, il calzato (figura 197), l'inchiavato (fig. I98), il cancellato (fig. 199) e l'inferriato (I).

(1) Per le definizioni di tutti questi termini, vedi Vocabolario Araldico del Guelfi. (Manuali Hoepli). 


\section{CAPITOLO VIII.}

Figure naturali, artificiali e chimeriche.

Chiamansi figure tutti quei corpi che si figurano in uno scudo.

Il Ginanni le distingue in quattro specie, araldiche, naturali, artificiali e chimeriche.

Fra queste figure non si annoverano tutte quelle che sono dichiarate pezze araldiche, le quali si differenziano anche dalle figure, in quanto conviene che queste siano situate libere nel campo almeno da due lati, nè possono estendersi fino ai margini dello scudo.

Abbiamo dato la classificazione del Ginanni: spieghiamola.

Le figure araldiche, di cui abbiamo già parlato, sono proprie di questa arte, e non si possono spiegare senza adoprare i termini blasonici.

Le naturali, rappresentano $\mathrm{i}$ corpi che si trovano in natura e nei suoi diversi regni, non che nel cielo, come animali, minerali, astri, ecc.

Le artificiali, sono quelle fabbricate dall'arte e industria degli uomini, e provengono o dal feudalismo, o 
dalla religione, o dalle arti e dai mestieri, e via dicendo.

Le figure chimeriche o fantastiche, sono quelle create dalla umana fantasia, come i draghi, i grifi, i liocorni, ecc.

Queste figure, specialmente le naturali, non devono essere al vero rappresentate come in natura, ma con un certo stile propriamente araldico, formante quello che dicesi tipo araldico.

Questo stile è differente a seconda dei tempi e dei paesi, e combina collo stile dominante dell'epoca, specialmente dell'architettonico, poichè sulle chiese, sui palazzi si solevano innalzare le armi nazionali e gentilizie; e perciò è da raccomandarsi agli artisti araldici, che conservino $\mathrm{i}$ tipi che sono chiamati a riprodurre colla loro arte (I).

FIGURE UMANE.

Si possono rappresentare nello scudo figure di uomini, di donne, di fanciulli, di santi e di sante, di angeli, di mori, di selvaggi, di divinità pagane; e possono ugualmente esservi figurate le singole parti della persona, come teste, busti, braccia, mani, cuori, costole, occhi, ecc.

Le teste possono essere ornate di svariate corone, di cappelli, di berretti, di tocchi.

Le teste di moro si rappresentano nere, e quando

(1) Vedi Crollalanza, Les Animaux du Blason. 
hanno un tortiglio di differente colore intorno alla testa di un altro smalto si blasonano attortigliote.

I busti sono di uomini e di donna, e si compongono della testa e del petto.

Le braccia sono dette destre o sinistre, secondo che sporgono nello scudo dal lato destro o sinistro.

Un braccio destro nudo, vestito o armato movente dal fianco sinistro dello scudo, chiamasi destrocherio, che era anticamente un'armilla o braccialetto che gli uomini o le donne portavano al polso destro; l'opposto dicesi sinistrocherio.

Quando blasonando si dice mano senz'altra aggiunta, $s$ 'intende una mano destra coll' indice o col medio sporgente, e prende il nome di mano giurante; se ella è sporgente dalle nubi o circondata da un nimbo, si chiama benedicente.

Due mani unite che si stringono prendono il nome di fede.

La triquetra, simbolo della Sicilia, forse alludente alle tre punte della Trinacria, è un emblema consistente in tre gambe unite in un centro formato da un volto umano, che sembrano roteare intorno ad un circolo invisibile.

Fra le figure tratte dal corpo umano si considerano anche le lagrime e le goccie di sangue.

\section{QUADRUPEDI.}

Gli animali appariscono le figure più nobili del blasone.

Essi si dividono in varie classi: quadrupedi, volatili, pesci, rettili, insetti, molluschi, figure chimeriche. 
$\mathrm{Si}$ pongono negli scudi anche le sole membra, cioè: teste, zampe, artigli, code recise, strappate e sanguinose.

Gli animali, come tutte le altre figure blasoniche, si possono dipingere di qualunque smalto, anche contrario al loro colore naturale.

Le pose dei quadrupedi sono diverse.

La più comune si è quella che li rappresenta ritti sulle gambe posteriori, una zampa innanzi all' altra, colle anteriori alzate disegualmente, di maniera mostrino tutte e quattro le zampe.

Si dirà l'animale passante, quando porta la zampa anteriore alzata: corrente, quando è in atto di correre, rampante ritto sulle zampe di dietro in atteggiamento di arrampicarsi.

Il cavallo rampante dicesi spaventato od inalberato, l'orso levato, il lupo rapace, il toro furioso, la capra saliente, il leopardo illeonito.

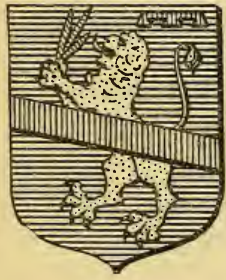

Fig. 200.

Dicesi fermo l'animale posato sulle sue zampe senza che l'una avanzi l'altra: sedente posato sulle zampe posteriori: coricato allorchè l'animale giace, ma colla testa alta, per non confonderlo col dormente (I).

La posa regolare del leone è la rampante (fig. 200).

Si rappresenta il suo corpo svelto, e nel XIV secolo è ritto in modo che combina

(c) Per la definizione dei termini che esprimono gli attributi degli animali, vedi Vocubolario Araldico del Guelí (Manuali Hoepli). 
coll' asse longitudinale dello scudo: le fauci aperte o talora spalancate armate di denti, colla lingua fuori che forma una linea sventolante colla punta ripiegata, colla guardatura feroce.

Nel cinquecento è slanciato, e la sua figura forma quasi una linea serpeggiante; la coda è ripiegata e corre parallela al corpo. Nel secolo XV e dopo, la coda è spesso partita con ambo le parti intrecciate insieme, ciascuna con un ciuffo.

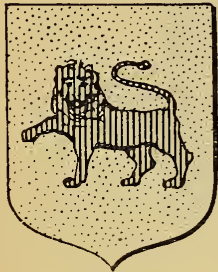

Fig. 201.

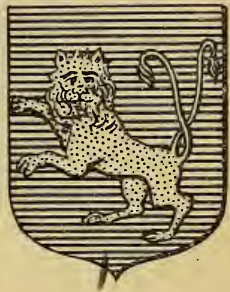

Fig. 202.

La corona di cui sono decorati il leone e gli altri animali dev'essere la semplice corona di elmo, formata di un cerchio con un intero trifoglio nel centro e con un mezzo a ciascuno dei lati, e dev'essere posata perpendicolarmente sopra il capo dell'animale, secondo la linea longitudinale dello scudo.

Il. leopardo, che in araldica non differisce del leone se non per la posizione, si rappresenta passante colla testa sempre di fronte, la coda rivolta sul dorso e ripiegata all' infuori (fig. 20I).

Dicesi illeonito il leopardo colla testa di fronte, ma 
rampante come il leone (fig. 202); mentre all'incontro leopardito un leone passante e colla testa in maestà.

Il cervo può essere corrente, saliente (fig. 203), in riposo, slanciato, ferito, ramoso di smalto diverso, ecc., La sola sua testa in prospetto dicesi rincontro; il suo teschio, massacro, le corna, rami di cervo.

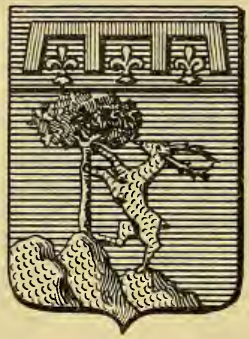

Fig. 203.

Il cinghiale si pone ordinariamente di profilo o passante, e si distingue dal porco per i suoi lunghi denti, appellati nel linguaggio blasonico difesa.

Il camoscio si rappresenta colle quattro zampe riunite e pronte al salto, il capo alzato e in atto di stare all'erta.

La volpe viene per regola rappresentante saltellante; il lupo (fig. 204) passante, rapace, ecc.

Il cane (fig. 205) con orecchie cascanti chiamasi bracco; con orecchie lunghe e rivolte all' insù e col corpo magro, veltro; colle orecchie mozze e collaretto, mastino. 
Questo animale si figura passante, braccante o sedente, ecc.

Il cavallo si pone nello scudo, passante, inalberato, spaventato, assiso, pascente, galoppante, furioso, allegro, gualdrappato, ecc.

Il toro e il bue si figurano, il primo colle corna in forma di mezzaluna, il secondo colle medesime ricurve, entrambi di frequente colla testa abbassata, pronti all' urto.

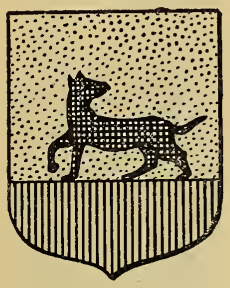

Fig. 204.

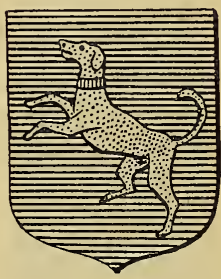

Fig. 205.

L' elefante, colle orecchie intagliate a ventaglio, e colla proboscide ricurva. Si rappresenta nelle arme, fermo, sostenente una torre, armato, gualdrappato, ecc.

Altri quadrupedi frequenti nelle armi sono il daino, la capra, la pecora, l'agnello (detto agnello pasquale quando tiene fra le zampe una croce da cui pende una banderuola crociata), l'asino, l'orso, la tigre, il cammello, la lepre, il coniglio, il gatto, l' istrice, la lince, il tasso, il castoro, la lontra, la scimia, lo scojattolo, la martora, la marmotta, la faina, la donnola, il topo, la talpa, ecc. 
UCCELLI.

Per $\mathrm{i}$ volatili le pose più usitate sono: volante, fermo, sorante (in atto di spiccare il volo), ecc.

L' aquila (che col leone è la figura più nobile del blasone), si rappresenta stilizzata, in atto di salire e di attacco, mostrante il petto e colla testa piegata a destra (a sinistra se è rivolta), gli artigli adunchi e distesi, le ali spiegate, la coda ornamentale e colle penne disposte a ventaglio (fig. 206).

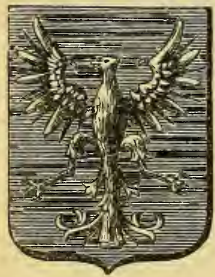

Fig. 206.

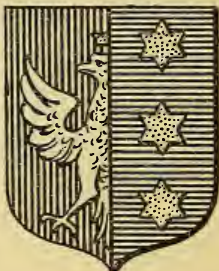

Fig. 207.

L'aquila bicipite ha due teste, di cui una volta a destra e l'altra a sinistra. Quando è nera in campo d'oro, imbeccata, membrata e coronata d'oro, dicesi aquila dell'Impero.

Un'aquila mezzo nascosta da una partizione dicesi nascente o uscente quando se ne vede la parte superiore, movente quando se ne vede un lato (figura 207). Le aquile in numero si chiamano aquilotti (fig. 208); le aquile mutilate, ossia senza becco e senza piedi, alerioni (fig. 209). 
Appariscono negli scudi anche le singole parti dell' aquila, ma più di frequente le ali accoppiate od una sola.

Due ali riunite si chiamano un volo (fig. 2 ro); un'ala sola semivolo (fig. 2I I).

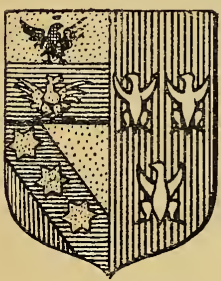

Fiğ. 208.

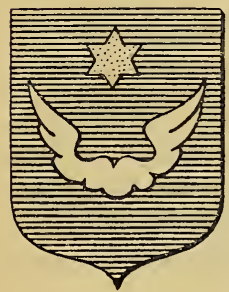

Fig. 210.

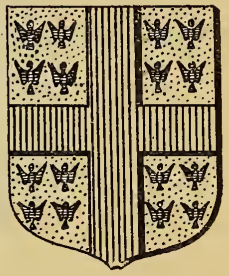

Fig. 209.

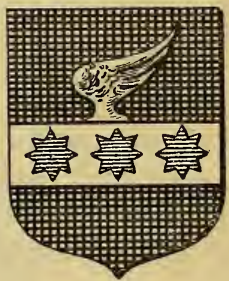

Fig. 211.

Anche le piume sono usatissime, specialmente in cimiero; ma più spesso le piume di struzzo e di pavone.

Oltre l'aquila, hanno un tipo araldico i seguenti volatili: il pellicano, in atto di lacerarsi il petto col becco 
per nutrire i suoi piccini, ossia colla sua pietà; la grù ritta sopra una zampa, tenente nell'altra un sasso (il quale si chiama vigilanza); la cicogna col colo ritirato; il gallo con una zampa alzata; il pavone colla coda a ruota occhiuta; il falcone colla testa incappucciata; la colomba (spesso recante nel becco un ramoscello d'ulivo). Si trovano inoltre fagiani, sparvieri, corvi, astori, beccaccie, pernici, piche, alcioni, aironi,

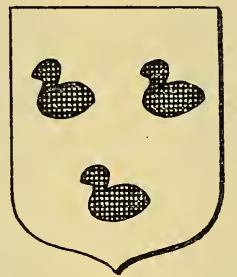

Fig. 212.

avvoltoi, pappagalli, struzzi, oche, ùpupe, civette, gufi, cigni, cormorani, rondini, passere, allodole, ecc.

Diconsi anatrelle le anatre mancanti di becco e di zampe (fig. 212); merlotti, i piccoli uccelli egualmente mutilati.

ALTRI ANIMALI.

I pesci si rappresentano in generale intieramente di uno smalto, e nelle antiche armi le pinne sono dipinte in contrasto.

Si pongono nelle armi addossati, affrontati, curvi, 
montanti, notanti, o in fascia, in banda, in sbarra, in, palo, orecchiuti, scagliosi, pinnati, spasimati, caudati, coronati, ecc.

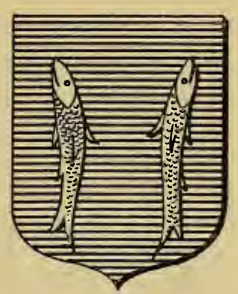

Fig. 213.

I pesci che hanno un tipo araldico sono il delfino, la cui posizione ordinaria nello scudo è di essere curvo in semicerchio col muso e la coda volti verso la destra dello scudo; la trota molto curvata e seminata di

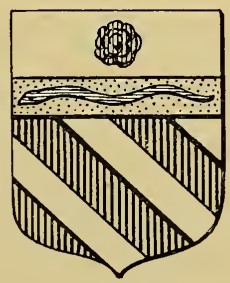

Fig. 214.

punti rossi; i due barbi ritti, salienti, contrapposti (figura 213).

L' anguilla. ¿̀ sempre ondeggiante in fascia (fig. 2I4). 
Il serpente che si rappresenta attortigliato, ondeggiante piegato in giro, o in doppio giro, annodato, alato, coronato, ecc.

Il serpente posto ondeggiante in palo, il capo in fascia, di profilo e volto a destra, prende il nome di $b i$ scia, e se ha in bocca un fanciullo uscente, dicesi ingollante (fig. 215). $\dot{\mathrm{E}}$ celebrè il biscione dei Visconti, ossia " la vipera che i Milanesi accampa ».

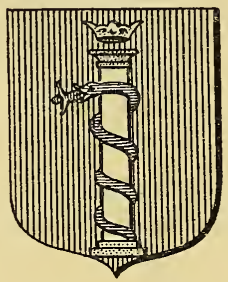

Fig. 215.

Altri animali che si trovano nell'armi sono la lucertola, il ramarro, la salamandra (che si rappresenta fra le fiamme), il coccodrillo, il camaleonte, la rana, la testuggine, il gambero, la lumaca, la conchiglia, l'ape, la farfalla, la cicala, la formica, il grillo, la locusta, lo scorpione, il ragno, la mosca, ecc.

Oltre agli attributi sopra menzionati, gli animali possono blasonarsi affrontati, addossati, armati, afferrati, arditi, baglionati, collarinati, combattenti, contrapassanti, contrarampanti, coricati, cornati, decapitati, divoranti, dismembrati, diademati, diffamati, dragonati, evirati, guardanti, impugnanti, illuminati, in- 
catenati, linguati o lampassati, montanti, marinati, mascherati, nascenti, natanti, orecchiati, pascenti, posati, recisi, sanguinosi, sonagliati, squamosi, serpeggianti, tormentati, unghiati, vestiti, ecc. (I).

\section{VEGETALI.}

I più comuni sono l'alloro, la quercia, la rovere, l'olivo, la palma, il castagno, il ciliegio, il cipresso, il faggio, il fico, il mandorlo, il melo, il moro, il pino,

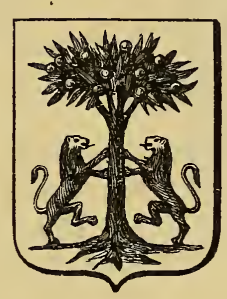

Fig. 216.

il salice, l'olmo, il cedro, il trassino, la felce, il tiglio il melograno, il noce, il pero, il pesco, il pioppo, il platano, la vite, ecc.

Si rappresentano spogliati o secchi, fustati di smalto diverso, fioriti, caricati, diramati, nodosi, mozzi, piantati o terrazzati (posti sopra una piccola zolla di terreno per lo più verde), recisi, fruttiferi, sradicati (figura 216), scoronati, ecc.

(r) Vedi Vocabolario Araldieo del Guelfi (Manuali Hocpli). 
La rovere, nell'araldica italiana, ha generalmente quatro rami passati in doppia croce di Sant'Andrea (fig. 217).

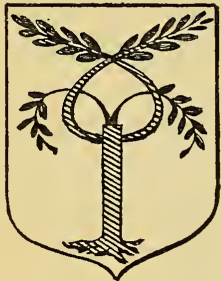

Fig. 217.

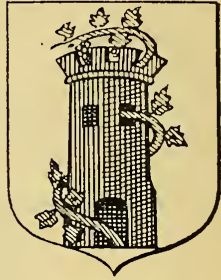

Fig. 218.

La vite si rappresenta pampinosa, e spesso accollata a un albero, a una colonna, a una torre (fig. 2I8). Lo smalto ordinario degli alberi è verde; però si vedono di altri colori e smalti.

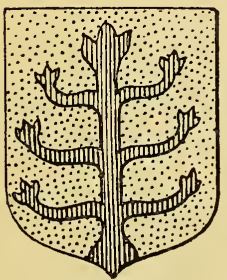

Fig. 219.

Il vepre è una figura araldica che rappresenta un ciliegio selvatico di sette rami. Le alterazioni ne cangiarono la forma primitiva, ed ha preso quella di un candelabro a sette bracci, che sembrano fioriti all'estremità (figura 219).

I fiori ebbero il loro linguaggio cavalleresco e amoroso nei tornei.

Gli attributi loro sono gambuti, fogliati, piantati, legati, seminati, ecc.

Fra essi la rosa e il giglio appariscono nell'araldica 
non già nella forma naturale, ma stilizzati in una figura loro propria.

La rosa si rappresenta allargata di cinque foglie o petali, con un bottone nel centro e senza gambo (figura 220). Talora si figura al naturale.

Il giglio araldico è diverso dal naturale. Esso si rappresenta composto di tre foglie, l'intermedia al disopra e al disotto più appuntata, le laterali superiormente ri-

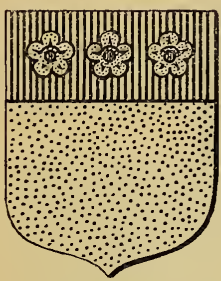

Fig. 220.

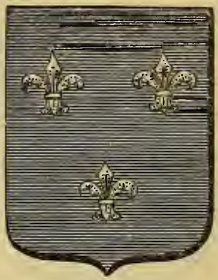

Fig. 221.

piegate e nelle punte inferiori ricurve, tutte e tre legate con un nastro (fig. $22 \mathrm{I}$ ).

Il giglio, in francese fleur de lys, è reputato dagli araldisti il più nobile di tutti i fiori. Svariatissime ha le sue forme araldiche, quasi innumerevoli; si fa rabescato, a ferro di lancia, di francisca, di labarda, a foglie appuntate o ritondate, a più di tre foglie, o forate o merlettate o accartocciate, ecc.

Anticamente il giglio di Francia fu bottonato come quello di Firenze, ma in seguito di tempo spogliò $\mathrm{i}$ suoi ornamenti, e rimase come al presente una figura di tre foglie, arrotondata la media o a guisa di lancia, 
e le due altre incurvate e riunite verso la base da una piccola stanghetta.

I gigli o fiordalisi di Francia furono portati in Italia dal re Carlo VIII nella sua discesa in Italia. Le città di Pisa, di Napoli, di Genova, di Milano aggiunsero le armi di Francia alle loro.

Un campo d'azzurro seminato di gigli d'oro dicesi seminato di Francia (fig. 222).

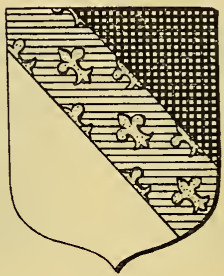

Fig. 222.

Il capo d'azzurro coricato di tre gigli d'oro e di un lambello di rosso chiamasi capo d'Angiò (fig. 203).

Il giglio fiorentino pretendono molti araldisti fosse una concessione di Carlo Magno, modificato col correre del tempo coll'aggiunta dei bottoni e dei boccioli.

Il Crollalanza confuta questa opinione osservando, essere dubbio che Carlo Magno avesse dei gigli per insegna. Egli suppone che il nome stesso di Fiorenza abbia suggerito di prendere per emblema della città un fiore, e si scegliesse il fiore più frequente delle sue convalli, il gaggiolo.

Il giglio di Firenze si dipinse bianco in campo rosso; 
ma quando i ghibellini furono cacciati fuori della città dai guelfi, nel I25 I, si alternarono gli smalti, come ne fa testimonianza Dante nel canto XIV del Paradiso :

Con queste genti vid'io glorioso

E giusto il popol suo tanto, che 'I giglio Non era ad asta mai posto o a ritroso Nè per division fatto vermiglio.

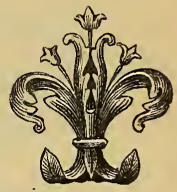

Fig. 223.

Il giglio di Firenze è aperto o allargato, o bottonato, o bocciolato (fig. 223).

Fra le foglie sono da notarsi il trifoglio (fig. 224),

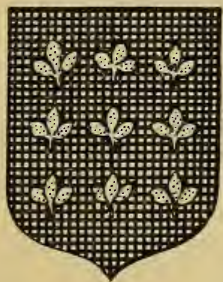

Fif 224.

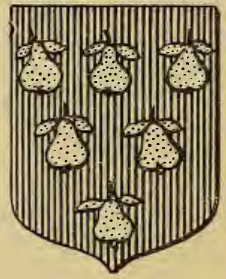

Fig. 22 .

che spesso è stilizzato, la terzafoglia (rosa a tre petali), la quartafoglia (rosa a quattro petali), la quintafoglia (rosa a cinque petali senza bottone), la foglia d'edera, la foglia di tiglio, la foglia d'ortica e la foglia marina. Queste due ultime hanno una forma convenzionale nell'araldica tedesca e inglese.

I frutti più comuni sono la melagranata, il grappolo d'uva, la ghianda, la pigna, la pera (fig. 225), la 
mela, l'oliva, la castagna, la noce, la nocciuola, la fragola, la ciliegia, la zucca, la rapa, la spiga, ecc.

CORPI CELESTI, MONTI, FIUMI, ECC.

Il Sole viene rappresentato come un disco nel quale è la figura di una faccia umana, contornata di sedici

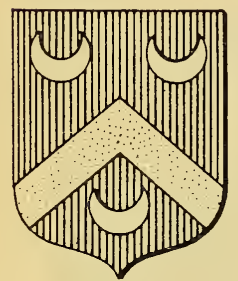

Fig. 226. raggi fiammeggianti, di smalto dorato.

Se il disco è vuoto, chiamasi sole rozzo o ombra di sole; se sta nell' angolo superiore destro, levante o orizzontale a destra; nell'angolo superiore sinistro, $\operatorname{tra}$ montante o orizzontale a sinistra. La Luna, dicesi luna quando è raffigurata piena, il che è raro; crescente quando è scema, ossia la mezzaluna, che ordinariamente è montante, cioè colle corna rivolte verso il capo (fig. 226), posizione che non è necessario blasonare, oppure volta a destra (figura 227), rivolta a sinistra, rovesciata (fig. 228), due addossate (fig. 229), tre addossate in cuore (fig. 230).

Le stelle sono forse la figura più comune negli stemmi. Si fanno di 5, di 6 o di 8 raggi; raramente di 16. Il numero dei raggi deve essere blasonato. Alla fig. 23 I stella di 6 raggi, alla fig. 232 stella di otto raggi.

La cometa dev'essere munita di coda, e impropriamente si blasona come cometa la stella di r 6 raggi; si pone ondeggiante, crinita, orizzontale a destra od a 


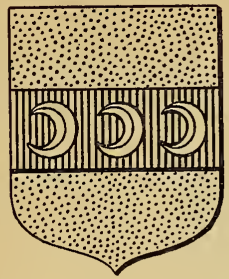

Fig. 227.

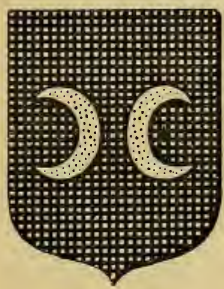

Fig. 229.

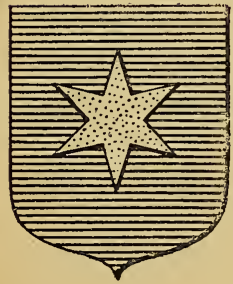

Fig. 231.

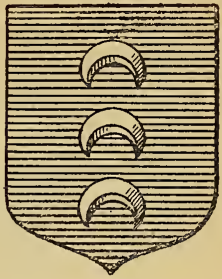

Fig. 228.

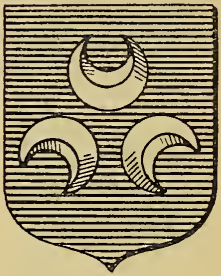

Fig. 230.

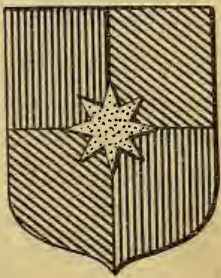

Fig. 232. 
sinistra, in fascia, in sbarra, in banda, in palo, cadente, caricata, ecc. (fig. 233).

L'arcobaleno è una fascia o una banda centrata, divisa di rosso, oro ed azzurro, oppure di altri colori (fig. 234).

Le nuvole si coloriscono di azzurro o di argento, e servono anche di sostegno a braccia o mani armate o benedicenti.

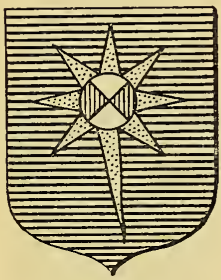

Fig. 233.

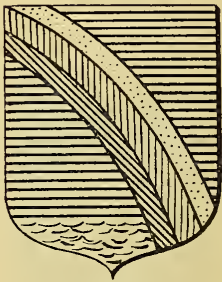

Fig. 234.

Il fulmine è figurato a raggi fiammeggianti.

Il fuoco è rappresentato con fiammelle guizzanti; i venti sotto la forma di teste soffianti.

Il mare occupa il basso dello scudo, è d'azzurro o d'argento, e si blasona calmo, agitato, fluttuoso.

I fiumi si rappresentano come fascie ondeggianti di azzurro o di argento.

Gli scogli, secondo il Ginanni, devono sempre sorgere dalle onde.

Le rupi hanno la forma dei coni appuntati e dentellati.

I monti, si rappresentano formati di 2030 più 
monticelli, mentre 1a montagna è figurata al naturale di un sol pezzo e movente dalla punta.

La disposizione dei monti a pilastrini (simili a quelli che a piramide figurano il calvario sotto la croce) è

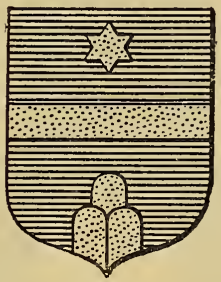

Fig. 235.

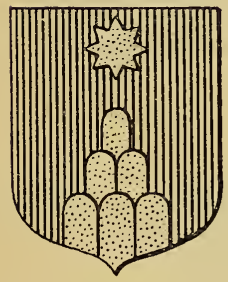

Fig. 236.

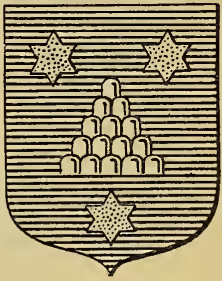

Fig. 237.

speciale all'araldica italiana; e si blasona al monte di $3,5,6$, I0, is cime, ecc. (fig. 235, 236 e 237).

FIGURE ARTIFICIALI.

Le arti e i mestieri forniscono figure al blasone. Così fu fatto nell' araldica antica; ma in quanto ai moderni 
oggetti, raccomandano gli araldisti, che per conservare all'arte quel carattere antico che è proprio dello scudo e dell'elmo, non si pongano nel primo degli oggetti i quali stiano quasi in contradizione con quelle armi cavalleresche.

Non si possono stabilire delle regole in proposito; guida a far bene il gusto artistico.

Varie sono le divisioni di queste figure.

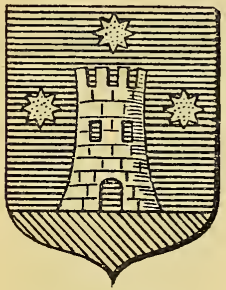

. Fig. 238.

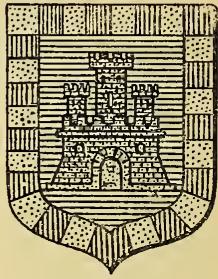

Fig. 239.

Alcuni araldisti le distinguono in quattro classi: I. ${ }^{\circ}$ fabbricati; $2.0^{\circ}$ istrumenti e suppellettili; $3 .^{\circ}$ vesti; $4^{\circ}$ armi.

Il Crollalanza le classifica a seconda della loro origine e delle cause che le hanno, in generale, introdotte nel blasone.

Nomineremo le figure artificiali più frequenti nelle armi.

La torre (fig. 238) e il castello (fig. 239) si vedono in un gran numero di stemmi, e prendono forme variatissime; gli attributi che si blasonano sono merlato, finestrato, aperto, chiuso, torricellato, murato. Il merlato 
alla guelfa ha i merli quadrati; alla ghibellina, i merli ritagliati internamente a triangolo.

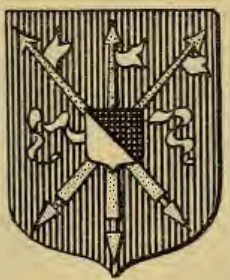

Fig. 240.

Altri fabbricati sono le case, le chiese, i ponti, i pozzi, i molini a vento, le piramidi, le fornaci, ecc.

Fra le armi, armature e figure guerresche primeggiano la spada, la lancia (fig. 240), la scure, l'ascia

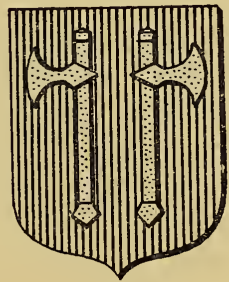

Fig. 211.

d'armi (fig. 24I), la mazza, la picca, la balestra, la freccia, il bolzone, il martello d'armi, l'alabarda, l'ar- 
chibugio, il cannone, la granata, la corazza, lo sperone, le rotelle di sperone (fig. 242), l'arpione, la scala ossidionale, le catene da barriera (fig. 243), la saraci-

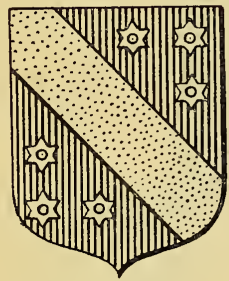

Fig. 242.

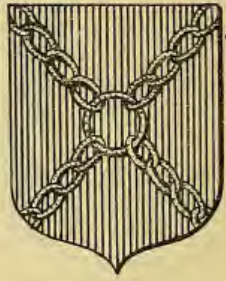

Fig. 243.

nesca, la quintana, la palizzata, l'ariete ossidionale, il tribolo (fig. 244), ecc.

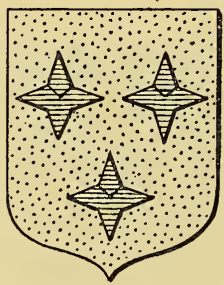

Fig. 244.

Fra le figure tratte da dignità, cariche, giurisdizioni, si notano la corona, lo scettro, il globo imperiale, la tiara, la mitra, il pastorale, il bastone di comando, il 
caduceo, il fascio consolare, la mano di giustizia, il gonfalone, la bandiera, la chiave (fig. 245), ecc.

Altrove vedonsi aratri, erpici, gioghi, ruote, ruote di molino, ferri di molino, ferri da cavallo, corni di caccia, trombe, arpe, barche, navi, ancore, calici, candelabri, chiodi, bordoni da pellegrino, campane, torcie, libri, compassi, martelli, ancudini, zappe, vasi, caldaie

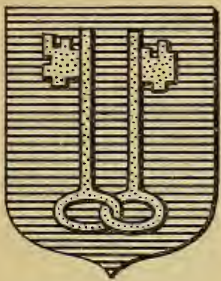

Fig. 245.

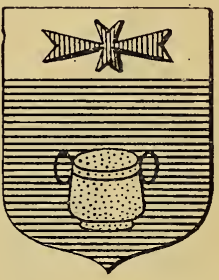

Fig. 246.

(fig. 246), lampade, clessidre, specchi, barili, bilancie, sonagli, dadi, rocchi di scacchiere, anelli, guanti, fermagli, cappelli, cappucci, calzari, ecc.

Gli attributi propri di alcune di queste figure sono: acceso, affibbiato, affustato, attaccato, banderuolato, battagliato, cerchiato, coperto, cordato, fluttuante, gomenato, guarnito, guernito, imboccato, impugnato, incoccato, infiammato, infilzato, manicato, tegolato, terrazzato ed altri meno usati (I).

(I) Vedi Vocabolario Araldico del Guelfi (Manuali Hoepli). 
FIGURE CHIMERICHE.

Le favole dai crociati riportate dall'Oriente, le tradizioni pagane, i simboli (come il leone della repubblica di Venezia) diedero luogo nel blasone a diverse figure fantastiche, che quasi tutte ebbero un tipo araldico speciale.

Le più comuni furono: il drago, il grifo o grifone, la pantera, il liocorno, l'aquila bicipite, la sirena, il centauro, l'arpia e la fenice.

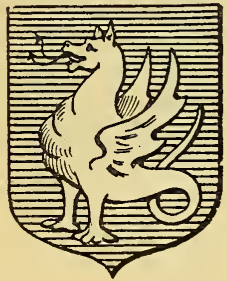

Fig. 247.

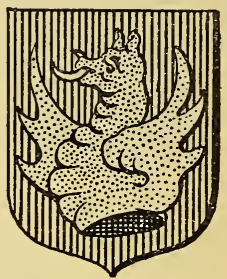

Fig. 248.

Il drago è rappresentato come un rettile alato col capo aguzzo e lc fauci spalancate, dalle quali escono fiamme, la lingua sporgente e vibrante talora terminata in punta di lancia; le zampe leonine o di aquila, la coda grossa e inanellata (fig. 247). Quando se ne vede soltanto la metà anteriore dicesi reciso (fig. 248).

Il grifo ha il petto e il capo dell'aquila, il corpo di leone, collo spiumato, le ali spiegate, artigli spalancati; il ventre e le zampe posteriori e la coda sono di leone. Si raffigura però anche in altre maniere a seconda dello 
stile, delle consuetudini araldiche dei diversi paesi, del capriccio degli artisti (fig. 249).

La pantera non assomiglia alla naturale, ma è un composto di leone, di aquila e di drago; cioè il capo di drago, il corpo di leone e nelle zampe anteriori schiude gli artigli dell'aquila, e la coda leonina.

Il liocorno è un cavallo saltellante o seduto, con unghie fesse, zampe pelose, coda leonina, e con un lungo corno attorcigliato in fronte.

La sirena è una figura di donna nuda che dalla cintura in giù termina in un pesce di una o due code, ch'essa tiene di solito con ambo le mani. Quando è posta entro un tino chiamasi Melusina.

Il centauro è metà uomo e metà cavallo, corrente, colla testa e il dorso rivolto, e spesso saet-

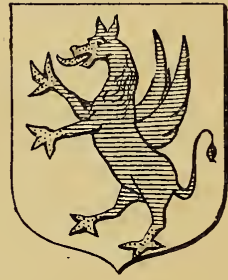

Fig. 249. tante.

L'arpia è una figura con volto e petto femmineo, corpo, ali, artigli e corpo d'avvoltoio, e orecchie d'orso.

$\mathrm{La}$ fenice, uccello favoloso, e nel quale gli antichi imaginarono che quando era presso a morire si costruiva una pira dincenso e di mirra, vi dava fuoco e vi si ardeva dentro; e che dalle sue ceneri nascesse un verme che rivestito di penne, diventava la rediviva fenice.

Nello scudo si figura di profilo, colle ali semispiegate, sopra un rogo, che si chiama immortalita, e che non si blasona se è dello stesso. La fenice di rado è rolante e per lo più si vede nello scudo riguardante 
un sole posto nel primo cantone simboleggiante la gloria.

La sfinge ha volto e busto di donna, corpo di cane, zampe di leone e coda di drago.

La chimera ha testa di donna, petto e zampe posteriori d'aquila, zampe anteriori di leone, e coda di serpente.

L'idra è un serpente a sette teste.

L'anfittero è un serpente alato, di cui la coda ravvolta in spire termina in un'altra testa e spesso in parecchie.

Il basilisco è un rettile a corpo squamoso, coda da serpente, testa di gallo, di falcone o di drago, con due, quattro o otto zampe da uccello.

L'ircogallo è un gallo colla testa di capro.

Trovansi inoltre dei leoni e altri animali dragonati, ossia desinenti in dragone, marinati, ossia desinenti in coda di pesce, pavonati con coda di pavone, alati, androcefali, ornitocefali, monocefali (se con una sola testa comune a due corpi), ecc. Nell'arma della famiglia Faà si vede una fata mostruosa che ha la metà superiore di donna ignuda e scapigliata, le ali di pipistrello, le gambe di uccello palmipede e la coda di serpe finiente in saetta.

Molte altre imagini fantastiche si osservano ancora, specialmente nel blasone straniero, che ci asterremo dal descrivere. 


\section{CAPITOLO IX.}

\section{Elmo, Cimiero e Svolazzi.}

L'elmo costituisce una parte integrante esterna dell'arme, perchè quantunque nel XII secolo lo scudo soltanto abbia costituito l'arme, in appresso, e quando fiori l'arte araldica, divenne il segnale del cavaliere nelle giostre e nei tornei.

Per la qualcosa l'elmo indicó i caralieri e le case nobili, nè fu concesso di cimarne lo scudo nè agli ecclesiastici, nè alle donne, per quanto dame; neppure si die' alle città, ai comuni, alle corporazioni, se non che per concessione.

La Consulta Araldica del Regno concesse l'uso dell'elmo anche alle persone che, non essendo nobili, abbiano diritto di portare uno stemma gentilizio.

Variarono le forme degli elmi a seconda dei tempi e dei diversi armamenti delle milizie.

Gli elmi per cosi dire più araldici sono di tre forme diverse.

I. L'elmo tinare o pentolare del XIII e XIV secolo.

2. L'elmo chiuso.

3. L'elmo graticolato. 
Il primo è fabbricato di due o quattro piastre, e di una che lo coperchia.

L'apertura per gli occhi consiste in due tagli longitudinali, o di una fenditura aperta (taglio visuale) tra la parte superiore ed inferiore. Dai lati trovansi alcuni piccoli fori, perchè vi circolasse l'aria, ed un intaglio crociforme, che serviva a fissare l'elmo nell'anello della corazza.

Il secondo, l'elmo chiuso, ha una forma tinale curva, elegante; la parte superiore serra la rotondità della testa, la inferiore sporge nel mezzo in fuori e forma di profilo una punta. Frammezzo ha il taglio visuale; arrivava in giù sul petto e sul dorso, dove era affibbiato.

Il terzo, l'elmo graticolato, è rotondato di sopra e davanti; il taglio visuale molto aperto è difeso da 50 7 affibbiature.

Gli elmi pentolari e tinali appartengono ai secoli XIII e XIV; gli elmi chiusi al XV e XVI secolo; gli elmi graticolati ai tempi successivi.

Gli elmi tinali si trovano posti generalmente sopra gli scudi triangolari; i chiusi sugli scudi triangolari, ma in specie sulle targhe: i graticolati sugli scudi semirotondi o alemanni.

L'elmo non deve occupare maggior larghezza del $5 / 7$ del lato superiore dello scudo.

Agli elmi nobili suol porsi ad ornamento una catenella o nastro in forma di moneta o di rosetta.

Il posto ordinario dell'elmo è sopra la metà superiore dello scudo. Nello scudo inclinato vien posto sopra l'angolo più alto.

Per regola generale si pone un solo elmo, sopra un 
solo scudo; ma siccome si mettono più armi in un solo scudo, in questo caso per ciascuna delle medesime può porsi l'elmo corrispondente, come si usa specialmente in Germania.

Quando più elmi cimano lo scudo si pone a destra quello appartenente all'arma principale; essendovene tre il principale sta nel mezzo; allorchè sono in maggior numero, l'estremo a destra e l'estremo a sinistra sono gli ultimi.

Quanto alla posizione dell'elmo quella ordinaria, negli scudi diritti, è di faccia, negli scudi inclinati, è posto in profilo verso il lato della pendenza dello scudo.

Due elmi sopra uno scudo volgono l'un contro l'altro.

Se sono più di due in numero pari, una metà è volta a destra, l'altra a sinistra; se dispari, quello del mezzo guarda in avanti, e gli altri d'ambo i lati sono rivolti verso il medesimo di profilo.

Nel secolo XVI invalse la pratica che l'elmo graticolato (dei tornei) appartenesse ai nobili, e l'elmo chiuso alle armi cittadinesche.

Però in questo stesso secolo l'araldica francese e inglese, ed anche l'italiana, si allontanò dalle regole scientifiche dell'arte, conservate dalla Germania, e ne stabili delle altre sulla forma e posizione degli elmi a seconda dei gradi e dei titoli della nobiltà.

Per gli elmi e le corone di grado ci serviremo delle parole stesse della deliberazione della Consulta Araldica del Regno, che stabilisce quali devono essere gli ornamenti esteriori delle armi.

Poche sono le differenze che passano dalle regole che ha essa fissate, e quelle dell'arte araldica francese ed inglese.

Crollalanza. 
Gli araldisti della Germania, fedeli alle antiche tradizioni cavalleresche, hanno conservato gli elmi medioevali, nè riconoscono il sistema moderno.

Gli imperatori e i re portano l'elmo di oro rabescato ed aperto intieramente, posto di fronte sopra lo scudo.

I principi e duchi egualmente di oro, rabescato $\mathrm{e}$ semi aperto, colla visiera alzata metà, e colla gorgieretta dello stesso (fig. 250).

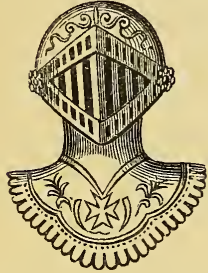

Fig. 250.

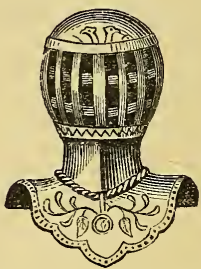

Fig. 251.

L'elmo di marchese è di argento, rabescato e semi aperto, colla visiera alzata metà, graticolato di I i pezzi d'oro, e colla gorgieretta dello stesso (fig. 25 I).

L'elmo di conte è d'argento, rabescato dello stesso, bordato d'oro, posto per un terzo in profilo verso destra, graticolato di diciassette pezzi d'oro, colla gorgieretta dello stesso (fig. 252).

L'elmo di visconte è simile a quello di conte, se non che è in profilo per due terzi ed è graticolato di tredici pezzi.

L'elmo di barone è liscio, bordato d'oro, posto per 
due terzi in profilo verso destra, graticolato di tredici pezzi d'oro, colla gorgieretta dello stesso.

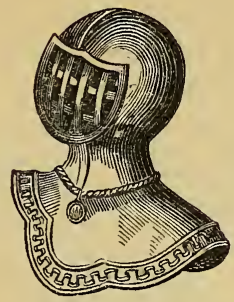

Fig. 252.

L'elmo di patrizio è d'argento, rabescato di oro, posto in profilo verso destra, colla visiera d'oro, alzata a metà (fig. 253).

L'elmo di nobile è d'argento, liscio, bordato d'oro,

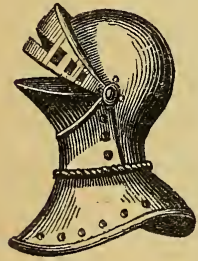

Fig. 253.

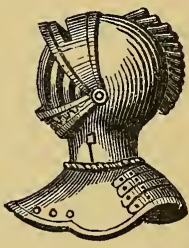

Fig. 254.

posto in profilo verso destra, graticolato di nove pezzi d'oro (fig. 254).

L'elmo delle personé insignite del cavalierato tra- 
smissibile è come quello di nobile, ma graticolato di tre pezzi.

L'elmo delle persone, che non essendo nobili, abbiano diritto di portare uno stemma gentilizio, è d'acciaio liscio, chiuso, posto in profilo pieno verso destra.

Nell'araldica antica vi era anche l'elmo di bastardo, che era di acciaio liscio, intieramente chiuso senza affibbiature e rivoltato, cioè vol to alla sinistra dello scudo (fig. 255).

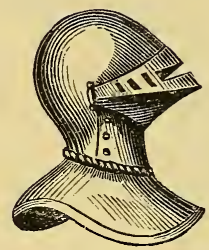

Fig. 255.

I lambrecchini o svolazzi furono primitivamente un riparo all'elmo onde preservarlo dall'umidità dell'aria e dai raggi scottanti del sole. Alcuni araldisti suppongono che simbolizzino i rami di foglie posti dai cavalieri sull'elmo, segnali di vittoria. Altri che dessi siano cosi -frastagliati per i colpi che ricevevano i cavalieri nelle giostre e nelle battaglie.

I lambrecchini debbono armonizzare nello stile colla forma dell'elmo; cosi per esempio all'elmo pentolare conviene una specie di mantellina o pezzo di panno piano ai lembi, ovvero tagliuzzato (fig. 256), sull'elmo chiuso o graticolato si adatteranno meglio i lambrec- 


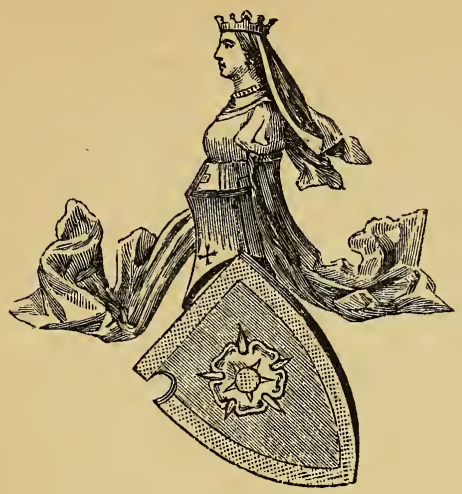

Fig. 256.

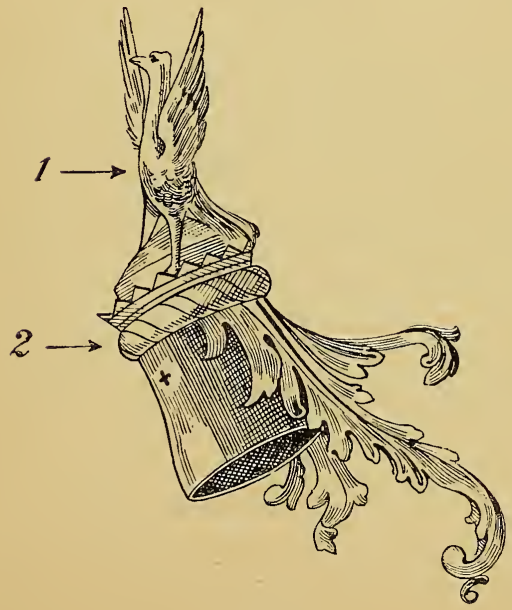

Fig. 257. 
chini a svolazzo, ossia gli ornamentali, proprî dell'arte di quei tempi.

Il cercine è un rotoletto di stoffa colorato degli smalti dello scudo e che teneva fermo sull'elmo il cimiero e i lambrecchini, i quali rappresentano dei pezzi di stoffa, frastagliati a guisa di foglie circondanti l'elmo e pendenti dall'una e dall'altra parte dello scudo.

Nella fig. 257 l'elmo è ornato di cercine e di svolazzi, e sormontato da un cimiero.

Il cimiero è una figura che cima l'elmo.

I cimieri vennero in uso primitivamente per far apparire più alta la statura del cavaliere e per ispirare terrore al nemico colle figure di mostri o di animali feroci; in appresso servirono a distinguere o le fazioni, o i rami diversi di una, o qualche impresa illustre del cavaliere.

Le figure dei cimieri non sono sempre le stesse dello scudo, spesso rappresentano penne, corna, voli, berettoni, bandiere, animali, corpi e membra umane, figure naturali e artificiali, ecc. I cimieri tedeschi sono il più sovente stranissimi.

Nell'araldica alemanna e inglese importantissimo è lo studio dei cimieri, perchè i Tedeschi sono soliti timbrare con più elmi lo scudo; e gli Inglesi hanno per regola che qualunque arma sia timbrata, non dall'elmo, ma dal solo cercine sul quale si alza il cimiero. 


\section{CAPITOLO X.}

\section{DELlE CORONE ED ALTRI CONTRASSEGNI DI DIGNITÀ.}

Le corone poste sopra lo scudo, servono a distinguere la dignità del proprietario dell'arma.

Esse si debbono distinguere, secondo il Sacken, dalle corone degli elmi le quali servono a congiungere l'elmo col cimiero, e dalle corone che servono solo di fregio alle figure dello scudo.

Per altro, nell'araldica italiana, la corona che cima l'elmo è pur quella stessa del grado nobilesco. Il sopralodato araldista non ammette la riunione dell'elmo colla corona di grado; nè che da essa esca il cimiero, proprio dell'elmo.

Il posto delle corone di grado è sopra il margine superiore dello scudo, e quando si pone la corona, insegna il Sacken, non si deve porre l'elmo.

Ciò non toglie che nell'uso approvato non si pongano regolarmente le corone volanti sugli scudi; come pure si decorino gli elmi delle corone di grado o dignità, incominciando da quella pontificia. Alcune di 
esse caddero cogli Stati sovrani, dei quali decoravano il principe, come il corno dogale di Venezia, e la corona granducale toscana.

Queste diverse corone possono vedersi nell'Arte del Blasone del Ginanni.

A questo saggio elementare basterà indicare alcune delle principali corone tanto sovrane che nobiliari, attenendoci per queste ultime al Regolamento della Consulta Araldica, tanto più che la Consulta non ha portato cangiamento sostanziale alla forma delle corone di grado, se ne eccettui quelle di patrizio, di cavaliere, e di nobile.

Nell'ordine ecclesiastico, scrive il dotto monsignor Barbier de Montault, gli stemmi non esprimono un segno di nobiltà; essi indicano una dignità o carica ecclesiastica, di guisa che qualunque dignitario nobile o no, unicamente per il grado del suo ministerio, ha il diritto e il dovere, di assumere delle armi personali, se non ha la propria gentilizia (I). Gli ecclesiastici timbrano i loro scudi colla tiara, colle mitre, e coi cappelli.

La forma della tiara è ovale, ed è composta di una berretta o callotta di seta bianca o di stoffa di argento, cinta di tre corone, e sormontata di un globo ornato di una croce.

La tiara è posta di fronte sull'orlo superiore dello scudo, che è accollato sulle due chiavi pontificie situate in croce di Sant'Andrea, l'una di oro e l'altra di argento, legate di azzurro, caricate di crocette nere, e la triplice croce in palo (fig. 258).

(I) Les armoiries ecclésiastiques d'après le droit commun, 
I cardinali timbrano il loro scudo con un cappello rosso, guarnito di cordoni di seta rossa, intrecciati a losanghe con cinque file di nappe, quindici da ciascuna parte, e posano una croce in palo dietro lo scudo ( $\mathrm{fi}_{1}$ gura 259).

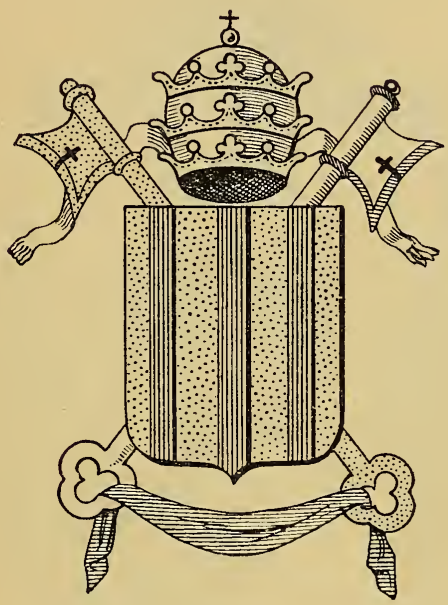

Fig. 258.

Gli arcivescovi primati timbrano lo scudo di cappello verde con quattro file di nappe e la croce semplice in palo dietro lo scudo.

I rescovi timbrano lo scudo della mitra posta di fronte e del pastorale.

Gli abati e i pronotarî della chiesa portano la mitra 
e il pastorale sotto il cappello, che è nero con due nappe.

Le badesse portano lo scudo a losanga come le donzelle, circondato di un rosario nero, e il pastorale

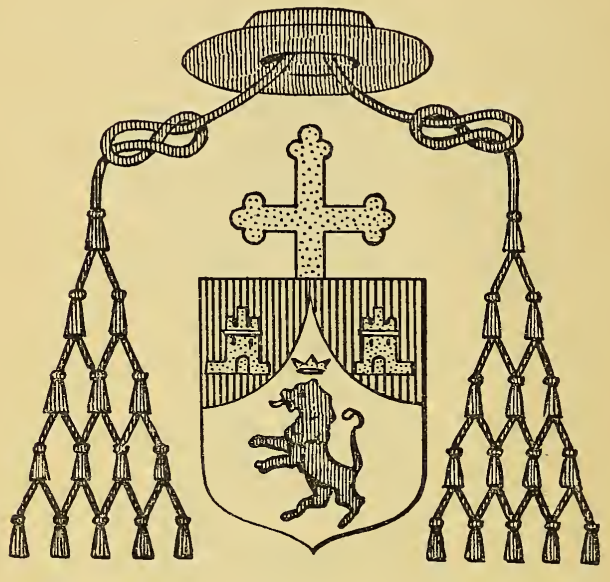

Fig. 259.

in palo dietro lo scudo, rivolto a sinistra come gli abati (I).

La corona del Sacro Romano Impero è una specic di mitra alla foggia persiana, ornata di un globo d'oro sormontato di una croce dello stesso (fig. 260).

(r) Per altri contrassegni delle dignità vedi Appendiçe in Voçabolario Araldico del Guelfi. (Manuali Hoepli). 
La corona reale di Francia è un cerchio d'oro, adorno di pietre preziose e cinta di fiordalisi di oro (fig. 26I). La corona reale è composta di un cerchio d'oro rabescato ed ornato di gemme a varî colori, bruniti ai

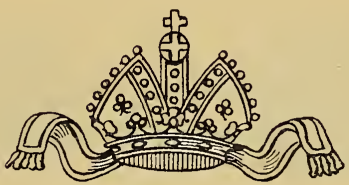

Fig. 260.

margini; sostenente sedici basse punte cimate da perle, metà delle quali, alternativamente, formano il cuore di altrettanti fioroni, ognuno da tre foglie doppio composto, e sormontato da ugual numero di semicircoli o diademi contornati di perle, riunite superiormente sotto

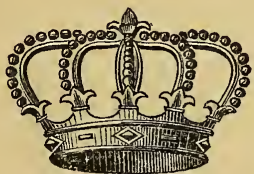

Fig 261.

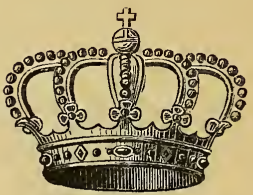

Fig. 262

un globo di azzurro fasciato, centrato, e timbrato da croce trifogliata, il tutto d'oro; la corona stessa cinge internamente un berretto di velluto rosso, e nei disegni, mostra tre fiori intieri nel mezzo, due metà di essi ai lati, quattro basse punte e cinque diademi (fig. 262). 
La corona di principe è un cerchio d'oro tempestato di gemme di vari colori, bruniti ai margini, sostenente nove fioroni d'oro ( 5 visibili) caricati ciascuno di una perla nel cuore; essa corona cinge la base di un tôcco

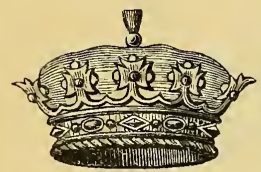

Fig. 263.

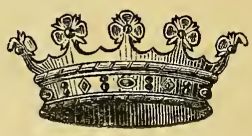

Fig 264.

di velluto color di porpora, sormontato da un fiocco d'oro a pennello (fig. 263).

La corona di duca è uguale a quella di principe, ma senza il tôcco (fig. 264).

La corona di marchese è un cerchio d'oro, come quello della corona di principe, sostenente quattro fioroni d'oro (3 visibili) alternati con dodici perle poste

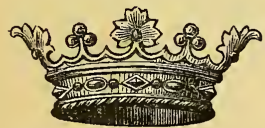

Fig. 265.

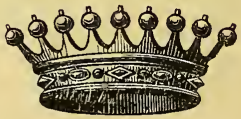

Fig. 266.

tre a tre, piramidalmente, cioè una su due, $€$ sorrette da un piccolo gambo simile a quello dei fioroni (fig. 265).

La corona di conte è un cerchio d'oro rabescato a fogliami smaltati di vari colori, bruniti ai margini $e$ sostenenti sedici grosse perle di cui 9 visibili (fig. 266). 
La corona di visconte è uguale a quella di conte, se non che sul cerchio ha 8 perle soltanto, quattro grosse e quattro piccole alternate (fig. 267).

La corona di barone è un cerchio d'oro, come quello

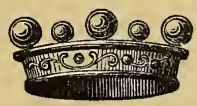

Fig. 267.

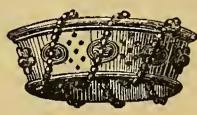

Fig. 268.

della corona dei conti, intorno al quale, sono attorcigliate in banda, ed in sei giri, perle infilzate a guisa di monile (fig. 268).

La corona di patrizio è un cerchio d'oro liscio, brunito al margine inferiore, e sormontato da quattro punte di lancia, alternate con quattro globetti simili a perle, moventi da altrettante basse punte, il tutto d'oro (figura 269).

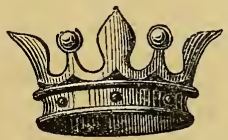

Fig. 269.

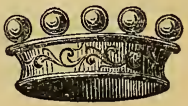

Fig. 270.

La corona di nobile è un cerchio d'oro, puro, velato, rabescato, brunito ai margini, sostenente otto grosse perle ( 5 visibili) posate sul cerchio (fig. 270).

Per la situazione orizzontale delle corone sugli scudi non si disegna sulle figure dipinte e scolpite in basso 
rilievo tutto il numero di fioroni, e di perle sovraindicate, supponendosene poco meno della metà coperto dai fioroni e dalle perle che si presentano di fronte; epperciò vengono disegnati soli 5 fioroni, di cui i due ultimi laterali in profilo, sulla corona di principe e duca: tre soli fioroni, i laterali in profilo e due piramidi di perle su quella di marchese: nove perle su quella di conte; tre grosse perle e due piccole in mezzo ad esse su quella di visconte; tre giri di perle nella corona di barone; cinque perle nella corona di nobile; tre perle su quella delle persone cui compete il cavalierato gentilizio.

La Consulta Araldica, della quale abbiamo riferito le regole intorno alle corone araldiche, non ha portato varianti alle antiche leggi blasoniche, se non che nelle corone di patrizio, di nobile e di cavaliere ereditario.

Quella di patrizio è affatto nuova nel blasone.

Le famiglie di nobiltà generosa in Italia usavano un cerchio d'oro rialzato di quattro punte sormontate da fioroni, con quattro perle, ed anche senza, sovrapposte ad ugual numero di basse punte d'oro.

Presso a poco adoperavasi la stessa corona dai nobili, e in conseguenza da coloro che erano insigniti del cavalierato trasmissibile, per ottenere il quale erano

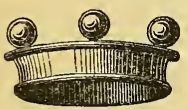

Fig. 271. necessarie le provanze di nobiltà.

$\mathrm{Ci}$ pare nuova anche quella del cavalierato ereditario (fig. 27I); nè ci dispiace che ne sia stata fatta una speciale per il patriziato (di cui abbiamo già data l'incisione), se si pone mente alla importanza storica che i patrizi ebbero nelle repubbliche italiane, dove essi governavano la 
cosa pubblica, animati da uno spirito molto diverso dal feudale.

Siccome l'imperatore Napoleone I fu anche re d'Italia, accenneremo che l'Araldica Napoleonica fu estesa ancora in Italia.

L'imperatore sostitui alle corone e agli elmi una berretta o tôcco di velluto nero, rialzata di vajo, di

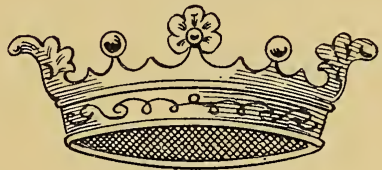

Fig. 272.

ermellino o contro ermellino, secondo il grado del personaggio, e sormontata di piume bianche o d'argento.

Le famiglie di nobiltà generosa in Italia e coloro che erano insigniti della nobiltà equestre del Sacro Romano Impero usavano e usano tuttora una corona quasi simile a quella di marchese, tranne che le i2 perle sono sostituite da 4 soltanto, una su ciascuna punta alternante coi fioroni (fig. 272). 


\section{CAPITOLO XI.}

\section{ORNAMENTI ESTERNI DELLO SCUDO.}

\section{TENENTI E SUPPORTI.}

Sono essi i sostegni dello scudo posti da ciascun lato del medesimo come per sorreggerlo. Diconsi tenenti se rappresentano figure umane; supporti se di animali. Talora lo scudo è sorretto da un solo tenente.

Svariatissime sono le figure di uomini, di donne, di animali e di figure ideali che possono servire come tenenti o supporti.

Il citato decreto della Consulta Araldica dispone che i tenenti e i sostegni non si accorderanno fuori che alle persone aventi diritto all'uso del manto sulle armi, ai marchesi e ai conti, escludendo sempre i sostegni usati dal Re.

\section{PADIGLIONE E MANTO.}

Il Bouton crede che dai lambrecchini sia provenuto l'uso dei manti che ornano le armi dei principi e dei duchi, e i padiglioni degli imperatori e dei re. 
L'uso dei tornei, nei quali si esponevano su tappeti preziosi le armi dei combattenti, i padiglioni e le tende ove i capi delle quadriglie attendevano l'ora di entrare in lizza, furono l'origine dei padiglioni e dei manti.

Per la nuova araldica italiana il distintivo del manto è riservato, oltre al Re e alla Regina, ai Principi ed alle Principesse Reali, ai cavalieri dell'Ordine supremo dell'Annunziata, ai Grandi Uffiziali dello Stato, ai Principi ed ai Duchi.

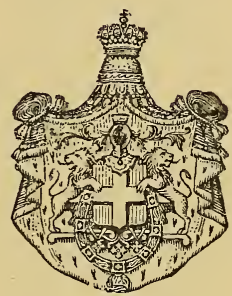

Fig. 273.

Il manto del Re, della Regina, dei Principi e delle Principesse Reali, e dei Principi e delle Principesse del sangue, è di velluto porpora soppannato di armellino frangiato d'oro, la frangia attaccata ad un gallone alternativamente composto di croci scorciate e di nodi di Savoja (fig. 273).

Il padiglione del re è sparso esteriormente di crocette d'argento e di nodi d'oro di Savoja, alternati con rose ế lingue di fuoco d'oro.

Il manto dei cavalieri dell'Ordine Supremo è di vel- 
luto azzurro soppannato di seta bianca, bordato d'oro, la bordatura caricata di nodi di Savoja d'oro.

Il manto dei Grandi Uffiziali dello Stato, dei Principi e dei Duchi è di velluto di porpora, soppannato di seta bianca e bordato d'oro.

Gli elmi, dai quali muovono i manti, si pongono sempre di fronte.

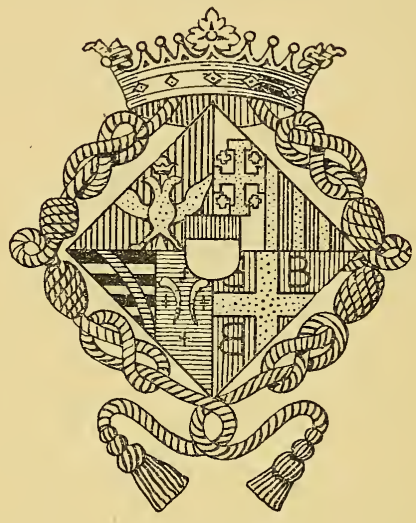

Fig. 274.

LA CORDELLIERA E IL LACCIO D'AMORE.

Il Ginanni definiva la cordelliera (fig. 274) come una specie di collana che a guisa di laccio d'amore è formata da due cordoni moventi dalla corona attortigliati intorno allo scudo fatta a losanga, svolazzanti infine e fioccati. 
Il laccio d'amore ebbe origine dai nastri che i cavalieri ricevevano dalle loro dame come favori nei tornei. $\dot{E}$ un cordone circolare intrecciato con quattro piccoli nodi alternati con altri quattro più grandi ed avente le estremità fioccate. Questo laccio fu detto anche nodo di Savoja, sull'origine del quale varie sono le opinioni degli eruditi. 


\section{CAPITOLO XII.}

IMPRESE E GRIDI DI GUERRA.

Le imprese o divise sono figure o motti, o le une o le altre insieme, che esprimono in un modo allegorico e breve qualche pensiero o sentenza.

La posizione delle imprese nelle armi è questa; se sono semplici motti si pongono in una fascia sotto lo scudo.

Se sono imprese di corpo e di anima, cioè composte di figure e parole, si pongono in cimiero.

Sulle imprese compose un eruditissimo ragionamento Monsignor Paolo Giovio (I); e ne trattò elegantemente anche Girolamo Ruscelli, in un discorso intorno alle invenzioni delle Imprese, delle Insegne, de' Motti e delle Livree (2); libri utilissimi a conoscersi da un italiano studioso dell'araldica e delle arti cavalleresche.

(I) Vedilo ristampato nella Biblioteca rara del Daelli. Milano, 1863

(2) Stampato in Venezia, 1556, appresso Giordano Filetti. 


\section{IMPRESE.}

Il Giovio ridusse a cinque le condizioni che dee avere una perfetta impresa. "Prima, giusta proporzione di anima e di corpo. Seconda, ch'ella non sia oscura, di sorte ch'abbia mestiero della Sibilla per interprete a volerla intendere: nè tanto chiara che ogni plebeo l'intenda. Terza, che sopratutto abbia bella vista, la qual si fa riuscire molto allegra, entrandovi stelle, soli, lune, fuoco, acqua, arbori verdeggianti, istrumenti meccanici, animali bizzarri, ed uccelli fantastici. Quarta, non ricerca alcuna forma umana. Quinta, richiede il motto, che è l'anima del corpo, e vuole essere comunemente d'una lingua diversa dall'idioma di colui, che fa l'impresa, perchè il sentimento sia alquanto più aperto. Vuole anco esser breve, ma non tanto, che si faccia dubbioso; di sorte che di due o tre parole quadra benissimo, eccetto se fosse in forma di verso, o integro o spezzato. » Non si stimano perfette quelle imprese consistenti o nella sola anima o nel solo corpo.

Cesare Borgia usò una impresa senza corpo, adottando il motto: aut Caesar aut nibil. La casa di Clermont: Si omnes ego non.

Riferiamo due imprese italiane criticate dal Giovio, la prima mancante di chiarezza e di dignità, la seconda di brevità.

Certo messere Agostino Porco da Pavia, innamorato di madonna Bianca Paltimera, per dimostrare di essere servo fedele di lei, portava una piccola candela di cera bianca nel frontale del suo berretto di scarlatto per significare, spezzando il nome della candela in tre sillabe, io sono can de la Bianca. 
Castruccio, Signor di Lucca, fatto senatore romano, comparve in pubblico in un manto cremisino con un motto di ricamo in petto, che diceva egli $\dot{e}$ come Dio vuole, e di dietro ne corrispondeva un altro, e sari quel che Dio vorrà.

Tra le strane si cita quella di un cavaliere spagnolo, il quale volendo dimostrare che egli era preso intieramente dall'amore della sua dama, senza sentirne nessun rimorso, adottò per impresa un diavolo col motto, mas perdido y menos arrepentido.

Si trovano delle imprese formate colle sole lettere alfabetiche.

Federico III trasse la sua dalle vocali A E I O U: esprimenti Aquilae est imperium orbis universi.

Casa Reale di Savoja ha il f.e. r. t. di cui si danno le più varie interpretazioni, la più conosciuta delle quali è: fortitudo ejus Rodbum tenuit.

Le italiane sono rare; ma se ne potrebbero fare delle bellissime, se i compositori delle medesime seguissero lo stile del Giordani nell'epigrafia italiana.

Eccone alcune: Un ferro sopra un'incudine: Mentre è caldo.

Sole velato da nubi: Tollerando sprezza.

La cometa: Empie gli occloi di luce, il cuor di gelo.

Una granata militare: Gara a cloi tocca. (Del duca Giovanni di Borbone).

Sulla granata svampante Lodovico Ariosto diè il motto latino al duca Alfonso di Ferrara, e fu, loco et tempore; convertito poi in lingua francese d lieu et temps. Stupende e più frequenti le latine.

Il tempio di una Fede, tutto rovinato: In me manet et ego in ea. 
Un giogo spezzato: Ramnusia rupit.

Una meta all'antica: It dolor ultra.

Storica e bellissima è quello del signor Fabrizio Colonna, il quale, scrive il Giovio, perseverando nelle parti francesi, invitato a seguire quelle italiche con gran premio, pose per impresa sulla sopravveste un vaso antico pieno di ducati d'oro, con questo motto: samnitico non capitur auro, volendo significare che egli come il Fabrizio romano non voleva essere corrotto dall'oro di re Pirro.

Anche il capitano Muzio Colonna, nepote di Fabrizio Colonna, descrisse felicemente il motto del romano Muzio Scevola, facendo per impresa una mano che abbruciava nel fuoco di un altare da sacrificio col motto: Fortia facere et pati romanum est.

Insegna il Ruscelli che i versi e i mezzi versi poetici si accordano leggiadramente alle imprese; e cita ad esempio di questi ultimi, il Fata viam invenient di Cesare, e il mezzo verso del Petrarca, impresa di Curzio Gonzaga, Chiusa fiamma è piu ardente.

E Luigi Gonzaga, prode capitano, venuto all'assedio di Roma col Borbone portò per impresa, il tempio di Efeso incendiato col motto, sive bonum sive malum fama est.

$\mathrm{Ci}$ sembrano elegantissime anche le imprese seguenti:

Una rosa col gambo spinoso: spinosa suavior.

Le bilancie: nec citra nec ultra.

Il re delle api: nec irasci quidem.

Stendardo militare: sta volteggiando e incuora.

Diaspro faccettato: unus sed tricolor.

L'Iride: splendide mendax.

Una sfinge: devine ou je te divore. 
Un fulmine che abbatte un tempio: per vetitum.

Il Ménéstrier divide le imprese in otto classi(I).

I. ${ }^{2}$ Imprese di cifre e rebus. Esempio: Un cavaliere italiano avendo perduto il premio in un torneo perchè il suo cavallo inciampò, comparve nella lizza il giorno dopo, con un formaggio o cacio secco sull' elmo per dire, oh caso duro!

$2 .^{2}$ Imprese dal nome della famiglia: Belly $-D \imath-$ bius eventus BELLI.

Morlaix. - S'ils te mordent, mors-les.

Savoja Nemours. - Suivant sa-voye.

Crescenzio. - Aspice crescam.

Alba di Toledo. - Al parecer de l'alba s' ascondan las estrellas.

3. Imprese allusive all'arme.

Grifeo. - Nell'arme un grifo, e il motto: noli me tangere.

Paleologo. - Nell'arme una croce, e il motto: In boc signo vincet.

Rochechouart. - Arme fasciata, ondata e il motto: Avant que la mer füt au monde, Rochechouart portait les ondes.

4. ${ }^{a}$ Imprese enigmatiche.

Croy (Giovanni di). - Souvienne vous.

5. Imprese di proverbi, sentenze, e parole.

Grillo. - Nitimur in vetitum.

Gran Bretagna. - Dieu et mon droit.

Scozia. - Pro lege et pro rege.

Belgio. - L'union fait la force.

(I) Ménéstrier, Origine des ornements des armoiries. 
San Marino. - Libertas.

Coucy. - Je ne suis roy ni prince aussi, je suis le sire de Coucy.

Ricasoli. - Cum bonis bonus, cum perversis perversus.

Merode. - Plus d'bonneur, que d'bonneurs.

Byron. - Crede Byron.

Créqui. - Créqui baut baron, Créqui baut renom. Tapparelli d'Azeglio. - O mater Dei memento mei. Gravina. - Spero.

Chateaubriand. - Mon sang teint les bannières de France. Rohan. - Roy ne peux, duc ne veux, Roban suis.

6. ${ }^{a}$ Le imprese storiche sono quelle che traggono origine da qualche fatto istorico, o da qualche motto celebre profferito da qualcuno della famiglia che 1o scelse a divisa, o ad esso rivolto.

Colombo (Cristoforo). - Por Castilla y por Leon muevo mundo ballo Colon.

Beaumanoir. - Bois ton sang, Beaumanoir, ta soif passera.

7. Imprese composte di sole figure, cioè senza il motto: queste figure si pongono per lo più in cimiero, quindi sono dette anche pezze da cimiero.

York. - La rosa bianca.

Lancastro. - La rosa rossa.

Tudor. - Una rosa partita bianca e rossa.

La 8. ${ }^{a}$ infine comprende le vere e proprie imprese o divise, di cui abbiamo già accennato le principali regole.

GRIDO DI GUERRA E D'ARME.

Il grido di guerra, scrive il Ginanni, è un motto ristretto in una, due o tre parole, posto al disopra del- 
l'arme in un listello svolazzante; è detto grido, perchè i capitani ed i duci se ne servivano anticamente per unire le loro schiere e condurle all'assalto.

Il grido di guerra è proprio dei sovrani, e i cadetti non hanno il diritto di portarlo.

Il grido d'arme rimonta alla più alta antichità, anche innanzi alla Cavalleria, come ne fanno fede i clamori guerreschi dei Galli e dei Germani detti Bardit perchè composti dai bardi.

Dice il Ménéstrier che il grido di guerra segue la bandiera: allora, nel periodo cavalleresco, i soli cavalieri banderesi, gentiluomini di nome e d'arme o di grido, avevano il diritto di spiegare la bandiera; quindi tanti gridi quante bandiere.

Varie sono le classificazioni che hanno dato gli araldisti dei gridi di guerra; il più autorevole, il Ménéstrier, li divide in otto categorie, distinte ciascuna per lo scopo del grido cioè: di decisione o di risoluzione, d'invocazione, di sfida, di combaitimento, d'esortazione, di gioja, d'avvenimento e di raccolta.

Il Crollalanza crede che vi si potrebbero aggiungere $i$ gridi di pretensione, di orgogtio, di amore, e quello allusivo all'arma.

Noi daremo pochi esempi di ciascuna di queste categorie:

I. I primi crociati. - Dieu le volt!

2. Montmorency. - Dieu aide au prentier baron critien!

Du Guesclin. - Nostre-Dame Guesclin!

Austria. - Nostra Signora alla riscossa!

Francia. - Montjoie et Saint Denis!

Inghilterra. - Montjoie Saint-Georges! 
Spagna. - Santiago.

Stati della Chiesa. - San Pietro!

Savoja. - Saint-Manrice!

3. Foix (Febo di). - Touches y si tu l'oses! Coucy-Chateauvieux. - Place à ma bannière!

4. ${ }^{\circ}$ Austria. - A destra e a sinistra!

Bar. - Au feu au feu!

5. Sciampagna (Conti di). - Passavant li meillor! - Passavant là Tibaud!

Genlis. - Au guet! au guet!

6. Rosièrs. - Grand joie!

Rabiers. - Victoria!

Moussaye. - Honneur à Moussaye.

7. Savoja. - Bonnes nouvelles!

Coucy. - A la merveille!

8. Savoja. - Savoye, Savoye!

Fiandra (i Conti). - Flandres au lion!

Grido di pretensione:

Imperatore Ottone. - Roma!

Grido di orgoglio:

Blacas. - Vaillance!

Des Monts. - Fortis ut mons!

Napier. - Sans tache!

Grido di amore:

Aleman. - Place, place à madame!

Grido allusivo all' arme.

Casa di Waudripont nell'Hainaut - Cul à cul Waudripont! perchè porta due leoni addossati nello scudo. 


\section{CAPITOLO XIII.}

BRISURE.

Il vocabolo brisura proviene dal francese verbo briser, che vuol dire rompere, spezzare. Nel linguaggio blasonico la brisura o spezzatura indica l'alterazione di un'arma gentilizia per distinguere i diversi rami di una famiglia, o le linee bastarde.

Si debbono considerare introdotte le brisure nei tempi in cui le armi furono rese invariabili ed ereditarie, cioè nell'epoca delle Crociate (I096-I270).

Pare che la necessità e non il capriccio introducesse l'uso delle brisure.

Quando i combattimenti si facevano ad armature chiuse, nota il Crollalanza nella sua Enciclopedia-Araldico-Cavalleresca, era estremo il bisogno fra i membri di una stessa famiglia il distinguersi con segnali particolari, e poichè tutti avevano l'arma stessa colla stessa livrea della loro casa, dovevano di conseguenza adottare contrassegni speciali pei quali si riconoscessero.

Crebbe il bisogno delle brisure colla istituzione dei 
majorascati e degli appannaggi, avendo il solo capo della famiglia il diritto di portare l'arme della casata pura, senza nessuna alterazione; mentre i suoi figli, compreso il primogenito, vivente il padre, avevano l'obbligo di distinguere le loro armi colle brisure.

I figli naturali dovevano portare sul loro scudo, una sbarra, una traversa, un filetto di bastardume, o altro contrassegno della loro illegittimità.

Nè questa illegittimità impressa nelle armi gentilizie dispiaceva allora ai nobili, come si vede dagli scudi dei bastardi di Orléans, di Longueville, di Lussemburgo e di altre celebri case.

Ai nostri tempi, aboliti i majorascati, le brisure si sono rese quasi inutili, e restano solamente alle famiglie sovrane. La Consulta Araldica Italiana ha però, non sappiamo con quanta opportunita, rinnovato l'uso della spezzatura.

Una regola da seguirsi nelle brisure è quella insegnata dal Bouillet il quale dice, che le migliori brisure sono quelle che alterano il meno possibile le pezze primitive.

Varie sono le maniere di brisare lo scudo:

I. Cangiando le figure e conservando gli smalti;

2. Cangiamento delle figure;

3. Permutazione degli smalti;

4. Alterazione del numero delle figure uguali;

5. Alterazione delle posizioni delle figure;

6. Alterazione delle forme delle figure;

7. Omissione di qualche figura;

8. ${ }^{\circ}$ Addizione di altre figure;

9. Partizioni e inquartature. 
Colla prima, la più antica, si conserva la livrea della famiglia e l'origine della casata.

La seconda produce confusione a causa della somiglianza delle figure araldiche in molte famiglie.

La terza, che si trova in uso più frequente in Italia, c̀ meno imperfetta delle prime due.

La quarta si presta meglio allo studio degli araldisti, alterando pochissimo l'arma primitiva e conservandone gli smalti.

La quinta si produce cambiando la situazione e la giacitura di una figura.

La sesta ha il vantaggio di potere facilmente alterare la pezza semplice in numerosissimi modi.

L'ottava è quella più in uso, perchè, introducendo nell'arme piena, alcune date figure riconosciute come pezzi di brisura, non altera in nulla l'arme primitiva e ne facilita il riconoscimento.

I principali pezzi di brisura riconosciuti sono: il lambello, la bordura, il bastone scorciato, la cotissa, il filetto in sbarra, le stelle, i bisanti, gli anelletti, i merlotti, lo scudetto, la conchiglia, il cantone, ecc.

La nona maniera nacque dai titoli feudali appartenenti ai rami cadetti delle famiglie. Essi inquartavano o partivano l'arma di appannaggio con quella pura di famiglia.

Vi sono ancora due altri modi di brisura, cioè dei cimieri e dei supporti.

In Germania la brisura si fa consistere nella differenza del timbro.

In Inghilterra si brisano $i$ supporti.

Nella Spagna la brisura principale è la bordura.

In Francia in cui le brisure si conservano per molto 
tempo nelle più illustri famiglie, secondo il Cassaneo, venivano distribuite cosi: al secondogenito, il lambello; al terzogenito, la bordura semplice; al quartogenito, la bordura dentata, merlettata, bisantata o scanalata: agli altri cadetti il bastone più o meno largo, oltre la bordura. 


\section{CAPITOLO XIV.}

\section{LEGGI ARALDICHE.}

Le leggi intorno alla composizione delle armi sono comuni a tutti i paesi in cui è conosciuto il blasone. Gli araldisti si sono preso cura di riferircele, e spesso anche di inventarne. Il Crollalanza, che ne dimostra per quasi tutte l'inanità e la falsità, ha raccolto nella sua Enciclopedia Araldico-Cavalleresca le seguenti:

I. Non si deve mai porre metallo sopra metallo, o colore sopra colore. I capi cuciti, le brisure, e le appendici delle figure fanno però eccezione a questa legge, che è una delle poche realmente osservate e giustificate.

2. Le pelliccie, la porpora e 1 e convenevoli partizioni possono figurare come metallo e come colore.

3. Il seminato di Francia ha lo stesso privilegio.

4. Gli animali si devono porre nella posizione più nobile e più conveniente alla loro natura.

5. Il piede anteriore destro degli animali passanti o rampanti deve sempre andare avanti al sinistro.

6. Gli animali si devono mettere, salvo qualche eccezione, rivolti a destra. 
7. Le armi più semplici e meno caricate sono le più belle, in virtù di un antico proverbio araldico che chi ba piu ba meno.

8. Le migliori armi sono quelle composte di figure araldiche o degli animali più nobili.

9. Il metallo deve stare nel luogo più nobile dello scudo, ossia nel primo quarto dell'inquartato, nel primo pezzo del fasciato, del palato, ecc., a destra nel partito, ecc. Questa legge è falsa, e condannata, oltrechè da un gran numero di araldisti, anche dall'uso generale.

Io. Le arme dovrebbero essere tutte di figura di metallo su campo di colore, perchè gli scudi antichi erano di legno o di cuoio. Anche questa legge è falsa come la precedente.

II. Le figure non devono toccar la punta dello scudo, nè sostenersi sopra zolle, terrazze e simili, ma campeggiare isolate. Benchè questa regola sia poco osservata, è una delle meglio ispirate allo stile araldico puro.

12. Quando vi sono molte figure in uno scudo, se ne pongono piu in capo che in punta; ragione per cui la disposizione araldica è 2 e I; 3, 2 e I; 5, 4, 3, 2 e I; ecc. Cosi pure tutte le figure devono avere maggiore sviluppo verso il capo che verso la punta. Ciò si spiega pel fatto che gli antichi scudi erano triangolari, ed aveano maggior larghezza in alto che in basso.

13. Non si introducano nell'armi figure umane intere. I Tedeschi sono quelli che meno osservano questa legge.

14. Molti araldisti escludono dall'arme le galline, i porci, le pecore, le lepri, le oche, gli asini, i conigli ed altri animali che dicono ignobili. Ma l'arme di molte 
illustri ed antiche famiglie contraddicono a questa pretesa.

I5. Le arme non devono avere più di tre figure principali di specie diversa. Gli Spagnuoli sono quelli che meno osservano questa regola.

r6. Le concessioni siano poste nel luogo stabilito, e le figure di padronanza nel posto più nobile.

17. L'arma non abbia più di tre smalti, nè meno di due.

I8. Nel blasonare si comincia dal campo, poi si nominano le figure, il loro smalto, la loro situazione, il loro numero, i loro attributi, ecc.

La descrizione che comincia colle figure e finisce col campo è antiaraldica.

I9. Basta blasonare la composizione dello scudo per dar l'arma d'una famiglia; il resto non è che accessorio, e può variare a seconda delle circostanze.

Diconsi alterazioni quei cambiamenti portati dai varî stili alle figure araldiche primitive. Così i gigli di Francia, le rose, i vepri, i raggi di carbonchio, i rocchi di scacchiere, le mandorle pelate, e tante altre, sono figure alterate.

Diconsi combinazioni le pezze e figure araldiche risultanti dall'unione di altre pezze; sono combinazioni il capo-palo, la fascia-cantone, il capo-banda, il capocapriolo, ecc.

Diconsi contrazioni gli scorciamenti di una pezza: cosi l'amaide è una contrazione della fascia, la crocetta una contrazione della croce, ecc.

Diconsi modificazioni le varianti di pezze o figure alterate nei loro contorni da attributi che ne alterano i contorni. Sono modificazioni tutte le croci che differi- 
scono dalla croce piana, le pezze ondate, increspate, merlate, spinate, potenziate, ecc.

Diconsi recessioni le pezze onorevoli ritirate, cioè che hanno una estremità staccata dal bordo dello scudo.

Diconsi riduzioni le pezze rappresentate in proporzioni più strette delle normali. Il colmo, la verghetta, la burella, la trangla, la riga, la cotissa, la divisa, il bastone, il filetto, la traversa, lo scaglionetto, l'estrez, ecc. sono riduzioni.

Le positure o situazioni delle figure si distinguono in: I. fisse, che non si esprimono nel blasonare, perchè occupano il posto che è loro assegnato dall'uso costante; 2. irregolari, come una campagna obliqua, un capo abbassato, un albero in fascia, una pergola in sbarra, ecc.; $3 .^{\circ}$ di somiglianza, quelle delle figure disposte nel senso e a similitudine della fascia, della banda, della croce, della cinta, ecc.; $4 .^{\circ}$ reciproche, quelle delle figure considerate rispetto ad altre, come figure affrontate, addossate, attraversanti, accostate, sormontate, addestrate, ecc.; 5. alternate o di contrapposizione, quando gli smalti che distinguono le figure si contrappongono alternativamente, ciò che si esprime coi termini dell'uno all'altro e dell'uno nell'altro (I).

(I) Ved Gueifi, Vocabolario Araldico, a queste due voci. 


\section{CAPITOLO XV.}

Regole generali per blasonare.

Blasonare è descrivere le armi secondo i principî e le regole dell' arte araldica, e servendosi del lingurggio tecnico del blasone.

Queste regole sono minutamente e chiaramente raccolte ed esposte dal Crollalanza nella sua Enciclopedia Araldico-Cavalleresca alla voce Blasonare. In un trattatello elementare come questa Grammatica noi dobbiamo limitarci a darne un'idea sommaria.

S'incomincia da indicare il campo dello scudo, quindi si passa alle figure; per esempio:

D'argento, alla banda d'azzurro, caricata di tre stelle a sei raggi d'oro, e accompagnata in capo d' una testa di leone al naturale.

Se lo scudo è composto, si blasona prima la partizione che lo divide, e gli smalti e le figure di ciascun quarto.

Per es.: Partito: nel I. d'oro, all'aquila bicipite spiegata di nero, inembrata, imbeccata e coronata del campo, uscente dalla partizione; nel $2 .^{\circ}$ d'oro, al cane rampante di nero. 
Partito, indica la partizione; al primo, il quarto destro: al secondo, il quarto sinistro dello scudo.

Se lo scudo è spaccato, si comincia dalla parte superiore.

Se è tagliato o trinciato si principia a blasonare dal triangolo superiore.

Se è inquartato o controinquartato, si blasona cominciando dalla prima parte di destra e si termina all'ultima parte di sinistra.

Per es.: inquartato al $\mathrm{I} .^{\circ}$ e al $4 .^{\circ}$ d'argento, alla $f a-$ scia di rosso; al 2. d' azzurro, al palo d'oro; al $3 .^{\circ} d i$ rosso, alla croce di argento.

Quando uno scudetto si trova in mezzo all'inquartatura, cioè sul tutto, si blasona dopo di avere blasonato tutti i quarti dello scudo principale.

Allorchè lo scudo è diviso in otto quarti si blasona cominciando sempre dal primo quarto di destra.

Per es.: tagliato d'uno, partito di tre. Lo scudo può essere partito di $2,3,4,5$ linee secondo il numero dei quarti.

Per es.: tagliato d'uno, partito di tre, al.$^{\circ}$ e $6 .^{\circ}$ di azzurro, al $2 .^{\circ}$ e al $50^{\circ}$ d' argento, al $30^{\circ}$ e all' $8 .^{\circ}$ di rosso, al $4 .^{\circ}$ e al $7 .^{\circ}$ d'oro.

$\mathrm{Fa}$ d'uopo indicare con precisione tutte le figure che ricuoprono lo scudo, sia che carichino o accompagnino le principali, in particolar modo, se la loro posizione esce fuori delle regole.

Per es.: Tre bisanti d'argento in campo azzurro, due al capo e uno in punta, si blasonano due e una.

Per es.: d'azzurro, a tre bisanti d'argento posti due e uno.

Se sono posti l'uno sotto l'altro, diconsi situati in palo. 
Posti orizzontalmente diconsi in fasia, in banda, in sbarra, ecc., secondo che sono disposti a somiglianza della banda, della sbarra, del palo, ecc.

Per es.: d' argento a tre bisanti d'argento situati in palo.

Di nero a tre bisanti d'oro situati in sbarra.

Blasonato lo scudo si passa all'elmo e alla corona o a qualunque segno di dignità che figuri sullo scudo.

Allora si dice: Scudo, timbrato dell'elmo di principe, duca, nobile, ecc., o delle relative corone; e si descrivono anche i lambrecchini e il cimiero, i supporti, la divisa e il grido di guerra. 


\section{APPENDICE}

DELLE LIVRÉE.

La parola livréa non è italiana come la parola divisa, la quale, secondo il dottissimo Muratori, derivò dalle sopravvesti di due differenti colori indossate dai nobili giovani nei giuochi e negli spettacoli dei tempi di mezzo (I).

Il vocabolo livréa provenne da quello francese livrie, equivalente al latino liberalia, liberare, perchè anticamente era un contrassegno con cui un signore feudatario liberava i suoi sottoposti da ogni gravezza, concedendo loro un abito coi colori del suo stemma. I re franchi della seconda razza chiamarono livrée anche certe vesti uniformi e di gran valore da essi distribuite ai grandi uffiziali delle loro corti.

Coll'andar del tempo invece di coteste vesti abbero dalla casa del re, secondo il loro grado, una somma

(1) Antiquit. ital. dissert, 29. - V. l'eruditissimo e completo trattato dellillustre Commendatore Cario Padigi.ione, Delle livrée e del modo di comporle, e descri־ione di quelle di faniglie nobili italiane. Napoli, 1889 , in-So grande. 
di denaro detta livréa; e quest'uso durò sino alla rivoluzione.

Livrèa fu detta altresi una sciarpa portata dai cavalieri e dai feudatari a traverso le loro armature, e questa serviva a farli riconoscere di lontano.

Caduto l'uso delle cotte di armi, i baltei e le sciarpe, le livrée passarono dai signori ai servitori. In Francia, sotto il primo Impero e la Restaurazione, le livrée furono di concessione sovrana. Luigi Filippo riservando il colore rosso per la real casa, lasciò libertà a tutti di metter su livrée a piacere.

Le livree si divisero dagli araldisti in due specie, livrée di onore e di servitù.

Le prime erano gli abiti portati dai sovrani e dai gran signori le quali, con differenza di stoffa e di ornati imponevano pure al loro seguito; le assise dei militari, le vesti di gala dei magistrati, dei professori, degli avvocati, le divise degli ordini cavallereschi; gli abiti a brevetto (inventati da Luigi XIV), il vestiario da caccia a invito, ecc.

Le livrée di servitù erano di diverse qualità: livrée di un sol colore, listate, gallonate, frastagliate, ecc., ed erano portate dai famigliari e dai servitori delle nobili case.

In Italia come in tante altre cose si seguitò generalmente il costume francese; dico in generale, perocchè apparisca da un passo del Borghini come a Firenze alcune famiglie usassero i colori delle livrée diversi da qué della loro arma.

Il Memoriale della nostra Consulta Araldica non fa menzione delle livrée.

Poche sono le regole per comporre le livrée. 
Gli smalti, i colori e i pellicci delle livrće si cangian nei colori delle livrée nel modo seguente:

L'oro - in giallo o arancio.

L'argento - in bianco o cenerino.

L'armellino - in felpa nera.

Il vajo - in felpa quadrigliata, azzurra e bianca.

Il palato, il fasciato, il bandato e lo sbarrato si traducono in liste verticali, orizzontali e diagonali dei due colori alternati. Il losangato elo scaccato richiedono uguale ripartizione di colori.

Il colore o i colori principali dello scudo devono essere anche quelli della livréa; della quale le parti più importanti, araldicamente parlando, si ravvisano nel giustacuore o panciotto, dai francesi detto gilel, e nei calzoni, corti o lunghi che siano.

Le partizioni si fanno adottando il primo colore per campo, e gallonando o bordando col secondo colore; quindi è di regola, che il primo smalto di una partizione sia sempre quello del giustacuore.

Per l'inquartato sta la medesima regola.

Il giustacuore sarà sempre il fondo del primo colore, e la bordatura del secondo.

Nel panciotto dunque dere campeggiare il colore principale dello scudo e nei calzoni il colore della figura principale.

Se lo scudo fosse pieno, di un solo colore, panciotto e calzoni si faranno dello stesso colore.

La sopraveste o soprabito si usa farsi dello stesso colore dei calzoni: e se il colore ne fosse troppo avventato si sceglie, insegna Goffredo di Crollalanza (I),

(1) Enciclop. Araldico-Cavalleresca. 
una stoffa di una tinta approssimativa, come per il rosso, il color marrone, per il verde, il verde ruggine, ecc.

In quanto ai bottoni si adopreranno d'oro o dorati, se il fondo dello scudo è di questo metallo, e di argento o inargentati se è d'argento.

I bottoni comunemente sono marcati dell' arme padronale o delle iniziali coronate o semplici secondo che piace.

Si costuma ancora, e in Italia specialmente dai prelati, di bordare la livréa con galloni nei quali sono intessute o ricamate le armi della casa.

Facili sono le regole per la bordura o galloni.

Uno scudo senza figure richiede i galloni di 5 centimetri di larghezza.

Uno scudo con una pezza onorevole, come la croce, la fascia, la banda, ecc., si mette di 4 centimetri.

Per tutti gli altri scudi si debbono adoperare i galloni di soli 3 centimetri, e si ritengono comporsi di figure ordinarie come la losanga, il bisante, ecc., o di figure di corpi naturali, o di figure artificiali o di fantasia.

Con queste poche regole, scrive il Gourdon di Genouillac(I), è facile conoscere il carattere principale dell'arme di una casa.

Dai bottoni si arguisce se il fondo dello scudo è di metallo.

Dal gallone si conosce se lo scudo contiene una pezza onorevole, o una ordinaria, o una figura.

Dal colore dei calzoni se ne impara lo smalto.

(1) Traitê sur la composition des livrées. 
La scienza araldica, nota egli, composta di emblemi e di simboli, spiega i suoi enimmi per mezzo degli uni e degli altri, ed è come la frammassoneria della nobiltà. Diamo ora alcuni esempi pratici per comporre e blasonare le livrée.

Se la casa spiega d'oro..., sceglierà per la sua livréa il panciotto giallo e il gallone di s centimetri; se di vajo il panciotto sarà di felpa quadrigliata di azzurro e bianco, e il gallone di 3 centimetri.

Se lo scudo è spaccato d'oro e d'azzurro, il panciotto sarà di stoffa gialla bordato di azzurro e il gallone di 3 centimetri.

Se invece è partito di rosso e d'oro, sarà rosso bordato di giallo, e il gallone di 3 centimetri.

Se poi fosse uno scudo inquartato, per es., nel $\mathrm{I} .^{\circ} \mathrm{e}$ $4 .^{\circ} d^{\prime}$ azzurro e nel $2 .^{\circ}$ e $30^{\circ}$ d'argento, il panciotto sarà turchino bordato di bianco, e galloni di 5 centimetri. Sarebbe inutile moltiplicare gli esempi.

FINE. 



\section{0}

\section{MANUALI HOEPLI}

Pubblicati a tatto 0ttobre 1903.

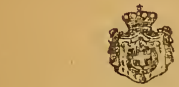

Ministero dell' Istruzione

Gabinetto

del Sottosegretario di Stato

Roma, 3 nov. 1900.

III.mo Signore

Comm. Uirico Hoepli

Editore

Milano.

La collezione dei Manuali Hoepli, ricca ormai di quasi 700 volumi, forma la più vasta enciclopedia di scienze, lettere ed arti finora apparsa in Italia. Meritano lode certamente e gli autori, che in forma lu. cida e breve hanno preparato cosi valido ausilio alla gioveniti studiosa, e l'editore che ha saputo scegliere, tra le varie discipline, quelle che meglio valgono a formare un complesso di cognizioni indispensabili alla cultura moderna.

\section{firmato:}

\section{Enrico Panzacchir.}

Sotto Segretario di Stato Ministero della Pubbl. Istruzione.

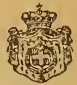

II Ministro

per l'A gricoltura, l'Industria e il Commercio

Poma, 25 ott. 1900.

Ill. sig. Comm. U. Hoepli, Milano.

La larga accoglienza fatta alla collezione dei manuali. $e$ diti dalla Sua benemerita Casa, deve certo formare la migliore e più ambita ricompensa per la S. V. Ill.ma, che con intelligente cura ne dirige la pub. blicazione.

Questo Ministero ha avuto piu volie occasione di fermare la sua attenzione sui lavori che più direttamente riguardano l' agricoltura, la zootecnia e le industrie ad esse atitinenti, tro. vandoli rispondenti allo scopo, che la S. V. Ill.ma si propone di conseguire.

Mi torna quindi gradito di esprimerne a Lei il mio sincero compiacimento, mentre Le auguro che sempre maggior favore abbia ad incontrare code. sta Sua utile raccolta

firmato: Carcano. SCin. dell'Agr., Ind. e Comm. 


\section{A.VVERTEIJZA}

Tutti i MANUALI HOEPLI sono elegantemente legati in tela e si spediscono franco di porto nel Regno. - Chi desidera ricevere i volumi raccomandati, onde evitare lo smarrimento, è pregato di aggiungere la sopratassa di raccomandazione.

DE I libri, non raceomandati, viaggiano a rischio pericolo del committente 


\section{Manuali Hoepli Divisione sistematica.}

Nella presente divisione sono radunate in pochi gruppi e disposte in ordine alfabetico tutte le voci piu salienti delie materie trattate nei Manuali Hoepli e prego gli Studiosi di consultarla sempre nelle loro ricerche.

\section{Agraria.}

Abitazioni d. animali Distillazione vinacce |Molini

Agricoltore(il lib.dell') Economia fabb. rurali

Agricoltore (pront. d.) Enologia

ed.ingegnere rurale Agronomia

Id. e agricoltura Agrumi

Alimentaz. bestiame

Analisi vino

Animali da cortile

Id. parassiti

Apicoltura

Assicur. aziende rurali

Bachi da seta

Bestiame e agricolt.

Campicello Scolastico

Cane

Cantiniere

Caseificio

Catasto

Cavallo

Chimica agraria

Cognac

Colombi domestici

Gompntisteria agraria

Concimi

Coniglicoltura

Id. domestica

Estimo rurale

Id. dei terreni

Floricoltura

Fosfati, perfosfati

Frumento e mais

Frutta minori

Frutticoltura

Funghi mangerecci

Gelsicoltura

Humus

Igiene rurale

Id. veterinaria

Immunità a. malattie

Insetti nocivi

Id. utili

Latte, burro o cacio

Legislaz. rurale

Macchine agricole

Mais

Majale

Malattie crittogam.

Malattie dei vini

Mezzeria
Mosti e vini (densità d.)

Olivo e Olio

Olii vegetali, ecc.

Orticoltura

Panificazione

Piante e fiori

Piante industriali

Piante tessili

Pollicoltura

Pomologia

Prato

Prodotti agr.d.Tropico

Razze bovine, equine, ecc.

Selvicoltura

Sofisticazione vino e aceto

Tabacco

Tartufi e funghi

Triangolaz. Top.e Cat.

Uve da Tavola

Vini bianchi

Vino

Viticoltura

Zoonosi

Zootecnia

\section{Prodotti alimentari.}

Adulteraz. alimenti

Agrumi

Alimentazione

Animali da cortile

Apicoltura

Aromatici e nervini

Caseificio

Cantiniere

Cognac

Colombi domestici

Coniglicoltura

Conservazione sostan$z e$ alimentari
Olivo e olio

Olii vegetali

Orticoltura

Ostricoltura

Panificazione

Piscicoltura

Pollicoltura

Tartufi e funghi

Uve da tavola

Vini bianchi

Vino 


\section{Industrie diverse.}

Abiti per signora Acetilene Acido solforico Alcool industriale Apicoltura

Arti grafiche

Asfalto

Bachi da seta

Biancheria

Carta (Industria d.)

Cognac

Colori e vernici

Commerc. (Man, del) Commercio (Storia d.) Concia pelli

Distillazione del legno

Id. delle vinacce

Elettricità e appl. vedi al gruppo Elettricità

Fabbro ferraio

Falegname ebanista,

Filatura e tessitura

Fiori artificiali

Fonditore di metalli

Fotografia:

Carte fotografiche
Fotografia :

Dizionario fotogr.

Fotocromatografia

Fotog. industriale

Fotog. ortocromat.

Fotog. p. dilettanti

Fotogrammetria

Fotosmaltografia

Processi fotomecc.

Proiezioni fotog.

Ricettario fotog.

Spettrofotometria

Gaz illuminante

Gioielleria, oreficeria

Imitazioni e succedanei

Incandescenza a gaz

Industria frigorifera

Litografia

Macchine per cucire

Marmista

Meccanica

Meccanico

Metalli preziosi

Modellatore meccan.

Naturalista preparat. Operaio
Orologeria

Ostricoltura

Panificazione

Piante industriall

Id. tessiii

Piccole industrie

Pietre preziose

Pirotecnia

Piscicoltura

Pomologia artificiale

Ricettario domestico

Id. industriale

Saggiatore

Saponi (Industria dei)

Seta (Industria d.)

Specchi (Fabbric.)

Stearica (Industria)

Tessuti di lana e cot.

Tipografia

Tintore

Tintura della seta

Tornitore meccanico

Trine a fuselli

Vernici, lacche, inch.

Vetro

Zacchero

\section{Fisica $\theta$ Chimica.}

Acetilene

Acido solforico

Adulterazione alim.

Alcool

Analisi chimica qual. Analisi vino

Id. volumetrica

Calore

Chimica

Id. agraria

Id. analitica

Id. appl. a.igiene

Id. clinica

Id. legale

Chimico industriale

Climatologia

Cognac

Concimi
Conservaz. sost. alim.|Gravitazione

Dinamica

Disinfezione

Distillazione del legno

Id. delle vinacce

Elettrochimica

Energia fisica

Esplodenti

Farmacista

Farmacoterapia

Fisica

Fisica criatallografica

Fotografia (v. al gruppo Inżustrie)

Fulmini e parafulmini

Galvanoplastica

Galvanizzazione

Gaivanostegia
Igroscopi, igrom.

Latte, burro, cacio

Liquorista

Luce e colori

Id. e suono

Meteorologia

Microscopio

Olii veget. miner.

Ottica

Profumiere

Sieroterapia

Spettroscopio

Termodinamica

Tintore

Tintura di seta 


\section{Storia Naturale.}

Acque miner. e term. Fisica cristallografica Anatom. e fisiol. comp. Fisiologia

Anatomia microscop.

Anatomia vegetale

Animali parass. uomo

Antropologia

Batteriologia

Biologia animale

Botanica

Cane

Cavallo

Coleotteri

Colombi domestici

Coniglicoltura

Cristallografia

Ditteri

Fmbriol e morfol. gen.

Fiori artificiali

Floricoltura

Id. vegetale

Frutticoltura

Frutta minori

Funghi mangerecci

Geologia

Imenotteri ecc.

Insetti nocivi

Id. utili

Ittiologia

Lepidottexi

Majale

Malattie crittog.

Metalli preziosi

Mineralogia gener. Id. descritt.

Naturalista preparat.

Naturalista viaggiat.
Orticoltura

Ostricoltura e mitil.

Paleoetnologia

Paleontologia

Piante e fiori

Pietre preziose

Piscicoltura

Pollicoltura

Ponologia

Protistologia

Selvicoltura

Sismologia

Tabacco

Tartufi e funghi

Tecnica protistol.

Uccelli canori

Vulcanismo

Zoologia

\section{Medicina, Chirurgia, Igione.}

Acque miner. e term.

Analisi chimica qual. Anatomia e fis. comp. Anatomia microscop. Anatomia topograf. Animali parass. nomo Antropometria

Aromatici

Assistenza infermi Id.

Batteriologia pazzi

Biologia animale

Chimica appl. a. igiene Chimica clinica

Chimica legale (toss.)

Chirurg. operativa

Climatologia

Disinfez. (Pratica d.)

Embriologia

Epilessia

Farmacista

Farmacoterapia

H'isiologia

Fototerapia

Cavi telegrafici

Correnti elettr. altera.

Distillazione del legno

Elettricità

Elettrotecnica

Elettrochimica
Idroterapia

Igiene della bocca

Id. del lavoro

Id. vita pubblica

Id. della pelle

Id. privata

Id. rurale

Id. scolastica

Id. veterinaria

Id. della vista

Immunità malattie

Impiego ipodermico

Infortuni d. montagna

Legislazione sanitaria

Luce e salute

Malatt. dei paesi caldi

Malattie del sangue

Massaggio

Materia medica

Medicatura antisett.

Medico pratico

Microbiologia

Microscopio

Morte vera e app.

\section{EHlettricità.}

Fulmini e paratulmini Metallocromia

Galvanizzazione

Galvanoplastica

Galvanostegia

$\begin{array}{ll}\text { Galvanostegia } & \text { Telegrafia } \\ \text { Illuminazione elettric } & \text { Telegrafia senza fli }\end{array}$

Magnetis. e elettricità. Unità assolute
Nutrizione bamb.

Organoterapia

Ortofrenia

Ostetricia

Pellagra

Protistologia

Psichiatria

Psicologia fisiol.

Psicoterapia

Rachitide

Radioterapia

Röntgen (Raggi)

Semejotica

Sieroterapia

Soccorsi d'urgenza

Spettrofotometria

Terapia infanzia

Tisici e sanatori

Ufficiale sanitario

Veleni

Zoonosi
Röntgen (Raggi di)

Telefono

Telegrafia 


\section{Tecnologia, Ingegneria, Costruzioni, ecc.}

Abitazioni anim. dom.|Fabbro ferraio

Architettura

Aritmetica e Geom. op. Asfalto

Atlante di macchine

Automobilista

Calcestruzzo

Calci e cementi

Calderaio

Casa dell'avvenire

Case operaie

Ciclista

Coltivazione miniere

Conti e calcoli fatti

Cubatura legnami

Curve circolari

Decoraz. e indust. art.

Dinamica

Disegnatore meccan.

Disegno assonometr.

Id. geometrico

Id. industriale

Id. di projez. ort.

Id. (Gramm. del)

Dizionario tecnico

Fabbricati civili
Falegname-ebanista

Fognatura cittadina

Id. domestica

Fonditore in metalli

Fotogrammetria

Gaz illuminante

Gnomonica

Idraulica

Imitazioni e succed.

Incandescenza a gaz

Industrie (Piccole)

Infortuni sul lavoro

(Mezzi p. prevenirli)

Ingegnere civile

Ingegneria legale

Lavori marittimi

Lavori in terra

Leggi lavori pubblici

Leghe metalliche

Macchine a vapore

Id. agricole

Id. per cucire

Macchinista e fuochist.

Marmista.

Meccanica
Meccanico

Meccanismi (500)

Miniere

Modellatore meccanic.

Molini

Momenti resistenti

Montatore d.macchine

Operaio

Orologeria

Peso metalli

Prontuario d. agricoltore e d. ingegnere rurale

Prospettiva

Regolo ealcolatore

Resistenza d.materiali Scaldamento e ventil, Siderurgia

Stereometria

Strumenti metrici

Tavole d'alligazione

Tempera e cementaz.

Termodinamica

Tornitore

Zolfo

\section{Matematiche.}

Algebra elementare

Id. compl. I anal.

Id. Id. II equaz.

Id. (Esercizi di)

Aritmetica pratica

Id. razionale

Id. (Eserc. di)

Id. e geom. d. op. Astronomia

Id. nautica

Id. n. antico test.

Calcolo infin. I calc.diff

Id. II integrale

Id. III d. variaz.

Id. (Esercizi di)

Celerimensura

Compensazione errori Computisteria

Conti e calcoli fatti

Cubatura legnami

Curve circolari

Determinanti

Disegno assonometr.
Disegno geometrico

Id. industriale

Id. di projezioni

Id. topografico

Economia matemativa Matematica (compl.di)

Eserciz.d. geom. elem. Matematiche superiori

Id. di Trigonom.

Euclide (L') emendato

Formulario di matem.

Fotogrammetria

Funzioni analitiche

Id. ellittiche

Geometr.anal.d. piano

Id. Id. d. spazio

Id. descrittiva

Id. metr. e trig.

Id. pratica

Id. proj.d. piano

Id. Id.d.spazio

Id. pura

Id. etrig.d.sfera

Gnomonica

Gruppi di trasformaz.

Gravitazione
Interesse e sconto

Logaritmi

Logica matematica

Logismografia

Metrologia

Peso metalli

Problemi di geometr.

Prospettiva

Ragioneria

Id. d. cooper.

Id. industrial.

Ragioniere (pront. d.)

Regolo calcolatore

Repertor. di matemat.

Stereometria

Strumenti metrici

Telemetria

Teoria dei numeri

Id. d. ombre

Termodinamica

Triangolazioni topog. 


\section{Amministrazione pubblica Diritto e Giurisprudenza.}

Assicurazione

Id. esti Diritto costituzionale|Legge s. sanità e sicuAvarie e sinistri mar. Beneficenza

Bonifiche

Catasto

Chimica applicata

Codice del bollo

Id. doganale

Id. civile

Id. proced. civile

Id. commercio

Id. pen.eproc.pen.

Id. di marina

Id. pen. p.l'eserc.

Id. del teatro

Id. d. perito misur. Cod.e leggi us. d'Italia

Computisteria

Conciliatore

Contabilità comunale Id. dello Stato Cooperative rurali

Cooperazione

Curatore dei fallimen. Debito pubblico

Digesto

Diritti e dov. d. cittad.

Diritto amministrativ.

Id. civile

Id. commerciale

Id. Ecclesiastico rezza pubblica

Id. Intern. pubbl. Legge sulle tasse di re-

Id. Id. privato gistro e bollo

Id. penale Legislazione sanitaria

Id. Id. romano Legislazione rurale

Id. romano

Economia politica

Esattore comunale

Estimo dei terreni

Id. rurale

Fognatura cittadina

Giurato (Man. del)

Giurisdizione volont.

Giustizia amministr.

Igiene scolastica

Id. veterinaria

Imposte dirette

Infortuni sul lavoro

Ingegneria legale

Interesse e sconto

Iuoteche

Lavoro donne e fanc.

Legge comunale

Id. sui lav. pubbl.

Id s. ordin. griud.

Id. infort. s.lavoro

Id. 8 propr.letter.

Id.' s. diritti d'aut.

Id. s. priv.industr.
Logismografia

Mandato commerciale Notaio

Ordinam. Stati d'Eur.

Id. Id. f. d'Eur.

Paga giornaliera

Posta

Produz. e commer.vino

Prontuario d. agricolt.

Id. d. ragion.

Proprietario di case

Ragioneria

Ragioneria d. Cooper.

Id. industriale

Ricchezza mobile

Scienza d. finanze

Scritture d'affari

Socialismo

Società di mut. soccor. Id. industriali

Sociologia generale

Statistica

Testamenti

Trasporti e tariffe

Valori pubblici

\section{Archeologia, Belle Arti.}

Amatore oggett. d'arte Anatomia pittorica Antichità greche

Id. priv.d.rom.

Id. pubbl.rom.

Armi antiche

Araldica

Archeol.d. arte greca.

Id. d. arte etr. rom. Architettura

Armi antiche

Arti grafiche fotomec.

Atene

Calligrafia

Colori e pittura
Decoraz. e ind. artist. Disegllo

Id. (Gramm. del) Fiori artificiali

Fotosmaltografia Gioielleria, oreficeria Guida numismatica

Litografia

Luce e colori

Majoliche e porcellane Marmista

Mitologia

Monete greche

Id. romane

Monogrammi
Numismatica

Ornatista

Paleografia

Paleoetnologia

Pittura italiana

Id. ad olio

Prospettiva

Ristauratore dipinti

Scoltura

Storia dell'arte

Teoria d. ombre

Topografia di Roma

Vocabolarietto numis.

Vocabolario araldico 


\section{Storia e Geografia.}

Acque minerali

Alpi

Astronomia (L') n. antico testamento

Atlantest.geog.d.Ital. Id. geog. univers. Cartografia

Climatologia

Commercio (Storia d.) Cosmografia

Cristoforo Colombo

Cronologia

Id. scop.geog. Dizionario alpino

Id. geogratico
Dizionario dei comuni t'Italia

Dizionario biografico

Esercizi geografici

Etnografia

Geografia

Id. ciassica

Id. fisica

Id. commercial.

Geologia

Islanismo

Leggende popolari

Manzoni A.

Mare

Mitologia

Omero
Paleoetnologia

Prealpi bergamasche

Prontuario di geogra f.

Rivoluzione francese

Shakespeare

Sismologia

Statistica

Storia antica

Id. d.artemilitare

Id. del commercio

Id. d'Italia

Id. di Francia

Id. d'Inghilterra

Id. e cronologia

Topografia di Roma

Vulcanisnı

\section{Erudizione, Bibliografia, ecc.}

Amatore oggetti d'art. |Dizionario bibliograf.|Leggende popolari

Id. di maioliche Armi antiche

Atene

Autografi

Bibliogrâia

Bibliotecario

Classificaz. d. scienze

Crittografla

Id. biografico

Id. stenograf.

Id. abbreviat.

Enciclopedia Hoepli

Epigrafia latina

Errori e pregiudizi

Evoluzione (storia d.)

Grafologia
Litografia

Paleoetnologia

Paleografia

Raccoglitore

Stenografia

Stenografo

Tipografia

\section{Filosofia, Pedagogia, Religione.}
Bibbia
Buddismo
Didattica
Diritto ecclesiastico
Estetica
Etica
Evoluzione

Filosotia morale

Giardino infantile

Grafologia

Igiene scolastica

Imitazione Cristo

Logica

Mitologia
Psicologia

Jd. fisiologica

Id. musicale

Protezione animali

Ortofrenia

Religioni dell'India

Sordomuto

\section{Arte militare, Nautica.}

Amatore oggetti d'art Armi antiche

Attrezzatura navale

Avarie e sinistri mar.

Canottaggio

Codice cavalleresco

Costruttore navale

Disegno e costruz.navi

Doveri macchin.naval.
Duellante
Esplodenti
Filonauta
Flotte moderne
Ingegnere navale
Lavori marittimi
Macchinista navale
Marine da guerra
Marino

Meccanica del macchi. nista di bordo

Nautica stimata

Pirotecnia

Scherma

Storia arte militare

Telemetria

Ufficiale 


\section{Letteratura, Linguistica, Filologia.}

A rabo parlato

Arte del dire

Conversaz. Ital.-Ted.

Id. Ital-Fran.

Corrisp. comm.italian.

Id. Id. spagn.

Id. Id. franc.

Crittografia

Dantologia

Dialetti italici

Id. grechi

Dizion. abbrev.latine

Id. bibliografico

id. Eritreo

id. Milanese

Id. Olandese

ld. Tedesco

Id. univ.in 4 ling:

Duttrina pop.in 4 ling.

Enciclopedia Hoepli

Esercizi greci

Id. latini

Id. ditraduzione della gramm. franc.

Esercizi di traduzione della gramm. tedesc.

Filologia classica

Florilegio poet. grevo Fonologia italiana

Id. latina

Fraseologia francese Glottologia

Gravmatica albanese
Grammat. dan.-norv. Id. ebraica

Id. Francese

Id. Galla(Orom.)

Id. Greca

Id. Greca-mod.

Id. Inglese

Id. Italiana

Id. Latina

Id. Olandese

Id. Portoghese-

Brasiliana

Grammat: Rumena

Id. Russa

Id. Slovena

Id. Spagnuola

Id. Svedese

Id. Tedesca

Id. Turca osm.

Leggende popolari

Leøteratura albanese

Id. american.

Id. araba

Id. assira

Id. catalana

Id. dramm.

Id. ebraica

Id. egiziana

Id. francese

Id. greca

Id. indiana

Id. inglese

Id. italiana
Letteratura norveg.

Id. persiana

Id. provenz.

Id. romana

Id. spagnuol.

Id. tedesca

Id. ungheres.

Id. slava

Lingua gotica

Lingue d'A frica

Id. neo-latine

Id. straniere

Hetrica d. greci e rom.

Miorfologia greca

Id. italiana

Omero

Paleografia

Relig. eling. di India

Rettorica

Ritmica italiana

Sanscrito

Shakespeare

sintassi francese

Sintassi latina

Stilistica

Stilistica latina

Tavole divina comm

Tigre

Traduttore tedesco

Verb: greci

Id. latini

Vocabol.lingua Rusea

Volapuk

\section{Musica, Sport.}

Acrobatica e atletica

Alpinisroo

Amatore oggetti d'art.

Armonia

Armi antiche

Antomobilista

Ballo

Biliardo

Cacciatore

Cane (Allevatore del)

Canottaggio

Carito (II)

Cantante
Cavallo

Chitarra

Ciclista

Codice cavalleresco

Dizionario alpino

Id.

filatetico

Dizionario delle corse

Duellante

Filonanta

Ginnastica femminile

Id. maschile

Id. (Storia d.)

Giuochi ginnastici
Giuoco del pallone

Intortuni d. montagna

Lawn-Tennis

Mandolinista

Nuotatore

Pianista

Proverbi sul cavallo

Psicologia musicale

Scacchi

Sicherma

Storia della musica

Strumentazione

Strumenti ad arer 
Elenco completo dei MANUALI HOPPLI disposti in ordine alfabetico per materia.

Abitazione degli animali domestici, del Dott. U. BARPI, L. c. di pag. XVI-372, con 168 incisioni Abitazioni - vedi Fabbricati civili. Abitazioni popolari - vedi Case operaie. Abiti per signora (Confezione di) e l'arte del taglio, compilato da EMrLIA CovA, di pag. VIII-91, con 40 tavole . 3 Abbreviature - vedi Dizionario abbreviature - Dizionario stenografico.

Acetilene (L') di L. CAstellani 2. ${ }^{a}$ ediz. di p. XVI-164 . 2 Acido solforico, Acido nitrico, Solfato sodico, Acido muriatico (Fabbricazione dell'), del Dott. V. VENDER, di pag. VIII-312, con 107 incisioni e molte tabelle. . . 350 Acque (Le) minerali e termali del Regno d'Italia, di Luigi TroLI. Topografia - Analisi - Elenchi - Denominazione delle acque - Malattie per le quali si prescrivono - Comuni in cui scaturiscono - Stabilimenti e loro proprietarî - Acque e fanghi in commercio - Negozianti d'acque minerali, di pag. XXII-552 .

- vedi anche Legislazione delle.

Acrobatica e atletica di A. ZuCCA, di pag. xxx-267, con 100 tavole e 42 incisioni nel testo . . . . . . .650 Acustica - vedi Lnce e suono.

Adulterazioni e falsificazioni (Dizionario delle) degli alimenti, del Dott. Prof. L. GABBA (è in lavoro la $2^{\mathrm{a}}$ ediz.). Agricoltore (Prontuario dell') e dell'ingegnere rurale, dell'Ing. V. NICCOLI, $3^{a}$ edizione riveduta ed ampliata, di pagine XL-500, con 30 incisioni

(li libro deil') Agronomia, agricoltura, industrie agricole del Dott. A. BRUTtrnI, di pag. xx-446 con 303 figure . 350 Agronomia, del Prof. CAREGA DI MURICCE, 3a ediz. riveduta ed ampliata dall'autore, di pag. XII-210. . . . 150 Agronomia e agricoltura moderna, di G. SOLDANI, $2^{a}$ ed. di pag. VIII-416 con 134 incisioni e 2 tav. cromolit. . . 350 Agrumi (Coltivazione, malattie e commercio degli), di A. ALOI, con 22 incisioni e 5 tavole cromolit, pag. XII-238 350 Alcool (Fabbricazione e materie prime), di F. CANTAMESSA di pag. XII-307, con 24 incisioni

Alcool industriale, di G. CIApeTri. Produzione dell'alcole industriale dal punto di vista dell'agricoltnra italiana, applicazione dell'alcole denaturato alla fabbricazione dell'aceto e delle vinacce, alla produzione della forza motrice, al riscaldamento e alla illuminazione con 105 illustrazioni, di pag. XII-262

Algebra complementare, del Prof. S. PINCHERLE:

Parte I. Analisi Algebrica, di pag. VIII-174. . . . 150

Parte II. Teoria delle equazioni, pag. IV-169 con 4 inc. 150 
Algebra elementare, del Prof. S. Pincierle, $8^{\text {a }}$ ediz. di pag. VIU-210 e 2 incisioni . . . . . . . . . . 150 - (Esercizi di), del Prof. S. PINCHERle, di pag. VIII-135, con 2 incisioni. . . . . . . . . . . . 150 Alighieri (Dante) - vedi Dantologia.

Alimentazione, di G. STRAFForello, di pag. VHU-122 . 2 Alimentazione del bestiame, dei Proff. Menozzi E NicCoLI, di pag. XVI-400 con molte tabelle . . . . . 4 Allattamento - vedi Nutrizione del bambino. Alligazione per l'oro o per l'argento - vedi Leghe - Tavole. Alluminio (L'), di C. FoRMENTI, di pag. XXVmr-324 . . . 350 Aloś - vedi Prodotti agricoli.

Alpi (Le), di J. BaLL, trad. di I. Cremona, pag. vi-120 . 150 Alpinismo, di G. BROCHEREL, di pag. VIII-312 . . . . 3 Amalgama - vedi Leghe metalliche.

Amatore (L') di oggetti d'artée e di curiosità, di L. DE MAURI, di 600 pag. adorno di numerose incis. e marche. Contiene le materie seguenti: Pittura - Incisione - Scoltura in avorio - Piccola scoltura - Vetri - Mobili - Smalti - Ventagli - Tabacchiere - Orologi - Vasellame di stagno - Armi ed armature - Dizionario complementare di altri infiniti oggetti d'arte e di curiosità, di pag. XII-580. . . . .6 50 Amianto - vedi Imitazioni.

Anagrammi. - vedi Enimmistica.

Analisi chimica qualitativa di sostanze minerali e organiche e ricerche tossicologiche, ad uso dei laboratori di chimica in genere e in particolare delle Scuole di Farmacia, del Prof.P. E. ALESSANDRI. $2^{\mathrm{s}}$ ediz. intieramente rifatta, di pag. XII384, con 14 inc. numerose tabelle $\Theta 5$ tav. cromolitografiche 5 Analisi di sostanzo alimentari. - vedi Chimica applicata all' Igiene. Analisi delle Urine. - vedi Chimica clinica.

Analisi del vino, ad uso dei chimici e dei legali, del Dott. M. BARTH, traduzione del Prof. E. Comboni, $2^{\text {a }}$ edizione italiana interamente riveduta ed ampliata dal traduttore, di pag. XVI-140, con 8 inc. intercalate nel testo . . . 2 Analisi volumetrica applicata ai prodotti commerciali e industriali, del Prof. P. E. AlesSANDrI, pag. X-342, con inc. 450 Ananas. - vedi Prodotti agricoli.

Anatomia e fisiologia comparate, del Prof. R. BESTA, di pag. VII-218 con 34 incisioni

Anatomia microscopica (Tecnica di), del Prof. D. CARAzZI, di pag. XI-211, con 5 incisioni . 
Anatomia pittorica, del Prof. A Lombardini, $2^{\text {a }}$ ediz. riveduta e ampliata, di pag. virr-168, con 53 inc. . . . 2 Ånatomia topografica, del Dott. Prof. C. FALCone, di pag. $x \vee-395$, con 30 incisioni . . . . . . . . . 3 Anatomia vegetale, del Dottor A. TogNinI, di pagine $\mathrm{XVI}-274$ con 41 incisioni.

Animali da cortile, del Prof. P. BonIzzr, di pag. XrV-238 con 39 incisioni. (Ln $2^{2}$ ediz. è in preparazione).

Animali (Gli) parassiti dell'uomo, del Prof. F. Mercanti, di pag. IV-179, con 33 incisioni . . . . . . . . 150 Antichità greche, del Prof. V. Ivava. (In lavoro).

Antichità private dei romani, del Prof. N. Morescri. $3^{a}$ edizione interamente rifatta del Manuale di W. KoPP, di pag. XVI-181 con 7 incisioni . . . . . . . .

Antichità pubbliche romane di J. G. HUBERT, rifacimento delle antichità romane pubbliche, sacre e militari di W. KoPP, traduzione del Dott. A. WITTGENS, di pag. XIV324 , con 18 figure intercalate nel testo e una pianta. . 3 Antisettici - vedi Medicatura antisettica.

Antologia stenografica, di E. MoLINA (In lavoro), Antropologia, del Prof. G. CANESTRINI, $3^{2}$ ediz., di pag. VI-239 con 21 incisioni. . . . . . . . . . 150 Antropometria, di R. LIVI, di pag. VIIr-237 con 32 incis. 250 Apicoltura, del Prof. G. CANESTRINI, $3^{a}$ ediz. riveduta di

pag. IV-215 con 43 incisioni . . . . . . . . . 2 Appalti - vedi Ingegneria legale.

Arabo parlato ( $\left.\mathrm{L}^{\prime}\right)$ in Egitto, grammatica, frasi, dialoghi e raccolta di oltre 6000 vocaboli del Prof. A. NALLINo. (Nnova ediz. dall'Arabo volgare di DE BTERLICH e DIB KHADDAG) di pag. $\mathrm{XXVIIr-386}$. Araldica (Grammatica), di F. TRIBOLATI, $4^{\text {a }}$ ediz. rifatta da G. DI Crollalanza. (In lavoro).

Aranci - vedi Agrumi.

Archeologia. Arte Greca, del Prof. I. Gentrue (esaurito). È in preparazione una nnova ediz. rifatta del Prof. S. RICCI Archeologia e Storia dell arte italica, etrusca e romana. $3^{a}$ ediz. intier. rifatta. Un vol. di testo con intr. bibliogr. ed appendici sulle nltime scoperte e questioni archeol. di pag. Xxxiv-346 con 96 tav. nel testo a cura del Prof. S. Riccr e un vol. di 79 tav. e in. a cura del Prof. I. GENTILE 750 Architettura (Manuale di) italiana, antica e moderna, di A. MElanI, $4^{2}$ edizione completata ed arricchita, con 136 tav. e 67 incis. intercalate nel testo di pag. XXV-559 750 
Argentatura - vedi Galvanizzazione - Galvanoplastica - Galva-.

L.c. nostegia - Metallocromia - Metalli preziosi - Piccole industr.

Aromatici e Mervini nell'alimentazione. I condimenti, l'alcool (Vino, Birra, Liquori, Rosolii, ecc.). Caffè, Thè Matè, Guarana, Noce di Kola, ecc. - Appendice sull'uso del Tabaceo da fumo e da naso; del Dott. A. VALENTì . . 3 Aritmetica pratica, del Prof. Dott. F. PANIzZA. $2^{\text {a }}$ edizione riveduta, di pag. $v I M-188$. . . . . . . 150

Aritmetica razionale, del Prof. Dott. F. PANIZZA, $4^{2}$ edizione riveduta di pag. Xur-210 . . . . . . . . 150 (Esercizi di), del Prof. Dott. F. PANIzzA, dí p. VIU-150 150 Aritmetica (L') e Geometria dell'operaio, di EzIo GIORLI, di pag. XII-183, con 74 figure

Armi antiche (Guida del raccoglitore e dell'amatore di) di J. GELLI, di pag. VIM-388, con 9 tavole fnori testo, 432 incisioni nel testo e 14 tavole di marche . . . . . 650

Ammonia (Manaale di), del Prof. G. BERNARDI, con prefazione di E. RosSI di pag. XII-288 . . . . . . . 350

Arte del dire (L'), di D. FERRARI, Manuale di rettorica per lo stadente delle Scuole secondarie. $5^{2}$ ediz. corr., (10, 11 e 12 migliaio), pag. XVI-350 e quadri sinottici. . . 150 Arte della memoria (L'), sua storia e teoria (parte scientifica). Mnemotecnia Triforme (parte pratica) del Generale B. PLEBANI, di pag. XXXII-224 con 13 illustr.

Arte mineraria. - vedi Miniere (Coltivazione delle). rte salutare - vedi Memoriale dei Medici pratici.

Arti (Le) grafiche fotomeccaniche, ossia la Eliografia nelle diverse applicaz. (Fotozincotipia, fotozincografia, fotocromolitografia, otolitografia, fotocollografia, fotosilografia, tricromia, fotocollocromia, elioincisione, ecc. secondo i metodi più recenti, con un Dizionarietto tecrico a un cenno storico sulle arti grafiche; $3^{\mathrm{a}}$ ediz. corr. e accrescinta ed in parte rifatta, con molte illustr. di pag. XVI-238 . . . 2 Asfalto (L'), fabbricazione, applicazione, dell'Ing. E. RIGHETTI. con 22 incisioni, di pag. VIII-152.

Assicurazione in generale, di U. GOBBI, di pag. Xui-308. 3 Assicurazione sulla vita, di C. PAGANI, di pag. VI-161.. 150 Assicurazioni (Le) e la stima dei danni nelle aziende rurali, con appendice sui mezzi contro la grandine, del Dr. d. CAPILUPI, di pag. VIII-281, 17 incisioni . . . . 250 Assistenza degl'infermi nell'ospedale ed in famiglia, del Dott. C. Calliano, $2^{2}$ ediz., pag. XXIV-448, 7. tav.

Assistenza dei pazzi nel manicomio e nella famiglia, del Dr.

A. PIERACCTNI, e prefaz. del prof. E. MORSELLI, pag. 250 Astronomia, di J. N. LOCKYER, nuova versione libera con note ed aggiunte del Prof. G. CELORIA, $4^{2}$ ediz. (esaurito, è in lavoro la $5^{a}$ edizione). 
Astronomia (L') nell'antico testamento. di $G$ V. ScHIaPARELLI, di pag. 204 . . . . . . . . . . . . . 150 Astronomia nautica, del prof. G. NACCARI, di pag. XVI320 , con 45 incis. e tav. numeriche.

Atene. Brevi cenni sulla città antica e moderna, seguiti da an saggio di Bibliografia descrittiva e da una Appendice Numismatica, di S. AMBRosoli, con un panorama e una pianta d'Atene, 22 tav. e varie incisioni nel testo. . . 350 Atlante geografico-storico d'italia, del Dott. G. GARoLLo, 24 tav. con pag. VIII-67 di testo e un'appendice. . . Atlante geografico universale, di $R$. KIEPERT, 26 carte con testo. Gli Stati della terra del Dott. G. GAROLLo. $10^{2}$ ediz. aumentata e corretta (dalla $91.000^{\mathrm{a}}$ alla $100.000^{\mathrm{a}}$ copia) pag. VIII-88

Atletica - vedi Acrobatica.

Atmosiera - vedi Igroscopi e igrometxi.

A ttrezzatura, manovra navale, segnalazioni marittime e Dizionarietto di Marina, di F. Imperato, $3^{a}$ edizioue ampliata, di pag. $x \times \Gamma v-643$, con 330 incis. e 28 tav. in cromolit. riproducenti le bandiere maritt. di tutte le naz. 650 Autografi (L'amatore d'), del conte E. BUDAN con 361 facsimili di pag. XIV -426 .

Autografi (Raccolto a raccoglit. di) in Italia di C. VANBIANCHI, di pag. XVI-s76, 102 tav. di facsimili d'ant. e rit. . 650 Automobilista (Nanaale dell') e guida del meccanico conduttore d'automebili. Trattato sulla costruzione dei veicoli semoventi, dedicato agli automobilisti italiani, agli amatori d'antomobilismo in genere, agli inventori, ai dilettanti di meccanica ciclistica, ecc., di G. PEDRETTI, di pag. XXIV -480 , con 181 incisioni . $\cdot \cdot \cdot \cdot \cdot \cdot \cdot \cdot$ quidatore di) del Rag. V. RosseTto. Appendice: Breve dizionario di terminologia tecnico navale e commerciale marittimo inglese-italiano. Ragguaglio dei pesi e misure inglesi con Je italiane. Di pag. Xv-496, con 25 fig. in 6 tav. 550 Âvicoltura - vedi Animali da ortile - Colombi - Pollicoltura. Avyolenamenti - vedi Veleni.

Bachi da seta, del Prof. F. NENCI. 3a ediz. con note ed aggiunte, di pag. XII-300, con 47 incis. e 2 tav. . . . 250

Balistica - vedi Armi antiche - Esplodenti - Pirotecnia - Storia dell'arte militare.

Ballo (Manuale del) di F. GAviNA, di pag. VIII-249, con 92 figure. Contiene: Storia della danza - Balli girati - Cotillon - Danze locali - Feste di ballo - Igiene del ballo. 250 Bambini - vedi Nutrizione dei - Ortofrenia - Terapia.

Barbabietola da zucchero - vedi Zncchero. 
Batteriologia, dei Professori G. e N. CANEstrinI, $2^{\mathrm{a}}$ ediz. in gran parte rifatta, di pag. X-274 con 37 incis. . . . 150 Beneficenza (Manuale della), del Dott. L. CAstralionI, con appendice sulle contabilità delle istituzioni di pubblica beneficenza, del Rag. G. RotA, di pag. XVI-340. • . 350 Eestiame (II) e l'agricoltura in Italia, del Prof. E. ALBERTI, di pag. VIII-312, con 22 zincotipie. . . . . 250 - Vedi Abitazioni di animali - Alimentazione d. bestiame - Cavallo - Coniglicoltura - Igiene veterinaria - Majale - Pollicoltura - Razze bovine - Zoonosi - Zootecnica. Biancheria (Disegno, taglio e confezione di), Mannale teorico pratico di E. BONETTI, con un Dizionario di nomenclatura, $2^{\text {a }}$ edizione riveduta e aumentata, di pag. XVI202 con 50 tavole illustrative \& 5 prospetti . . . . 3 Bibbia (Man. della), di G. M. ZAMPINI, di pag. XII-308 . 250 Bibliografia, di G. OTTINo, $2^{\text {a }}$ edizione riveduta, di pag. IV-166, con 17 incisioni

Bibliotecario (Manuale del), di G. PETzHotor, tradotto sulla $3^{a}$ edizione tedesca, con un'appendice originale di note illustrative, di norme legislative ed amministrative e con un elenco delle pubbliche biblioteche italiane e straniere, per cura di G. BIAGI e G. FUMAGALLI di pagine XX-364-CCXIII. . . . . . . . . . 750

Biliardo (Il ginoco del), di J. GELLI, di pag. $X V-179$, con 79 illustrazioni

Blografia - vedi Cristoforo Colombo - Dantologia - Dizionario biografico - Manzoni - Napoleone I - Omero - Shakespeare. 8iologia animale (Zoologia generale e speciale) per Naturalisti, Medici a Veterinarii del Dott. G. ColtamarinI, di pag. $\mathrm{x}-426$ con 23 tavole. Bollo --vedi Codice del bollo - Leggi registro e bollo.

Bonificazioni (Manuale amministrativo delle) di C. MEzZANOTTI, di pag. XII-294.

Borsa (Operaz. di) - vedi Debito pubblico - Valori pubblici. Boschi - vedi Selvicoltura.

Botanica, del Prof. I. D. HooksR, traduzione del Prof. N.

PEDICINO, $4^{\text {a }}$ ediz., di pag. VIU-134, con 68 incisioni . 150 Botti - vedi Enologia.

Bronzatura - vedi Metallocromia - Galvanostegia.

Eromzo - vedi Leghe metalliche.

Euddismo, di E. PavolinI, di pag. XVI-164 . . . . 150 Burro - vedi Latte - Caseificio.

Bacciatore (Manuale del), di G. FrANCESCHI, 2a edizione

rifatta, di pag. Xurr-315, con 41 incisioni Cacio - vedi Bestiame - Caseificio - Latte, ecc. Caffè - vedi Proảotti agricoli. 
Calcestruzzo (Costruzioni in) ed in cementi armati, di $G$.

VACCHELLI, $2^{2}$ edizione, di pag. XVI-351 con 230 figure 4 Calcí e Cementi (Impiego delle), per l'Ing. L. MazzocceI di pag. XII-212 con 49 incisioni . . . . . . . . 2 Calcolazioni mercantili o bancarie - vedi Conti e Calcoli fatti Interess e sconto - Prontuario del ragioniere - Monete ingl. Calcolo infinitesimale, del Prof. E. PAscAL:

Parte I. Calcolo differenziale, $2^{2}$ edizione riveduta di pag. XII-311 con 10 incisioni

"II. Calcolo integrale, $2^{\text {a }}$ edizione completamente rifatta di pag. VIII-329. . . . . . . 3 III. Calcolo delle variazioni e calcolo delle differenze finite, di pag. XII-300

- Esercizi di calcolo inînitesimale (Calcolo differenziale e integrale), del Prof. E. PASCAL, di pag. $\mathrm{Xx}^{-372}$. di altri apparecchi industriali, di G. BELLUOMINI, di pag. XII-248, con 220 incisioni. . . . . . . . . . Calligrafia (Mannale di). Cenno storico, eifre numeriche, materiale adoperato per la scrittura e metodo d'insegnamento, con 55 tavole di modelli dei principali caratteri conformi ai programmi, del Prof. R. PERcossI, con 38 facsimili di scritture

Calore (II), del Dott. E. JoNES, trad. di U. FORNARI, di pag. VIII-296, con 98 incisioni .

Campicello (II) scolastico. Impianto e coltivazione. Manuale di agricoltura pratiea per i Maestri, dei Dottori E. AZIMONTI e C. CAMPI, di pag. XI-175, con 126 incisioni 150 Cancelilers - vedi Conciliatore.

Candele - vedi Industria stearica.

Cane (Ii) Razze mondiali, allevamento, ammaestramento, malattie con una appendice: I cani della spedizione polare di S. A. R. il Duca degli Abruzzi, di A. VECCHro, $2^{a}$ edizione rifatta, di pag. XVI-442, con 152 inc. e 63 tarole delle quali 12 a colori pa disegni originali dell' autore . 750

Canottagyio (Manuale di), del Cap. G. CropPI, di pagine XXIV-456, con 387 incis. e 91 tav. cromolit. . . . . 750 Cantante (Man. del). di L. MASTRIGLI, di pag. XII-132 . 2 Cantiniere (II). Manuale di vinificazione per nso dei cantinieri, di A. SxRUCCnI, $3^{a}$ edizione riveduta ed aumentata, con 52 incisioni unite al testo, una tabella completa per la riduzione del peso degli spiriti, ed un'Appendice sulla prodnzione e commereio del vino in Italia, di pag. XVI-256

Cant? (Ii) nel suo meccanismo, di P. GueTtA, di p. viII-

258, con 24 incisioni

Carborundum - vedi Imitazioni.

Carburo di caloio - vedi Acetilene 
Cartá (Industria della), dell'Ing. L. SARTORI, di pag. Vu326. con 106 incisioni e 1 tavola. . . . . . . 550 Carte fotografiche, Preparazione e trattameuto, di L. SASSI, di pag. XII. 353

Carte geograñche - vedi Atlante.

Cartografia (Manuale teorico-pratico della), con un sunto della storia della Cartografia, del Prof. E. GELCICE, di pag. VI-257, eon 37 illustrazioni

Casa (La) dell'avvenire, dell' Ing. PEDRINI. Vade mecnm dei costruttori, dei proprietari di case e degli inquilini. Raccolta ordinata di principî d'ingegneria sanitaria, domestica ed urbana, per la costruzione di case igieniche, civili, operaie e rustiche e per la loro manutenzione, di pag. XV-468, con 213 incisioni . . . . . . . . 450

Case coloniche - vedi Economia fabbricati rurali.

Case operaie e abitazioni popolari, di E. MAGRINI, (In lav.). Caseificio, di L. MANETTI, $4^{\text {s }}$ ediz. nuovamente ampliata dal Dr. G. SARTORI di pag. XII-280, eon 49 incisioni . 2 Cataste (Il nuovo) italiano, di E. BRUNI, di pag. VII-346 . 3 -Cavalla (II), del Colonnello C. VolPINI, $3^{a}$ edizione rived. ed ampliata di pag. VI-233 con 48 tavole. . . . 550

Cavi telegrafici sottomarini, Costruzione, immersione, riparazione, dell'Ing. E. JoNA, di pag. XVI-s38, 188 fig. e

1 carta delle comunicazioni telegrafiche sottomarine . . 550 Cediri - vedi Agrumi.

Celerimensura e tavole logaritmiche a quattro decimali del-

l'Ing. F. BorLetTI, di pag. VI-148, con 29 incisioni. . 3 50 Celerimensura (Manuale e tavole di), dell'Ing. G. ORI ANDI, di pag. 1200. con quadro generale d'interpolazioni . . . 18 -.. Calluloide - vedi Imitazioni.

Comentazione - vedi Tempera.

Cementi armati - vedi Calcestruzzo - Calci e cementi.

Ceralacoa - vedi Vernici e lacche.

Ceramiche - vedi Maioliche e porcellane - Fotosmaltografia.

Chimica, del Prof. H. E. RoscoE, 5* edizione rifatta da E. RICCI, di pag. XII-228, con $\$ 7$ incisioni. . . . . . . 150

Chimica agraria, di A. ADUCC0, 2a ediz. di pag. XI-512 $35 \mathrm{C}$ Chimica analitica (Elementi scientifici di), di W. OsTWALD, trad. del Dott. BolIs, di pag. XVI-234. . . . . . 250

Chimica applicata all'igiene. Guida pratica ad nso degli Uficiali sanit.. Medici - Farmacisti - Commerciarti - Laboratori d'igiene, di merciologia, ecc., di P.E. ALESSANDRI, di pag. $\mathrm{XX}-515$, con 49 incisioni e 2 tav. . . . . . . . 550

Chimica clinica, del Prof. R. SUPINo, di pagine XII-202. $.2-$ Chimica legale, (T'ossicologia), di N. VALENTINI, di pachime Xu-243 (Manuale del) e dell'industriale, Raccolta di ta- 
belle, di dati fisici e chimici e di processi d'analisi tecnica, ad uso dei chimici analitici e tecnici, dei direttori di fabbriche, dei fabbricanti di prodotti chimici, degli studenti di chimica, ecc., ecc., del Dottor L. GABBA, $3^{a}$ edizione ampliata, riveduta ed arricchita delle tavole analitiche di H. WILL, di pag. XIX-457, con 12 tavole. . . . . . . 550 Chiromanzia e tatuaggio, note di varietà, ricerche storiche e scientifiche, coll'appendice di un'inchiesta con risposte di Ferrero, Lombroso, Mantegazza, Morselli ed altri di $G$.

L. CERCHIARI, di pag. xx-323. con XXIX tav. e 82 inc. 450 Chirurgia operativa (Man. di), dei Dottori R. STECCEI e A. GARDINI, di pag. VIII-322, con 118 incisioni

Ghisarra (Manuale pratico per lo studio della), di A. PISANI, di pag. XVI-116, con 36 figure e 25 esempi di musica. . . Ciclista, di I. GHersI, $2^{\text {a }}$ ediz. complet. rifatta del "Manuale del Ciclista „ di A. Galante, di pag. 244, 147 inc. . . . 250 Clraiteri - vedi Ingegneria legale.

Civiltà araba (Islamismo) del prof. ITALo PIZZI (in lav.). Classificazione delle scienze, di C. TRIVER0, p. XVI-292 . Climatologia, di L. DE MARCHI, pag. x-204 e 6 carte . . . 150 Cloruro di sodio - vedi Sale.

Codice cavalleresco italiano (Tecnica del dnello), di J. GELLI, 9a ediz. rifatta, di pag. XVI-283 . . . . . 250 Codice del hollo (Il). Nuovo testo unico commentato colle risoluzioni amministrative e le massime di giarisprudenza, ecc., di E. CoRsI, di pag. C-564. . . . . . . . 450

Codice civile del Regno d'Itaiia, accuratamente riscontrato sul testo ufficiale, corredato di richiami $\theta$ coordinato dal Prof. Avv. L. FrancH, $2^{2}$ ediz. di pag. 232 . . 150

Cadice di commercio, accuratamente riscontrato sal testo ifficiale, corredato di richiami e coordinato dal Prof. Avv. L. FRANCHI, 2a ediz. di pag. IV-158. . . . . . . 150

Coulice doganale italiano con commento e note, dell'Avv.

E. BRUNI, di pag. XX-1078 con 4 inc. . . . . . . .

Codice di marina mercantile, secondo il testo wificiale, sorredato di richiami e coordinato dal Prof. Avv. L. FRANCHI, $2^{\text {a }}$ ediz. dii pag. IV-290.

codiso motrico intermazionale - vedi Metrologia.

Coutice penale a di procedura penale, secondo il testo ufficiale, corredato di richiami e coordinato dal Prof. Avv. L. ERANCEI, 2a ediz. di pag. IV-230.

Codice penale per l'esercito e penale militare marittimo, secondo il testo ufficiale, corredato di richiami $\theta$ coordinato dal Prof. Avv. L. FRANCHI, $2^{2}$ ediz. di pag. 179 . . 150 Codice del perito misuratore. Raccolta di norme $\theta$ dati pratici per la misurazione e la valutazione d'ogni lavoro 
edile, prontuario per preventivi, liquidazioni, collaudi, perizie, arbitramenti, degli Ingegn. L. MAZzoccHI e E. MARZORATI, di pag. XuI-498 con 116 illustrazioni . . . . 550

Codice di procedura civile, accuratamente riscontrato sul testo nfficiale, corredato di richiami e coordinato dal Prof. Avv. L. FraNcH, $2^{2}$ ediz. di pag. 167 . . . . . . . 150 Codice sanitario - vedi Legislazione sanitaria.

Codice del teatro (II). Vade-mecnm legale per artisti lirici e drammatici, impresari, capicomici, direttori d'orchesira, direzioni teatrali, agenti teatrali, gli avvocati e per il pabblico, dell'Avv. TABANELI, di pag. XVI-328

Codici e leggi usuali d'ltalia, riscontrati sul testo ufficiale coordinati e annotati dal Prof. Avv. L. FRANCHI, raccolti in quattro grossi volumi legati in pelle flessibile. . . . . 3

Vol. I. Codice civile - di procedura civile - di commercio - penale - procedura penale - della marina mercantile - penale per l'esercito - penale militare marittimo (otto codici) $2^{2}$ edizione, di.pag. viu-1261.

Vol. II. Parte I. Leggi usuali d'Italia. Raccolta coordinata di tatte le leggi speciali più importanti e di più ricorrente ad estesa applicazione in Italia; con annessi decreti e regolamenti e disposte secondo l'ordine alfabetico delle mater

Dalla voce "Abbordi in mare "alla voce "Istruz. pubblica (Legge Casati), „di pag. VIII-1364 a 2 colonne.

Vol. II. Parte II. Dalla voce: Laghi pubblici alla voce: Volture catastali con appen., pag. vur-1369-2982 a 2 col. 12 -

Vol. III. Leggi e convenzioni sui diritti d'antore, raccolta generale delle leggi italiane e straniere e di tatti i trattati e le convenzioni esistenti fra l'Italia ed altri Stati a cura della Società italiana degli autori, $2^{a}$ edizione interamente rifatta dal prof. L. FRANCHI, di pagine VIr-617, legato in tutta pelle flessibile.

Cognac (Fabbricazione del e dello spirito di vino e distil. lazione delle fecce e delle vinacce, di DAL PIAZ, con note del Cav. G. PRAT0, di pag. $\mathrm{X}-168$, con 37 incisioni 2 Coleotteri italiani, del Dott. A. GRIFFINI, (Entomologia I) di pag. XVI-334, con 215 inc.

Collezioni - vedi Amatore d'oggetti d'arte - Amatore di maioliche - Armi antiche - Autografi - Dizionario filatelico.

Calombi domestici e coiombicoltura, del Prof. P. BonIzzr, $2^{a}$ edizione rifatta a cura della Societa Colombifila fiorentina di pag. $x-211$, con 26 figure.

Colorazione dei metalli - vedi Metallocromia.

Colori (La scienza dei) e la pittula di L. GUAITA, p. 248 . 2 -.. Colori a vernici, di G. GoRINI, $3^{3}$ ediz. totalmente rifatta, per c.ra di $G$. APPIANI, di pag. $\mathrm{X}-282$, con 13 incisioni 2 Combustibili - vedi Imitazioni. 
Commedia - vedi Letteratura drammatica.

Commerciante (Manuale del) ad uso della gente di commercio e degli Istituti d'Istruzione commerciale, corredato di oltre 200 moduli, quadri, esempi, tavole dimostrative e prontuari di C. DOMPE, di pag. VI-629. . . . . 550 Commercio, (Storia del) di R. LARICE, di pag. XVI-336 . 3 Commercio - vedi Codice - Corrispondenza commerciale - Compatisteria - Geografia commerciale - Industria zucchero Yandato - Merciologia - Produzione e commercio del vino Ragioneria - Scritture d'affari - Trasporti e Tariffe - Conti fatti - Monete.

Compensazione degli errori con speciale applicazione ai rilievi geodetici, di $\mathrm{F}$. CROTTI, pag. IV-360 Complementi di geometria elementare, del Prof. di $\mathrm{C}$. ALASIA, di pag. $X V-244$, con 117 figure . . . . . 150 Complemento di matemática - (vedi Matematica).

Compositore-sipografo Manuale dell'allievo), di $\mathbf{S}$. LANDI - vedi Tripografia, vol. II.

Compatisteria, del Prof. V. GrTTI:

Vol. I. Compustiteria commerciale, $5^{\mathrm{a}}$ ediz., $\left(9\right.$ e $10^{\circ}$ migliaio) di pag. $1 \nabla-184$. . . . . . . . . . . 150

Vol. II. Computist. finanziaria, $3^{2}$ ediz., pag. VIII-156 . . 150

Camputisteria agraria, del Prof. L. PETRI, seconda edizione rifatta, di pag. VIII-210.

Comuni del Regno d' Italia - vedi Dizionario.

Concta delle pelii ed arti affini, di G. GorINI, 3a edizione interamente rifatta dal Dott. G. B. FRANCESCHI e G. VENTUROLT, di pag. IX-210 .

Conciliatore (Manuale del), deli'Avv. G. PATtaccinI. Guida teorico-pratica con formulario completo pel Conciliatore, Cancelliere, Usciere e Patrocinatore di canse. $4^{2}$ edizione ampliata dall'antore e messa in armonia con l'ultima legge 28 Irglio 1895, di pag. XII-461

Boncimi, del Prof. A. FunARo, $2^{a}$ edizione rinnovata e accresciuta, di pag. XII-266

Concimi fosfatici - vedi Fosfati - Chimica agraria.

Confezione d'abiti - vedi Abiti.

Coniglicoltura pratica, di G. LICCIARDELL, $2^{2}$ ediz., di pag. VII-248, con 53 incisioni e 12 tavole in tricromia . 250 Conservazione delle sostanze alimentari, di G. GorINI, $3^{a}$ edizione intieramente rifatta dai Dott. G. B. FRANCESCHI \& G. VENTUROLI, di pag. VIII-256 . * * • . . 2 -

Consigli pratici - vedi Ricettario domestico - Industriale - Soccorsi d'argenza.

Contabilita comunale, secondo le nnove disposizioni legislative e regolamentari (Testo unico 10 febbraio 1889 e R.

Decr. 6 luglio 1890), del Prof. A. DE BRUN, pag. VIII-186 . 150

Contabilità domestica, Nozioni amministrativo-contabili ad aso delle famiglie e delle scrole femminili, del Rag. 0. BERGAMASCHI, di pag. XVI-186. 
Contabilità generale delio Stato, dell'Avv. E. BRuNI, $2^{2}$ edizione rifatta, pag. XVI-420.

Contabilità delle istituzioni di p. beneficenza - vedi Beneficenza.

Conti e calcoli fatti, dell'Ing. I. GHERSI, 93 tabelle e istruzioni pratiche sul modo di usarle. (Misure, Pesi, Monete, Termometro, Gas e Vapori, Areometri, Alcoolometri, Soluzioni zaccherine, Pesi specifici, Legnami, Carboni, Metalli, Divisioni del tempo, Paga giornaliera, Interessi e Annualità, Rendita, Potenze e Radici, Poligoni e Poliedri regolari, Sfera, Circolo, Divisione della circonf., Pendenza, pag. 204. . Contratti agrari - vedi Mezzeria.

Conyersazione italiana e tedesca (Manuale di), ossia guida completa per chinnque voglia esprimersi con proprietà e speditezza in ambe le lingue, e per servire di vade mecum ai viaggiatori, di A. FIORI, $8^{\text {a }}$ edizione rifatta da G. CATTANEO, pag. XIV -400

Conversazione italiana-francese - V. Fraseologia

Cooperative rurali, di credito, di lavoro, di prodnzione, di assicurazione, di mutuo soccorso, di consumo, di acquisto di raterie prime, di vendita di prodotti agrari. Scopo, costitazione, norme giuridiche, tecniche, amministrative, compntistiche, di V. NICCOLI, pag. VIII-362.

Cooperazione nella spciologia e nella legislazione, ui F. VIRGILI, pag. Xu-228.

Correnti elettriche alternate semplici, bifasi e trifasi. Manuale pratico per lo studio, costruzione ed esercizio degli impianti elettriei, dell'Ing. A. MARro, di pag. XIV615-LXIV, con 218 incisioni e 46 tabelle.

Corrispondenza commerciale noliglotta, di G. FRISONI, compilata su di un piano speciale nelle lingue ital., francese, tedesca, inglese e spagnuola, di cui ciascuna forma in sè stessa l'originale e le altre ne sono la traduz. o la chiave:

L. - PARTE ITALIANA: Manuale di Corrispondenza Commercialo italiana corredato di facsimili dei vari documenti di pratica giornaliera, seguito da un Glossaro delle principali voci ed espressioni attinenti al Commercio, agli Affari marittimi, alle Operazioni bancarie ed alla Borsa, ad uso delle scuole, dei Banchieri, Negozianti ed Industriali di qualunque nazione, che desiderano abilitarsi alla moderna terminologia e nella corretta fraseolagia mercantile Italiana, di pag. $\mathrm{xx}-444$

II. - PARTE SPAGNUOLA: iranual do Correspondencia Comercial Espanola, accompañado de facsimiles de los varios documentos de aso cotidiano, seguido de un Drccronario Español-Italiano que contiene las principales voces empleados en los Negocios mercantiles y maritimos y los terminos más importantes del Banco, de la Contabilidad y de la Bolsa, compuesto para uso de las Escuelas, de los Banqueros, Negociante é industriales de cralquiera nación que desean habilitarse en la moderna terminologia y en la corriente fraser logia mercantil española,p. xx-440. 
III. - PARTE FRANCESE: Manuel de Correspondance commerciale française, accompagné des fac-similes des differents documents d'nsage quotidien, snivi d'un Dictionnaire commercial françaisitalien contenent les principales expressions du langage mercantil et maritimes et les termes les plus importants de Banque de comptabilité, de Bourso et De Chemins de Fer, à l'usage des Ecoles, des Banquiers, des Négociants et Industriels qui derivent se perfectionner dans la terminologie moderne et dans la phraséologie mercantile française de nos jours, di pag. vxI-446

IV.-PARTE INGLESE: A Manual of english Commercial correspondence, including specimens and forms of different documents of daily use, followed by an English and Italian Mercantile Dictionary containing the principal expressions employed in trade, commerce and shipping concerns, and the most important terms of Bank, Book-Keeiping, Stock-Exchange and Railway for the use of students, bankers, merchants and manufacturers who wish to perfect themselves in the terminology and phraseology as actually in current use with english business men, p. xvI-448 V. - PARTE TEDESCA (in lavoro).

Corse (Le), con un Dizionario delle voci più in uso, di G. FRANCESCEI, di pag. XII-305 - cं

Cosmografia. Uno sguardo all'Universo, di B. M. LA LETA, pag. XII-197, con 11 incisioni e 3 tavole. . . . . . 150 Costisuzione degli Stati - vedi Diritti e doveri - Ordinam.

Costruttore navale (Manuale del), di G. RossI, pag. XVI517 , con 231 fig. intercalate nel testo e 65 tabelle.

Costruzioni - vedi Fabbricati rurali.

Cotoni - vedi Prodotti agricoli.

Gremore di tartaro - vedi Distillazione.

Crista!lo - vede Specchi.

Cristallografia geometrica, fisica e chimica, applicata ai minerali, di E. SANSONI, D. XVI-367, 284 inc. nel testo : 3 Cristo - vedi Imitazione di Cristo.

Cristoforo Colombo, di V. BELLro, pag. IV-136 e 10 incis. . 150 Crittogame - vedi Funghi - Malattie crittogamiche - Tartufi.

Crittografia (La) diplomatica, militare e commerciale, ossia l'arte di cifrare e decifrare le corrispondenze segrete. Saggio del conte L. GIOPPI, pag. 177

Cronologia delle Scoperte e delle esplorazioni geografiche dall'anno 1492 a tutto il secolo $X X$ del Prof. $L$. HUGUES, di pag. VIII-487

Cronologia - vedi Storia e cronologia.

Cubatura dei legnami (Prontuario per la), di G. BELLdoNINI, $4^{\text {a }}$ ediz. corretta ed accresciuta, pag. 220 .

Guoio - vedi Concia delle pelli - Imitazioni.

Curatore dei fallimenti (Manuale teorico-pratico del) Commissario gindiziale nel concordato preventivo e procedura dei piccoli fallimenti dell'Arv. Mourna (ln lavoro).

Curiosita - vedi Amatore di oggetti d'arte - Maioliche e porcellane - Armi antiche - Autografi. 
Curve circolari e raccordi. Mannale pratico per il tracciamento delle curve in qualunque sistema e in qualsiasi caso particolare nelle ferrovie, strade e canali e per il computo generali dei raccordi circolari con speciali applicazioni al tracciamento dei raddoppi del Binario delle derivazioni $e$ degli scambi ferroviari (In sostituzione del manuale del KRÖNHKE), di C. FERRARIO, pag. XI-264, con 94 incis. . . 350 Dantologia, del Dott. G. A. ScARTAzzinI, $2^{\text {a }}$ edizione. Vita e Opere di Dante Alighieri, pag. VI- -408

Danze - vedi Ballo.

Datteri - vedi Prodotti agricoli.

Debito (Il) pubblico italiano. Regole e modiper le operazioni sui titoli che lo rappresentano, di F. AzzoNI, pag. VIII-376 3 Decorazione dei metalli - vedi Metallocromia.

Decorazioni del vetro - vedi Specchi - Fotosmaltologia.

Decorazioni e industrie artistiche, dell'Architetto A. MELANI, 2 volumi, pag $\mathrm{xX}-460$, con 118 incisioni. Denti - vedi Igiene della bocca.

Determinanti e applicazioni, di E. PASCAL, pag. vI-330 . 3 Diagnostica - vedi semeiotica.

Dialetti italici. Grammatica, iscrizione, versione e lessico, di O. NAZARI, pagine XVI-364 . . . . . . . . 3 Dialetti letterari greci (epico, neo-ionico, dorico, eolico), del Pof. G. BonINo, pag. XXXII-214 . . . . . . 150

Didattica per gli alunni delle Scnole normali e pei maestri elementari, del Pof. G. SoLr, pag. vIr-314 . . . . . 150 Digesto (I1), del Prof. G. FERRINI, pag. IV-134 . . . . . 150 Dilsttanti di pittura - vedi Pittura ad olio.

Dinamica elementare, di G. CATTANEO, p. VIII-146, 25 fig. 150 Dinamite - vedi Esplodenti.

Diritti e doveri dei cittadini, secondo le Istituzioni dello Stato, per aso delle pubbliche Scuole, del Prof. D. MAFFIOL., $10^{\text {a }}$ edizione, (dal 26 al $30^{\circ}$ migliaio) con una appendice sul Codice penale, pag. XVI-229

Diritti d"Autore - vedi Leggi sui.

Diritto amministrativo, giusta i programmi governativi ad uso d. Istituti tecnici, di G. LORIS, $5^{2}$ ed. pag. $x X-4743-$ Diritto civile (Compendio di), del Prof. G. LoRIs, giusta i programmi governativi ad uso degli Istituti tecnici, $2^{\mathrm{a}}$ ediz. riveduta, corretta ed ampliata, pag. XVI-385. . . . . . 3 -

Diritto civile italiano, di C. ALBICINI, p. VIII-128. . . 150

Diritto commerciale italiano, del Prof. E. VIDARI, $2^{\text {a }}$ edizione diligentemente riveduta, pag. $\mathrm{x}-448$. . . . . . 3 -

Diritto comunale e provinciale - vedi Contabilità comunale - Diritto amministrativo - Legge comunale.

Diritto cestituzionale, dell'Avv. Prof. F. P. ContuzzI, 2a edizione, pag. XVI-370

Diritto ecclesiastico, vigente in Italia. $2^{a}$ ediz. riveduta ed ampliata di G. OLMO, pag. XVI-483 
Diritto internazionale privato, dell'Avv. Prof. F. P. ConTUZZI, pagine XVI-32:

Diritto internazionale pubblico, dell'Avv. Prof. F. P. CoNTUZZI, pagine XII-3220.

Diritte perale, dell'Avv. A. SToppato, $2^{\mathrm{a}}$ ediz., (in lavoro) Diritto penale romano, di C. FERRINI, pag. VIII-360. . . Diritto romano, di C. FERRINI, $2^{2}$ ediz. rif., pag. XVI-178 . Disegnatore meccanico e nozioni tecniche generali di Aritmotica, Geometria, Algebra, Prospettiva, Resistenza dei materiali, Apparecchi idraulici, Macchine semplici ed a vapore, Prepulsori, ecc. per V. GOFFI, $3^{a}$ ed. riv., corretta e grandemente ampliata, pag. XIV-552, con 477 figure.

Disegne. I principii del Disegno, del Prof. C. Borto, $4^{2}$ edizione, pag. IV-206, con 61 silografie.

Disegne (Grammatica del). Metodo pratico per imparare il disegno, di E. RoNCHETTI, di pag. VI-190, con 34 figure, 62 schizzi intercalati nel testo e un atlante a parte con 45 lavagnette, 27 foglietti e 34 tavole. (Indivisibili) . . 750 Disegno assonometrico, del Prof. P. PAOLONI, pag. IV-122, con 21 tavole e 23 figure nel testo

Disegmo geometrico, del Prof. A. ANTuLLI, $2^{a}$ ed., pag. VIII88 , con 6 figure nel testo e 27 tavole litografiche.

Disegno, Teoria e Costruzione delle Navi, ad uso dei Progettisti e Costruttori di Navi - Capi tecnici, Assistenti e Disegnatori navali - Capi operai carpentieri - Alunni d'Istituti Nautici, di E. GIorLI, pag. VIII-238, con 310 incisioni .
isegno industriale, di E. Grorul. Corso regolare di disegno geometrico e delle proiezioni. Degli sviluppi delle superfici dei solidi. Della costrizione dei principali organi delle macehine. Macchine utensili. $3^{a}$ ediz., pag. VIII-192, con 300 problemi risolti e 348 fircure

Disegno di proiezioni ortogonalí, del Prof. D. Land, di pagine VIII-152, con 192 incisioni

Disegno topografico, del Capitano G. BERTELLI, $2^{\text {a }}$ ediz., pagine. VI-137, con 12 tavole e 10 incisioni . $\cdot$. Disinfezione (La pratica della) pubblica e privata per i Dot tori P. E. ALESSANDRI e L. PIZZINI, $2^{2}$ edizione, pag. VII258 , con 29 incisioni .

Distillazione del legno (Lavorazione dei prodotti della). Acetone, Alcool metilico, Aldeide formica, Cloroformio, Acido acetico, Acetato di piombo, Acetato di sodio. Industrie elattrochimiche. Ossidi di piombo, Minio, Biacca, Soda canstica, Clorati, Cromati, dell' Ing. F. VILLANI, di pagine XIV-312.

Distillazione delie Vinacce, e delle frutta fermentate. Fabbricazione razionale del Cognac. Estrazione del cremore di Tartaro ed utilizzazione di tutti i resi. 
dui della distillazione, di M. DA PONrE, $2^{\text {a }}$ edizione rifatta, contenenti le leggi italiane sugli spiriti e la legge Austro-Ungarica, pag. XII-375, con 68 incisioni . . . . 350 Ditteri italiani, di PAOLO LIOY (Entomologia III), pag. VII-356, con 227 incisioni

Dizionario alpino italiano. Parte $1^{\text {a: }}$ Vette e valichi italiani, dell'Ing. E. BIGNAMI-SORMANI. -- Parte 2a: Valli lomỏarde e limitrofe alla Lombardia, dell'Ing. C. ScoLARI, pag. XXII-310.

Dizionario di abbreviature latine ed italiane usate nelle carte e codici specialmente del Medio Evo, riprodotte con oltre 13000 segni incisi, agginntovi an prontuario di Sigle Epigrafiche. I nonogrammi, la namerizzazione romana ed arabica e i segni iudicanti monete, pesi, misure, ecc., per cura di ADRIANO CAPPELLI, Archivista-Paleografo presso il R. Archivio di Stato in Milano, pagine Lxu- 433 ; con elegante legatura in cromo . . . . . . 750 Dizionario bibliografico, di C. ARLIA, pag. 100 . . . . 150 Dizionario Biografico Universale, del Professor Dottor G. GAROLLO. (In Lavoro).

Dizionario dei comuni del Regno d'Italia, secondo il Censimento del 10 febbraio 1901, compilato da B. SANTI, di pag. XLVI-175.

Dizionario Eritreo (Piccolo) italiano-Arabo-Amarico, raccolta dei vocaboli più usuali nelle principali lingue parlate nella Colonia Eritrea, di A. ALLORI, pag. XXXIII-203

Dizionario filatelico, per il raccoglitore di francobolli con introduzione storica e bibliografica, di J. GELLI, $2^{\text {a }}$ ediz., con Appendice 1898-99, pag. LXIII-164 . . . . . . .460

Dizionario fotografico pei dilettanti e professionisti, con oltre 1500 voci in 4 lingue, 500 sinonimi e 600 formule di L. GIOPPI, pag. VIII-600, 95 incisioni e 10 tavole. . . . 750 Dizionario geografica universale, del Prof. Dott. G. GAROLLO. $4^{\mathrm{a}}$ edizione del tutto rifatta e molto ampliata, di pagine XII-1451

Dizionario gotico - vedi Lingua gotica.

Dizionario italiano-olandese e olandese-italiano, di A. NUYENS, in-16, di pag. XI-948.

Dizionario milanese-italiano e repertorio italiano-milanese, di CLETTO ARRIGHI, pag. 912, a 2 colonne, 2a ediz. 850 Dizionario Humismatico - vedi Vocabolarietto numismatico.

Dizionario rumeno - vedi تีrammatica rumena.

Dizionario stenografico. Sigle e abbreviature del sistema fabelsberger-Noe, di A. SChIAVEnATo, pag. XVI-156 . . 150 
Dizionario tascabile (Nuovo) italiano-tedesco e tedescoL.c. italiano, compilato sui migliori vocabolari moderni e provvisto d'un'aceurata accentuazione per la pronuncia dell'italiano, di A. FiorI, $3^{\text {a }}$ edizione, pag. 798, completamente rifatta dal Prof. G. Cattaneo . . . . . . . . . . . 350 Dizionario tecnico in quattro lingue dell'Ing. E. WEBBER, 4 volumi:

Vol. I. Italiano-Tedesco-Francese-Inglese, $2^{\text {a }}$ ediz. completamente riveduta e aumentata di cirea 2000 termini tecnici, pag. XII-553 . . . . . . . 6 --

Vol, Ir. Deutsch-Italienisch-Französisch-Englisch, (esanrito, è in lavoro la $2^{\mathrm{a}}$ edizione).

Vol. III. Français-Italien-Allemand-Anglais, pag. 509 . . 4 -

Vol. IV. Englisch-Italian-German-French, pag. 659.

Dizionario tecnico-navale e commerciale marittimo inglese-italiano.

- Vedi Avarie e sinistri marittimi.

Dizionario (Piccolo) dei termini delle corse, di G. VOLPINI, di pagine 47 . (Esaurito).

Dizionario turco - vedi Grammatica turca.

Dizionario universale delle lingue italiana, tedesca inglese e francese, disposte in unico alfabeto, 1 rolume di pag. 1200 a 2 colonne

Dizionario Volapük - vedi Volapük.

Dogane - vedi Codice doganale - Trasporti e tariffe.

Doratura - vedi Galvanizzaz. - Galvanostegia - Metallocromia.

Dottrina popolare, in 4 lingue, (Italiana, Francese, Inglese

$\theta$ Tedesca). Motti popolari, frasi commerciali e proverbi, raccolti da G. SESSA, $2^{\text {a }}$ edizione, pag. IV-112 . . . . .

a vapore marina ad tiso deị maechinista navali e degli Istitrti nantici, di M. LIGNAROLO, pag. XVI-303 . . . . . 250 Dramns - vedi Letteratura drammatica.

Duellante (Manuale del) in appendice al Codice cavalleresco,

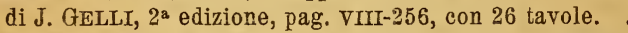

Ebanista - vedi Falegname - Modellatore mecc. - Operaio.

Educazione dei bambini - védi Ortofrenia - Sordomuti.

Economia matematica (Introduzione alla), dei Prof. F. VIRGILII e C. GARIBALDI, pag. XII-210, con 19 incisioni . . 150 Economia polisica, del Prof. W. S. JEvons, traduzione del Prof. L. COSSA, $4^{2}$ ediz. riveduta, pag. XVI-179. . . . . 150 Edilizia - vedi Fabbric. civili - Ingegneria civ. - Ingegn. leg. Elettricità, del Prof. FleEming Jenkin, traduz. del Prof. R. FERRINI, 3a ediz. rived., pag. XII-237, con 40 incisioni . 150 Elettrochimica (Prime nozioni elementari di), del Professor A. CoSSA, pagine VIIr-104, con 10 incisioni. . . . . 150 
Elettrotecnica (Manuale di, di GRAWINKEL-STRECKER, traduzione italiana dell'Ing. FLAvIo DESSY, pagine XVI-816, con 346 figure. . . . . . . . . . . . . 950 Elettrochimiche (Industrie) - vedi Distillazione del legno.

Ematologia - vedi Malattie del sangue.

Embriologia e morfologia generale, del Prof. G. CATTANEO, pag. $X-242$, con 71 incisioni. . . . . . . 180 Enciclopedia del giurista - vedi Codici e leggi usuali d'Italia.

Enciclopedia Hoepli (Piccola), in 2 grossi vol. di 3375 pag. di 2 col. per ogni pag., con Appendice (146740 voci) . . . . 20 -

Energia fisica, del Prof. R. FERRINI, pag. VIII-187, con 47 incisioni. $2^{\mathrm{a}}$ edizione interamente rifatta . . . . . 150

Enimmistica. Guida per comporre e per spiegare Enimmi, Sciarade, Anagrammi, Logogrifi, Rebus, ecc., di D. TOLOSANI (Bajardo), pag. XII-516, con 29 illustr. e molti esempi 650

Enologia, precetti ad uso degli enologi italiani, del Professor O. OTTAVI, $4^{2}$ edizione interamente rifatta da A. STRUCCHI, con una Appendice sul metodo della Botte mnitaria pei calcoli relativi alle botti circolari, dell'Ing. agr. R. BASSI, pag. XVI-304, con 38 incisioni

Enologia domestica, di R. SERNAGIotTo, pag. VIII-233. 2 Entomologia di A. Griffini E P. Lioy, 4 volumi (vedi Coleottori - Ditteri - Lepidotteri - Imenotteri).

Epigrafia latina. Trattato elementare con esercizi pratici $\theta$ facsimili, con 65 tav. del Prof. S. RICCI. pag. XXXII-148 . 650 - vedi Dizionario di abbreviature latine.

Epilessia, Eziologia, Patogenesi, Cura, Dr. P. PrNI, p. X-277 250 Eritrea (L') dalle sue origini a tutto l'anno 1901. Appunti cronistorici con annessi 1 carta ed 1 schizzo, un' appendice di note geografiche e statistiche e di cenni sul $\mathrm{Be}$ nadir e sui viaggi d'esploraz, di B. MeLLI, di pag. XII-164

Eritrea - vedi Arabo parlato - Dizionario eritreo, - Grammatica galla - Lingue d'Africa - Prodotti agricoli del Tropico - Tigrè italiano.

Eprori e pregiudizi volgari, confutati colla scorta della scienza e del raziocinio da G. STRAFFORELLO, $2^{\text {a }}$ edizioue accrescinta, pag. XII-196

Esame degli infermi - vedi Semeiotica.

Esattore comunale (Manuale dell'), ad uso anche dei Ricevitori provinciali, Messi esattoriali, Prefetti, Intendenti di finanza, Agenti imposte, Sindaci e Segretari dei Comuni, Arvocati, Ingegneri, Ragionieri, Notai e Contribuenti, del Rag. R. MATNARDI, $2^{2}$ ediz. rived. e ampl., pag. XVI-480 . 550

Esercizi geografici e quesiti, sull'Atlante geografico universale di R. Kiepert, di L. HuGUES, $3^{2}$ ediz. rifatta di pagine VIII-208 : . . . . . . . . . . . . 150

Esercizi sulla geometria elementare, del Prof. S. PINCHERLE, pag. VIII-130, con 50 incisioni . . . . . . 150 
Esercizi greci, per la $4^{2}$ classe ginnasiale in correlazione alle Nozioni elemen. di lingua greca, del Proi. V. INAkna: del Prof. A. V. BisConti, 2a ediz. rifatta, di p. XXVI-234 . a Esercizi latini con regole (Morfologia generale), del Prof.

P. E. CERETI, pag. XII-332. . . . . . . . . . . . 150 Esercizi di stenografia - vedi Stenografia.

Esercizi di traduzione a complemento della grammatica francese, del Prof. G. PRAT, pag. VI-183 . . . . . . 150 Esercizi di traduzione con vocabolario a complemento della Grammatica tedesca, di G. ADLER, 2a ed., p. VIII-234. 150 Esercizi ed applicazione di Trigonometria piana, con 400 esercizi e problemi proposti dal Prof. C. ALASIA, pag. XVI-292, con 30 incisioni

Esploderti e modo di fabbricarli, di R. MoLINA, p. XX-300 250 Espropriazione - vedi Ingegneria legale.

Essenze - vedi Profumiere - Liquorista - Ricettario ind.

Estetica, del prof. M. PrLo, di pag. xx-260 . . . . . 150

Estime dei terreni. Garanzia dei prestiti ipotecari e della equa ripartizione dei terreni, dell'Ing. P. FILIPPINI, pag. XVI-328, con 3 incisioni.

Estimo rurale, del Prof. CAREGA DI MURICCE, pag. VI-164. 2 Etica, (Elementi di) del Prof. G. VIDARI, di pag. XVI-3̊4 . 3 Etnografia, di B. MalfatTI, 2a ediz. inter. rifusa, p. VI-200, 150 Euclide (L') emendato del P. G. SACCHERI, traduzione e note di G. BOCCARDINI di pag. XXIV-126 con 55 inc. . 150

Evoluzione (Storia dell'), del Prof. CARLo FENIzIA, con brove saggio di Bibliografia evolnzionistica, pag. XIV-389 . . 3 -

Fabbricati civili di abitazione, dell'Ing. C. LEVI, 2a ediz. rifatta, con 207 incis., e i Capitolati d'oneri approvati dalle principalí città d'Italia, pag. XVI-4.12

Fabbricati rurali (Costruzione ed economia dei). $2^{2}$ edizione rifatta dall" "Economia dei fabbricati rurali ", di V. NICCOLI, di pag. XVI-335, con 125 figure . . . . . . 350

Fabbro - v. Aritmetica dell'operaio - Fonditore - Meccanico Operaio - Tornitore.

Fabbro-ferraio (Manuale pralico del), di G. BELLuominI, opera necessaria ed indispensabile ai fabbri fucinatori, agli aggiustatori meccanici, armajuoli, carrozzieri, carradori, calderai, coltellinai, fumisti, costruttori di strumenti metrici, di serrature, di arnesi rurali, di ferramenti in genere ed a tutti quelli che si dilettano nei lavori in ferro ed in acciaio, di pag. VIII-242, con 224 incisioni . . . 250

Falegname ed ebanista. Natura dei legnami, maniera di conservarli, prepararli, colorirli e verniciarli, loro cubatura, di G. BELLUOMINI, 3ª ed. di pag. XII-224, con 104 incisioni 
Fallimenti (Vedi Curatore di).

Fanciutli - (idioti, imbecilli, tardivi, ecc.) v. Ortofremia.

Farfalio - vedi Lepidotteri.

Farmacista (Manuale del), del Prof. P. E. ALESSANDRI, 2a edizione interamente rifatta e aumentata, corredata di tutti i nuovi medicamenti in uso nella terapentica, loro proprietà, caratteri, altexazioni, falsificazioni, usi, dosi, ecc., pag. xVI731 , con 142 tavole e 82 incisioni

Farmacoterapia e formulario, del Dott, P. PICcrininI, di pag. VIII-382

Ferrovie - vedi Codice doganale - Curve - Ingegneria legalo Macchin. e Fuochista - Trasporti e tariffe.

Filatelia - vcdi Dizionario filatelico.

Filatura. Manuale di filatura, tessitura e lavorazione meceanica delle fibre tessili, di E. GROTHE, traduzione sull'ultima. tedesca, pag. VIII-414, con 105 incisioni . . . . . . 5 -

Filologia classica, greca e latina, del Prof. V. INAMA, di pag. XII-195 . . . . . . . . . . . . 150

Filonauta, Quadro generale di navigazione da diporto e consigli ai principianti, con un Vocabolorio tecnico più in uso nel panfiliamento, del Cap. G. OLIVARI, pag. XVI-286 . . 250

Filosofia morale, del Prof. L. FRIso, $2^{a}$ edizione riveduta ed aumentata, di pag. XVI-350 .

Fillossera e le principali malattio crittogamichs della vite con speciale riguardo ai mezzi di difesa, del Dott. V. PEGLION, pag. VII-302, con 39 incisioni .

Filugello - vedi Bachi da seta.

Fiori artificiali, Manuale del fiorista, di O. BALLERINI, pag. XVI-278, con 144 incisioni, e 1 tav. a 36 colori . . . 350

- vedi anche Pomologia artificiale.

Fisica, del Prof. O. MURANI, con 243 incisioni e 3 tavole, 6s edizione, completamente rifatta del Manuale di Fisica di BALFOUR STEWART pag. XVI-411 . . . . . . . . 2 -

Fisica cristallografica, W. VorGT, trad. A. SELLA. (In lav.). Fisiologia, di Foster, traduzione del Prof. G. ALBINI, 3a edizione, pag. XII-158, con 18 incisioni . . . . . . 150

Flsiologia comparata - vedi Anatomia.

Fisiologia vegetale, del Dott. LuIGI MonTEMartini, pag. XVI-230, con 68 incisioni . . . . . . . . . 150

Floricoltura (Manuale di), di C. M. Fratelli RODA, $3^{\text {a }}$ ed. riveduta ed ampliata da G. RODA, pag. vIII-262, con 98 inc. 250 Florilegio poetico greco, del Prof. V. Inama. (In lavoro).

Flotte moderne (Le) 1896-1900, di E. BUCCI DI SANTAFIoRA. Complemento del Manuale del Marino, del C. DE AMEZAGA, pagine IV-204 
Fognatura cittadina, dell'Ing. D. SPATAR0, pag. X-684, con 220 figure e 1 tavola in litografia

Fognatura domestica, dell'Ing. A. CERUTTI, pag. VII-421, con 200 incisioni

Fonditore in tutti i metalli (Manuale del), di G. BeLLUOMLNI, $2^{\text {a }}$ edizione, pag. VIIr-150, con 41 incisioni .

Fonologia italiana, di L. STOPPATo, pag. vIщ-102. . . . 150

Fonologia latina, del Prof. S. Corsolr, pag. 208 . . . . 150

Foreste - vcdi Ingegneria legale - Selvicoltura.

Formaggio - vedi Caseificio - Latte, burro e cacio.

Formole e tavole per il calcolo delle risvolte ad arco circolare, adattate alla divisione centesimale ad uso degli ingegneri, di F. BoRLETTI, di p. XII-69, legato. . . 250

Formulario scolastico di matematica elementare (aritmetica, algebra, geometria, trigonometria), di HI. A. RossotTr, di pag. XVI-192

Fosfati, perfosfati e concimi fosfatici. Fabbricazione ed analisi del Prof. A. MrNozZI, di pag. Xı-301 con 48 inc. 350 Fotocaichi - vedi Arti grafiche - Chimica fotografica - Fotografia industriale - Processi fotomeccanici.

Fotocoilografia - vedi Processi fotomeccanici.

Fitocrematografia (La), del Dott. L. SASSI, pag. XXI-138, con 19 incisioni

Fotografia industriale (La), fotocalchi economici per la rjproduzione di disegni, piani, sarte, musica, negative foto grafiche, ecc., del Dott. LUIGI GIOPPI, pag. VIII-208, con 12 incisioni e 5 tavole fuori testo.

Fotografia ortocromatica, del Dott. C. BoNACINI, pagine IVI-277, con incisioni e 5 tavole. . . . . . . . 350

Fotografia poi dilettanti. (Come dipinge il sole), di $G$. MUEFONE, $5^{\mathrm{a}}$ edizione rifatta ed ampliata, pag. $\mathrm{XX}-383$, con 99 incisioni e 11 tavole.

Fotogrammetria, Fototopografia praticata in Italia e applizione della fotogrammetria all'idrografia, dell'Ing. P. PAGANINI. pag. XVI-288, con 56 figure e 4 tavole.

Fotolitografia - vedi Arti grafiche - Processi fotomecc.

Fotosmaltografia (La), applicata alla decorazione industriale delle ceramiche e dei vetri, di A. MonTAGNA, pag. VIII-200, con 16 incisioni nel testo

Fototerapia e radioterapia - vedi Lnce e salute.

Folotipografia - vedi Arti grafiche - Processi fotomecc.

Fragole vedi Frutta minori.

Francia - vedi Storia della Francia.

Francoholli - vedi Dizionario flatelico.

Fraseologia francese-italiana, di E. BAROSCHI SORESINI, pag. VIII-262

Fraseologia iialiana-tedesca - vedi Conversazione - Dottrina popolare.

Frenastenia - vedi Ortofrenia. 
Frumento (II), (come si coltiva o si dovrebbe coltivare in Italia) di E. AzrmonTr, $2^{a}$ edizione completamente rifatta del Manuale "Frumento e mais "di G. CANTonI, di pagine XVI-276

Frutta minori. Fragole, poponi, ribes, uva spina e lamponi, del Prof. A. PuCCI, pag. VIII-193, con 96 incisioni . . 250 Frutta fermentate - vedi Distillazione

Frutticoltura, del Prof. Dott. D. Tamaro, 3a edizione, di pag. XVUI-219, con 81 incisioni . . . . . . . . $2-$ Frutti artificiali - vedi Pomologia artificiale.

Fulmini e parafulmini, del Dott. Prof. CANestrint, pag. VuI-166, con 6 incisioni . . . . . . . . . . 2 -

Funghi mangerecci e funghi velenosi, del Dott. F. CAVARA, di pag. XVI-192, con 43 tavole e 11 incisioni . . 450 Funzioni analitiche (Teoria delle), di G. VIVANTI, pagine vIII-432 (volume doppio).

Funzioni ellittiche, del Prof. E. PASCAL, pag. 240. 150 Fuochista - vedi Macchinista e fuochista.

Fuochi artificiall - vedi - Esplodenti - Pirotecnia

Gallinacel - vedi Animali da cortile - Colombi - Pollicoltara.

Galvanizzazione, pulitura e verniciatura dei metalli e galvanoplastica in generale. Manuale pratico per l'industriale e l'operaio riguardante la nichelatura, ramatura, ottonatura, doratura, argentatura, stagnatura, zincatura, acciaiatura, antimoniatura, cobaltatura, ossidatura, galvinoplastica in rame, argento, oro, ecc., in tutte le varie applicaz. pratiche, di F. WERTH, ải p. XVI-324, con 153 inc. 50 Galvanoplastica ed altre applicazione dell'elettrolisi. Galvanostegia, Elettrometallurgia, Affinatura dei metalli, Preparazione dell'allaminio, Sbianchimento della carta e delle stoffe, Risanamento delle acque, Concia elettrica delle pelli, ecc., del Prof. R. FERRINI, $3^{a}$ edizione, completamente rifatta, pag. XII-417, con 45 incisioni . . . . . $4-$

Galvanostegia, dell' Ing. I. GHERsI. Nichelatura, argentatura, doratura, ramatura, metallizzazione, ecc. pag. XII324 , con 4 incisioni.

Gastronomia (Terminologia gastronomica italiana e francese) di E. Borgorello, con 300 Menus. (ln lavoro).

Gaz illuminarte (Industria del), di V. Calzavara, pag. XXXII-672, con 375 incisioni e 216 tabelle . . . . 750 - vedi Incandescenza a gaz.

Gelsicoltura, del Prof. D. TAMaro, pag. xVI-175 e 22 inc. 2 Geografia, di G. Grove, traduzione del Prof. G. GALLETTI, $2^{8}$ edizione riveduta, pag. XII-160, eon 26 incisioni . . 150 
Geografia classica, di H. F. TozER, traduzione e note del

Prof. I. GenTILE, 5a edizione, pag. IV-168

Geografia commerciale economica. Europa, Asia, Oceania, Africa, América, di P. LANZONI, $2^{2}$ edizione, di pag. VII-370

Geografia fisica, di A. GELKIE, traduzione di A. STOPPANI, $3^{2}$ edizione, pag. IV-132, con 20 incisioni . . . . . 150 Geologia, di A. GEIKIE, traduzione di A. STLPPANI, quarta edizione, rivednta sull'ultima edizione inglese da G. MERCALII, pag. $\pi \pi 176$, con 47 incisioni : . . . . . 150 Ceometria analitica dello spazio, del Prof. F. AschIERI, pag. VI-196, con 11 incisioni.

Ceometria analitica del piano, del Prof. F. AscHIERI, di pag. VI-194, con 12 incisioni

Geometria descrittiva, del Prof. AscHIERI, pag. VI-222, con 103 incisioni, $2^{\text {a }}$ edizione rifatta. . . . . . . 150 Geometria oiementare - vedi Esercizi di Geometria pura - Complementi di Geometria - Probiemi di Geometria elementare.

Geometria o trigonometria della sfera, del Prof. C. ALAรI.A, pag. VIII-208, con 34 incisioni . . . . . . . 150 Seometria metrica e trigonometria, del Prof. S. PINCEERLE, 6a edizione, pag. IV-158, con 47 incisioni. . . 150 - vedi anche Isercizi di Trigonometria.

Beometria pratica, dell'Ing. Prof. G. EREDE, $3^{\text {a }}$ edizione riveduta ed sumentata, pag. XIr-258, con 134 incis. . . 2 Geometria projettiva del piano e della stella, del Prof. F. AschIERI, 2a edizione, pag. VI-228, con 86 incisioni. . 150 Geometria projettiva ciello spazio, del Prof. F. AschIERI, $2^{2}$ edizione rifatta, pag: VI-264, con 16 incisioni . . 150 Geometria pura elementare, del Prof. S. PINCHERLE, $6^{a}$ edizione, con l'aggiunta delle figure sferiche, pag. vIII176, con 121 incisioni. . . . . . . . . . . 150 Ghiaccio - vedi Industria frigorifera.

Giardino (II) infantile, di P. CoNTI, pag. IV-213, 27 tav. 3 Ginnastica (Storia delia), di F. VALreTtr, pag. VIrr-181 . 150 Ginnastica femminile, di F. VALLETTX, pag. VI-112, 67 ill. 2 Gianastica maschile (Manuale di), per cura di J. GELLI, pag. VIII-108, con 216 incisioni

- vedi anche Giuochi ginnastici.

Gioielleria, oreficeria, oro, argento e platino, di E. BoSELII, pag. 336, con 125 incisioni.

- vedi anche Metalli preziosi - Pietre preziose. 
Giuochi ginnastici per la gioventù delle Scuole e del popolo, di F. GABRIELLI, pag. XX-218, con 24 tavole . . 250 Giuoco (Il) del pallone e gli altri affini. Giuoco del calcio (Foot-Ball), della palla a corda (Lawn-Tennis), della palla al muro (Pelota), della palla a maglio e dello sfratto, di G. Franceschi, di pag. VIII-214, con 34 incisioni . . 250 Giurato, (Manuale per il) del D.r A. SETTI. $2^{a}$ edizione rifatta, di pag. XIV-246.

urisdizione volontaria (Manuale della) e degli affari da trattarsi in eamera di consiglio, del Dott. A. Formentano (In lavoro).

Giustizia amministrativa. Principî fondamentali, Competenze dei Tribunali ordinari, Competenza della IV Sezione del Consiglio di Stato e delle Giunte provinciali amministrative e relativa procedura, di C. VITTA, p. XII-427 . Glottologia, del Prof. G. DE GREGorIo, pag. XXXII-318 . 3 Gnomonica ossia l'arte di costruire orologi solari, lezioni popolari di B. M. LA LETA, pag. VIII-160, con 19 figure. 2 -Eomma oiastica - vedi Imitazioni.

Grafologia, di C. Lombroso, pag. $\nabla-245$ e 470 fac-simili. 3 Grammatica albanese con le poesie rare di Variboda, del Prof. V. LibRandi, pag. XVI-200

Arammatica Araba - vedi Arabo parlato.

Grammatica araldica - vedi Araldica - Vocabolario araldico.

Grammatica ed esercizi pratici della lingua danesenorvegiana con un supplemento contenente le principali espressioni tecnico-nautiche ad uso degli nfficiali di marina che frequentano i mari del nord e gli stretti del Baltico, di G. FRISONI, pag. XX -488 .

Erammatica ed esercizi pratici della lingua ebraica, del Prof. I. Levi fu ISACCo, pag. 192 . . . . . . 150 Grammatica francese, del Prof. G. PRAT, seconda edizione riveduta, pag. XII-299 . . . . . . . . . 150

Grammatica o dizionario della lingua dei Galla coromonica), del Prof. E. Viterbo:

Vol. 1. Galla-Italiano, pag. VIII-152 . . . . . . 250

Vol. II. Italiano-Galla, pag. LXIV-106 . . . . . 250 Brammatica gotica - vedi Lingua gotica.

Grammatica greca. (Nozioni elementari di lingua greca), del Prof. InAMA. $2^{\mathrm{a}}$ edizione, pag. XVI-208 . . . . 150 Grammatica della lingua greca moderna, del Prof. $R$. LOVERA, pag. VI-154 . . . . . . . . . . . . . 150 Grammatica inglese, del Prof. L. PAviA, pag. XII-260 - 150 Grammatica italiana, del Prof. T. CONCARI, $2^{\mathrm{a}}$ edizione riveduta, pag. XVI-230.

Grammatica latina, L. VALivagGI, 2a ediz, pag. viII-256 150 Grammatica della lingua olandese, M.MORGANA, p.VIII-124 3 - 
Grammatica ed esercizi pratici della lingua portoghesebrasiliana, del Prof. G. FRISONI, pag. XII-267.

Grammatica e vocabolario della lingua rumena, del Prof. R. LOVERA, pag. VIII-200.

Grammatica russa, del Prof. VorNovich, pag. x-272 . 3 Grammatica sanscrita - vedi Sanscrito.

Grammatica della lingua slovena. Esercizi e vocabolario del Prof. BRUNo GUYON, pag. XVI-314.

Grammatica spagnuola, del Prof. PAVIA, di pag. IX-194 150 Grammatica della lingua svedese, del Prof. E. PÀroLI, pagine $X \nabla-293$.

Grammatica tedesca, del Prof. PAvIA, 2a ed., di p. XVIII-272 150 Grarnmatica Tigré - vedi Tigrè italiano.

Grammatica turca osmanli, con paradigmi, erestomazia, e glossario, di L. BoNELI, pag. VIII-200 e 5 tavole . . s Grandine - vedi Assicurazioni.

Granturco - vedi Frumento e mais - Industria dei molini.

Gravitazione. Spiegazione elementare delle principali perturbazioni nel sistema solare, di Sir G. B. AIRY, traduzione di F. Porro, con 50 incisioni, pag. XXII-176 . . 150 Grecia antica - vedi Archeologia (Arte greca) - Mitologia greca - Monete greche - Storia antica.

Gruppi continui di trasformazioni (Parte generale della teoria), di E. PASCAL, di pag. XI-378

Guida numismatica universale, contenente 6278 indirizzi e cenni storico-statistici di collezioni pubbliche e private, di numismatici, di società e riviste numismatiche, di incisioni, di monete e medaglie e di negozianti di monete e libri di numismatica, di F. GNECCHI. Quarta ediz., di pag. XV-612 8 Guttaperca - vedi Imitazioni.

Humus $\left(L^{\prime}\right)$, la fertilità e l'igiene dei terreni culturali, del Prof. A. CASALI, pag. XVI-210 . . . . . . . . $2-$ Idraulica, T. PERDONI, pag. XXVIII-392 con 301 fig. e 3 tav. 650 Idrografia - vedi Fotogrammetria

Idroterapia, di G. GIBELLI, pag. IV-238, con 30 incis. . . 2 - vedis anche Acque minerali e termali del Regno d'Italia.

Igiene della Bocca e dei Denti, nozioni elementarj di 0 iontologia, di L. CoULLIAUX, di pag. XVI-330, e 23 inc. 250 Igiene del lavoro, di TrambUSTI A. e SANARELLI, pagine VIII-262, con 70 incisioni. . . . . . . . . 250 Igiene della pelle, di A. BeLLINI, pag. XVI-240, 7 incis. . 2 Igiene privata e medicina popolare ad uso delle famiglie, di C. Bock, $2^{\text {a }}$ edizione italiana curata dal Dott. GIOv. GALLI, pag. XVI-272

Giene . . 250

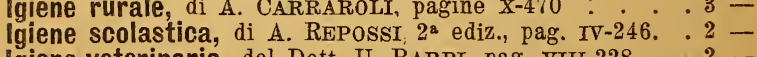
Igiene veterinaria, del Dott. U. BARPI, pag. VIU-228 . . 2 Igiene della vista sotto il rispetto scolastico, del Dott. A. LOMONAC0, pag. XII-272. 
Igiene della vita pubblica eprivata, G. FARALLI, p. XII-250 250 lgrescopi, igrometri, umidità atmosferica, del Prof. P. CANTONI, pag. XII-142, con 24 incisioni e 7 tabelle . . 150 illuminazione - vedi Acetilene - Gaz. illnm. - Incandescenza. Illuminazione elettrica (Impianti di), Manuale pratico dell'Ing. E. PIAZzOLI, 5 ${ }^{2}$ ediz. interamente rifatta, $(9-10 \mathrm{mi}-$ gllaio) seguita da un'appendice contenente la legislazione Italiana relativa agli impianti elettrici, di pag. 606 , con 264 incisioni, 90 tabelle e 2 tavole . . . . . . 650 Imbalsamatore - vedi Naturalisia preparatore - Naturalista viaggiatore - Zoologia.

imenotteri, Neurotteri, Pseudoneurotteri, Ortotteri e Rincoti italiani, del Dott. E. GRIFFINI (Entomologia IV), pag. XVI-687, con 243 incisioni

Imitazione di Cristo (Della), Libri quattro di Gro. GERSENIO, volgarizzamento di CESARE GUASTI, con proemio e note di G. M. ZaMPINI, pag. LVI-396

Imitazioni e succedanei nei grandi e piccoli prodotti industriali. Pietre e materiali da costruzione, Materiali refrattarii, Carborundum, Amianto, Pietre e metalli preziosi, Galvanoplastica. Cuoio, Linoleum, Seta e fibre tessili diverse, Paste da carta, Materie plastiche, Colle e gomme, Gomma elastica e Guttaperca, Avorio, Corno, Ambra, Madreperla, Celluloide, Viscoso, Cere e grassi, Materie concianti, Legno, Agglomerati di carbone, di segatura, di sughero, Polvere pirica, Caffè, Profumi, Vetrerie, ecc., dell'Ing. I. GHERSI, di pag. XVI-591, con 90 incisioni . 650 lamunità e resistenza alle malattie, di A. GALLI VALERIO, pag. VII-218.

Impiego ipodermico e la dosatura dei rimedi, Manuale di terapeutica del Dott. G. MALACRIDA, pag. 305 . . . Imposte dirette (Riscoss. delle), E. BRUNI, pag. VIII-158. 150 Incardescenza a gaz, (Fabbricazione delle reticelle) di $\mathrm{L}$. CASTELLANI, pag. $X-140$, con 33 incisioni. Inchiostri - vedi Ricettario industriale - Vernici ecc. Incisioni - vedi Amatore d'oggetti d'arte e di curiosità. Indovinelli - vedi Enimmistica.

Industria (L') frigorifera di P. ULIvI. Nozioni fondamentali, macchine frigorifere, raffreddamento dell'aria, ghiaccio artificiale e naturale, dati e calcoli numerici, nozioni di fisica e cenni sulla liquefazione dell'aria e dei gaz, di pagine XII-168, con 36 figure e 16 tabelle industrie olettrochimiche - vedi Distillazione del legno.

Industrie (Piccole). Scuole e musei industriali - Industrie agricole e rurali - Industrie manifatturiere ed artistiche, di I. GHERsI, $2^{\text {a }}$ ed. completam. rifatta del Mannale delle Piccole Industrie del Prof. A. ERRERA. pag. XII-372 . 350 Infanzia - vedi Terapia delle malattie dell' - Giardino infantile - Nutrizione - Ortofrenia - Sordomuto.

Infezione - vedi Disinfezione - Medicatura antisettica. 
Inioriunii sui lavoro - vedi Legge sugli.

L. c.

Infortunii della montagna (Gli). Manuale pratico degli Alpinisti, delle gride e dei portatori, del Dott. O. BERNHARD, traluzione con aggiunte del Dott. R. CurTI, di pag. XVIII-60, con 65 tav. e 175 figure dimostrative. . 350 Infortuni sul lavoro, (Mezzi tecnici per prevenirli) di E. NAGRINI, di pag. XXXII-252, con 257 incisioni. . . 3 - vedi anche Leggi per gli.

Ingegnere agronomo - vedi Agronomia - Prontuario dell'agric. Ingegnere civile. Manuale dell'ingegnere civile e industriale, del Prof. G. COLOMBO, 20 edizione modificata e aumentata, (520 al $54^{\circ}$ migliaio), con 221 fig., pag. XIV-423 . . 550

Il medesimo tradotto in francese da P. MARCILLAC .550 Ingegnere navale. Prontuario di A. CIGNONI, pag. XXXI292 , con 36 figure. Legato in pelle . . . . . . . 550 Ingegnere rurale (Prontuario dell') - Vedi Agricoltore.

Ingegneria legale per tecnici e giuristi (Manuale di), dell'Avv. A. LION. Commento ed illastrazione con la più recente giurisprudenza: Responsabilità - Perizia - Servitù Piani regolatori e di ampliamento - Legge di sanitá - Regolamenti d'igiene ed edilizii - Espropriazione - Miniere Foreste - Catasto - Privativa industriale - Acque - Strade Ferrovie - Tramvay - Bonifiche - Telefoni - Appalti - Riparazioni - Cimiteri - Derivazioni di acque pubbliche - Monnmenti d'arte e d'antichità, ecc., pag. VHI-552 . . . . . 550 Inghilterra - vedi Storia d'Inghilerra.

Insegnamento (L") dell'Italiano nelle Scuole Secondarie. Esposizione teorico-pratica con esempi, del Prof. C. TRABALZA, di pag. XVI-254

Insetti nocivi, del Prof. F. FRANCEschinI, pag. VIII-264, con 96 incisioni

Insetti utili, del Prof. F. Franceschini, di pag. xil-160, con 43 incisioni e 1 tavola

Interesse e sconto, del Prof. E. GaGliard, $2^{\text {a }}$ edizione rifatta e aumentata, pagine VHI-198.

Inumazioni - vedi Morte vera.

Ipnotisno - vedi Magnetismo - Spiritismo - Telepatia.

Ipoteche (Man. per le), di A. RABBENo, pag. XVI-247

Islamismo (L') del Prof. I. PIZZI, di pag. VIII-494.

Ittiologia italiana, del Dott. A. GRIFFinI, con 244 incis. Descriz. dei pesci di mare e d'acqua dolce, di p. XVIII-469 450 - vedi anche Piscicoltura - Ostricoltura.

Lacche - vedi Vernici ecc.

Latte, burro e cacio. Chimica analitica applicata al caseificio. del Prof. SARTORI, pag. X-162, con 24 incisioni . 2 Laveri femminili - vedi Abiti per signora - Biancheria - Macchine da cncire - Monogrammi - Trine a fuselli.

Lavori marittimi ed impianti portuali, di F. BAstranr, di pag. XXII-424, con 209 figure. . . . . . .6 50 
Lavori in terra (Manuale di), dell'Ing. B. LEONI, pag. XI305 , con 38 incisioni

Lavoro (Ii) delle donne e dei fanciulli. Nuova legge e regolamento 19 giugno $1902-28$ febbraio 1903 . Testo atti parlamentari e commento, per cura dell'Avv. E. NoSEDA di pag. XV-174 . . . . . . . . . . . . . 150

Lawn-Tennis, di V. BADDELEY, prima traduzione italiana con note e aggiunte del trad., pag. XXX-206, con 13 illustr. 250 Legge (La nuova) comunale e provinciale, annotata da $\mathrm{E}$. MAzZOCCOLO, $4^{\text {a }}$ edizione, interamente rifatta con l'aggiunta del regolamento e di 2 indici, pag. XII-820 . . 750

Legge sui lavori pubblici e regolamenti, di L. FRANCHI, pag. IV-110-CXLVIII .

Logge lavoro donne e fanciulli - vedi lavoro.

Legge. sull'ordinamento giudiziario, dell'Avv. L. FRANCH, pag. IV-92-CXXVI. . . . . . . . . . . . 150 Leggende popolari di E. MUSATTI. 3 ediz. di p. VIII-181 150 Leggi e convenzioni sui diritti d'autore - vedi Codici e leggi usuali d' Italia, vol. III.

Leggi per gl'Infortunil sul lavoro, dell'Avv. A. SalvaTORE, pag. 312

Leggi e convenzioni sulle privative industriali, disegni, modelli di fabbrica, marchi di fabbrica e di commercio, di L. FRANCHI. (In lavoro).

Leggi sulla sanità e sicurezza pubblica, di L. FRANCHI, pag. IV-108-XCII . . . . . . . . . . . . 150

Leggi sulle tasse di Registro e Bollo, con appendice, del Prof. I. FRANCHI, pag. IV-124-CII . . . . . . . . 150 Leggi usuali d'Italia - vedi Codici e leggi.

Leghe metalliche ed amalgame, alluminio, nichelio, metalli preziosi e imitazione, bronzo, ottone, monete e medaglie, saldature, dell'Ing. I. GHERSI, p. XVI-431, con 15 inc. 4 -

Legislazione delle acque di D. CAVALLERI, di pag. xv-274 250 Legislazione Mortuaria - vedi Morte.

Legislazione sanitaria italiana, (La nuova) di E. NOSEDA. di pag. VIII-570.

Legislazione rurale, secondo il programma governativo per gli Istituti Tecnici, dell'Avv. E. BRUNI, pag. XI-423 . . 3Legnami - vedi Cubatura dei legnami - Falegname.

Legno artificiale - vedi Imitazioni.

Legno (Lavorazione dei prodotti di distillazione del) - vedi Distillazione.

Lepidotteri italiani, del Dott. A. GRIFFINI (Entomol. II), pag. XIII-248. con 149 incisioni. . . . . . . . . 150

Letteratura albanese (Manuale di), del Prof. A. STRATicò, pag. XXIV-280.

Letteratura americana, di G. STRAFFoRELLo. pag. 158150

Letteratura araba, del Prof. I. PrzzI di pag. xII-388 . 3 - vedi anche Islamismo. 
Letteratura assira, del Dott. B. TELONI di pag. xv-266 e

L.c. tre tavole fuori testo

Letteratura catalana, del Prof. RESTORI. (In lavoro).

Letteratura danese - vedi Letteratura norvegiana

Letteratura drammatica, di C. LEVI, pag. XIr-339. . . 3 -

Letteratura ebraica, di A. REvEL, 2 vol., pag. 364 . . 3 -

Letteratura egiziana, di L. BRIGIUTr. (In lavoro).

Letteratura francese, del Prof. E. Marcillac, traduz. di A. PAGANINI, 3a edizione, pag. VIII-198 . . . . . 150

Letteratura greca, di V. INAMA, $14^{\mathrm{a}}$ ediz. riveduta (dal $56^{\circ}$ al $61^{\circ}$ migliaio) pag. VIII-236 e nna tavola. . . 150

Letteratura indiana. A. DE GUBERNATIS, pag. VIII-159 . 150 Letteratura inglese, di E. SolAzzI, $2^{\text {a }}$ edizione, di pagine VIII-194. . . . . . . . . . . . . . 150

Letteratura italiana, del Prof. C. FENINI, dalle origini al $1748,5^{a}$ edizione completamente rifatta dal Prof. V. FERRARI, pag. XVI-291 . . . . . . . . . . 150 Letteratura italiana moderna, (1748-1870). Aggiunti 2 quadri sinottici della letteratura contemporanea (1870-1901) del Prof. V. FERRARI, pag. 290 . . . . . . 150

Letteratura italiana moderna e contemporanea 17481901, del Prof. V. FERRARI, pag. VIII-406. . . . . 3 Letteratura latina - vedi Letteratura romana

Letteratura norvegiana, di S. CoNsoLI, pag. XVI-272. . 150 Letteratura persiana, del Prof. I. PJZZI, pag. X-208 . . 150 Letteratura provenzale, di A. RESTORI, pag. X-220 . . 150 Letteratura romana, del Prof. F. RAMORINO, 6ª edizione corretta, di pag. VIII-349. . . . . . . . . 150

Letteratura spagnuola e portoghese, del Prof. L. CAPPELLETTI, $2^{2}$ edizione rif. da B. SANVISENTI (In lavoro). Letteratura tedesca, del Prof. O. LANGE, $3^{2}$ edizione rifatta dal Prof. MINUTTI, pag. XVI-188 . . . . . 150

Letterafura ungherese, di ZIGANY ARPÀD, pag. XII-295. 150 Letterature slave, del Prof. D. CIÀMPOLI, 2 volumi:

I. Bulgari, Serrbo-Croati, Yngo-Russi, pag. IV-144. . . 150

II. Russi, Polacchi, Boemi, pag. IV-142 . . . . . 150

Lexicon Abbreviaturarum quae in lapidibas, codicibus et chartis praesertim Medii-Aevi occurrunt - vedi Dizionario di abbreviature.

Limoni vedi Agrumi.

Lingua araba - vedi Arabo parlato - Dizionario eritreo - Grammatica Galla - Lingue dell'Africa - Tigrè.

Lingua gotica, grammatica, esercizi, testi, vocabolario comparato con ispecial riguardo al tedesco, inglese, latino $\theta$ greco, del Prof. S. FRIEDMANN, pag. XVI-3333

Lingua greca - vedi Esercizi - Filologla - Florilegio - Grammatica -- Jetteratura - Morfologia - Dialetti - Verbi. 
Lingue dell'Africa, di R. CUST, versione italiana del Prof. A. De GUbernatis, di pag. IV-110 . . . . . 150 Lingua latina vedi Dizionario di abbreviature latine - Epigrafia - Esercizi - Filologia classica - Fonologia - Grammatica - - Letteratura romana - Metrica - Verbi.

Lingue germaniche - vedi Grammatica danese-norvegiana inglese, olandese, tedesca, svedese.

Lingua Turca Osmanli - vedi Grammatica.

Lingue neo-latine, del Dott. E. GorRA, pag. 147. . . . 150

Lingue straniere (Studio delle), di C. MARCEL, ossia l'arte di pensare in una lingua straniera, traduzione del Prof. DAMIANI, di pag. XVI-136 . . . . . . . . . 150 Linoleum - vedi Imitazioni.

Liquidatore di sinistri maritzimi - vedi Avarie e sinistri marittimi.

Liquorista, di A. RossI, con 1270 ricette pratiche. Nateriale, Materie prime, Manipolazioni, Tinture, Essenze uar turali ed artificiali, Fabbricazione dei liquori per macerazione, digestione, distillazione, con essenze, tinture, ecc., Liquori speciali, Vini aromatizzati, pag. XXXII-560, con 19 incisioni nel testo . . . . . . . . . . . 5 -

Litografia, di C. DOYEN, di pag. VIrI-261, con 8 tavole e 40 figure di attrezzi, ecc., occorrenti al litografo . . . 4 Liuto - vedi Chitarra - Mandolinista - Strum. ad arco.

Logaritmi (Tavole di), con 6 decimali, di O. MÜLLER, 6a ediz., aumentata delle tavole dei logaritmi d'addizione e sottrazione per cura di M. RAINA, di pagine XXXVI-191. $\left(11,12,13^{\circ}\right.$ migliaio $) . . . . . . .150$ Logica, di W. Stanley Jevons, traduz. del Prof. C. CanTONI, 5a ediz. di pag. VIII-166, con 15 incisioni. . . 150 Logica matematica, del Prof. C. BURALI-FORTI, p. VI-158. 150 Logismografia, di C. CHIESA, 3a ediz., pag. XIV-172 •. 150 Logogrifi - vedi Enimmistica.

Lotta - vedi Pugilato.

Luce e colori, del Prof. G. Bellotti, pag. $x-157$, con 24 incisioni e 1 tavola. . . . . . . . . 150

Luce e suono, di E. JoNES, traduzione di U. ForNARI, di pag. VIII-386, con 121 incisioni. . . . . . . . 3 -

Luce e salute. Fototerapia radioterapia del Dott. A. BELLINI, di pag. XII-362, con 65 figure . . . . 350

Macchine (Atlante di) e di Caldaie, con testo e note di Tecnologia, di pag. Xv-80, con 112 tavole e 170 figure in iscala ridotta

Macchine a vapore, (Manuale del costruttore di), di H. HAEDER. Edizione italiana compilata sulla $5^{a}$ edizione tedesea, con notevoli aggiunte dell'Ing. E. WEBBER, pag. XVI-452, con 1444 incisioni e 244 tabelle, legato in bulgaro rosso . 7 -

Macchine agricole. del Conte A. CENCELLI-PERTI, di pag.

VIII-216, con 68 incisioni . 
Macchine per cucire e ricamare, dell'Ing. ALFREDo GAL. c. LASSINI, pag. VII-230, con 100 incisioni

Macchinista e fuochista, del Prof. G. GAUTERo, riveduto e ampliato dall'Ing. Prof. LorIA, 9a ediz. con Appendice sulle Locomobili e le Locomotive e col Regolamento sulle caldaie a vapore, pag. $\mathrm{xx}-194$, con 34 incisioni. . . .

Macchinista navale (Manaale del), di M. LigNaroLo, $2^{a}$ ed. rifatta, pag. XXIV-602, con 344 incisioni .

Macinazione - vedi Industrie dei molini - Panificazione.

Magnetismo ed elettricità. Principí e applicazioni esposti elementarmente, del Prof. F. GRASSI. 3a ediz. completamente rifatta del manuale di POLONI e GRASSI, di pagine XVI-508, con 280 figure 6 tavole fuori testo

Magnetismo ed ipnotismo, del Dottor G. BELFIORE, $2^{\mathrm{a}} \mathrm{ed}$. rifatta pag. VIII-396.

Maiale (II). Razze, metodi di riproduzione, di allevamento, ingrassamento, commercio, salumeria, patologia suina e terapentica, tecnica operatoria, tossicologia, dizionario snino-tecnico, del Prof. E. MARCHI, $2^{\text {a }}$ ediz., pag. XX-736, con 190 incisioni e una Carta.

Misaliche e porcellane (L'amatore di), di L. DE MAURI, ilIustrato da splendide incisione in nero, da 12 superbe tavole a colori e da 3000 marche. - Contiene: Tecnica della fabbricazione - Sguardo generale sulla storia delle Ceramiche dai primi tempi fino ai giorni nostri - Cenni storici ed artistici su tutte le fabbriche - Raccolte di 3000 marche corredate ognuna di notizie relative, e coordinate ai Cenni Storici in modo che le ricelche riescano di esito immediato Dizionario di termini Artistici aventi relazione coll'Arte Ceramica e di oggetti Ceramici speciali, coi prezzi correnti. Bibliografia ceramica, indici vari, pag. XI-650 . . . . 1250

Mais (II) o granoturco, o formentone, o granone, o melgone, o melica, o melicotto, o carlone, o polenta, ecc. Norme per una buona coltivazione, di E. AzIMONTI, $2^{2}$ edizione rifatta dal Manuale "Frumento e Mais , di E. CANTONI, di pag. XII-196 con 61 incisioni nel testo .

Malattie dei paesi caldi, del Dott. C. Muzio. (In lavoro). Malattie crittogamiche delle piante erbacee coltivate, del Dott. $R$. WOLF, traduzione con note ed aggiunte del Dott. P. BACCARINI, pag. $X-2 i 8$, con 50 incisioni .

Malattie ed alterazione dei vini, del Prof. S. CETTOLnNI, di pag. XI-138, con 13 incisioni.

Malatie della vite - vedi Filossera - Malattie crittogamiche. Mammiferi - vedi Zoologia.

Mandarini - vedi Agrumi.

Halattie del sangue. Mannale d'Ematologia del Dott. E. REBUSCHNI, pag. VIII-432

Mandato sommerciale, di E. VIDARI, pag. VI-160. 
Mandolinista (Manuale del), di A. PISANI, pag. XX-140, con

13 figure, 3 tavole e 39 esempi.

Manicomio - vedi Assistenza pazzi - Psichiatria.

Manzoni Alessandro. Cenni biografici, di L. BeLTRAMI, di pag. 109 , con 9 autografi e 68 incisioni. . . . . . 150 Marche di Fabbrica - vedi Amatore oggetti d'arte - Leggi sulle proprietà - Majoliche.

Mare (II). V. BELLIO, pag. IV-140, con 6 tav. lit. a colori. 150 Marine (Le) da guerra del mondo al 1897, di.L. D'ADDA, pag. XVI-320, con 77 illustrazioni. . . . . . . 450 Marino (Manuale del) militare e mercantile, del Contr'ammiraglio DE AMEZAGA, con 18 xilografiie, 2a edizione, con appendice di BuCCI DI SANTAFIORA . . . . . 5 -

Marmista (Manuale del), di A. RICCI, $2^{\text {a }}$ edizione, pag. XII154, con 47 incisioni

Marmo - vedi Imitazioni.

Massaggio, del Dott. R. MajnonI, p. XII-179, con 51 inc. . 2 Mastici - vedi Ricettario industriale - Vernici, ecc.

Matematica (Complementi di) ad uso dei chimici e dei naturalisti, di G. VIVANTI, di pag. X-381 . . . . . 3Matematiche superiori (Repertorio di). Definizioni, formole, teoremi, cenni bibliografici, del Prof. E. PAscal.

Vol. I. Analisi, pag. XVI-642 . . . . . . . .6-

Vol. II. Geometria, e indice gen. per i 2 vol. pag. 950950 Materia medica moderna (Man. di), G. MALACRIDA, p. XI-761 750 Materiali artificiali - $v$. Ricettario indust. - Imitaz. $\theta$ succedanei. Meccanica, del Prof. R. STAWELl BALL, traduzione del Prof. J. BENETTI, $4^{\mathrm{a}}$ edizione, pag. XVI-214, con 89 inc.. 150 Meccanica (La) del macchinista di bordo, per gli Ufficiali macchinisti della R. Marina, i macchinisti delle Compagnie di navigazione, i Costruttori e i Periti meccanici, gli Allievi degli Istituti Tecnici e Nautici e delle Scuole Industriali e Professionali, di E. GIORLI, con 92 figure . . .

Meccanico (Il), ad uso dei capi tecnici, macchinisti, elettricisti, disegnatori, assistenti, capi operai, conduttori di caldaie a vapore, alunni di Scuole industriali, di E. GIORLI, $4^{\text {a }}$ edizione ampliata, pag. xVI-424, con 204 incisioni . . 3 Meccanismi $(500)$, scelti fra i più importanti e recenti riferentisi alla dinamica, idraulica, idrostatica, pneumatica, macchine a vapore, molini, torchi, orologerie, ecc., di $\mathrm{H}$. T. BRown, trad. d. Ing. F. CERRUTI, $3^{a}$ edizione italiana, pag. VI-176, con 500 incisioni . . . . . . . . 250 Modaglie - vedi Leghe metalliche - Monete greche - Monete romane - Numismatica - Vocabolarietto dei numismatici.

Âedicatura antisettica, del Dott. A. ZAMBLER, con prefazione del Prof. E. TRIToNI, pag. XVI-124, con 6 incis. . 150 
Medicina operativa - vedi Chirurgia.

Medico pratico, (II) di C. Muzro. $3^{a}$ edizione del Nuovo memoriale pei medici pratici, di pag. XVI-492 . . . . 5 Wemoria (L'arte della) - vedi Arte.

Mercedi - veải Paga giornaliera.

Merciologia, ad uso delle scuole e degli agenti di commercio, di O. LUXARDo, pag. XII-452.

Meridiane - vedi Gnomonica.

Metalli preziosi (oro, argento, platino, estrazione, fusione, assaggi, usi), di G. GoRINI, $2^{2}$ ed., p. II-196, con 9 inc. . 2 Metallizzazione - v. Galvanizz.-Galvanoplastica - Galvanostegia. Metallocromia. Colorazione e decorazione chimica ed elettrica dei metalli, bronzatura, ossidazione, preservazione e

pulitura, dell'Ing. I. GHERSI, pag. VIII-192. . . . . 250 Metallurgia - vedi Coltivazione delle miniere - Fonditore Leghe metalliche - Siderurgia - Tempera e cementazione.

Meteorologia generale, del Dott. L. DE MarchI, pag. VI156, con 8 tavole colorate. . . . . . . . . 150 vedi anche - Climatologia - Igroscopi.

Metrica dei greci e dei romani, di L. MÜLLER, $2^{\text {a }}$ edizione italiana confrontata colla $2^{\mathrm{a}}$ tedesca ed annotata dal Dott. GIUSEPPE ClERICO, pag. XVI-186.

Metrica italiana - vedi Ritmica e metrica italiana.

Metrologia Universale ed il codice Metrico Internazionale, coll'indice alfabetico di tutti i pesi misure, monete, ecc., dell'Ing. A. TACCHINI, pag. $x x-482$

Mezzeria (Manuale pratico della) e dei vari sistemi della colonia parziaria in Italia, d. Prof. A. RABEENO, p. VIII-196 150 Micologia vedi Funghi mangerecci - Malattie crittogamiche - Tar. tufi e funghi.

Microbiologia. Perchè e come dobbiamo difenderci dai microbi. Malattie infettive, Disinfezioni, Profilassi, del Dott.

L. PIZZINI, pag. VIII-142 .

woroscopia - vedi Anatomia microscopica - Animali parassiti Bacologia - Batteriologia - Prostitologia - Tecnica prostitologica.

Wicroscopio (II), Guida elementare alle osservazioni di Microscopia, del Prof. CAMILLO ACQUA, p. XII-226, 81 inc. 150 Mineralogia generale, del Prof. L. BOMBICCI, $2^{a}$ ediz. liveduta, di pag. XVI-190, con 183 inc. e 3 tavole. . . 150 翟ineralogia descrittiva, del Prof. L. BoMBICCI, $2^{\text {a }}$ edizione, di pag. IV-300, con 119 incisioni.

miniere (Coltivazione delle), di S. BERTolio, $2^{\text {a }}$ ediz. rifatta del Man. "Arte Min. " di ZOPPETṬ, p. VIU-284 . 250 Miniero di zolfo - vedi Zolfo. thisurazione delle botti - vedi Enologia. 
Misure - vedi Avarie e sinistri marittimi - Codice del Perito Misuratore - Metrologia - Monete - Strumenti metrici.

Mitilicoltura - vedi Ostricoltura - Piscicoltura.

Mitologia (Dizionario di), di F. RAMORINo. (In lavoro).

Mitologia comparata, del Prof. A. DE GUBERnatis, 2 edizione, di pag. VIII-150. (Esaurito).

Mitologia greca, di A. Poresti:

Volume I. Divinità, di pag. VIII-264 . . . . . 150

Volume II. Eroi, di pag. 188 . . . . . . . 150 A itologie orientali, di D. BASSI:

Vol. I. Mitologia babilonese-assira, pag. XVI-219 . 150

Vol. II. Mitologia egiziana e fenicia. (In lavoro).

Hinemotecnia - vedi Arte della memoria.

Mobili artistici - vedi Amatore d'oggetti d'arte.

Moda - vedi Abiti - Biancheria - Fiori artificiali - Trine.

Modellatore meccanion, falegname ed ebanista, del Prof.

G. MINA, pag. XVII-428, con 293 incisioni e 1 tavola . 550 Molini ( $L$ 'Industria dei) e la macinazione del frumento, di C. SIBER-MILLOT, di pag. XX-259, con 103 incisioni nel testo $\theta 3$ tavole.

Momenti resistenti e pesi di travi metalliche composte. Prontuario ad uso degli Ingegneri, Architetti e costruttori, con 10 figure ed una tabella per la chiodatura, dell'Ing. E. SCHENCK, di pag. XI-188. : . . . . . . . 350 Monete greche, di S. AMBRosoli, di pag. XIV-286, con 200 fotoincisioni e 2 carte geografiche.

Monete (Prontuario delle), pesi e misure inglesí, ragguagliate a quelle del sistema decimale, dell'Ing. GHERSI, di pag. XI-196, con 47 tabelle di conti fatti e 40 facsimili delle monete in corso . . . . . . . . . . 350

Monete romane. Manuale elementare compil. da F. GNECCHI, $2^{\text {a }}$ edizione, riveduta corretta ed ampliata, di pag. XXVII-370, con 25 tavole e 90 figare nel testo . . . . 3 Monogrammi, del Prof. A. SEvERI, 73 tavole divise in tre serie, le prime due di 462 in due cifre e la terza in 116 in tre eifre.

Montatore (II) di macchine. Opera arricchita da oltre 250 esempi pratici e problemi risolti, di S. DINARo, di pagine $\mathrm{XII}{ }_{y} 68$

Morfologia generale - vedi Embriologia.

Morfologia greca, del Prof. V. BetteI, pag. XX-376. . . 3 Morfologia italiana, del Prof. E. GoRra, pag. VI-142 . . 150 Morte (La) vera e la morte apparente, con appendice " La 


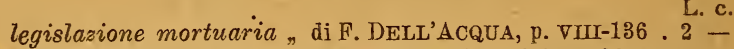
Mosti (Densità dei), dei vini e degli spiriti ed i problemi che ne dipendono, ad uso degli enochimici, degli enoteenici e dei distillatori, di E. CrLLIS, di pag. XVI-230, con 11 figure e 46 tavole.

Musei - vedi Amatore oggetti d'arte e curiosità - Amatore maioliche e porcellane - Armi antiche - Pittura - Scoltura.

Hutuo soccorso - vedi Societ⿱亠े mutuo soccorso

Napoleone $1 \circ$, di L. CAPPELLETTI, 23 fotuine., p. $\mathrm{xX}-272$. 250 Naturalista preparatore (II), del Dott. R. GESTRO, $3^{\mathrm{a}}$ edizione riveduta ed aumentata del Manuale dell'Imbalsamatore, di pag. XVI-168, con 42 incisioni. . . . . . 2Naturalista viaggiatore, del Prof. A. ISSEL e R. GESTRO (Zoologia), di pag. VIII-144, con 38 incisioni . . . . . 2 Nautica stimata o Navigazione piana di F. TAMI, di pag. XXXII-179, con 47 incisioni .

Meurotteri - vedi Imenotteri.

Nevrastenia del Dott. L. CAPPELLETTI di pag, xx-490 . 4 vichelatura - vedi Galvanostegia.

Notaio (Manuale del), aggiunte le Tasse di registro, di bollo ed ipotecarie, norme e moduli pel Debito pubblico, di A. GARETTI, 4a ediz. riveduta $\theta$ ampliata, pag. VIII-380. . 350 Numeri - vedi Teoria dei numeri.

Numismatica, del Dott. S. Ambrosoli, 3.a ediz. riveduta, di pag. XVI-250, con 250 fotoinc. e 4 tav. . . . . . 150 Numismatica - vedi Guida numismatica.

Nuotatore (Mannale del), del Prof. P. ABBo, di pag. XII148, con 97 incisioni . . . . . . . . . 250 Nutrizione del bambino. Allattam. naturale ed artificiale, del Dott. L. CoLombo, pag. XX-228, con 12 incisioni. . 250 Occultismo - vedi Magnet. e ipnotismo - Spiritismo - Telepatia. Oculistica - vedi Igiene della vista - Ottica.

Odontologia - vedi Igiene della bocca.

Olii vegetali, animali e minerali, loro applicazioni di G. GoRINI, $2^{\text {a }}$ edizione eompletamente rifatta dal Dott. G. FABRIS, di pag. VIII-214, con 7 incisioni . . . . . 2 Olivo ed olio. Coltivazione dell'olivo, estrazione, purificazione e conservazione dell'olio, del Prof. A. ALOI, $5^{2}$ edizione accresciuta e rinnovata, di p. XVI-365, con 65 inc. 3 Omero, di W. Gladostone, traduzione di R. Palumbo e C. FIORILLI, di pag. XII-196 Onde Hertziane - vedi Telegrafo senza fili.

Operaio (Manuale dell'). Raccolta di cognizioni utili ed indispensabili agli operai tornitori, fabbri, calderai, fonditori di metalli, bronzisti, agginstatori e meccanici di G. BELLUOMTNI, $5^{2}$ edi\%, aumentata, di pag. XVI-262 . 
Operazioni doganali - vedi Codice doganale - Trasporti e tariffe. Oratoria - vedi Arte del dire - Rettorica - Stilistica.

Ordinamento degli Stati liberi d'Europa, del Dott. F. RACIOPPI, $2^{a}$ edizione, di pag. XII-316 . . . . . . . 3 Drdinamento degli Stati liberi fuori d'Europa, del Dott.

F. RACIOPPI, di pag. VHI-376

Ordinamento giudiziario - vedi Leggi sull'.

Oreficieria - vedi Gioielleria - Leghe metalliche - Metalli preziosi - Saggiatore.

Organoterapia, di E. ReBUschINI, pag. $\nabla$ III-432 . . . . 3 50 Oriente antico - vedi Storia antica.

Ornatista (Manuale dell'), dell'Arch. A. MELANI. Raccolta di iniziali miniate e incise, d'inquadrature di pagina, di fregi e finalini, esistenti in opere antiche di biblioteche, musei e collezioni private. XXIV tavole in colori per miniatori, calligrafi, pittori di insegne, ricamatori, incisori, disegnatori di caratteri, ecc.. Ia serie, in-8 . . . . 450

Orologeria moderna, dell'Ing. GARUFFA, di pag. viII-302, con 276 incisioni.

- vedi anche Gnoilonica.

Orologi artistici - vedi Amatore di oggetti d'arte.

Orologi solari - vedi Gnomonica.

Orticoltura del Prof. D. TAMaro, $2^{a}$ edizione rifatta, di pagine XVI-576, con 110 incisioni . . . . . . . 450 Ortocrematismo - vedi Fotografia.

Ortofrenia (Mannale di), per l'educazione dei fanciulli frenastenici o deficienti (idioti, imbecilli, tardivi, ece.), del Prof. P. PARISE, di pag. XII-231.

Optotteri - vedi Imenotteri ecc.

Ossidazione - vedi Metallocromia.

Ostetricia (Manuale di) Ginecologia minore, per le levatrici di L. M. Bossr, di pag. XV-493, con 113 incisioni 450 Ostricoltura e mitilicoltura, del Dott. D. CARAzZI, con 13 fototipie, di pag. $\nabla \amalg 1-202$. . . . . . . . 250 Ottica, di E. GELCICH, pag. XVI-576, con 216 incis. e 1 tav. 6 Ottone - vedi Leghe metalliche.

Paga giornaliera (Prontuario della), da cinquanta centesimi a lire cinque, di C. NEGRIN, di pag. 22\%. . . . 250 Paleoetnologia, del Prof. J. REgAzzonI, di pag. XI-252, con 10 incisioni............. . . 150

Paleografia, di E. M. THompson, tradnzione dall'inglese, con agginnte e note del Prof. G. FUMAGaLLI, $2^{2}$ edizione rifatta, di pag. XII-178, con 30 inc. e 6 tav,. . . . . 2 -

Paleontologia (Compendio di), del Prof. P. VINassa DE REGNY, di pag. XVI-512, con 356 figure intercalate. . 550 
Pallone (Giuoco del) - vedi Giuoco.

Panificazione razionale, di PompILIo, pag. IV-126.

Parafulmini - vedi Elettricità - Fulmini.

Pazzia - vedi Psichiatra - Grafologia.

Pediatria - vedi Nutrizione del bambino - Ortopedia - Terapia malattie infanzia.

Pellagra (La), Storia, eziologia, patogenesi, profilassi. di $\mathrm{G}$. ANTONLNI, di pag. VIII-166 con 2 tav.

Peile - vedi Igiene della.

Pelli - vedi Concia delle pelli.

Pensioni - vedi Società di mutuo soccorso.

Pepe -- Prodotti agricoli.

Perfosfati - vedi Fosfati - Concimi - Chimica agraria.

Perito - vedi Codice nel perito misuratore - Ingegneria legale.

Pesci - vedi Ittiologia - Ostricoitura - Piscicoltura.

Pesi \& misure - vedi Avarie e sinistri marittimi - Metrologia Misure e pesi inglesi - Monete - Strumenti metrici - Tecnologia monetaria.

Peso dei metalli, ferri quadrati, rettangolari, cilindrici, a squadra, a U, a Y, a Z, a $T$ e a doppio T, e delle lamiere e tubi di tutti i metalli, di G. BELLUOMINI, di pag. XXIV-248.

Pianeti - vedi Astron. - Cosmogr. - Gravit. - Spettroscopio.

Pianista (Manuale del), di L. MastrIGLI, pag. XVI-112 . 2 Piante $e$ fiori sulle finestre, sulle terrazze e nei cortili. Coltnra e descrizione delle principali specie di varietà, di A. PUCCI, 2a edizione, pag. VIII-214, con 117 incisioni . 250 Piante industriali, coltivazione, raccolta, preparazione, di G. GORINI, nuova edizione, di pag. II-144.

Piante tessili (Coltivazione ed industrie delle), propriamente dette e di quelle che danno materia per legacci, lavori d'intreccio, sparteria, spazzole, scope, carta, ecc., coll'aggiunta di un dizionario delle piante ed industrie tessili, di oltre 3000 voci, del Prof. M. A. SavorgnaN D'OsopPo, di pag. $\mathrm{XII},-476$, con 72 incisioni.

Piccole industrie - vedi Industrie.

Pietre artificia!i - vedi Imitazioni.

Pietre preziose, classificazione, valore, arte del giojelliere, di G. TORINI, $2^{\text {a }}$ edizione, di pag. 138, con 12 incisioni. 2 Pirotecnia moderna, di F. DI MAIo, $2^{\mathbf{a}}$ edizione riveduta ed ampliata, di pag. $\mathrm{XV}-183$ con 21 incisioni. . . . 250

Piscicoitura (d'acqua dolce), del Dott. E. BETTONI, di pagine VIII-318, con 85 incisioni.

Pittura ad olic, acquarelio e miniatura (Manuale per dilettante di), paesaggio, figura e fiori, di G. RONCHETTI, pag. XVI-230, 29 incis. e 24 Tav. in zincot. e cromolit. . 350

Pittura italiana antica e moderna, dell'Arch. A. MELANI, 
$2^{\text {s }}$ edizione completamente rifatta, di pag. $\mathrm{XxX}-430$ con

23 incisioni intercalate e 137 tavole. . . . . . 750 Plastica - vedi Imitazioni.

Pollicoltura, del March. G. Trevisani, 5a edizione rifatta, di pagine XVI-230, con 90 incisioni . . . . . . 250 Polvori piriche - vedi Esplodenti - Pirotecnia.

Pomologia, descrizione delle migliori varietà di Albicocchi, Ciliegi, Meli, Peri, Peschi, del Dott. G. MoLon, con 86 incisioni e 12 tavole colorate, di pag. XXXI-717 . . 850 Pomologia artificiale, secondo il sistema Garnier-Valletti, del Prof. M. DEL LuPo, pag. VI-132, e 34 incísioni . . 2 Poponi - vedi Frutta minori.

Porcellane - vedi Maioliche - Ricettario domestico.

Porco (Allevamento del) - vedi Maiale.

Porti di mare - vedi Lavori marittimi.

Posologia - vedi Impiego ipodermico.

Posta. Manuale Postale di A. PALombi. Notizie storiche sulle Poste d'Italia, organizzazione, legislazione, posta militare, nnione postale universale, con nna appendice contenente le norme relative ad alcuni servizi accessori della posta, di pag. $\mathrm{xxx}-309$. . . . . . . . . . 3 -

Prato (II), del Prof. G. CANToNi, di pag. 146, con 13 inc. 2 Prealpi bergamasche (Guida-itinerario alle), compresa la Valsassina ed i Passi alla Valtellina ed alla Valcamonica, colla prefazione di A. STOPPANI, e cenni geologici di A. TARAMELLI, $3^{a}$ edizione rifatta per cura della Sezione di Bergamo del C. A. I., con 15 tavole, due carte topografiche, ed una carta e profilo geologico. Un volume di pag. 290 e un vol. colle carte topografiche in busta . .650 Pregiudizi - vedi Errori e pregiudizi. Leggende popolari.

Previdenza - vedi Assicuraz. - Cooperaz. - Societi di M. S. Privative industriali - vedi Leggi sulle - Ingegneria legale.

Problemi di Geometria elementare, dell'Ing. I. GHERSI, (Metodi facili per risolverli), con circa 200 problemi risolti, e 119 incisioni, di pag. XII-160, . . . . . 150

Procedura civile e procedura penale - vedi Codice.

Procedura privilegiata fiscale per la riscossione delle imposte dirette - vedi Esattore.

Processi fotomeccanici (I moderni). Fotocollografia, fototipografia, fotocalcografia, fotomodellatura, tricromia, del Prof. R. NAMIAS, p. VIU-316, 53 fig., 41 illustr. a 9 tav. 350 Prodotti agricoli del Tropico (Manuale pratico del piantatore), del Cav. A. GAsLINI. (II caffé, la canna da zuc- 
chero, il pepe, il tabacco, il cacao, il tè, il dattero, il cotone, il cocco, la coca, il baniano, l'aloè, l'indaco, il tamarindo, l'ananas, l'albero del chinino, la juta, il baobab, il papaia, l'albero del caoutchouc, la guttaperca, l'arancio, le perle). Di pag. XVI-270

Produzione e commercio del vino in Italia, di $\mathbf{S}$. MoNDINI, di pag. VII-303

Proftrmiere (Manuale del), di A. RossI, con 700 ricette pratiche, di pag. IV-476 e 58 incisioni

- vedi anche Ricettario domest. - Ricettario industr. - Saponi.

Prciezioni (Le). Materiali, Accessori, Vedute a movimento, Positive sul vetro, Proiezioni speciali policrome, stereoscopiche, panoramiche, didattiche, ece., del Dott. L. SASSI di pag. XVI-447, con 141 incisioni.

Proiezioni ortogonali - vedi Disegno.

Prontuario di geografia e statistica, del Prof. G. GAROLLO, pag. 62

Prontuario per lo paghe - vedi Paghe - Conti fatti.

Propriota letteraria, artistica e industriale - vedi Leggi.

Proprietario di case e di opifici. Imposta sui fabbricati, dell'Avv. G. GIORDANI, di pag. xx-264.

Prosodia - vedi Metrica dei greci e dei romani - Ritmica.

Prospettiva (Manuale di), dell'Ing. L. CLAUdr, $2^{\text {a }}$ edizione riveduta di pagine XI-61 con 28 tavole . . . . . . 2 Protezione degli animali (La), di NIGRo Licò, p. vIII-200 2 Protistologia, di L. MAGGI, ${ }^{2}$ ed., p. XVI-278, 93 incis. . . 3 Prołotipi (I) internazionali del metro e del kilogramma ed il codice metrico internazionale - vedi Metrologia.

Proverb: in 4 lingue - vedi Dottrina popolare.

Proverbi (516) sul cavallo, raccolti od annotati dal Colonnello VOLPINI, di pag. XIX-172

Psichiatra. Confini, cause e fenomeni della pazzia. Concetto, classificazione, forme cliniche o diagnosi delle materie mentali. Il manicomio, di J. FINZI, pag. vIII-225 . 250

Psisologia, del Prof. C. CANTonI, pag. VIII-168, 2a ediz. . 150 Psicologia fisiologica, del Dott. G. MANTovanI, pag. VIII-

165. con 16 incisioni. . . . . . . . . . . . 150

Psicologia musicale. Appunti, pensieri e discussioni, di M. PIL0 di pag. x-259. . . . . . . . , . . . . 250

Psicoterapia di G. PoRTIGLIOTTI, di pag. XII-318, 22 inc. 3 Pugilato e lotta per la difesa personale, Box inglese e francese, di A. CoUGNET, pag. XXIV-198, 104 incis. . 250 Raccoglitore (II) di J. GELLI (In lavoro).

Rachitide (La) e le deformità da esse prodotte, del Dott.

P. MANCINI. (In lavoro).

Radisgrafia - vedi Raggi Röntgen.

Radioterapia - vedi Luce e salute.

Ragioneria, del Prof. V. GImTr, $4^{\text {a }}$ edizione riveduta, di pag. VIII-141, con 2 tavole. 
Ragioneria delle cooperative di consumo (Mánuale di),

L.c. del Rag. G. RoTA, di pag. XV-408 . . . . . . . . $3-$

Ragioneria industriale, del Prof. Rag. OREste BergavaSCHI, di pag. VIr-280 e molti moduli . . . . . . . 3 -

Ragioniere (Prontuario del). (Manuale di calcolazioni mer-

cantili e bancarie), di E. GAGLIARDI, pag. XII-603 . .6 50 Ramatura - vedi Galvanostegia.

Razze bovine, equine, suine, ovine e caprine di F. FAELLI, di pag. XX-372, con 75 illustrazioni delle quali 16 colorate 550 Robus - vedi Enimmistica.

Reclami ferroviarii - vedi Trasporti e tariffe.

Fegiatro e Bollo - vedi Leggi sulle tasse di.

Regole calcolatore e sue applicazioni nelle operazioni topografiche, dell'Ing. G. POZZI, di pag. XV-238, con 182 incisioni e 1 tavola.

Religioni e lingue dell'India inglese, di R. Cust, tradotto dal Prof. A. DE GUBERNATIS, di pag. IV-124 . . . . 150 Resistenza dei materiali e stabilità delle costruzioni, di P. GallizIA, pag. X-336, con 236 inc, e 2 tavole. . . 550 Responsabilità - vedi Ingegneria legale.

Rettili - vedi Zoologia.

Rettorica, ad uso delle Scuole, di F. CAPELLO, p. VI-122. 150 Ribes - vedi Frutta minori.

Ricami - vedi Biancheria - Macchine da cucire - Monogrammi - Piccole industrie - Ricettario domestico - Trine.

Ricchezza mobile, dell'Avv. E. BRUNI, pag. vIII-218 . . 150 Ricettario domestico, dell'Ing. I. GHERSI. Adornamento della casa. Arti del disegno. Giardinaggio. Conservazione di animali, frutti, ortaggi, piante. Animali domestici e nocivi. Bevande. Sostanze alimentari. Combustibili e illuminazione. Detersione e lavatura. Smacchiatura. Vestiario. Profumeria e toeletta. Igiene e medicina. Mastici e plastica. Colle e gomme. Vernici ed encaustici. Metalli. Vetrerie, di pag. 550 con 2340 consigli pratici e ricette accuratamente scelte. . 550 Ricettario industriale, dell'Ing. I. GHERsI. Procedimenti utili nelle arti, industrie e mestieri, caratteri; saggio e conservazione delle sostanze naturali ed artificiali d'uso comune; colori, vernici, mastici, colle, inchiostri, gomma elastica, muterie tessili, carta, legno, fiammiferi, fuochi d'artificio, vetro; metalli, bronzatura, nichelatura, a rgentatura, doratura, galvanoplastica, incisione, tempera, leghe; filtrazione; materiali impermeabili, incombustibili, artificiali; cascami, olii, saponi, profumeria, tintoria, smacchiatura, inbianchimento; agricoltura, elettricità; $2^{a}$ edizione rifatta $e$ 
aumentata, di pag. VII-704, con 27 inc. e 2886 ricette Ricettario fotografico. Terza edizione riveduta e notevolmente ampliata di nuove formole e procedimenti, del Dott.

L. SASSI, di pag. XXIV-229.

Rilievi - vedi Cartografia - Compens. errori - Telemetria.

Risorgimento italiano (Storia del) 1814-1870, con l'aggiunta di un sommario degli eventi posteriori, del Prof. F. Bertolini, 2a ediz, di pag. VIII-208 . . . . . 150 Ristauratore dei dipinti, del Conte G. SECCO-SUARDo, 2 volumi, di pag. XVI-269, XII-362, con 47 incisioni. . . 6 Ritmica e metrica razionale italiana, del Prof. Rocco MURARI, di pag. XVI-216. . . . . . . . . . . 150 Rivoluzione francese (La) (1789-1799), del Prof. Dott. GIAN PAOLO SOLERIO, di pag. IV-176. . . . . . . . . 150 Roma antica - vedi Mitologia - Monete - Topografia.

Röntgen (I raggi di) e le loro pratiche applicazioni, di

ITALO TONTA, pag. VIII-160, con 65 incis e 14 tavole . 250 - vedi anche - Fototerapia e radioterapia.

Pihum - vedi Liquorista.

Saggiatore (Manuale del), di F. BUTTARI, di pag. VIII-245, con 28 incisioni.

Sale (II) e le Saline, di A. DE GASPARIS. (Processi indastriali, usi del sale, prodotti chimici, industria manifatturiera, industria agraria, il sale nell'economia pubblica e nella legislazione), di pag. VIII-358, con 24 incisioni . 350 Salumiere - vedi Majale.

Sanatorii - vedi Tisici e sanatorii.

Sanità e sicurezza pubblica. - Vedi Leggi sulla.

Sanscrito (Avviamento allo studio del), del Prof. F. G. FUMI,

$2^{a}$ edizione rifatta, di pag. $X \amalg-254$

Saponi (L'industria saponiera), con alcuni cenni sull'industria della soda e della potassa. Materia prima e fabbricazione in generale. Guida pratica dell'Ing. E. MARAZZA, (esaurito, è in lavoro la $2^{a}$ edizione).

Sarta da donna - vedi Abiti - Biancheria.

Scacchi (Manuale del ginochi degli), di A. SEGHIERI, $2^{2}$ ediz. ampliato da E, OrsinI, con una appendice alla sezione delle partite giuocate e una nuova raccolta di 52 problemi di antori ital., di pag. VI-310, con 191 incis. . 3Scaldamento e ventilazione degli ambienti abitati, di $R$. FERRINI, 2a ediz., di pag. VIII-300, con 98 incisioni. . . 3 Scenografia (La). Cenni storici dall'evo classico ai nostri giorni, di G. FERRARI, di pag. XXIV-327, con 16 incisioni nel testo, 160 tavole e 5 tricromie. 
Scherma italiana di J. GELLI, $2^{3}$ ediz., di pagine VI-25̃1,

\section{L c} con 108 figure

Sclarado - vedi Enimmistica.

Scienza delle finanze, di T. CARnevaLI, pag. IV-140 . . 150 Scritture d'affari (Precetti ed esempi di), per uso delle Scuole tecniche, popolari e commerciali, del Prof. D. MAF-

FIOLI, $3^{a}$ ediz. ampliata e corretta, di pag. VIII-221 . . 150 Sconti - vedi Interesse e sconto.

Scoperte geografiche - vedi Cronologia.

Scultura italiana antica e moderna (Manuale di). dell'Arch.

A. MELANI. $2^{\text {a }}$ edizione rifatta con 24 incisioni nel testo

e 100 tayole, di pag. XVII-248

Scuole industriali - vedi Industrie (Piccole).

Segretario comunale - vedi Esattore.

Selvicoltura, di A. SANTILLI, di pag. vщ-220, e 46 inc. . 2 -

Semeiotica. Breve compendio dei metodi fisici di esame

degli infermi, di U. GABBI, di pag. XVI-216, con 11 inc. 250

Sericoltura - vedi Bachi da seta - Filatura - Gelsicultura Industria della seta - Tintura della seta.

Servitù - vedi Ingegneria legale.

Shakespeare, di DowDEN, trad. di A. BALZANI, p. XII-242 150 Seta (Industria della), del Prof. L. GABBA, 2a ed., p. IV-208 2 Seta artificiale - vedi Imitazioni.

Sicurezza pubblica - vedi Leggi di sanità.

Siderurgia (Manuale di), dell'Ing. V. ZoPPETr, pubblicato e completato per cura dell'Ing. E. GARUFFA, di pag. IV368 , con 220 incisioni.

Sieroterapia, del Dott. E. ReBuschinI, di pag. VIII-424 . 3 Sigle epigrafiche - vedi Dizionario di abbleviature.

Sinistri marittimi - vedi Liquidatore di avarie.

Sintassi francese, razionale pratica, arricchita della parte storico-etimologica, della metrica, della fraseologia commerciale, ecc., del Prof. D. RODARI, di pag. XVI-206 . 150 - (Esercizi relativi alla) del Prof. D. RODARI. (in lavoro). Sintassi latina, di T. G. PERASSI, di pag. VII-168 . . . 150 Sismologia, del Capitano L. GATTA, di pag. VIII-175, con 16 incisioni $\theta 1$ carta. Smacchiature - vadi Ricettario domestico.

Smaiti - vedi Amatore d'oggetti d'arte - Fotosmaltografia Ricettario industr.

Soccorsí d'urgenza, del Dott. C. Calliano, $4^{a}$ edizione riveduta ed ampliata, di pag. XLVI-352, con 6 tav. litogr. Socialismo, di G. BIRAGHI, di pag. XV-285.

Società di mutuo soccorso. Norme per l'assicurazione delle pensioni e dei susssidi per malattia e per morte, del Dott. G. GardengHI, di pag. vI-152. . . . . . . 150 Società industriali italiane per azioni, del Dott. F. PICCINELII, di pag. XXXVI-534. 
Sociologia generale (Elementi di), del Dott. EMmLio MorSELLY, di pag. XII-172. . . . . . . . . . 150

Sofisticazione e adulterazione del vino e dell'aceto di A. ALOI (In lavoro),

Sordomuto (II) e la sua istruzione. Manuale per gli allievi e le allieve delle $\mathrm{R}$. Scnole normali, maestri e genitori, del Prof. F. FORNARI, di pag. VIII-232, coe 11 inc. 2 - vedi anche Ortofrenia.

Sostanze alimentari. - vedi Conservazione delle.

Specchi (La fabbricazione degli) e la decorazione del vetro e cristallo, del Professor R. NAMIAs, di pagine x $\mathrm{x}-156$, con 14 incisioni

Spettrofotometria (La) applicata alla Chimica fisiologica, alla Clinica e alla Medicina legale. Studio critico sperimentale di G. Gallerani, di pag. XIX-395, con 92 incisioni e tre tavole

Spettroseopio (Lo) e le sue applicazioni, di R. A. PROCTOR, traduzione con note ed aggiunte di F. PORfo, di pag. VI-179, con 71 incis. e una carta di spettri

Spiritismo, di A. PapPalardo, Seconda edizione, con 9 tavole, di pag. XVI-216

- vedi anche Magnetismo - Telepatia.

Spirito di vino - wi Alcool - Cognac - Distillaz. - Liquorista. Stagno (Vasellame di) - vedi Amatore di oggetti d'arte e di curiosità - Leghe metalliche.

Statica - vedi Metrologia - Strumenti metrici.

Statistica, del Pr. F. VIRGILI, $3^{2}$ ed., rifatta pag. XIX-225 150 Stearineria (L'industria stearica). Manuale pratico dell'Ing. E. MARAZZA, di pagine XI-284, con 70 incisioni e molte tabelle

Stello - vedi Astronomia - Cosmografia - Gravitazione Spettroscopio.

Stemmi - vedi Araldica - Numismatica - Vocabol. araldico.

Stenografia, di G. GIORGETTI, (secondo il sistema Gabelsberger-Noe), $2^{a}$ ediz., (esaurito, è in lavoro la $3^{2}$ ediz.). Stenografia (Guida per lo studio della) sistema Gabelsberger-Noe, compilata in 35 lezioni da A. NICOLETTI, $3^{2}$ ed. riveduta, di pag. VIII-160.

Stenografia. Esercizi graduali di lettura e di scrittura stenografica (sistema Gabelsberger-Noe), con 3 novelle del Prof. A. NIColetTi, $2^{a}$ ediz., di pag. VIII-160 . . . 150 - vedi anche Antologia stenografica - Dizionario stenografico.

Stenografo pratico (Lo) di L. CRISTOFoLI, di pag. XII-131 150 Stereometria applicata allo sviluppo dei solidi e alla loro costruzione in carta, del Prof. A. RiveLLI, di pag. 90, con 92 incisioni e 41 tavole. 
Stilistica, del Prof. F. CAPELLO, di pag. XII-164. . . . 150

Stilistica latina, di A. BARToLI. (In lavoro).

Stimatore d'arte - vedi Amatore di oggetti d'arte e di curiosità - Amatore di maioliche e porcellane - Armi antiche

Storia antica. Vol. I. L'Oriente Antico, del Prof. 1. GENTHL, di pag. XII-232 . . . . . . . . . . . 150

Vol. II. La Grecia, di G. TonIAzzo, pag. IV-216 . . 150 Storia dell'Arte, del Dott. G. CARotri. (In Iavoro). Storia dell'arte militare antica e moderna, del Cap. V. Rossetwo, con 17 tav. illustr., di pag. vIII-504. • . . 550 - vedi anshe Armi antiche.

Storia e cronologia medioevale moderna, in CC tavole sinottiche, del Prof. V. Casagrand, $3^{\text {a }}$ edizione, con nuove correzioni ed aggiunte, di pag. vIII-254 . . . 150 Storia della gimnastica. - Vedi Ginnastica.

Storia d'Italia (Breve), di P. ORsI, 2a ed. rived, p. XII-276 . 150 Storia di Francia, dai tempi più remoti ai giorni nostri, di G. BRAGAGNoLo, di pag. XVI-424, con tabelle cronologiche e genealogiche.

Storia ital. (Man. di), di C. CANTÙ, pag. IV-160 (esaurita). Storia d'Inghilterra dai tempi più remoti ai giorni nostri, del Prof. G. Bragagnolo, di pag. XVI-367 . . . . . 3 Storia della musica, del Dott. UNTERSTEINER, $2^{a}$ edizione ampliata, di pag. XII-330. . . . . . . . . . 3 Strumentazione, per E. PRouT versione italiana con note di V. RICCI, $2^{\text {a }}$ ediz. rived., di p. XVI-214, 95 incis. . . . 250 Strumenti ad arco (Gli) e la musica da camera, del Duca di Caffarelli, di pag. $\mathrm{X}-235$. . . . . . . . 250 Strumenti metrici (Principî di statica e loro applicazione alla teoria e costruzione degli), dell'Ing. E. BAGNorr, di pag. VIII-252, con 192 incisioni . . . . . . . 350 Stufe - vedi scaldamento.

Suono - vedi Luce e suopo.

Succedanei - vedi Ricettario industriale - Imitazioni.

Sughero - vedi Imitazioni e succedanei.

Surrogati - vedi Ricettario industriale - Imitazioni.

Sussidi - vedi società di mutuo soccorso.

Tabacco, del Prof. G. CANTONI, di pas. IV-176, con 6 inc. 2 Tabacchiere artistiche - vedi Amatore di oggetti d'arte.

Tacheometria - vedi Celerimensura - Telemetria - Topografia

- Triangolazioni.

Tamarindo - vedi Prodotti agricoli.

Tappezzerie - vedi Amatore di oggetti d'arte e di cnriusità.

Tariffe ferroviarie - vedi Codice dog. - Trasporti e tariffe. 
Tartufi (I) ed i funghi, loro natura, storia, coltura, conser-

vazione e cucinatura, di FOLCO BRUNI, di pag. VII-184 2 Tasso di registro, bollo, ecc. - vedi Codice di bollo - Esattore - Imposte - Leggi Tasse Reg. e Bollo - Notaio - Ricch. mob. Tassidermista - vedi Imbalsamatare - Naturalista viaggiatore. Tatuaggio - vedi Chiromanzia e tatuaggio.

Tò - vedi Prodotti agricoli.

Teatro - vedi Letteratura drammatica - Codice del teatro.

Tecrica microscopica - vedi Anatomia microscopica.

Tavole d'alligazione per. l'oro e per l'argento con nume-

rosi es. pratici per il loro nso, F. BUTTARI, p. XII-220. 250 Tavole logaritmiche - vedi Logaritmi.

Tavole schematiche della Divina Commedia di Dante Alighieri, di L. PoLACCO, seguite da sei tavole topogr. in eromolit. disegn. dal Maestro G. AGNELLI, pag. X-152 . 3 Tecnica protistologica, del Prof. L. MAGGI, pag. XVI-318 3 Tocnologia - vedi Dizionario tecnico.

Tecnologia meccanica - vedi Modellatore meccanico.

Tecnologia e terminologia monetaria, di G. SACCHETTI, di pag. XVI-191

Telefono, di D. V. PrccolI, di p.IV-120, con 38 incis., L. 2. (Esauritı, è in lav. la $2^{a}$ ediz. complet. rifatta da G. MotTA). Telegrafia, del Prof. R. FERRINI, $2^{a}$ edizione corretta ed accresciuta, di pag. VIII-315, con 104 incisioni . . . . 2 -

Telegrafo senza fili e Onde Hertziane di O. Muranr, di pag. $\mathrm{xv}-341$, con 172 in cisioni . . . . . . . . . 350

Telemetria, misura delle distanze in guerra, del Cap. G. BERTELLI, di pag. XIII-145, con 12 zincotipie . . . . 2 Telepatia (Trasmissione del pensiero), di A. PAPPaLARDo, $2^{a}$ ediz. di pag. XVI-279

- vedi anche Magnetismo - Ipnotismo - Spiritismo.

Tempera e cementazione, dell'Ingegner FADDA, di pagine VIII-108, con 20 incision.

Teoria dei numeri (Primi elementi della), per il Prof. U. SCARPIS, di pag. vIII-152 . . . . . . . . . . . 150 Teoria delle ombre, con un cenno sul Chiaroscuro e sul colore dei corpi, del Prof. E. BoNCr, di pag. VIII-164, con 36 tavole e 62 figure . . . . . . . . . . . 2 Terapia delle malattie dell'infanzia, del Dott. G. CATTANEO, di pag. XII-506 . . . . . . . . . 4 -

Termodinamica, Prof. G. Cattaneo, pag. X-196, 4 fig. . 150 Terremoti - vedi Sismologia - Vulcanismo.

Torreni - vedi Chimica agraria - Concimi - Humus.

Tessitore (Manuale del), del Prof. P. Pinchetti, 2a edi\%. riveduta, di pag. XvI-312, con illustrazioni 
Tessuti di lana e di cotone (Analisi e fabbricazione dei). Manuale pratico razíonale di 0 . GrudrcI, di pag. XII-864 con 1098 incisioni colorate . . . . . . . . . . 1650

Testamenti (Manuali dei), per cura del Dott. G. SERINA, di pag. vI-238 . . . . . . . . . . . . . . 250

Tigrè-italiano (Manuale), con due dizionarietti italiano-tigrè e tigrè-italiano ed una cartina dimostrativa degli idiomi parlati in Eritrea, del Cap. M. CAMPERIO, di pag. 180 . 250

Tintore (Manuale del), di R. LEPETIT, $3^{a}$ edizione, di pagine $X-279$, con 14 incisioni.

Tintura della seta, studio chimico tecnico, di T. PASCAL, di pag. XVI-432

Tipografia (Vol. I). Guida per chi stampa e fa stampare. Compositori, Correttori, Revisori, Autori ed Editori, di S. LANDI, di pag. 280 . . . . . . . . . . . . 250

Tipografia (Vol. II). Lezioni di composizione ad uso degli allievi e di quanti fanno stampare, di S. LANDI, di p. virI271 , corredato di figure e di modelli.

- vedi anche Vocabolario tipografico.

Tisici e i Sanatorii (La cura razionale dei), del Dott. A. ZUBIANI, prefaz. del Prof. B. SILVA, p. XLI-240, 4 incis. 2 ritoli di rendita - vedi Debito pubblico - Valori pubblici.

Topografia e rilievi - vedi Cartografia - Catasto - Celerimensura - Compensazione errori - Curve - Disegno topografico - Estimo terreni - Estimo rurale - Fotogrammetria - Geoınetria pratica - Prospettiva - Regolo calcolatore - Telemetria - Triangolazioni.

Topografia di Roma antica, di L. BoRSARI, di pag. VIII436, con 7 tavole

Tornitore meccanico (Guida pratica del), ovvero sistema nnico per calcoli in generale sulla costruzione di viti e ruote dentate, arricchita di oltre 100 problemi risolti, di S. DrNaro, $2^{\text {a }}$ edizione, di pag. XIr-175

Traduttore tedesco (Il), coznpendio delle principali diffcoltà grammaticali della Lingua Tedesca. del Prof. $R$. MINUTrTI, di pag. XVI-224.

Trasporti, tariffe, reclami ferroviari ed operazioni doganali. Manuale pratico ad uso dei commercianti e privati, colle norme per l'interpretazione delle tariffe vigenti, di A. Branchi $2^{2}$ edizione rifatta. di pagine XVI-208.

Travi metallici composti - Vedi Momenti resistenti.

Triangoiazioni topografiche e triangolazioni catastali, dell'Ing. O. JACOANGELI. Modo di fondarle sulla rete geodetica, di rilevarle e calcolarle, di pag. XIV-340, con 32 
incisioni, 4 quadri degli elementi geodetici, 32 modelli pei ealcoli trigonometrici e tavole ausiliarie . . . . 750 Trigonometria - vedi Celerimensura - Esercizi Geometria metrica - Geometria metrica - Logaritmi.

Trigonometria della sfera - vedi Geometria e trigonom. della.

Trine (Le) a fuselli in Italia. Loro origine discussione, confronti, cenni bibliografici, analisi, divisione, istruzioni tecnico-pratiche con 200 illustrazioni intercalate nel testo di GIACINTA ROMANELLI-MARONE, di pag. VIII-331 . . 450 Jubercolosi - vedi Tisici.

Uccelli canori (I nostri migliori); loro caratteri e costumi. Modo di abituarli e conservarli in schiavitù. Cura delle Joro infermità. Maniera per ottenere la riproduzione del Canarino, di L. UNTERSTEINER, di pag. XU-175 . . . 2 Ufficiale (Manuale per l') del Regio Esercito italiano, di U. MORINI, di pag. XX-388 . . . . . . . . . 350 Ufficiaie sanitario (Manuale dell') di C. TONZIG e G. RUATA (In lavoro).

Unità assolute. Definizione, Dimensioni, Rappresentazione.

Problemi dell'Ing. G. BERTOLINI, pag. X-121. . . . 250 Usciere - vedi Conciliatore.

Uva spina - vedi Frutta minori.

Uve da tavola. Varietà, coltivazione e commercio, del Dott.

D. TAMARO, $3^{\mathrm{a}}$ edizione, di pag. XVI-278, con tavole colorate, 7 fototipie e 57 incisioni . . . . . . . 4 Valli lombarde - vedi Dizionario alpino - Prealpi Bergamasche. Valori pubblici (Manuale per l'apprezzamento dei), e per le operazioni di Borsa, del D. F. PICCINELLI, $2^{\mathrm{a}}$ edizione rifatta e accresciuta, di pag. XXIV-902 . . . . . 750 Valutazioni - vedi Prontuario del ragioniere.

Vasellame antico - vedi Amatore di oggetti d'arte e curiosità.

Veleni ed avvelenamenti, dėl Dott. C. FERRARIs, di pag.

XVI-208, con 20 incisioni.

Velocipedi - vedi Ciclista.

Ventagli artistici - vedi Amatore di oggetti d'arte e di curiosità. Ventilaziono - vedi Scaldamento.

Verbi greci anomali (I), del Prof. P. SPAGNotTi, secondo le Grammatiche di CuRTIUs e INAMA, pag. XXIV-107 . 150 Verbi latini di forma particolare nel perfetto e nel supino, di A. F. Pavanello, con indice alfabetico di dette forme, di pag. VI-215.

Vermouth - vedi Liquorista.

Vernici (Fabbricazione delle), e prodotti affini, lacche, mastici, inchiostri da stampa. ceralacche, dell'Ing. UGO FORNARI, $2^{2}$ ediz. riveduta e ampliata di pag. XII-244.2 Vetri artistici - vedi Amatore oggetti d'arte - Specchi - Fotosmaltografia. 
Vetro (II). Fabbricazione, lavorazione meccanica, applicazioni alle costruzioni, alle arti ed alle industrie, dell'ing. G. D'ANGELO di pag. XIX-527, con 325 figure intercalate, delle quali 25 in tricromia.

Vini blanchi da pasto a Vini mezzo colore (Guida pratica per la fabbricazione, l'affinamento e la conservazione dei), di G. A. Prato, di pag. XII-276, con 40 inc.

Vino (II), di G. GrasSI-SONCINI, di pag. XVI-152.

Vino aromatizzato - vedi Cognac - Liquorista.

Viticoltura. Precetti ad nso dei Viticoltori italiani, del Prof. O. OTTAVI, $5^{\text {a }}$ ed. riveduta ed ampliata da A. STRUCCHI, di pag. XVI-227, con 30 incisioni .

Yocabolarietto pei numismatici (in 7 lingue), del Dott. S. AMBROsOLI, di pag. VIII-134

Vocabolario araldico ad uso degli italiani, del Conte G. GUELFI, di pag. VIII-294, con 356 incisioni . . . .

Yocabolario compendioso della lingua russa, del Prof. VoIvovICH, di pag. XVI-238

Vocabolario tipografico, di S. LANDI. (In lavoro).

Volapiuk (Dizionario italiano-volapük), preceduto dalle Nozioni compendiose di grammatica della lingua, del Prof. C. MATTEI, secondo i principii dell'inventore M. SCHLEYER, ed a norma del Dizionario Volapík ad uso dei francesi, del Prof. Kerckhoffs, di pag. $\mathrm{XxX}-198$. . . . . 250

Volapük (Dizion. volapük-ital.), Prof. C. MATTEI, p. XX-204. 250 Volapük, Manuale di conversazione e raccolta di vocaboli e dialoghi italiani-volapïk, per eura di M. RosA, TOMMASI e A. ZAMBELLI, di pag. 152. . . . . . . . 250 Vulcanismo, del Cap. L. GATTA, di pag. VIII-268 e 28 inc. . 150 Zecche - vedi Terminologia monetaria.

Zolfu (Le miniere di) di G. CAGNI. di pag. XII-275, con 34 incisioni e 10 tabelle... . . . . . . . . 350 Zoologia, dei Prof. E. H. Giglioli e G. Cavanna:

1. Invertebrati, di pag. 200 , con 45 figure . . . . 150

II. Vertebrati, Parte I, Generalita, Ittiopsidi (Pesci ed Anfibi), di pag. XVI-156, con 33 incisioni

III. Vertebrati. Parte II, Sauropsidi, Teriopsidi (Rettili, Uecelli e Mammiferi), di pag. XVI-200, con 22 incis.. 150 Zoonosi, del Dott. B. CALLI VALERIO, di pag. XV-227. . 150 Zootecnia, del Prof. G. TAMPELINI, p. VIII-297, 52 incis. 250 Zucchero (Industria dello):

I. Coltivazione della barbabietola da zucchero, dell'Ing.

B. R. DEBARBIERI, di pag. XVI-220, con 12 inc.. . 250

II. Commercio, importanza economica e legislazione doganale, di L. FoNTANA-Russo, di pag. XII-244 . 250 III. Fabbricazione dello zucchero di barbabietola del-

l'Ing. A. TACCANI, di pag. XII-228, con 71 incis . . 350 


\section{Indice alfabetico per autori dei Manuali Hoepll}

(I numeri indicano le pagine).

Abbo P. Nuotatore.......444

Acqua C. Nicroscopio ...... 42 Adter G. Esere. di lingua tedesca 28 Aducco A. Chimica agraria. . . 17 Agnelli 6. Tav. Div. Commedia 54 Airy G. B. Gravitazione..... 34 Alasia C. Eserc. Trigon. piana 28 - Complementi di geomet. elem. 20 - Geometria della sfera ... 32 Alberti F. Il bestiame el'agricol. 15 Albicini G. Diritto civile . . . 23 Albini G. Fisiologia. ...... . 29 Alessandri P.E. Analisi chimica 11 - Analisi volumetrica..... 11

- Chimica applic. all'Igiene. 17

- Disinfezione......... 24

- Farmacista (Manuale del). 29

- Sostanze alimentari.... 5 Allori A. Dizionario Eritreo . . 25 Aloi A. Olivo ed olio ..... 44 - Agrumi ........... 10 - Sofisticazione vino .... . 52 Ambrosoli s. Atene....... 14 - Monete greche . . . . . . 43

- Numismatica ...... . . 44

- Vocabularietto pei numism. 57 Antilli A. Disegno geometrico. 24 Antonini E. Pellagra ......46 Appiani G. Colori e vernici . 19 Arlia C. Dizionario bibliogr. . 25 Arrighi C. Dizionario rílanese 25 Arti grafiche, ecc. ........ 13 Aschieri F. Geom.anal. d.spazio 32 - Geometria anal. del piano 32

- Geometria descrittiva . . 32

- Geom. projettiva del piano e della stella.

Geom. projett. dello spazio 32

Azimonti E. Frumento . . . . . 31 - Campicello scolastico . . . 16

- II ais ............ . 40

Azzoni F. Debito pubb. italiano 23 Baccarini P. Malatt. crittogam. 40 Baddeley v. Law-Tennis . . . . 37 Bagnoli E. Statica ....... 53 Ball J. AIpi (Le). . . . . . . . . 11 Ball $R$ Staweil. Meccanica ...4 41 Ballerini 0. Fiori artiflciali . . 29 Balzani A. Shakespeare..... 51 Baroschi E. Fraseologia franc. 30 Barpi U. Igiene veterinaria . 34 - Abitaz. degli anim. domest. 10 Barth M. Analisi del vino ... 11 Bartoli A. Stilistica latina ... 53 Bassi D. Mitologie orientali. 43 Bastiani F. Lavori marittimi . 36
Belifore G. Magnet. ed ipnot . 40 Bellini A. Igiene della pelle. . 34 - Luce e salute, kototerapia

e radioterapia. . . . . . 39

Bellio V. Mare (Il) . . . . . . . 41

- Cristoforo colorubo . . . . 22

Bellotti G. Luce e colori ... . . 39

Belluomini G. Calderaio prat . 16

- Cubatura dei legnami . . .22

- Fabbro ferraio...... . ¿8

- Falegname ed ebanista .. 28

- Fonditore. . . . . . . . . 30

- Operaio (Manuale dell') . . 44

- Peso dei metalli .......446

Beltrami L. Aless Manzoni .. 41

Benetti J. Mecuanica...... 41

Bergamaschi 0 . Contabilità dom. 20 - Ragioneria industriale . . 49 Bernardi G. Armonia ...... 13 Bernhard. Infortunii di mont. . 36 Bertolli G. Disegno topografico 24 - Telemetria.........54 Bertolini F. Risorg. italiano . . 50 Bertolini G. Unità assolute. . .56 Bertalio 5 . Coltiv. delle min. . 42 Besta R. Anat. e fisiol. compar. 11 Bettel v. Morfologia greca. . . 43 Bettoni E. Piscicoltura. . . . 46 Biagi G. Bibliotec. (Man. del). 15 Bianchi A. G. Trasporti e tariffe ferroviarie ......... 55 Bignami-Sormani E. Dizionario alpino italiano....... 26 Biraghi G. Socialismo ..... 51 Bisconti A. Esercizi greci .. 28 Boccardini G.L'Euclide emendato 28 Bock C. Igiene privata .... . 34 Boito C. Disegno (Princ. del) 24 Bombici C. Mineral. generale 42 - Mineralogia descrittiva. . 42 Bonacini C. Fotografia ortocr. 30 Bonci E. Teoria delle ombre . 54 Bonelli L. Grammatica turca , 34 Bonettl E. Biancheria (Disegno,

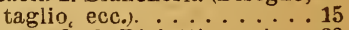
Bonine G. B. Dialetti greci. . . 23 Bonizzi P. Animali da cortile. 12 - Colombi domestici .... 19 Borgarello E. Gastronomia, Terninologia italiana e franc. 31 Borletti F. Celerimensura. . . 17 - Form. per il calc. d. risvolte 30 Borsari L. Topog. di Roma ant. 55 Boselli E. Gioielleria e orefic. 32 Bossi L. M. Ostetricia..... 45 Eragagnolo G. Storia di Francia 53 
Bragagnolo G. Storia d'Inghilt. 53 Brigiuti L. Letterat. egiziana. 38 Brocherel E. Alpinismo......11 Brown H. T. Meccanismi $(500) 41$ Bruni F. Tartufi e funghi . . 54 Bruni E. Catasto italiano. . . 17 - Codice doganale italiano.. 18

- Contabilità dello Stato. . 21

- Imposte dirette. ....... 35

- Legislazione rurale . . . . . 3 37

- Ricchezza mobile ..... 49

Bruttini A. Il libro dell'agricol. 10

Bucci di Santafiora. Marino ...41

- Flotte moderne (Le). . . . 29

Budan E. Autografi (Amat. di). 14 Burali-Forti C. Logica matem. 39 Buttari F. Sagyiatore (Man. d.) 50 - Tav. per l'allig. oro e arg. 54 Caffarelli $F$. Strumenti ad arco 53 Cagni G. Le miniere di zolfo.. 57 Calliano C. Soccorsi d'urgenza 51 - Assistenza degli infermi. . 13 Calzavara V. Industria del gas 31 Camperio $M$. Tigrè-italiano .. 55 Campl C. Campicello scolastico 16 Canestrini E. Fulmini e paraf. 31 Canestrini G. Apicoltura. . . . 12 - Antropologia

Canestrini C. e R. Batteriologia 15

Cantamessa F. Alcool. . . . . . 10

Cantoni C. Logica. . . . . . . . . 39

Cantoni c. Psicologia . . . . . 48

Gantoni G Prato (ii) . . . . . . 47

- Tabacco (Il) . . . . . . . . 53

Cantoni P. Igroseopi, igrometri, umidita atmosferica. . . 35

Cantü C. Storia italiana ....53

Capello F. Rettorica. . . . . . 49

- Stilistica .......... 53

Capilupi A. Assicuraz. e stima 13 Capelletti L. Napoleone I. . . 44 - Letterat. snagn. e portogh. 38 Cappelletti L. Nevrastenia ... 44 Cappelli A. Diz. di abbreviat. . 25 Carazzi D. Ostricoltura.....45 - Anat. microsc. (Tecn. di) 11 Carega di Muricce. Agronomia 10 - Estimo rurale. . . . . . 28 Carnevali $T$. Scienza finanze . 51 Carotti S. Storia dell'arte ...53 Carraroli A. Igiene rurale. . . 34 Casagrandi V. Storia e cronol. 53 Casali A. Humus (L'). . . . . 34 Castellani L. Acetilene (J'). . . 10 - Incandescenza........ 35

Castiglionl L. Beneficenza ... 15 Cattaneo C. Dinamica element. 23
Cattaneo C. Terapia infanzia . 54 Gattaneo G. Embriolog. e morf. 27 Cavaíieri D. Legisl. delle acque 37 Cavanna G. Zoologia...... 57 Cavara F. Funghi mangerecci. 31 Celoria G. Astronomia...... 14 Cencelli-Perti A. Macch. agric. 39 Cerchiari G. L. Chiromanzia e tatuaggio ........... 18 Cereti P. E. Esercizi latini. . . 28 Cerruti F. Meccanismi (500) . . 41 Cerutti A. Fognat. domestica . 30 Cettolini S. Malattie dei vini . 40 Ciapetti G. L'alcool industriale 10 Chiesa C. Logismografia . . . 39 Ciampoli D. Letterature slave. 38 Cignoni A. Ingegnere navale . 36 Claudi C. Prospettiva ......48 Clerico G. vedi Müller, Metrica 42 Collamarini G. Biologia ..... 15 Colombo G. Ingegnere civile. . 36 Colombo L. Nutriz. del Bamb. 44 Comboni E. Analisi del vino. . 11 Concari T. Gramm. italiaua . . 33 Consoli S. Fonologia latina.. 30 - Letteratura norvegiana . . 38 Conti P. Giardino infantile . . 32 Contuzzi F. P. Diritto costituz. 28 - Diritto internaz. privato. . 24 - Diritto internaz. pubblico. 24 Corsi E. Codice del bollo.... 18 Cossa A. Elettrochimica . . . . 26 Cossa L. Economia politica .. 26 Cougnet. Pugilato antico e mod. 48 Coulliaux L. Igiene della bocca. 34 Gova E. Confez. abiti signora. 10 Cremona 1. Alpi (Le) ...... 11 Cristofoli L. Stenografo pratico 52 Crollalanza G. Araldica (Gr.). . 12 Croppi G. Canottaggio. ..... 16 Crotti F. Compens. degli errori 20 Curti $R$. Infortuni della mont. 36 Cust R. Relig. e lingue d. India 49 - Lingue d'Africa ..... 39 D'Adda L. Marine da guerra . 41 Dal Piaz. Cognac . . . . . . 19 Damiani Lingue straniere . . . 39 D'Angelo s. Vetro. . . . . . 57 Da Ponte M. Distillazione . . 24 De Amezaga. Marino militare 41 De Barbleri R. Zucchero (Ind.d.) 57 De Brun A. Contab. comunale. 20 De Cillis E. Mosti (Densità dei) 44 Do Gasparis A. Sale e Saline . 50 De Gregorio G. Glottologia... 33 De Gubernatis A. Lett. indiana 38 - Lingue d'Afr. . . . . . . 39 - Mitologia comparata . . . 43 
De Gubernatis A. helig e lingue dell'Indıa.

Del!'Acqua F.Morte vera e appar.

Del Lupo \%. Pomol. artificiale. 47

De Miarchi L. Meteorologia. . . 42

- Climatologia......... 18

De Mauri L. Maioliche(Amatore di) ............ 40

- Amature d'oggetti d'arte . 11

Dessy. Elettrotecnica . . . . . 27

Di Maio F. Pirotecnica. . . . . 46

Dinaro $S$. Tornitore meccanico 56 - Montatore di macchine . . 43

- Atlante di macchine . . . 39

Dizionario universale in 4 lingue 26

Dompè C. Man. del Commerciante 20

Dowden. Shakespeare . . . . . 51

Doyen C. Litografia. . . . . . . 39

Enciclopedia Hoepli.. . . . . . 27

Erede G. Geometria pratica. . 32

Fabris 6 . Olii vegetali......44

Fadia. Tempera e cementaz. . 54

Faelli F. Razze equine . . . . 49

Falcone C. Anat. topografica 12

Faralli G. Ig. della vita pub. e pr. 34

Fenini C. Letteratura italiana 38

Fenizla c. Evoluzione..... 28

Ferrari D. Arte ( $L$ ') del dire...3

Ferrari G. Scenografia (La) .. 50

Ferrari v. Lett. moderna ital. 38

- Letter. moderna e contemp. 38

Ferrario C. Curve circolari. .. 23

Ferraris c. Veleni ed avvelen. 56

Ferrini c. Digesto (II) . . . . . 23

- Diritto penale romano. .. 24

- Diritto romano... . . . . 24

Ferrini R. Elettricità . . . . 26

- Energia fisica. . . . . . 27

- Galvanoplastica. . . . . . 31

- Scaldamento e ventilaz.. .50

- Telegrafia ......... 54

Filippini P. Estimo dei terreni 28

Finzi J. Psichiatria....... 48

Fiorilli c. Omero ......... 44

Fiori A. Dizionario tedesco . . 26 - Conversazione tedesca . . . 21

Fontana-Russo. Zucch. (Comm.) 57

Foresti A. Mitologia greca. .. 43

Formentano A. Giurisdizione volontaria............ 33

Formenti c. Alluminio ....... 11

Fornari P. Sordomuto (Il) . . 52

Fornari U. Vernici e lacche ..56

- Luce e suouo ........ 39

- Calore (II) . . . . . . . 16

Foster M. Fisiologia . . . . . 29

Franceschi G. Cacciatore....15

- Corse
Franceschig Giunco del Pallone 33

Franceschi G. B. Concia pelli . 20

- Conserve alimentari. . . . 20

Franceschini F. Insetti utili . . 36

- Insetti nocivi ....... 36

Franchi L. Codici . . . . 18-19

- Leggi sui lavori pubblici . 37

- Legge s. tasse di reg. e bollo 37

- Legge sull'Ordin. giudiz. . 37

- Legge sanità e sicur, pubbl. 37

- Leggi sulle privat. industr. 37

- Leggi diritti d'autore 18-19-37

Friedmann S. Lingua gotica . . 38

Friso L. Filosofia morale. . . 29

Frisoni G. Gramm. port.-bras. 34 - Corrispondenza italiana.. 21

- $\quad$ spagnuola 22

- francese..22

- Gramm. Danese-Norveg.. . 33

Fumagalli G. Bibliotecario .... 15

- Paleografia........ 45

Fumi F. Q. Sanscrito. . . . 50

Funaro A. Concimi (I) . . . 20

Gabba L. Chimico (Man. del) 17.

- Seta (Industria della). . . 51

- Adult. e falsific. degli alim. 10

Gabbi U. Semeiotica.......51

Gabelsberger-Noë. Stenografia. 52

Gabrielli F. Giuochi ginnastici 33

Gagliardi E. Interesse e sconto 36

- Ragionier e Pront. del). . . 49

Galassini A. Maec. cuv. e ricam. 40

Gallerani G. Spettrofotometria 52

Galletti E. Geografia....... 31

Galli G. Igiene privata ....34

Galli Valerio B. Zoonosi . . . . 5 57

- Immunità e resist. alle mal. 35

Gallizia P. Resistenza dei mater. 49

Gardenghi G. Soc. di mutuo soc.. 51

Garetti A. Notaio (Man del). . 44

Gardini A. Chirurgia operat $\ldots 18$

Garibaldi c. Econ. matematica 26

Garnier-Valletti Pomologia . . 47

Garollo G. Atl. geog.-st. d'Ital. 14

- Dizionario biograf. univ. . 25

- Dizionario geograf. univ. . 25

- Prontuario di geografia.. 48

Garuffa E. Orologeria ..... 45

- Siderurgia ......... 51

Gaslini A. Prodotti del Tropico 47

Gatia L. Sismologia ......5 51

- Vulcanismo ........ 57

Gautero G. Macch. e fuochista 40

Gavina F. Ballo (Manuale del). 14

Geikle A. Geografia fisioa. . . 32

- Geologia.......... 32

Golcich E. Cartografia. . . . . 17 
Gelli J. Armi antiche

13

- Biliardo. ......... 15

- Codice cavalleresco....18

- Dizionario filatelico..... 25

- Duellante.......... 26

- Ginnastica maschile. . . . 32

- Scherma ..........51

- Il raccoglitore ......... 48

Gentilo 1. Archeologia deli'arte 12

- Geografia classica..... 27

- Storia antica (Oriente). . . 53

Gernesio Q. Imitaz. di Cristo . 35

Gestro R. Natural. viaggiat.. . 44 - Naturalista preparatore..44

Ghersi 1. Ciclista ........ 18

- Conti fatti.........21

-. Galvanostegia. . . . . . . . 31

- Imitazioni e succedanei . . 35

- Industrie (Piccole). . . . . 35

- Leghe metalliche. . . . . . 37

- Metallocromia......... 42

- Monete, pesi e mis. ingl. . 43

- Problemi di geometria... 47

- Ricettario domestico . . . 49

- Ricettario industriale. . . . 49

Gibelli G. Idroterapia . . . . . 34

Giglioli E. H. Zoologia. ..... 57

Gioppi L. Crittogratia ...... 22

- Dizionario fotografico . . . 25

- Fotograffa industriale .. . 30

Giordani G. Proprietario di caso 48

Giorgetti S. Stenografia..... 52

Giorli E. Disegno industriale . 24

- Disegno e costruz. Nave. . 24

- Aritmetica e Geometsia . 13

- Meccanico (Il)........ . 41

- Meccanica (La) del macchinista di bordo......41

Gitt: V. Computisteria . . . . . 20

- Ragioneria.......... 48

Giudici 0 . Tessuti di lana e cot. 55

Gladstone W. E. Omero . . . . 44

Gnecchi F. Monete romane. . . 43

- Guida numismatica . . . . 34

Gobbi U. Assicuraz. generale 13

Goffi V. Disegnat. meccanico. 24

Gorini G. Colori e vernici ... 19

- Concia delle pelli ......20

- Conserve alimentari. . . . 20

- Metalli preziosi....... 42

- Olii. . . . . . . . . . . 44

- Piante industriali . . . . 45

- Pietre preziose... . . . 45

Gorra E. Lingue neo-latine . 39

- Morfologia italiana ... . . 43

Grawinkel, Elettrotecnica. . . . 27

Grassi F. Magnetismo $\mathrm{A}$ elett. 40

Grazzi-Soncini G. Vino (II) ...57
Griffini A. Coleotteri italiani. . 19

- Ittiologia italiana .... 36

- Lepidotteri italiani . . . . 37

- Imenotteri italiani. . . . . . 35

Grothe E. Filatura, tessitura. . 29

Grove G. Geografia.......31

Guaita L. Colori e la pittura 19

Guasti C. Imitazione di Cristo 35

Guelfi G. Vocabolario araldico $\mathbf{5 7}$

Guetta P. Il Canto ....... 16

Guyon B. Grammatica Slovena 34

Haeder H. Costr. macc. a vap. . 39

Hoepli U. Enciclopedia . . . . 27

Hooker I. Botanica......... 15

Hubert I. C. Antich. pubbl. rom. 12

Hugues L. Esercizi geografici . 27

- Cronologia scop. geogr. . . 22

Imitazione di Cristo. ....... 35

Imperato F. Attrezz. delle navi 14

Inama V. Antichità greche... 12

- Letteratura greca...... 38

- Grammatica greca. . . . . . 33

- Filologia classica . . . . . 29

- Florilegio poetico . . . . 29

- Esercizi greci. . . . . . . 28

Issel A Naturalista viaggiat.. 44

jacoangeli 0 . Triangol, topog.. 55

Jenkin F. Elettricità ......26

Jevons $W$. Stamley. Econ. polit. 26

- Logica. . . . . . . . 39

Jona E. Cavi telegr. sottomar. 17

Jones E. Calore (II) . . . . . 16

- Luce e suono... . . . . 39

Kiepert R. Atl. geogr. univers. 14

- Esercizi geografici. . . . . 27

Kopp W. Antich. priv. dei Rom. 12

La Leta B. M. Cosmografia. . . 22

- Enomonica.......... 33

Landi D. Dis. di proiez. ortog. 24

Landi S. Tipografia $\left(I^{\circ}\right)$ Guida 55

- $\left(\mathrm{II}^{\circ}\right)$ Compositore-tipogr. . 55

- Vccabolario tipografico . . 57

Lange 0 . Letteratura tedesca. 38

Lanzoni P. Geogr. comm. econ. 32

Larice R. Storia del commercio 20

Leoni B. Lavori in terra. . . 37

Lepetit R. Tintore ....... 55

Levi C. Fabbricati civ. di abitaz. 28

Levi c. Letterat. drammatica. 38

Levi I. Gramm. lingua ebraica. 33

Librandi V. Gramm. albanese. 33

Licciardelli G. Coniglicoltura . 20

Licò N. Protez. degli animali. 48

Lignarolo M. Doveri del macch. 26

- Macchinista navale ... 40

Lion A. Ingegneria legale . . 36

Lioy P. Ditteri italiani . . . . 25

Livi L. Antropometria...... 12 
Lockyer I. N. Astronomia. ... Lombardini A. Anat. pittorica. 12 Lombroso C. Grafologia. .... 33 Lomonaco A. Igiene della vista 34 Loria L. Macchinista e fuochis. 40 Loris. Diritto anmuinistrativo. 23 - Diritto civile ..... . . 23 Lovera R. Gramm. greca mod. . 83 - Grammiatica rumena . . . . 34 Luxardo 0. Merceologia. ....442 Mafílioli D. Diritti e dov. dei citt. 23 - Scritture d'affari......5 Maggi L. Protistologia ... . . 48 - Tecnica protistologica...54 Magrini E. Infortuni sul lavoro 36 - Case operaie. . . . . . . 17

hainardi G. Esattore...... 27 Majnoni R. Massaggio . . . . 41 Malacrida G. Materia medica 41 - Impiego ipodermico e la dusatura dei rimedi ..... 35

Malfatti B. Etnografia . . . . 28 28

Mancini P. La rachitide. .... 48

Manetti L. Caseificio....... 17

Mantovani G. Psicolog. fisiolog. 48

Marazza E. Stearineria .....552

- Saponi (Industria dei) . . 50

Parcel C. Lingue straniere . . 39

Marchi E. Maiale (II). . . . . . 40

Marcillac F. Letterat. francese 38 Marro A. Corr. elettr. alternate 21 Marzorati E. Codice perito mis. 18 Mastrigli L. Cantante . . . . 16 - Pianista.......... 46 Mattei C. Volapülk (Dizion.) . . 57 Hilazzocchi L. Calci e cementi. 16 - Cod. d. perito misuratore. 18 Mazzoccolo E. Legge comunale 37 Malani A. Architett. italiana . 12 - Decoraz. e industrie artist. 23

- Ornatista......... 45

- Pittura italiana. . . . . . 46

- Scultura italiana...... 51

Hielli B. L' Eritrea ........ . 27

Menozzi. Alimentaz. bestiame 11 Hercanti F. Animali parassiti . 12 Mezzanoiti C. Bonifiche..... 15 Mina G. Modellat. meccanico . 43 Minozzi A. Fosfati ....... 30 rinutti R. Letterat. tedesca . . 38 - Traduttore tedesco ..... 55 Molina E. Antologia sten ografica 12 Molina. Curatore dei fallimenti 22 Molina R. Esplodenti. . . . . . 28 molon \&. Ponologia .......47 Mondini. Produzione dei vini . 48 Montagna A. Fotosmaltogratia 30 Hontemartini L. Fisiol. veget. . 29 *areschi $N$. Antichità private dei Romani .......... 12 Morgana G. Gramm. olandese. 33 Morini U. Uffic. (Man. per l') . 56 Morselli E. Sociologia generale 52 Motta G. Telefono....... 54 Muffone \&. Fotografia ...... . 30 füller L. Metrica Greci e Rom. 42 mïller 0. Logaritmi . . . . . . 39

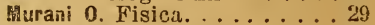
- Telegrafia senza fili. . . . 54 Murari R. Ritmica. . . . . . . 50 Iึusatti E. Jeggende popolari. 37 inuzio C. Medico pratico....41 - Malattie dei paesi caldi . . 40 Naccari G Astronoma nautica 14 Nallino A. Arabo parlato .... 12 Namias R. Fabbr. degli specchi 52 - Processi fotomeccanici... 47 Nazari 0. Dialetti italici .... 23 Negrin C. Paga giornaliera. . . 45 Henci T. Bachi da seta ..... 14 Niccoli V. Alimentaz. bestiame 11 - Cooperative rurali. .....21 - Costruzione ed economia dei fabbricati rurali .....28 - Prontuario dell'agricoltore. 10 Nicoleiti A Stenogaafia ..... 52 - Esercizi di stenografia... 52 Noseda E. Legislaz. Sanitaria 37 - Lavoro delle donne e fanc. 37 Nuyens A. Diz. italiano-oland. 25 Olivari G. Filonauta ...... 29 Olmo C. Dirito ecclesiastico. .23 Orlandi G. Celerimensura.... 17 Orsi P. Storia d'Italia..... . 53 Orsini E. Scacchi ........ 50 Ostwald-Bolis. Chimica analitica 17 Ottavi 0. Enologia ....... 27 - Viticoltura.......... 57 Ottino G. Bibliografia. ...... 15 Pagani C. Assicuraz. sulla vita 13 Paganini A. Letterat. francese 38 Paganini P, Fotogrammetria. . 30 Palombi A. Manuale postale. 47 Palumbo R. Omero... . . . . 44 Panizza F. Aritmetica razion. 13 - Aritmetica pratica,..... 13 - Esercizi di Aritmetica raz. 13 Pao'oni P. Disegno assonom. . 24 Pappalardo A. Spiritismo.... 52 - Telepatia......... 54 Parise P. Ortofrenia....... 45 Paroli E. Grammatica svedese 34 Pascal T. Tintura della seta . 55 Pascal E. Calcolo differenziale 16 - Calcolo inteurale. . . . . 16 - Calcolo delle variazioni . 16 
Pascal E. Esercizi di calcolo. 16 - Determinanti ......... 23

- Funzioni ellittiche .... 31

- Gruppi di trasformazioni . . 34

- Matematiche superiori . . .41

Pattacini G. Conciliatore . . . 20

Pavanello $F$ A. Verbi latini ...56

Pavia L. Grammatica tedesca. 34

- Grammatica inglese. . . . 33

- Grammatica spagnuola . . 34

Pavolini E. Buddismo ...... 15

Pedicino N. A. Botanica..... 15

Pedretti G. Automobilista (L'). 14

Pedrini. Casa dell' avvenire .. 17

Peglion v. Filossera ...... 29

Perassi T. G. Sintassi latina. . 51

Percossi R. Calligrafia. . . . . 16

Perdoni T. Idraulica . . . . . . 34

Petri L. Computisteria agraria 20

Petzholdt. Bibliotecario..... 15

Piazzoli E. Illuminaz. elettrica 35

Piccinelli F. Società Ind. p. az. 51

- Valori pubblici .......56

Piccinini P. Farmacoterapia . . 29

Piccoli D. V. Telefono..... 54

Pieraccini A. Assist. dei pazzi 13

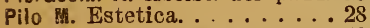

- Psicologia musicale.... 48

Pincherlo S. Algebra element. 11

- Algebra complementare . 10

- Esercizi di alogebra elem. . 11

- Esercizi di geometria. . . . 27

- Geometr. metr. e trigonometria ........... 32

- Geometria pura. ...... 32

Pinchetti P. Tessitore ..... . 54

Pini P. Epilessia . . . . . . 27

Pisani A. Mandolinista . . . . 41

- Chitarra ......... 18

Pizzini L. Disinfezione . . . . 24

- Microbiologia . . . . . . 42

Pizzi I. Letteratura persiana 38

- Islamismo ......... . 36

- Letteratura araba .... . . 37

Piebani B. Arte della memoria 13

Polacco L. Tav. Div. Comm. . 54

Poloni G. Magnet. ed elettricita 40

Pompilio. Panificazione .....44

Porro F. Spettroscopio . . . . 52

- Gravitazione. . . . . . 34

Portigliotti G. Psicoterapia . . 48

Pozzi G. Regolo calcolatore . . 49

Prat G. Grammatica francese. 33 - Esercizi di traduzione . . .28

Prato G. Cognac. . . . . . . 19

- Vini bianchi........ 57

Proctor R. A. Spettroscopio . . 52

Prout E. Strumentazione. . . . 53
Pucci A. Frutta minori . . . . 31

- I'iante e fiori....... 46

Rabieno A. Mezzeria. . . . . 42

- Ipoteche (Manuale per le) 36

Racioppi F. Ordinamento degli

Stati liberi d'Europa.... 45

- Idem, fuori d'Europa . . . . 45

Raina M. Logaritmi . . . . . . 39

Ramorino F. Letterat. romana. 38

- Mitologia (Dizionario di) . 43

Rebuschini E. Mal. del sangue. 40

- Organoterapia. ...... 45

- Sieroterapia... . . . . . 51

Regazzoni J. Paleoetnologia . . 45

Repossi A. Igiene scolastica. . 34

Restori A Letterat. provenzale 38 - Letteratura catalana.... 37

Revel A. Letteratura ebraica . 38

Ricci A. Marmista . . . . . 41

Ricci E. Chinica ....... . 17

Ricci S. Epigrafia latina... . 27

- Archeologia. Arte greca. . 12

- Arte etr.e rom. 12

Picci v."Strumentazione . . . 53

Righetti $\mathrm{E}$ Asfalto ........ 13

Rivelii A. Stereometria . . . 52

Roda F Ili. Floricoltura . . . . 29

Rodari D. Sintassi francese...51

- Esercizi relat. a sintassi : 51

Romanelli-r.

Ronchetti G. Pittura per dilett. 46

- Grammatica d. disegno . . 24

Roscoe H. E. Chimica ...... 17

Rossetto V. Arte militare. . . 53

- Avarie e sinistri marittimi 14

Rossi A. Liquorista. . . . . . . 39

- Profumiere.......... 48

Rossi G. Costruttore navale . 22

Rossotti H. A. Formul. di matem. 30

Rota G. Ragioneria delle cooperative di consumo.....49

- Contabilità (v. Beneficenza) 15

Ruata G. Ufficiale sanitario . .56

Saccheri P.G.L'Euclide emendato 28

Sacchetti G. Tecnologia e terminologia monetaria. . . . 54 Salvatore A. Infort. sul lavoro 37 Sanarelli. Igiene del lavoro.. 34 Sansoni F. Cristallografia. . . 22 Santi B. Diz. dei Comuni ital. 25 Santilli. Selvicoltura...... 51 Sanvisenti B. Letteratura spag. 38 Sartori G. Latte, burro e cacio 36 - Caseificio. . . . . . . . 17

Sartori L. Carta (Industr. della) 17

Sassi L. Carte fotografiche . 17

- Ricettario fotografico . . 5 50

- Fotocromatografia. . . . . 30 
Sassi L. Proiezioni (Le). . . . 48 Savorgnan. Coltiv.d. piante tess. 46 Scarpis U. Teoria dei numeri. 54 Scartazzini G. A. Dantologia.. 23 Schenck E. Resist. travi metal. 43 Schiaparelli G. V. L'astronomia

n. antico testamento. .... 14 Schiavenato A. Diz. stenogr. . 25 Scolári C Dizionario alpino.. 25 Secco-Suardo. Ristau. dipinti . 50 Seghieri A. Scacchi. ....... 50 Sella A. Fisica cristallografica 29 Serina I. Testamenti ...... 55 Sernagiotto R. Enol domestica 27 Sessa G. Dottrina vopolare . . 26 Setti A. Man. del Giurato . . . 33 Severl A. Monogrammı.....43 Siber-millot C. Molini (Ind. dei) 43 Solazzi E. Letteratura inglese. 38 Soldani G. Agrnnom. moderna 10 Solerio G. P. Rivoluz. francese 50 Soli G. Didattica

Spagnotti P. Verbi greci . . . 56 Spataro D. Fognat. cittadina . 30 Stecchi R. Chirurgia operat. . 18 Stoppani A. Geografia fisica.

- Geologia.

- Prealpi bergamasche.

Stoppato A. Diritto nenale . . 24

Stoppato L. Fonologia italiana 30 Strafforello $\mathrm{G}$. Alimentazione . 11

- Errori e pregiudizi ... . . 27

- Letteratura americana... 37

Straticd A. Letterat. albanese. 37

Streker. Elettrotecnica ..... 27

Strucchi A. Cantiniere. . . . . 16

- Enologia

- Viticoltura

Supino R. Chimica clinica . . 17

Tabanelli $N$. Codice del teatro 19

Taccani A. Zucchero (Fabbr. d.) 57

Tacchini A. Metrologia ..... 42

Tamaro D. Frutticoltura ... 31

- Gelsicoltura

- Orticoltura.

- Uve da tavola.

Tami F Nantion stimat......

Tampelini G. Zuotecnia ....57

Taramelli A. Prealpi bergamas. 47 Teloni B. Letteratura assira. . 38 Thompson E. M. Paleografia . 45 Tioli L. Acque minerali e cure 10 Tognini A. Anatomia vegetale. 12 Tolesani D. Enimmistica . . . 27 Tommasi M. R. Convers. volapük 57 Toniazzo G. St. ant. (La Grecia) 53 Tonta I. Raggi Röntgen ., . . 50 Tonzig C. Ufficiale sanitario. . 5c,
Tozer H. F. Geografia classica. 32 Trabaiza C. Insegn. dell'italiano 36 Trambusti A. Igiene del lavoro 47 Trasporti e tariffe ferroviarie .. 55 Trevisani G. Pollicoltura ....47 Tribolati F. Araldica (Gramm.) 12 Tricon E. Medicat. antisettica 41 Trivero C. Classific. d. scienze 18 Ulivi $\mathrm{P}$. Industria frigorifera. 35 Untersteiner A. Storia musica. 53 Untersteiner L. Uccelli canori. 56 Vacchelli G. Calcestruzzo .... 16 Valenti A. Aromatici e nervini 13 Valentini $N$. Chimica legale . 17 Valletti F. Ginnast. femminile. 32 - Ginnastica (Storia della). . 32 Valmaggi L. Gramm. latina. .. 33 Vanbianchi C. Autografi. .... 14 Vecchio A. Cane (II) ....... 16 Vender $\forall$.Acido solforico, ecc. 10 Venturoli G. Concia pelli . ... 20 - Conserve alimentari ... . 20 Vidari E. Diritto commerciale. 23 - Mandato commerciale .. 40 Vidari G. Etica. .......... 28 Villani F. Prodotti della distillazione del legno......24 Vinassa P. Paleontologia. . . 45 Virgilii $F$. Cooperazione.....21 - Economia niatematica... . - Statistica......... 52 Viterbo E. Grammatica e dizion. Galla (Oromonica)... 33 Vitta C Ginstizia amministr. . 33 Vivanti G. Funzioni analitiche 31 - Comp. matematica ..... 41 Yoigt W. Fisica cristallograf. . ig Voinovich. Grammatica russa. 34 - Vocabolario russo ..... 57 Volpini C. Cavallo........ 17 - Proverbi sul cavalio ... . 48 Webber E. Macchine a vapore 39 - Dizionario tecnico italianotedesco-francese-inglese. . . 26 Werth F. Galvanizzazione ... 31 Will. Tav. analit. (v. Chimico) 17 wittgens A. Antic. pubhl. rom. 12 W. If R. Malattie crittogam... 40 Zambelii A. Manuale di conversaz. italiano-volapük .. 57

Zambler A. Medicat. antisett. . 41 Zampini G. Bibbia (Man. deila) 15 - Imitazione di cristo. . . . 35 Zịgány-Ârpád. Lett. ungherese. 38 zoppetti v. Arte mineraria. 13-42 - Siderurgia ........... 51 Zubiani $A$. Tisici e sanatorii 55 Zucca A. Acrohatica e atletica 10 
5 



